\title{
The Bulk Viscosity of Suspensions
}

\author{
Thesis by \\ Manuj Swaroop \\ In Partial Fulfillment of the Requirements \\ for the Degree of \\ Doctor of Philosophy
}

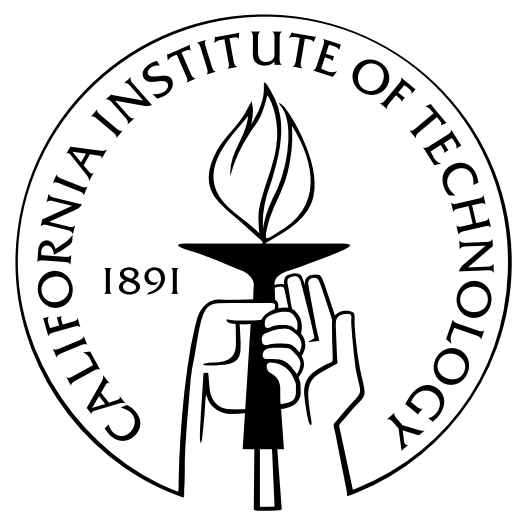

California Institute of Technology

Pasadena, California

2010

(Defended April 15, 2010) 
(C) 2010

Manuj Swaroop

All Rights Reserved 


\section{Acknowledgements}

The years I have spent at Caltech have been some of the best of my life. It has been a very stimulating, friendly and enjoyable experience and I am very grateful to the Institute and all the people here who have enriched my life.

Most of all I am very thankful to Prof. John F. Brady for agreeing to be my advisor. John has always brought out the best in me and he knows exactly how to help his students make the best choices in their career. He has always encouraged me and trusted me in times of both elation and despair, which enabled me to put the best effort I could in my research. There is nothing more I could have expected from my advisor.

I would like to take this opportunity to thank the members of my thesis committee, Prof. John H. Seinfeld, Prof. Zhen-Gang Wang, Prof. Melany L. Hunt, and of course my advisor, Prof. John F. Brady for taking interest in my research and enabling me to reach the conclusion of my work at Caltech.

I have been fortunate to work in a research group with some of the smartest, friendliest and most helpful people I have met: Aditya Khair, Josh Black, Ubaldo Cordova, James Swan, Andy Downard, Roseanna Zia, Ashley Smart and Nick Hoh. Special thanks go to Rozeanna N. Zia for stimulating conversations and being a good friend. I would also like to thank James Swan for providing me with some of his excellent code upon which I was able to build my simulation work, and Thanh Ngoc Phung who wrote the older code that I have used in my research.

I made some great and lasting friends at Caltech and my life is much richer because of them: Sawyer Fuller, David Gleason, Erik Schomburg, Gerardo Cruz, Shaunak Sen, Nachiket Kapre, Gunjan Sukul, Pratyush Tiwary, Pinkesh Patel, Varun Bhalerao, Teja Sukhavasi; the list is too long and so I offer my sincerest apologies those whom I did not mention.

I would like to thank the Chemical Engineering staff for always being prompt and available to 
help the students, especially Kathy Bubash, Laura Lutz King and Suresh Guptha, for taking care of all the details and making my life so much easier. The staff at the Graduate Office, especially Natalie Gilmore, and Jim Endrizzi and Laura Flower Kim at the ISP have been extremely helpful in making my experience at Caltech a memorable one and for that I am very thankful to them.

Being able to go to a prestigious school like Caltech is a great achievement for me and it would not have been possible without the love, support and dedication of my parents. They always gave me the best of things and the best advice and always put my happiness ahead of their aspirations. I will always aspire to keep them happy and try to make their life as fulfilling as they wish.

I saved the last but the biggest thanks for the most loving and caring person I have ever met: my wonderful wife Neha. As I write this we are close to being married for two years and these years that I have spent with her have been amazing to say the least. I am sure that years from now we will both remember our time at Caltech as the most fun years of our time together. 


\section{Abstract}

Particles suspended in a fluid are known to undergo variations in the local concentration in many flow situations; essentially a compression or expansion of the particle phase. The modeling of this behavior on a macroscopic scale requires knowledge of the effective bulk viscosity of the suspension, which has not been studied before. The bulk viscosity of a pure compressible fluid is defined as the constant of proportionality that relates the difference between the mechanical pressure and the thermodynamic pressure to the rate of compression. The bulk viscosity of a suspension is defined analogous to that for a pure fluid as the constant of proportionality relating the deviation of the trace of the macroscopic stress from its equilibrium value to the average rate of compression. The compression flow drives the suspension microstructure out of equilibrium and the thermal motion of the particles tries to restore equilibrium. The Peclet number $(\mathrm{Pe})$, defined as the expansion rate made dimensionless with the Brownian time-scale, governs the departure of the microstructure from equilibrium. The microstructural forcing in compression is monopolar for small $\mathrm{Pe}$ resulting in a significantly slower spatial and temporal response of the microstructure compared to shearing or diffusive motion.

We have determined the effective suspension bulk viscosity for all concentrations and all rates of compression, accounting for the full thermodynamic and hydrodynamic interactions that particles experience at the micro-scale. Current simulation techniques were enhanced to enable the dynamic simulation of compression flows in a suspension. A 'compression thinning' of the suspension is observed at small rates of compression and there is some 'compression thickening' at large compression rates. The bulk viscosity diverges as the volume fraction nears maximum packing and is in fact larger than the shear viscosity. Existing models for multiphase flows must therefore include the bulk viscosity term to properly simulate variations in particle concentration.

An understanding of bulk viscosity effects in suspensions will enable the modeling of certain 
aggregation and separation behavior and lead to more accurate models for multiphase flows where there are variations in the particle concentration, such as filtration or fluidization. 


\section{Contents}

Acknowledgements

$\begin{array}{lll}\text { Abstract } & \text { v }\end{array}$

List of Figures $\quad$ xviii

List of Tables $\quad$ xx

1 Introduction 1

2 Dilute Theory 9

2.1 Measurement of the bulk viscosity . . . . . . . . . . . . . . . . 11

2.2 Bulk viscosity of a suspension $\ldots \ldots \ldots \ldots \ldots \ldots$

2.3 The $O\left(\phi^{2}\right)$ correction to bulk viscosity $\ldots \ldots \ldots \ldots \ldots \ldots$

2.3.1 Pair-evolution equation . . . . . . . . . . . . . 17

2.3.2 Hydrodynamic interactions . . . . . . . . . . . . . . . . 23

2.3.3 Expressions for the bulk viscosity . . . . . . . . . . . . . . 24

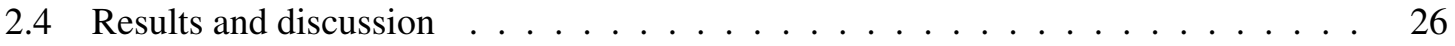

2.4.1 Numerical solution of the perturbed microstructure . . . . . . . . 26

2.4.2 The $O\left(\phi_{b}^{2}\right)$ bulk viscosity coefficients . . . . . . . . . . . 28

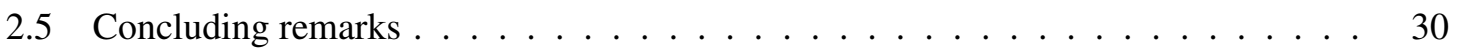

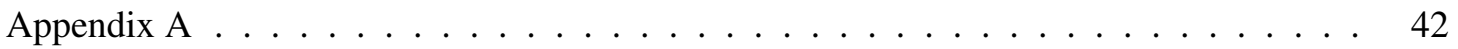

3 The Bulk-Viscoelasticity of Suspensions

3.1 Introduction . . . . . . . . . . . . . . . . . . . . 49

3.2 Bulk viscosity of a suspension $\ldots \ldots \ldots \ldots \ldots$ 
3.3 Microstructure and bulk viscosity: No hydrodynamics . . . . . . . . . . . . . 55

3.4 Temporal response $\ldots \ldots \ldots \ldots \ldots \ldots \ldots$

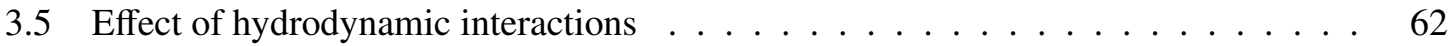

3.6 Numerical results . . . . . . . . . . . . . . . . . . . . . . . . . . . . 69

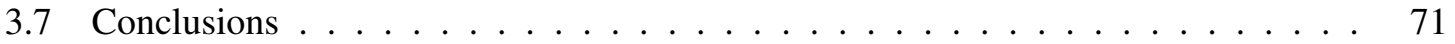

4 Stokesian Dynamics Simulations for Compressible Flows of Suspensions 78

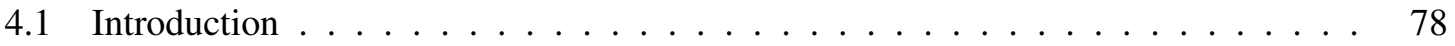

4.2 Suspension stress $\ldots \ldots \ldots \ldots \ldots \ldots \ldots$

4.3 Stokesian Dynamics . . . . . . . . . . . . . . . . . . . . 82

4.3.1 Review of the existing method . . . . . . . . . . . . . 83

4.3.2 Linear compressible flow . . . . . . . . . . . . . . . 86

4.3.3 The Mobility matrix with expansion flow . . . . . . . . . . . . . 88

4.3 .4 Brownian motion . . . . . . . . . . . . . . . . . . . . . 90

4.4 Accelerated Stokesian Dynamics . . . . . . . . . . . . . . . . . . . . . . . . 91

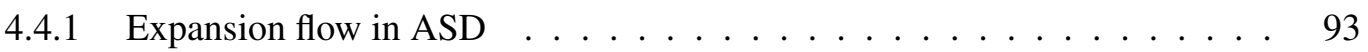

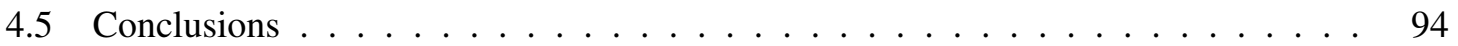

Appendix B . . . . . . . . . . . . . . . . . . . 95

5 Equilibrium Properties via Simulation $\quad 98$

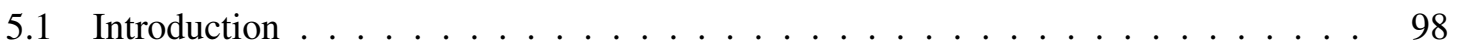

5.2 Suspension stress and the bulk viscosity $\ldots \ldots \ldots \ldots \ldots$

5.3 Brownian Dynamics simulations $\ldots \ldots \ldots \ldots \ldots 1 \ldots \ldots \ldots$

5.3 .1 Simulation results $\ldots \ldots \ldots \ldots \ldots$

5.3.2 Scaling with volume fraction . . . . . . . . . . . . . . . 106

5.3 .3 Temporal scaling . . . . . . . . . . . . . . . . . . . 108

5.4 Effect of hydrodynamic interactions f . . . . . . . . . . . . . 112

5.4 .1 Scaling with volume fraction . . . . . . . . . . . . . 113

5.4 .2 Temporal scaling . . . . . . . . . . . . . . 116

5.4 .3 Simulation results . . . . . . . . . . . . . . . 120

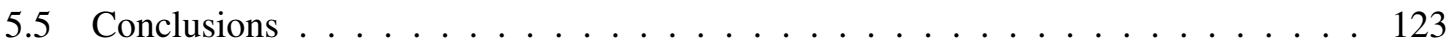


6 Simulation of Compression Flows in Suspensions 163

6.1 Introduction . . . . . . . . . . . . . . . . . . . 163

6.2 Simulation procedure . . . . . . . . . . . . . . . . . . 164

6.3 Microstructure in compression . . . . . . . . . . . . . . . . 166

6.4 Brownian Dynamics $\ldots \ldots \ldots \ldots \ldots$

6.4.1 Results and scaling . . . . . . . . . . . . . . . . . 171

6.5 Accelerated Stokesian Dynamics . . . . . . . . . . . . . . . . . . . . . 174

6.5.1 Results and scaling . . . . . . . . . . . . . . . . . 176

6.6 Effect of shear on the hydrodynamic bulk viscosity $\ldots \ldots \ldots \ldots$

6.7 Conclusions . . . . . . . . . . . . . . . . . . . . . . . . 179

7 Concluding Remarks $\quad 213$

$\begin{array}{ll}\text { Bibliography } & 217\end{array}$ 


\section{List of Figures}

2.1 The functions $T_{11}^{Q}$ (solid line) and $T_{12}^{Q}$ (dashed line) for equal-sized spheres vs the

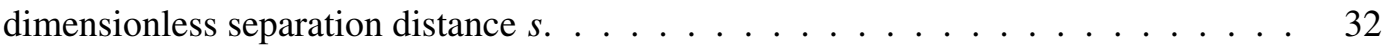

2.2 Microstructural perturbation function $f(r)$ for $b / a=1.00001$ (dashed line) and $b / a=$ 1000 (solid line) for $\left|P e_{b}\right| \ll 1$. Both curves behave as $1 / r$ for large $r$ like the linear response solution. For other values of $b / a$ the perturbation $f$ is intermediate between

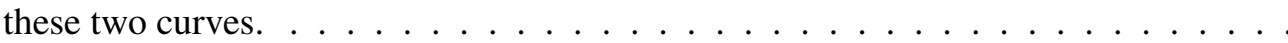

2.3 Microstructural perturbation function $f(r)$ close to the particle's surface for full hydrodynamics, $b / a=1.00001$ (dashed line) and for no hydrodynamics, $b / a=1000$ (solid line) and $P e_{b}=-10^{3}$. Both curves decay much faster than for small $P e_{b}$. For other values of $b / a$ the perturbation $f$ is intermediate between these two curves. . . .

2.4 Microstructural perturbation function $f(r)$ at contact $(r=2)$ vs $P e_{b}$ for varying levels of hydrodynamic interactions. All the curves have the same behavior and exhibit a shear-thinning like behavior. The curve for no hydrodynamics corresponding to (2.29) is shown by a solid line. . . . . . . . . . . . . . . . . . . . . .

2.5 Brownian part of the $O\left(\phi_{b}^{2}\right)$ two-particle contribution to the bulk viscosity $\kappa^{B} / \eta$ vs $\hat{b}-1$, for different negative values of $P e_{b} \ldots \ldots \ldots \ldots$

2.6 Interparticle force part of the $O\left(\phi_{b}^{2}\right)$ two-particle contribution to the bulk viscosity $\kappa^{P} / \eta$ vs $\hat{b}-1$, for different negative values of $P e_{b} \ldots \ldots \ldots \ldots$

2.7 Rate-of-strain part of the $O\left(\phi_{b}^{2}\right)$ two-particle contribution to the bulk viscosity $\kappa^{E} / \eta$ vs $\hat{b}-1$, for different negative values of $P e_{b} . \ldots \ldots \ldots$ 
2.8 The total $O\left(\phi_{b}^{2}\right)$ two-particle contributions to the bulk viscosity $\left(\kappa^{B}+\kappa^{P}+\kappa^{E}\right) / \eta$ vs $P e_{b}$, for varying levels of hydrodynamic interaction. Shear thickening is observed as $P e_{b} \rightarrow-\infty$. As $\hat{b} \rightarrow \infty$ hydrodynamic interactions between particles decrease and

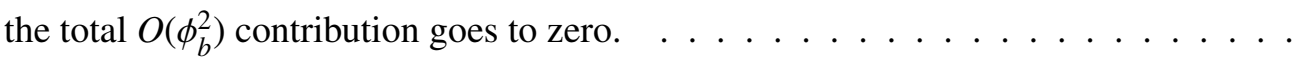

2.9 Bulk viscosity for hard spheres of radius $b$ with no hydrodynamic interactions. Only the interparticle force comprising the hard sphere potential contributes to the bulk stress. 40

2.10 The $O\left(\phi^{2}\right)$ two-particle contributions to the bulk viscosity for small $P e_{b}$ (linear response regime) : Brownian $\kappa^{B} / \eta$ (dashed line), interparticle-force $\kappa^{P} / \eta$ (dot-dashed line), rate-of-strain $\kappa^{E} / \eta$ (dotted line) and total $\left(\kappa^{B}+\kappa^{P}+\kappa^{E}\right) / \eta$ (solid line). Shown here are the coefficients based on the actual particle volume fraction $\phi . . . . .$. .

3.1 Real reduced functions for the bulk viscosity (solid curve) and shear viscosity (dashed curve) of hard spheres with no hydrodynamic interactions. . . . . . . . . . . . . 72

3.2 Imaginary reduced functions for the bulk viscosity (solid curve) and shear viscosity (dashed curve) of hard spheres with no hydrodynamic interactions. . . . . . . . . 73

3.3 The scaled Pressure autocorrelation function $\overline{C_{N H}^{P}}$ (solid curve) for hard spheres with no hydrodynamic interactions. The corresponding shear-stress autocorrelation function $\overline{C_{N H}^{S}}$ (dashed curve) is also shown for comparison. . . . . . . . . . . . .

3.4 Real part of the reduced bulk viscosity function $\kappa_{r}^{\prime}(\alpha)$ as a function of the nondimensional frequency $\alpha=\omega b^{2} / 2 D$ at varying levels of hydrodynamic interactions from nearly full $\left(\hat{b}-1=10^{-6}\right)$ to no hydrodynamics $\left(\hat{b} \simeq 10^{5}\right)$. The steady expansion limit of the bulk viscosity without hydrodynamics is $\kappa^{P}(0)=12 \eta \phi_{b}^{2}$ and with full hydrodynamics $\kappa^{B}(0)=5.247 \eta \phi_{b}^{2} \ldots \ldots \ldots \ldots \ldots \ldots \ldots \ldots \ldots \ldots \ldots \ldots$

3.5 Imaginary part of the reduced bulk viscosity function $\kappa_{r}^{\prime \prime}(\alpha)$ as a function of the nondimensional frequency $\alpha=\omega b^{2} / 2 D$ at varying levels of hydrodynamic interactions from nearly full $\left(\hat{b}-1=10^{-6}\right)$ to no hydrodynamics $\left(\hat{b} \simeq 10^{5}\right)$.

3.6 The elastic bulk modulus $K^{\prime}(\alpha)$ as a function of the nondimensional frequency $\alpha=$ $\omega b^{2} / 2 D$ at varying levels of hydrodynamic interactions from nearly full $\left(\hat{b}-1=10^{-6}\right)$ to no hydrodynamics $\left(\hat{b} \simeq 10^{5}\right)$. The high-frequency limit with full hydrodynamics is $K^{\prime}(\infty) \simeq 0.56\left(k T / a^{3}\right) \phi_{b}^{2} \ldots \ldots \ldots \ldots \ldots \ldots \ldots$ 
5.1 The equilibrium osmotic pressure $\Pi_{0}$ determined from Brownian Dynamics (open circles) and Accelerated Stokesian Dynamics — near field simulations (black squares) nondimensionalized with $n k T$, as a function of the volume fraction $\phi$. The data was averaged over all the configurations occurring in the equilibrium simulations for each $\phi$ for each of the simulation methods ( $\sim 10^{6}$ realizations). The theoretical value given by $\overline{\Pi_{0}}=1+4 \phi g_{0}(2 ; \phi)$ is also shown with $g_{0}(2 ; \phi)$ data from Rintoul and Torquato [1996] for the $\phi>0.55$. The pressure data from Speedy [1994] is also shown for the metastable and glassy region $\ldots \ldots \ldots \ldots \ldots \ldots$

5.2 Pressure autocorrelation function from Brownian Dynamics with $N=100$ particles as a function of the diffusive time $\ldots \ldots \ldots \ldots \ldots \ldots$

5.3 Pressure autocorrelation function from Brownian Dynamics with $N=1000$ particles as a function of the diffusive time. . . . . . . . . . . . . .

5.4 Comparison of the scaled pressure autocorrelation function $\overline{C_{N H}^{P}}=C_{N H}^{P} N /\left(\phi g_{0}(2 ; \phi)\right)$ (5.22) from Brownian Dynamics with (a) $N=1000$ and (b) $N=100$ particles as a function of the diffusive time for intermediate volume fractions. The plots illustrate the faster decay of pressure fluctuations for $N=100$ due to finite-cell-size effects. For $N=1000$ the data is aligned very closely with the analytical curve. . . . . . . 128

5.5 Comparison of the scaled pressure autocorrelation function $\overline{C_{N H}^{P}}=C_{N H}^{P} N /\left(\phi g_{0}(2 ; \phi)\right)$ (5.22) from Brownian Dynamics with (a) $N=1000$ and (b) $N=100$ particles as a function of the diffusive time for high volume fractions. In both cases the time-scale of decay increases with increasing volume fraction for $\phi \geq 0.4$. The data for $\phi=0.05$ is also shown to demonstrate that for low $\phi$ (largest cell size simulated) the simulation data matches perfectly with the predicted theoretical curve. . . . . . . . . . . . 129

5.6 Stress relaxation time from data between $0<\tau \leq 0.7$ for the pressure autocorrelation function from BD simulations with $N=1000 \ldots \ldots \ldots \ldots$

5.7 Stress relaxation time from the data with fitted long-time tails between $0<\tau \leq 200$ for the pressure autocorrelation function from BD simulations with $N=1000 . \quad \ldots \quad$. 131 
5.8 The scaled Pressure autocorrelation function from Brownian Dynamics $\overline{C_{N H}^{P}}=C_{N H}^{P} N /\left(\phi g_{0}(2 ; \phi)\right)$ (5.22) with $N=1000$ particles with fitted long-time tails for all volume fractions simulated, as a function of the diffusive time. In plot (a) time is scaled with the bare diffusivity of he particles and in (b) with the characteristic diffusivity $\hat{D}(\phi)$. The latter scaling collapses all the long-time tails onto the predicted theoretical curve. . . . . . 132

5.9 The Green-Kubo bulk viscosity $\overline{\kappa^{B}}$ from equilibrium Brownian Dynamics simulations (solid diamonds), the MD simulation results of Sigurgeirsson and Heyes [2003] for the bulk viscosity of hard sphere fluids $(+$ and $*)$, and the scaled theoretical curve (solid line) $\ldots \ldots \ldots \ldots \ldots \ldots \ldots \ldots$

5.10 Shear stress autocorrelation function from Brownian Dynamics with $N=1000$ particles as a function of the diffusive time. . . . . . . . . . . . . . . . . . . . . 134

5.11 The scaled Shear stress autocorrelation function $\overline{C_{N H}^{S}}=C_{N H}^{S} N /\left(\phi g_{0}(2 ; \phi)\right)$ from Brownian Dynamics with $N=1000$ particles with fitted long-time tails in (b) for all volume fractions simulated, as a function of the diffusive time. In plot (a) time is scaled with the bare diffusivity of he particles and in (b) with the characteristic diffusivity $\hat{D}(\phi)$. The latter scaling collapses all the long-time tails onto the predicted theoretical curve. . . . . . . . . . . . . . . . . . . . . . . . 135

5.12 The zero-shear viscosity $\eta^{B}$ from equilibrium Brownian Dynamics simulations (solid diamonds), the simulation results of Foss and Brady [2000] for $\eta^{B}$ (open circles), and the scaled theoretical curve (solid line). The simulation data was obtained from equilibrium simulations with a total of $\sim 2 \times 10^{7}$ time-steps for each $\phi . \ldots 136$

5.13 Pressure autocorrelation function from Stokesian Dynamics simulations with $N=27$ particles as a function of the diffusive time. For $\phi>0.45$ the decay is exponential as the simulation cell size becomes small enough that the periodic boundary conditions have the effect of diffusion in a finite box. . . . . . . . . . . . . . . . . . 137 
5.14 Pressure autocorrelation function from Accelerated Stokesian Dynamics — near field simulations with $N=1000$ particles as a function of the diffusive time. For $\phi>0.52$, $C_{H}^{P}$ decays much more slowly because the particles are in the glassy regime. At long times the finite size of the simulation cell causes the pressure autocorrelation to decay exponentially as the periodic boundary conditions simulate diffusion of the particles

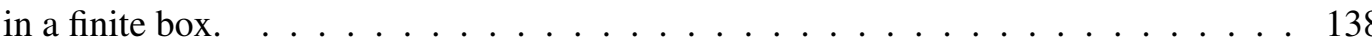

5.15 Scaled pressure autocorrelation function from ASDB-nf simulations $\overline{C_{H}^{P}}=C_{H}^{P} N D_{0}^{S}(\phi) / \phi g_{0}(2 ; \phi)$ with $N=1000$ particles as a function of the diffusive time, for (a) $0.05 \leq \phi \leq 0.35$ and (b) $0.35 \leq \phi \leq 0.52$. For the lower volume fractions the rate of decay decreases as $\phi$ increases while for $\phi \geq 0.35$ the rate of decay increases with $\phi$. The analytical curve for no hydrodynamics is shown by the black curve and has the same long-time behavior as the simulation data. Also shown is the zero-time limit from scaled dilute

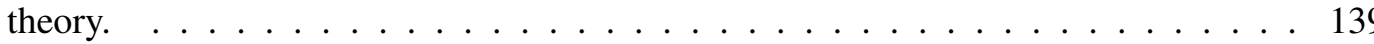

5.16 Comparison of the scaled pressure autocorrelation function from ASDB-nf simulations $\overline{C_{H}^{P}}=C_{H}^{P} N D_{0}^{s}(\phi) / \phi g_{0}(2 ; \phi)$ with the same cell size given by (a) $L=20.31 b$ and (b) $L=34.73 b$ but for different volume fractions. The decrease in the rate of decay with increasing $\phi$ for $\phi<0.35$ is evident in both plots, indicating that it is not a finite-size effect. . . . . . . . . . . . . . . . . . . . . . . . . . 140

5.17 Stress relaxation time from data between $0<\tau \leq 1$ for the pressure autocorrelation function from ASDB-nf simulations with $N=1000 \ldots \ldots \ldots \ldots \ldots$

5.18 Stress relaxation time from data between $0<\tau \leq 200$ with fitted long-time tails for the pressure autocorrelation function from ASDB-nf simulations with $N=1000$. . . 142

5.19 Scaled Pressure autocorrelation function from ASDB-nf simulations $\overline{C_{H}^{P}}=C_{H}^{P} N D_{0}^{s}(\phi) / \phi g_{0}(2 ; \phi)$ with $N=1000$ particles for all $\phi$ simulated, as a function of (a) the diffusive time and (b) diffusive time scaled with the stress relaxation time-scale. The long-time tails were obtained by fitting the analytical curve for no hydrodynamics (also shown) with the corresponding temporal scaling. . . . . . . . . . . . . . . . . . . . . 143

5.20 The Green-Kubo bulk viscosity $\overline{\kappa^{B}}$ as a function of volume fraction from equilibrium ASDB-nf simulations (solid squares) with $N=1000$, SD simulations (shaded circles) with $N=27$, and the scaled dilute theory (solid line). 
5.21 The high-frequency bulk modulus as a function of volume fraction from equilibrium ASDB-nf simulations (solid squares) with $N=1000$, SD simulations (shaded circles) with $N=27$, and the scaled dilute theory (solid line). . . . . . . . . . . . 145

5.22 Shear stress autocorrelation function from Accelerated Stokesian Dynamics - near field simulations with $N=1000$ particles as a function of the diffusive time. For $\phi>0.55$ the system is in a glassy state so the shear stress autocorrelation decays much more slowly. . . . . . . . . . . . . . . . . . . . . . . . 146

5.23 The scaled shear stress autocorrelation function from ASDB-nf simulations with $N=1000$ particles as a function of the diffusive time. The scaling in (a) is with the short-time self-diffusivity $\overline{C_{H}^{S}}=C_{H}^{S} N D_{0}^{S}(\phi) /\left(\phi g_{0}(2 ; \phi)\right)$, and in (b) with the highfrequency shear viscosity $\overline{C_{H}^{S}}=C_{H}^{S} N /\left(\phi g_{0}(2 ; \phi) \eta_{\infty}^{\prime}(\phi)\right)$. The analytical curve for no hydrodynamic interactions is also shown and has the same long-time behavior as the simulation data. $\ldots \ldots \ldots \ldots \ldots \ldots \ldots \ldots$

5.24 The scaled shear stress autocorrelation function from ASDB-nf simulations with $N=$ 1000 as a function of (a) the diffusive time, and (b) the diffusive time scaled with the stress relaxation time scale. The shear stress autocorrelation is scaled with the high-frequency shear viscosity $\overline{C_{H}^{S}}=C_{H}^{S} N /\left(\phi g_{0}(2 ; \phi) \eta_{\infty}^{\prime}(\phi)\right)$. The long-time tails are fitted with the analytical curve with no hydrodynamic interactions. . . . . . . . 148

5.25 The Green-Kubo shear viscosity $\overline{\eta^{B}}$ as a function of volume fraction from equilibrium ASDB-nf simulations (solid squares) with $N=1000$, Brownian Dynamics simulations (shaded diamonds) with $N=1000$, and the scaled dilute theory (solid line). Also shown are the simulation results of Foss and Brady [1999] (empty circles), experimental results of Segrè et al. [1995], and experimental results of Cheng et al.

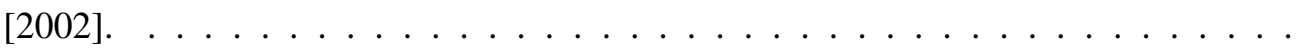

5.26 The high-frequency bulk viscosity due to interactions between particles as a function of volume fraction from equilibrium ASDB-nf simulations with $N=1000$, and the scaled dilute theory $($ solid line) $\ldots \ldots \ldots$. . . . . . . . . . . . . . . 150 
5.27 The total contribution to the bulk viscosity due to interactions between particles as a function of volume fraction from equilibrium ASDB-nf simulations with $N=1000$. Shown here is the direct hydrodynamic contribution (solid squares), the Green-Kubo Brownian contribution (solid squares), and their sum (shaded circles). . . . . . . . .

6.1 Planar plots of the pair distribution function $g(r)$ obtained from Brownian Dynamics simulations for $\phi=0.2$ and $\phi=0.45$ at compression rates of $P e=-1$ and $P e=-10$. Lighter regions indicate accumulation of particles. . . . . . . . . .

6.2 Radial pair distribution in compression for $\phi=0.2$ obtained from Brownian Dynamics with $N=1000$, for rates $P e=-1$ and $-10 \ldots \ldots \ldots \ldots \ldots$

6.3 Radial pair distribution in compression for $\phi=0.2$ obtained from Accelerated Stokesian Dynamics with $N=100$, for rates $P e=-1$ and $-10 \ldots \ldots \ldots \ldots$

6.4 Histogram of scaled particle hydrodynamic pressure moments $\left\langle S^{H}\right\rangle / \mathrm{Pe}$ obtained from Accelerated Stokesian Dynamics with $N=100,200$ distinct runs, for rates $P e=-1$

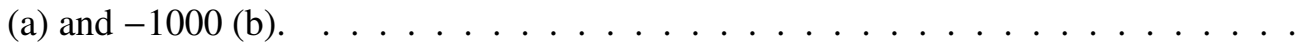

6.5 Two examples of particles with high hydrodynamic pressure moment $\left.\left(\left\langle S^{H}\right\rangle / \mathrm{Pe}\right\rangle 10\right)$ forming clusters distributed randomly in space at high rate $(P e=-1000)$, obtained from Accelerated Stokesian Dynamics with $N=100$. . . . . . . . . . . .

6.6 Nondimensional interparticle-force contribution to the pressure in compression vs Péclet number, from Brownian Dynamics with $N=1000$ for $\phi=0.1$ to $\phi=0.55$. The open circles denote the equilibrium particle contribution to the osmotic pressure. 186

6.7 The excess interparticle-force contribution to the pressure in compression normalized with $\phi g_{0}(2 ; \phi)$ vs Péclet number, from Brownian Dynamics with $N=1000$ for $\phi=$

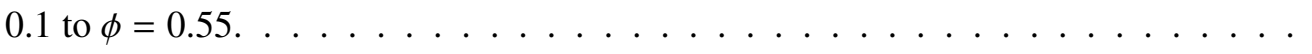

6.8 The nondimensional hard sphere bulk viscosity $\overline{\kappa^{P}}=\kappa^{P} / \eta$ vs Péclet number, from Brownian Dynamics with $N=1000$ for $\phi=0.1$ to $\phi=0.55$. . . . . . . . . . .

6.9 The nondimensional hard sphere bulk viscosity $\overline{\kappa^{P}}=\kappa^{P} / \eta$ normalized with $\phi^{2} g_{0}(2 ; \phi)$ vs Péclet number, from Brownian Dynamics with $N=1000$ for $\phi=0.1$ to $\phi=0.55$. 
xvii

6.10 The nondimensional hard sphere bulk viscosity $\overline{\kappa^{P}}=\kappa^{P} / \eta$ normalized with $\phi^{2} g_{0}(2 ; \phi)$ and scaled with the time-scale for stress relaxation given by $\hat{D}_{N H}(\phi)$ vs the scaled Péclet number, from Brownian Dynamics with $N=1000$ for $\phi=0.1$ to $\phi=0.55$. . .

6.11 The nondimensional hard sphere bulk viscosity $\overline{\kappa^{P}}=\kappa^{P} / \eta$ normalized with $\phi^{2} g_{0}(2 ; \phi)$ and scaled with the time-scale for stress relaxation given by $\hat{D}_{N H}(\phi)$ vs the scaled Péclet number, from Brownian Dynamics with $N=100$ for $\phi=0.1$ to $\phi=0.55$. . .

6.12 Nondimensional Brownian contribution to the pressure in compression vs Péclet number, from Accelerated Stokesian Dynamics — near field with $N=100$ for $\phi=0.1$ to $\phi=0.5$. The open circles denote the equilibrium particle contribution

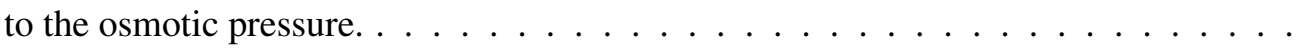

6.13 The nondimensional Brownian bulk viscosity contribution $\overline{\kappa^{B}}=\kappa^{B} / \eta$ vs Péclet number, from Accelerated Stokesian Dynamics — near field with $N=100$ for $\phi=0.1$ to

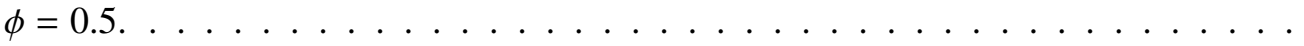

6.14 The nondimensional Brownian bulk viscosity contribution $\overline{\kappa^{B}}=\kappa^{B} / \eta$ normalized with $\phi^{2} g_{0}(2 ; \phi) / D_{0}^{s}(\phi)$ vs Péclet number, from Accelerated Stokesian Dynamics -

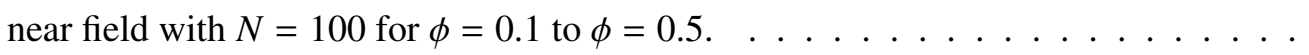

6.15 The nondimensional Brownian bulk viscosity contribution $\overline{\kappa^{B}}=\kappa^{B} / \eta$ normalized with $\phi^{2} g_{0}(2 ; \phi) / D_{0}^{s}(\phi)$ and scaled with the time-scale for stress relaxation given by $\hat{D}_{H}(\phi)$ vs the scaled Péclet number, from Accelerated Stokesian Dynamics — near field with $N=100$ for $\phi=0.1$ to $\phi=0.5 \ldots \ldots \ldots \ldots \ldots$

6.16 The nondimensional Brownian bulk viscosity contribution $\overline{\kappa^{B}}=\kappa^{B} / \eta$ normalized with $\phi^{2} g_{0}(2 ; \phi) / D_{0}^{s}(\phi)$ and scaled with the time-scale for stress relaxation given by $\hat{D}_{H}(\phi)$ vs the scaled Péclet number, from conventional Stokesian Dynamics with $N=$

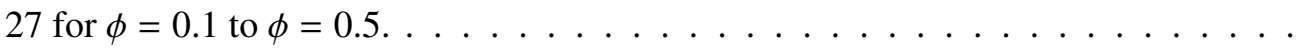

6.17 The nondimensional hydrodynamic bulk viscosity contribution $\overline{\kappa^{H}}=\kappa^{H} / \eta$ vs Péclet number, from Accelerated Stokesian Dynamics — near field with $N=100$ for $\phi=$ 0.1 to $\phi=0.5 \ldots \ldots \ldots \ldots \ldots \ldots \ldots$. . . . . . . . . . . . . . . . . . . . . . . . .

6.18 The normalized excess hydrodynamic bulk viscosity contribution $\left(\kappa^{H}(P e)-\kappa^{H}(0)\right) / \kappa^{H}(0)$ scaled with $g_{0}(2 ; \phi)$ vs Péclet number, from Accelerated Stokesian Dynamics — near field with $N=100$ for $\phi=0.1$ to $\phi=0.5 \ldots \ldots \ldots$. . . . . . . . . . 198 
xviii

6.19 The normalized excess hydrodynamic bulk viscosity contribution $\left(\kappa^{H}(P e)-\kappa^{H}(0)\right) / \kappa^{H}(0)$ scaled with $g_{0}(2 ; \phi)$ and the time-scale for stress relaxation given by $\hat{D}_{H}(\phi)$ vs the scaled Péclet number, from Accelerated Stokesian Dynamics — near field with $N=$ 100 for $\phi=0.1$ to $\phi=0.5 \ldots \ldots \ldots$. . . . . . . . . . . . . . . 199

6.20 The normalized excess hydrodynamic bulk viscosity contribution $\left(\kappa^{H}(P e)-\kappa^{H}(0)\right) / \kappa^{H}(0)$ scaled with $g_{0}(2 ; \phi)$ and the time-scale for stress relaxation given by $\hat{D}_{H}(\phi)$ vs the scaled Péclet number, from conventional Stokesian Dynamics with $N=27$ for $\phi=$

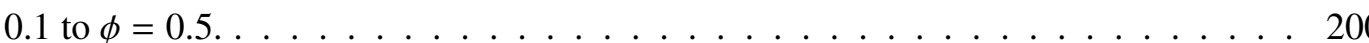

6.21 The total bulk viscosity contribution due to particle interactions $\overline{\kappa^{t o t}}=\left(\kappa^{H}+\kappa^{B}\right) / \eta$ vs Péclet number, from Accelerated Stokesian Dynamics — near field with $N=100$

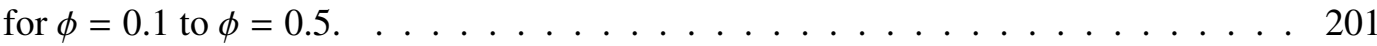

6.22 The total bulk viscosity at equilibrium and in compression with $P e=-1000$, vs the volume fraction $\phi$. In both cases the scaling with $\phi$ is the same and the difference in magnitude is not very significant. . . . . . . . . . . . . . . . . . . 202

6.23 The hydrodynamic contribution to the bulk viscosity, also known as the high-frequency bulk viscosity $\left(\kappa_{\infty}^{\prime}\right)$, for a sheared suspension vs the Péclet number of shearing. . . . 203

6.24 The effect of shearing on the high-frequency bulk viscosity $\left(\kappa_{\infty}^{\prime}\right)$ scaled with the effect of shearing on the high-frequency dynamic viscosity $\left(\eta_{\infty}^{\prime}\right)$ vs the volume fraction $\phi$. . 204

7.1 The suspending fluid is squeezed out as the filters enclosing a suspension are pushed closer causing compression of the particle phase as they are pushed closer to each other.216 


\section{List of Tables}

5.1 Equilibrium Osmotic Pressure from Brownian Dynamics simulations. . . . . . . . 152

$5.2 \quad$ Equilibrium Osmotic Pressure from ASDB-nf simulations. . . . . . . . . . . . 153

5.3 Relaxation time-scale for the shear stress autocorrelation function from Brownian Dynamics simulations with $N=1000$ particles. . . . . . . . . . . . . . 153

5.4 Relaxation time-scale for pressure autocorrelation function from Brownian Dynamics simulations with $N=1000$ particles. . . . . . . . . . . . . . . . . . 154

5.5 Relaxation time-scale for pressure autocorrelation function from Accelerated Stokesian Dynamics — near field simulations with $N=1000$ particles. . . . . . . . . . 155

5.6 Equilibrium (Green-Kubo) bulk viscosity for hard spheres from Brownian Dynamics simulations. . . . . . . . . . . . . . . . . . 156

5.7 Equilibrium (Green-Kubo) bulk viscosity for hard spheres with hydrodynamic interactions from ASDB-nf simulations. . . . . . . . . . . . . . . . . 157

5.8 Equilibrium (Green-Kubo) bulk viscosity for hard spheres with hydrodynamic interactions from conventional Stokesian Dynamics simulations. . . . . . . . . . 158

5.9 High-frequency bulk modulus for hard spheres with hydrodynamic interactions from

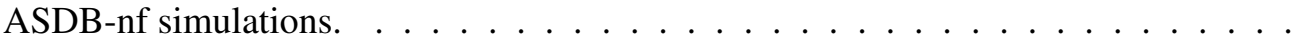

5.10 High-frequency bulk modulus for hard spheres with hydrodynamic interactions from conventional Stokesian Dynamics simulations. . . . . . . . . . . . . . . . 160

5.11 High-frequency bulk viscosity for hard spheres with hydrodynamic interactions from

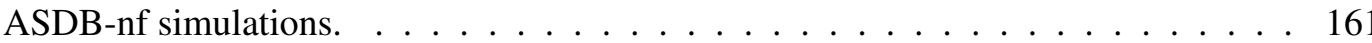

5.12 Equilibrium (Green-Kubo) shear viscosity for hard spheres from Brownian Dynamics simulations. . . . . . . . . . . . . . . . . . . . 162 
5.13 Equilibrium (Green-Kubo) shear viscosity for hard spheres with hydrodynamic interactions from ASDB-nf simulations. . . . . . . . . . . . . . . . . 162

6.1 The nondimensional particle-phase contribution to the osmotic pressure vs Pèclet number, from Brownian Dynamics simulations with $N=1000$, and 200 realizations

6.2 Error estimates $( \pm)$ for the nondimensional particle-phase contribution to the osmotic pressure vs Pèclet number, from Brownian Dynamics simulations with $N=1000$, and 200 realizations each. . . . . . . . . . . . . . . . . . 206

6.3 The nondimensional hard sphere bulk viscosity vs Pèclet number, from Brownian Dynamics simulations with $N=1000$, and 200 realizations each. . . . . . . . . 207

6.4 The nondimensional particle-phase contribution to the osmotic pressure vs Pèclet number, from Accelerated Stokesian Dynamics - near field simulations with $N=$ 100, and 200 realizations each. . . . . . . . . . . . . . . . . . 208

6.5 Error estimates $( \pm)$ for the nondimensional particle-phase contribution to the osmotic pressure vs Pèclet number, from Accelerated Stokesian Dynamics — near field simulations with $N=100$, and 200 realizations each. . . . . . . . . . . . . 209

6.6 The nondimensional Brownian contribution to the bulk viscosity with hydrodynamic interactions vs Pèclet number, from Accelerated Stokesian Dynamics — near field simulations with $N=100$, and 200 realizations each

6.7 The nondimensional Hydrodynamic bulk viscosity contribution $\overline{\kappa^{H}}=\kappa^{H} / \eta$ vs Pèclet number, from Accelerated Stokesian Dynamics - near field simulations with $N=$ 100, and 200 realizations each. . . . . . . . . . . . . . . . . . . . 211

6.8 Error estimates $( \pm$ ) for the nondimensional Hydrodynamic bulk viscosity contribution $\overline{\kappa^{H}}=\kappa^{H} / \eta$ vs Pèclet number, from Accelerated Stokesian Dynamics — near field

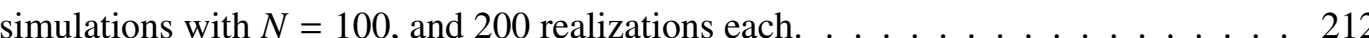




\section{Chapter 1}

\section{Introduction}

The stress in a material undergoing deformation can be thought of as consisting of a thermodynamic part, caused by inter-molecular forces that act to restore the original configuration of molecules in the material, and a viscous part, due to the energy spent as the molecules relax into a new configuration. In the case of an elastic solid, only the thermodynamic stress is present and it can be related to the deformation with the help of a Young's modulus for shear deformation and a Bulk modulus for volume change. At the other extreme are fluids, which do not exhibit elastic behavior, and therefore the stress is a function only of the rate of deformation of the fluid, in addition to the thermodynamic pressure. The stress in a pure isotropic fluid can be related to the rate of deformation with the help of two distinct coefficients of viscosity, or the transport coefficients. The shear viscosity $\eta$, which gives the stress in response to incompressible shearing flow, is the most commonly encountered transport coefficient. The bulk viscosity $\kappa$, also known as the second or expansion viscosity, is a measure of the resistance of a fluid to change in volume, in addition to the thermodynamic resistance. For example, when a fluid is expanding, the molecules are transformed into a less dense configuration. The force required, and therefore the energy spent, in causing the change in density, is given by the bulk viscosity. Specifically, the bulk viscosity relates the deviation of the normal stress from its equilibrium value to the rate of expansion or compression of the fluid.

The transport coefficients are vital for understanding the flow behavior of fluids. The bulk viscosity contribution to the fluid stress is significant only when there is a measurable unsteady volume-change in the fluid, given that it has a nonzero bulk viscosity coefficient. In most common applications, the effects of bulk viscosity are not important and consequently it is the least studied transport coefficient. However, the bulk viscosity of a fluid can be important when there 
is compression or expansion, such as in the absorption and dispersion of sound waves and indeed, experimental techniques to measure the bulk viscosity are often based on acoustic absorption measurements. The difference between the net absorption of acoustic energy and that predicted by classical theories based on thermal conductivity and shear viscosity gives the contribution due to bulk viscosity [Keizer 1987; Malbrunot et al. 1983].

Theoretical approaches for the calculation of bulk viscosity have made use of kinetic theory, statistical mechanics [Kirkwood et al. 1949] and molecular dynamics [Hoover et al. 1980]. All transport coefficients have been calculated for hard-sphere fluids by Sigurgeirsson and Heyes [2003] using analytical as well as numerical methods. The bulk viscosity can be calculated directly from the average decay of pressure variations in a molecular fluid by employing Green-Kubo relations [Green 1952]. Another approach is to use the definition of the bulk viscosity given by the linear thermodynamic force-flux relation [Eu 1998; Rah and Eu 1999]:

$$
\Delta \equiv \frac{1}{3} \boldsymbol{I}: \boldsymbol{\sigma}+p_{t h}=\kappa \boldsymbol{\nabla} \cdot \boldsymbol{u}
$$

where $\Delta$ is the excess normal stress, $\sigma$ is the stress in the fluid, $p_{t h}$ is the thermodynamic pressure, and $\boldsymbol{\nabla} \cdot \boldsymbol{u}$ is the expansion rate of the fluid. The excess normal stress is driven by compression or expansion of the fluid as shown in (1.1) and the bulk viscosity $\kappa$ is the constant of proportionality relating the two. In order to measure the bulk viscosity, the molecular fluid is subjected to a compressional/dilational disturbance such as a sound wave, which causes a density variation. The associated pressure variation is measured, and, along with knowledge of the equilibrium equation of state of the fluid, the bulk viscosity can be calculated from (1.1).

Now consider a system of neutrally buoyant particles suspended in a fluid. The interactions between the particles are governed by thermal, interparticle and hydrodynamic forces instead of inter-molecular forces. The equilibrium normal stress in the suspension is the sum of the osmotic pressure of the suspended particles and the fluid pressure. Let the particle microstructure - the distribution of particles in space and time - be subjected to a uniform compression or expansion. The resulting movement of particles will cause a disturbance flow in the fluid and there will be a corresponding change in the overall stress in the suspension determined by the thermodynamic and hydrodynamic interactions among the particles. The change in bulk normal stress as a result of the 
expansion gives rise to the bulk viscosity effect. The effective bulk viscosity for the suspension can therefore be calculated analogously to that of a hard-sphere fluid, by relating the deviation of the normal stress from its equilibrium value to the rate of compression/expansion of the particle microstructure.

One may wonder why the bulk viscosity should be important, when in most practical applications of suspensions both the fluid and the suspended particles are incompressible. Although the fluid and the particles may be not be compressible locally, when considered as a phase, both the fluid and particle phases are compressible. The particles and fluid may separate from each other in some regions and come closer in others, leading to local variations in particle volume fraction. When the particles are forced closer or pulled apart, the fluid between them gets squeezed out or in, respectively, and this squeezing motion generates an isotropic stress proportional to the rate of expansion, i.e., a bulk viscosity effect. Furthermore, the bulk viscosity would be important in the construction of two-phase flow equations for modeling certain suspension flows. For example, in filtration or compaction of a suspension the consolidation of the particle phase generates such a squeezing (or compressive) flow, which contributes to the bulk stress. Mathematically, a term proportional to $\boldsymbol{\nabla} \cdot \boldsymbol{u}_{p}$, where $\boldsymbol{u}_{p}$ is the particle phase velocity, must be added to the momentum balance. The coefficient of this term will be the effective bulk viscosity. The particle-phase momentum balance combined with the particle-phase continuity equation gives a diffusion equation for the macroscopic particle concentration [Nott and Brady 1994; Fang et al. 2002]. The addition of the bulk viscosity term to the bulk stress will add a non-diffusive term to the particle concentration equation thereby changing its mathematical type, and the temporal evolution of the concentration profile will now be different. The bulk viscosity term has important implication for modeling unsteady flows in suspensions, especially when there are rapid variations in particle concentration.

Bulk viscosity effects have been observed in many particulate systems where particles interact via inertial or other inter-particle forces. Kinetic-theory based models that are used to describe the rapid flow of granular media contain a contribution from the bulk viscosity, although the particles and fluid making up the fluid are individually incompressible [Lun et al. 1984; Gidaspow 1994]. There are also well established expressions for the bulk viscosity of molecular fluids [Sigurgeirsson and Heyes 2003]. Why should the situation be any different in a viscous suspension? There are inter-particle interactions between particles in a suspension just like in granular flows or molecular 
fluids, except that the forces are transmitted via viscous fluid flows. In the colloidal regime the microscale expressions for the shear viscosity terms are almost identical to those for molecular fluids. Therefore, we expect that there should also be a contribution to the bulk viscosity in a suspension. The only previous study on the bulk viscosity of two-phase materials that we are aware of was done by G. I. Taylor, in which he determined the bulk viscosity of a dilute suspension of bubbles expanding in an incompressible fluid by using energy dissipation arguments [Taylor 1954a,b]. We will show that the same result can be derived from mechanical arguments using the framework we develop for determining the bulk viscosity of hard sphere suspensions. The focus of the current study however, is on the complementary problem of rigid particles in a compressible fluid.

From a theoretical perspective the bulk viscosity is the third kind of viscosity characterizing the stress in a suspension. The suspension shear viscosity, which has been studied extensively, corresponds to a quadrupolar distortion of the microstructure (configuration of particles at the microscale) proportional to the deviatoric part of the rate-of-strain tensor. The 'microviscosity' which gives the resistance to a particle moving in a suspension and hindered by the surrounding particles (equivalent to the self-diffusion coefficient) [Khair and Brady 2006] has a dipolar forcing due to the distortion of the microstructure along the line of motion. The bulk viscosity, as we shall show, has a monopolar forcing because the disturbance in the microstructure is isotropic and proportional to the trace of the rate-of-strain tensor. Thus the computation of the bulk viscosity completes the set of possible rheological problems. Furthermore, a new hydrodynamic interaction function is needed for computing the bulk viscosity and it has been determined in a related work [Khair et al. 2006], which finally completes the set of hydrodynamic resistance and mobility functions for spherical particles. The main purpose of this work is to extend the existing theoretical framework for computing the transport properties of suspensions to include the effective bulk viscosity, and to determine the bulk viscosity for all concentrations and all rates of compression/expansion of the particle phase.

We begin with determination of the effective bulk viscosity in the dilute limit for all rates of expansion and compression in Chapter 2. The primary effect of particles suspended in an expanding fluid is an $O(\phi)$ (where $\phi$ is the volume fraction of particles) contribution to the bulk viscosity arising from the stress due to a disturbance flow caused by the rigid boundary at the surface of the particle. The particle surface cannot expand with the surrounding fluid so there must be a dis- 
turbance flow to satisfy the no-slip condition at the surface. The disturbance velocity satisfies the incompressible Stokes equations and therefore the correction to the bulk viscosity is proportional to the fluid shear viscosity $\eta$ and may not be negligible even for very dilute suspensions, depending on the magnitude of the fluid's bulk viscosity $\kappa$ in comparison to $\eta$. In order to compute the correction to the bulk viscosity due to interactions between particles it is necessary to determine the suspension microstructure and how it is influenced by the imposed expansion flow. The particle microstructure evolves from a competition between the imposed flow which drives the microstructure away from its equilibrium state and the restorative Brownian motion which acts to restore equilibrium. These competing effects are influenced by hydrodynamic interactions between the particles. The relative importance of the expansion flow to the restorative Brownian motion is given by the Péclet number $(\mathrm{Pe})$ which is defined as the bulk rate of expansion nondimensionalized with the time-scale of Brownian motion. The Péclet number can be positive or negative corresponding to expansion or contraction flow, respectively. For dilute suspensions the disturbance in the microstructure was obtained by solving the pair Smoluchowski equation to determine the pair-distribution function $g(r, t)$, where $r$ is the distance between the centers of two particles. The pair distribution function is normalized with the time-dependent average particle number density $n(t)$ so that the spatial perturbation in the microstructure can be isolated. For small Péclet numbers the disturbance to the microstructure was found to decay radially as $1 / r$ as $r \rightarrow \infty$, which can be attributed to the monopolar nature of the forcing in expansion flow. The two-particle stresses were then averaged over the microstructure to get the suspension bulk stress, and explicit expressions were derived for the bulk viscosity.

In Chapter 3 we study the time-dependent behavior of the suspension microstructure and the bulk stress in an expansion flow, and determine the bulk-viscoelastic rheology of suspensions. The monopolar nature of the forcing in expansion flow is also related to the temporal response of the suspension, and manifests as a slow $t^{-3 / 2}$ long-time decay of the Particle-pressure autocorrelation function at equilibrium. This connection was established by studying the bulk-viscoelasticity of the suspension in the linear-response regime by considering a rate of expansion that is uniform in space but oscillatory in time. The Péclet number is now based on the amplitude of the oscillatory expansion flow. The microstructural response is purely viscous in the zero-frequency limit corresponding to steady expansion/contraction. The elastic response grows as the oscillation frequency $\omega$ increases from 0 and as $\omega \rightarrow \infty$ both the viscous and elastic response decay to zero. At very high frequencies 
the microstructure is barely perturbed because of the small amplitude of oscillation and so there is no Brownian contribution to the bulk viscosity; only the direct hydrodynamic contribution remains.

At high particle concentrations the many-body interactions between particles become important and lubrication interactions between nearly touching particles comprise the dominant contribution to the bulk stress, necessitating the use of numerical simulations to calculate the total stress in the suspension. Chapter 4 contains a description of how existing methods for the dynamic simulation of suspensions were modified to allow expansion and compression of the particle phase. The Stokesian Dynamics (SD) technique developed by Phillips et al. [1988]; Brady and Bossis [1988] has been successfully employed for simulation of colloidal suspension for various flow regimes. However, so far it has been restricted to simulation of incompressible flows only. In order to compute the bulk viscosity from simulations the SD technique was adapted to allow for a uniform linear expansion flow and to compute the trace of the stress tensor for determining the particle-phase pressure. The Accelerated Stokesian Dynamics (ASD) [Sierou and Brady 2001] and the ASDB-nf [Banchio and Brady 2003] techniques developed more recently to speed up the simulations were also adapted for expansion flows. The updated simulation methods have enabled the study of a larger variety of suspension flows where the particle phase may undergo expansion or compression either by changing the number density of particle, or having the particles themselves expand or contract in addition to any other imposed forcing, with full hydrodynamic interactions between particles. In order to study the effect of hydrodynamic interactions on the microstructure Brownian Dynamics (SD) simulations were also performed for comparison.

In Chapter 5 we present our results for the equilibrium properties of Brownian suspensions for a wide range of concentrations. Numerical simulations of Brownian suspensions at equilibrium were performed using both $\mathrm{BD}$ and $\mathrm{ASD}$ to calculate the pressure autocorrelation function. In the absence of hydrodynamic interactions, scaling the results with the equilibrium pair-distribution function at contact $g(2 ; \phi)$ collapses all the simulation data onto a single curve. The scaled pressure autocorrelation data from our simulations matches very well with the analytical theory, thereby validating the theoretical work. However, the temporal decay is so slow that the data gets reduced to just noise and is unable to capture the $t^{-3 / 2}$ decay. Therefore it is important to know from theory how the long-time tails decay so that we can integrate the pressure autocorrelation function correctly to find the bulk viscosity. The rate of decay of the stress autocorrelation functions was found to scale as the 
single particle Stokes-Einstein-Sutherland diffusivity at small concentrations and with the long-time self-diffusivity at volume fractions greater than $35 \%$. The long-time self-diffusivity affects the rate of stress relaxation only when there is some caging of the particles at high concentrations. Knowing the scaling of the pressure autocorrelation function the dilute theory results for the bulk viscosity can be extrapolated for all concentrations simply by multiplying with $g(2 ; \phi)$. In the presence of hydrodynamic interactions the self-diffusivity of the particles also changes with concentration and an additional scaling with the short-time self diffusivity is introduced. The direct hydrodynamic contribution to the bulk viscosity was also computed by averaging over a large number of equilibrium particle configurations.

Dynamic simulations of compression flow in suspensions were performed for a range of Péclet number and the methodology and results are presented in Chapter 6. Compression of the particle phase at high $P e$ causes the formation of an isotropic boundary layer of size $1 / P e$ around the particles in which there is an $O(P e)$ accumulation of particles as they are pushed towards each other and the compression flow is balanced by Brownian diffusion of the particles. Lubrication forces between particles in the boundary layer constitute the dominant contribution to the stress causing a compression 'thickening' of the bulk viscosity at high $\mathrm{Pe}$. Simulation of a compression flow implies that the volume fraction of particles keeps changing with time, making it non-trivial to gather data for any given $\phi$ from the numerical data. To overcome this problem a large number of simulations were performed for each value of $P e$ and the rheological properties were averaged over all the runs and also over some neighboring time steps in order to get good statistics. Further, the microstructure may not have reached steady state at the time of interest even though we start the simulation at very small volume fractions. We found that steady state was reached at high Péclet numbers while at lower values of $P e$ a steady state could not be reached. With the appropriate scaling all the simulation data collapsed on the dilute theory prediction, especially in the large $P e$ limit, thus providing valuable insight into the physical processes that determine the microstructure and stress in compression flow of concentrated suspensions. In most practical situations the suspension would also undergo shearing motion in addition compression or expansion. Hence we also studied the bulk viscosity in a steadily sheared suspension.

Finally we conclude in Chapter 7 with comments on the importance and validity of our results and possible experimental setups that could be used to measure the bulk viscosity. Macroscopic 
equations for the modeling of suspension flows with the bulk viscosity term are also derived and used to simulate simple one-dimensional compression of a suspension to demonstrate bulk viscosity effects in a macroscopic flow. The bulk viscosity adds an additional diffusive term to the momentum balance for the particle phase, and therefore affects the temporal evolution of the concentration profile on a macroscopic scale. 


\section{Chapter 2}

\section{Dilute Theory}

Understanding the rheological properties of suspensions is a vital step in designing efficient equipment for numerous industrial applications and also for modeling many important natural phenomena which involve fluid-particle flows. Although extensive research has been carried out on the macroscopic rheological properties of suspensions [Batchelor 1974; Phillips et al. 1988; Russel et al. 1989], the effective bulk viscosity has largely been neglected, probably because of the non-obvious way in which such effects are manifested. For a pure fluid, the bulk viscosity $\kappa$, also known as the second or expansion viscosity, relates the deviation of the normal stress from its equilibrium value to the rate of expansion or compression of the fluid. The bulk viscosity measures the energy dissipated in causing a change in the fluid's density. For a suspension the effective bulk viscosity is defined analogously to that of a pure fluid as the constant of proportionality relating the deviation of the trace of the average macroscopic stress from its equilibrium value to the average rate of expansion. The effective bulk viscosity is then a measure of the energy dissipated in changing the number density (or volume fraction) of the particulate phase. Although the fluid and the particles may be not be compressible individually, when considered as a phase, both the fluid and particle phases are compressible. When the particles are forced closer or pulled apart, the fluid between them gets squeezed out or in, respectively, and this squeezing motion generates an isotropic stress proportional to the rate of expansion, i.e., a bulk viscosity effect. As a consequence, the bulk viscosity may play an important role in the behavior of suspensions, especially when there is a rapid and sharp variation in particle concentration - shocks in particle volume fraction - which occur frequently in suspension flows.

The effective macroscopic properties, e.g., viscosity, sedimentation rates, diffusion coefficients, 
etc., of suspensions, have been studied extensively over a period of several decades. A detailed program for relating the macroscopic properties of two-phase materials to the underlying microscale physics when fluid mechanical interactions are important was laid out by G. K. Batchelor and coworkers [Batchelor 1974]. They established the averaging procedure to pass from the microscale to the macroscale [Batchelor 1970], determined the relevant particle interactions at the microscale, e.g., for the shear viscosity [Batchelor and Green 1972a], and showed how the microstructure of the suspension - the spatial and temporal distribution of particles - must be determined as part of the problem in order to compute the averaged properties [Batchelor and Green 1972b; Batchelor 1977]. Although most of Batchelor's results were limited to dilute suspensions, the procedure laid out by him can be applied to all concentrations. Recent advances in computational techniques have enabled the extension of this method to higher particle concentrations, even all the way up to close packing [Brady and Bossis 1988; Phillips et al. 1988; Ladd 1990; Sierou and Brady 2001].

We followed the procedure laid out by Batchelor to determine the effective bulk viscosity of a suspension of rigid spherical particles. Prior to this work, the only study of the bulk viscosity of two-phase materials appears to be that of G. I. Taylor, in which he determined the bulk viscosity of a dilute suspension of bubbles expanding in an incompressible fluid by using energy dissipation arguments [Taylor 1954a,b]. We were able to show that our approach of computing the bulk viscosity directly from the average macroscopic stress leads to the same result as Taylor's for bubbles. The focus of our study is, however, on the complementary problem of rigid particles in a compressible fluid.

This chapter is organized as follows. In $§ 2.1$ we recall the definition of the bulk viscosity for a pure compressible fluid as the constant relating the difference between the mechanical and thermodynamic pressures to the rate of expansion. We then consider how the presence of particles in the fluid affects the bulk viscosity. In $\S 2.2$ the effective bulk viscosity for a suspension is defined from mechanical stress considerations. The expressions derived here can in principle be used to calculate the bulk viscosity for all concentrations. In $\S 2.3$ we compute the $O\left(\phi^{2}\right)$ correction to bulk viscosity which arises from particle-pair interactions. Equations are derived to describe the pair-distribution function for identical Brownian particles in a uniform expansion flow. The particles interact via an excluded-volume interparticle potential whose range can be varied to study the influence of hydrodynamic interactions in a simple, systematic manner. The equations are solved numerically in $§ 2.4$ 
and the resulting average stresses due to pair-interactions are used to determine the bulk viscosity. Concluding remarks are given in $§ 2.5$.

\subsection{Measurement of the bulk viscosity}

The stress in a pure fluid is comprised of the thermodynamic pressure and the mechanical stress originating from deformation of the fluid. For a Newtonian fluid, the mechanical stress is a linear function of the rate of deformation and the total fluid stress can be written as

$$
\boldsymbol{\sigma}=-p_{t h} \boldsymbol{I}+2 \eta \boldsymbol{e}+\left(\kappa-\frac{2}{3} \eta\right)(\boldsymbol{\nabla} \cdot \boldsymbol{u}) \boldsymbol{I},
$$

where $p_{t h}$ is the thermodynamic pressure, $\boldsymbol{I}$ is the isotropic tensor, $\eta$ is the shear viscosity and

$\boldsymbol{e}=\frac{1}{2}\left[\boldsymbol{\nabla} \boldsymbol{u}+(\boldsymbol{\nabla} \boldsymbol{u})^{\dagger}\right]$ is the rate-of-strain tensor with $\boldsymbol{u}$ being the fluid velocity. When the fluid is at rest, the stress is isotropic and equal to the thermodynamic pressure. The bulk viscosity $\kappa$ is the constant of proportionality which relates the difference between the mechanical pressure, defined as the mean mechanical stress, and the thermodynamic pressure to the rate of volume expansion, $\boldsymbol{\nabla} \cdot \boldsymbol{u}$, of a fluid undergoing deformation:

$$
p_{\text {mech }} \equiv-\frac{1}{3} \boldsymbol{I}: \boldsymbol{\sigma}=p_{t h}-\kappa \boldsymbol{\nabla} \cdot \boldsymbol{u} .
$$

If the fluid is incompressible, $\boldsymbol{\nabla} \cdot \boldsymbol{u}=0$, the mechanical and thermodynamic pressures are the same, and the mechanical pressure is known to within an arbitrary constant. The bulk viscosity is a measure of dissipation of energy in the fluid upon expansion.

Although experimental measurements of the bulk viscosity of a pure fluid are often made by studying the absorption of acoustic waves in which there is a series of compressions and expansions, one could have an experiment in which there is only expansion of the fluid at a given rate $e(=\boldsymbol{\nabla} \cdot \boldsymbol{u})$, and measure the mechanical pressure. The thermodynamic pressure of the fluid can be calculated from its equation of state and the difference between the mechanical and thermodynamic pressures gives the bulk viscosity times the rate of expansion.

A similar experiment can be carried out for measuring the bulk viscosity of a suspension in which the fluid expands at a constant rate causing the particles also to move apart and the suspen- 
sion to expand. Since the particles do not expand at the same rate as the fluid, they will create a disturbance flow and thus dissipate more energy than the fluid would alone. The excess dissipation is manifested as a change in the effective bulk viscosity of the material. The difference between the mechanical pressure in the system and the equilibrium pressure gives the effective bulk viscosity times the average rate of expansion of the suspension. We follow this approach to determine the effective bulk viscosity.

While the above procedure completely defines and determines the bulk viscosity, its application to suspension flows requires some comment. First, one could imagine carrying out just such an experiment as outlined above - expand uniformly a fluid and measure the mechanical pressure, then repeat the process with suspended particles. The difference between the two measurements gives the particles' contribution to the bulk viscosity. However, in many applications the particles and fluid are incompressible and therefore carrying out such a procedure could be difficult. Instead, one can exploit the compressibility of the particle phase (not the particles themselves) to define a bulk viscosity. Compressing the particle phase will also generate a mechanical pressure proportional to the rate of expansion (or compression) and thus a bulk viscosity effect. However, such a procedure necessarily generates a spatial variation in particle fraction, which then complicates the determination of the bulk viscosity as a function of particle volume fraction. The approach taken here of expanding the fluid allows us to pose a spatially homogeneous problem for determining the bulk viscosity, and one expects the bulk viscosity determined by both approaches to be comparable.

\subsection{Bulk viscosity of a suspension}

Consider a homogeneous suspension of spherical particles with number density $n$ in a compressible Newtonian fluid of density $\rho$, shear viscosity $\eta$ and bulk viscosity $\kappa$. The particles are small enough so that the Reynolds number $R e=\rho U a / \eta$ (with $U$ being a typical velocity and $a$ being the radius of the particles) is much less than unity, thus enabling the use of Stokes equations. The fluid is made to expand everywhere in space at a uniform rate $e(=\boldsymbol{\nabla} \cdot \boldsymbol{u})$. The imposed flow will cause the suspended particles to move apart but they cannot expand with the fluid because they are rigid. There will be a disturbance flow as the fluid has to move around the particles to compensate for their rigidity and this disturbance flow will cause the stress on the particles to change, thereby affecting 
the bulk stress in the suspension. A uniform compressive flow (negative expansion rate) could be assumed as well without affecting the following derivation.

The bulk viscosity of the suspension is determined by computing the average stress in the material in a way analogous to that for the shear viscosity [Batchelor and Green 1972b; Brady and Bossis 1988; Brady et al. 2006]. The ensemble or volume average of the Cauchy stress $\sigma$ in the material is given by

$$
\langle\boldsymbol{\sigma}\rangle=-\left\langle p_{t h}\right\rangle_{f} \boldsymbol{I}+2 \eta\langle\boldsymbol{e}\rangle+\left(\kappa-\frac{2}{3} \eta\right)\langle\boldsymbol{\nabla} \cdot \boldsymbol{u}\rangle \boldsymbol{I}+n\left\langle\boldsymbol{S}^{H}\right\rangle,
$$

where $\boldsymbol{e}$ is the rate of strain in the fluid, $\langle\ldots\rangle$ denotes an average over the entire suspension (particles plus fluid), and $\langle\ldots\rangle_{f}$ denotes an average over the fluid phase only. The average hydrodynamic stresslet is defined as a number average over all particles by $\left\langle\boldsymbol{S}^{H}\right\rangle=(1 / N) \sum_{\alpha=1}^{N} \boldsymbol{S}_{\alpha}^{H}$, where the stresslet of particle $\alpha$ is given by

$$
\boldsymbol{S}_{\alpha}^{H}=\frac{1}{2} \int_{S_{\alpha}}\left[(\boldsymbol{r} \boldsymbol{\sigma} \cdot \boldsymbol{n}+\boldsymbol{\sigma} \cdot \boldsymbol{n r})-2\left(\kappa-\frac{2}{3} \eta\right)(\boldsymbol{n} \cdot \boldsymbol{u}) \boldsymbol{I}-2 \eta(\boldsymbol{u} \boldsymbol{n}+\boldsymbol{n u})\right] \mathrm{d} S .
$$

The particle stresslet is the symmetric part of the first moment of the surface stress on the particle. The integral is over the surface of particle $\alpha$ with normal $\boldsymbol{n}$ pointing into the fluid and $\boldsymbol{r}$ is the spatial vector from the center of the particle to a point on its surface. In writing (2.4) it has been assumed that there is no net force on the particle; an assumption that is relaxed below.

The stress resulting from Brownian motion of the particles as well as an interparticle-force contribution $-n\left\langle\boldsymbol{x} \boldsymbol{F}^{P}\right\rangle$ must also be added to the bulk stress. In the present study a simple hardsphere interparticle potential is assumed that keeps the particles from overlapping. Inertial Reynolds stresses could also be added, but since our interest is in low-Reynolds-number flows only, we ignore the inertial part. The final form of the bulk stress can be written as

$$
\langle\boldsymbol{\Sigma}\rangle=-\left\langle p_{t h}\right\rangle_{f} \boldsymbol{I}+2 \eta\langle\boldsymbol{e}\rangle+\left(\kappa-\frac{2}{3} \eta\right)\langle\boldsymbol{\nabla} \cdot \boldsymbol{u}\rangle \boldsymbol{I}-n k T \boldsymbol{I}+n\left[\left\langle\boldsymbol{S}^{B}\right\rangle+\left\langle\boldsymbol{S}^{P}\right\rangle+\left\langle\boldsymbol{S}^{E}\right\rangle\right],
$$

where it is assumed that there are no external couples on the particles [Brady 1993a]. The averaged 
particle stresslets can be expressed in terms of hydrodynamic resistance functions as

$$
\begin{aligned}
& \left\langle\boldsymbol{S}^{B}\right\rangle=-k T\left\langle\boldsymbol{\nabla} \cdot \boldsymbol{R}_{S U} \cdot \boldsymbol{R}_{F U}^{-1}\right\rangle, \\
& \left\langle\boldsymbol{S}^{P}\right\rangle=-\left\langle\left(\boldsymbol{R}_{S U} \cdot \boldsymbol{R}_{F U}^{-1}+\boldsymbol{x} \boldsymbol{I}\right) \cdot \boldsymbol{F}^{P}\right\rangle, \\
& \left\langle\boldsymbol{S}^{E}\right\rangle=-\left\langle\boldsymbol{R}_{S U} \cdot \boldsymbol{R}_{F U}^{-1} \cdot \boldsymbol{R}_{F E}-\boldsymbol{R}_{S E}\right\rangle:\langle\boldsymbol{e}\rangle,
\end{aligned}
$$

where the derivative is with respect to the last index of the inverse of the resistance matrix $\boldsymbol{R}_{F U}^{-1}$ and $\boldsymbol{F}^{P}$ is the colloidal interparticle force.

The fluid velocity resulting from a uniform rate of expansion can be decomposed into a uniform expansion and a disturbance (Stokes) velocity

$$
\boldsymbol{u}=\frac{1}{3} e \boldsymbol{r}+\boldsymbol{u}^{s}
$$

such that

$$
\boldsymbol{\nabla} \cdot \boldsymbol{u}=e \quad \text { and } \quad \boldsymbol{\nabla} \cdot \boldsymbol{u}^{s}=0
$$

The disturbance flow created by the particles and the resulting fluid mechanical interactions satisfy the usual incompressible equations of motion. The fluid stress associated with the uniform expansion flow is

$$
\boldsymbol{\sigma}^{e}=-\left(p_{t h}-\kappa e\right) \boldsymbol{I}
$$

while the disturbance stress is given by

$$
\boldsymbol{\sigma}^{s}=-p^{s} \boldsymbol{I}+2 \eta \boldsymbol{e}^{s} \quad \text { and satisfies } \quad \boldsymbol{\nabla} \cdot \boldsymbol{\sigma}^{s}=0,
$$

where $p^{s}$ is the dynamical pressure distribution associated with the incompressible disturbance Stokes flow. Note that since the disturbance flow $\left(\boldsymbol{u}^{s}, \boldsymbol{\sigma}^{s}\right)$ satisfies the incompressible Stokes equation, the hydrodynamic interaction functions in (2.6), e.g. $\boldsymbol{R}_{F U}$, are the usual ones for incompressible flow.

The suspension as a whole has a uniform average rate of expansion $\langle e\rangle \equiv\langle\boldsymbol{\nabla} \cdot \boldsymbol{u}\rangle$, where the averaging is done over the fluid and the particles; for rigid particles $\langle e\rangle=(1-\phi) e$. The bulk 
viscosity is the scalar coefficient that when multiplied with the average rate of expansion gives the difference between the mean suspension stress and the equilibrium stress. The suspension will be in equilibrium when $\langle\boldsymbol{e}\rangle \equiv 0$ and in that case, the bulk stress is given by

$$
\langle\boldsymbol{\Sigma}\rangle^{e q}=-\left(\left\langle p_{t h}\right\rangle_{f}^{e q}+\Pi\right) \boldsymbol{I},
$$

where $\Pi$ is the osmotic pressure:

$$
\Pi=n k T-\frac{1}{3} n\left[\left\langle S^{B}\right\rangle^{e q}+\left\langle S^{P}\right\rangle^{e q}\right],
$$

and $S$ denotes the trace of the corresponding stresslet, as in $\left\langle\boldsymbol{S}^{B}\right\rangle^{e q}=\frac{1}{3}\left\langle S^{B}\right\rangle^{e q} \boldsymbol{I}$, and the superscript $e q$ denotes an average over the equilibrium distribution of the suspension microstructure. The effective bulk viscosity $\kappa_{e f f}$ is therefore given by

$$
\kappa_{e f f} \equiv \kappa+\left(-\left\langle p_{t h}\right\rangle_{f}+\left\langle p_{t h}\right\rangle_{f}^{e q}\right) /\langle e\rangle+\frac{1}{3} n\left[\left(\left\langle S^{B}\right\rangle-\left\langle S^{B}\right\rangle^{e q}\right)+\left(\left\langle S^{P}\right\rangle-\left\langle S^{P}\right\rangle^{e q}\right)+\left\langle S^{E}\right\rangle\right] /\langle e\rangle .
$$

Equation (2.12) together with equation (2.6) gives the general expressions which can be used to calculate the effective bulk viscosity of a suspension for all concentrations and expansion or compression rates. The Brownian and interparticle force contributions arise from interactions between at least two particles and therefore contribute $O\left(\phi^{2}\right)$ to the bulk viscosity. The stresslet due to the imposed rate-of-strain $\left\langle S^{E}\right\rangle$ is nonzero for a single particle and therefore contributes $O(\phi)$ to the bulk viscosity.

The $O(\phi)$ contribution to bulk viscosity arises from the disturbance flow induced by the presence of a single particle suspended in the uniform expansion flow. Since the particle cannot expand with the fluid, the no-slip condition on its surface causes a disturbance flow:

$$
\boldsymbol{u}^{s}=-\frac{1}{3} e \frac{a^{3}}{r^{3}} \boldsymbol{r}
$$

The particle stresslet from (2.4) is

$$
S^{E}=4 \pi a^{3}\left(-p_{t h}+\kappa e+\frac{4}{3} e \eta\right)
$$


and the effective bulk viscosity from (2.12) is to first order in $\phi$ :

$$
\begin{aligned}
\kappa_{e f f} & =\left(\kappa+\frac{4}{3} \eta \phi\right) \frac{1}{1-\phi}, \\
& \sim \kappa+\left(\kappa+\frac{4}{3} \eta\right) \phi \quad \text { as } \quad \phi \rightarrow 0,
\end{aligned}
$$

The $\frac{4}{3} \eta \phi$ term corresponds to the 'Einstein' correction to the bulk viscosity for a dilute suspension of rigid spheres [Brady et al. 2006]. The factor of $1 /(1-\phi)$ represents the difference between the fluid and the bulk's rate of expansion. The correction to the bulk viscosity is proportional to the shear viscosity $\eta$ and therefore may not be negligible even for very dilute suspensions, depending on the magnitude of the fluid's bulk viscosity $\kappa$ in comparison to $\eta$.

The same formulation can be used to recover G. I. Taylor's result for the bulk viscosity of a dilute suspension of bubbles. In this case the suspending fluid is incompressible and the bubbles are compressible. Consider a single bubble of radius $a$, bulk viscosity $\kappa_{p}$, and zero shear viscosity in an incompressible fluid expanding uniformly with rate $e_{p}$. Since only the volume occupied by the bubble is expanding the average rate of expansion in the dispersion is $\langle e\rangle=e_{p} \phi$. The expanding bubble surface creates an incompressible disturbance flow in the surrounding fluid

$$
\boldsymbol{u}^{s}=\frac{1}{3} e_{p}\left(a^{3} / r^{3}\right) \boldsymbol{r}
$$

In contrast to the rigid particles problem the bubbles cause the pressure in the surrounding fluid to change from its equilibrium value. The fluid pressure is determined through a normal stress balance on the surface of the bubble, $\left(\sigma_{p}-\boldsymbol{\sigma}\right) \cdot \boldsymbol{n}=0$ (neglecting surface tension effects, which can be added but do not alter the final result anyway), and is given by

$$
p_{t h}=p_{p}-\kappa_{p} e_{p}-\frac{4}{3} \eta e_{p},
$$

where $p_{p}$ is the equilibrium pressure inside the bubble and equal to the equilibrium pressure in the surrounding fluid neglecting the capillary pressure due to surface tension. The resulting stresslet on the bubble is

$$
S^{E}=4 \pi a^{3}\left(-p_{p}+\kappa_{p} e_{p}+\frac{4}{3} \eta e_{p}\right)
$$


and from (4.10) the effective bulk viscosity for the dispersion is found to be

$$
\begin{aligned}
\kappa_{e f f} & =\left[\kappa_{p} \phi+\frac{4}{3} \eta(1-\phi)\right] \frac{e_{p}}{\langle e\rangle} \\
& =\left[\kappa_{p} \phi+\frac{4}{3} \eta(1-\phi)\right] \frac{1}{\phi} \\
& \sim \kappa_{p}+\frac{4}{3} \eta / \phi \text { as } \phi \rightarrow 0 .
\end{aligned}
$$

Thus we get the correction to the bulk viscosity as $\frac{4}{3} \eta / \phi$, a result first derived by Taylor [1954a,b]. The inverse dependence on $\phi$ is unusual and entails further comment. The small $O(\phi)$ concentration of bubbles produces a small rate of expansion $\left(\langle e\rangle=\phi e_{p}\right)$ throughout the dispersion, however the pressure field is perturbed over the entire surrounding fluid, which occupies a large $O(1-\phi)$ fraction of the total volume. Thus the dominant contribution comes from the fluid pressure term $\left(-\left\langle p_{t h}\right\rangle_{f}+\left\langle p_{t h}\right\rangle_{f}^{e q}\right) /\langle e\rangle$ in (4.10), which is of $O((1-\phi) / \phi) \sim O(1 / \phi)$ as $\phi \rightarrow 0$. In contrast the change in the stresslet exerted by a rigid particle is localized to its surface and therefore contributes $O(\phi)$ to the total stress, while the expanding fluid gives an average rate of expansion of $O(1-\phi)$, resulting in a correction to the bulk viscosity of $O(\phi)$ as $\phi \rightarrow 0$. Both problems do have the same co-

efficient, namely $\frac{4}{3} \eta$ in the bulk viscosity correction because the disturbance flow in the surrounding fluid is the same in both cases but with opposite sign.

\subsection{The $O\left(\phi^{2}\right)$ correction to bulk viscosity}

The interaction between particle-pairs results in the $O\left(\phi^{2}\right)$ correction to the bulk viscosity, and the Brownian, interparticle-force and rate-of-strain stresslets may all contribute. There will be higherorder corrections as well, resulting from interactions between three or more particles, etc.; however, in the present study we limit ourselves to two-particle interactions only. It is necessary to determine the spatial and temporal distribution of particles - the suspension microstructure - and how it is influenced by the imposed expansion flow in order to compute the bulk viscosity.

\subsubsection{Pair-evolution equation}

In order to calculate the microstructure, the $N$-particle Smoluchowski equation for identical particles is integrated over $N-2$ particles [Russel et al. 1989] and results in the following pair-evolution 
equation after all three-particle effects are neglected:

$$
\frac{\partial P_{1 / 1}}{\partial t}+\boldsymbol{\nabla}_{r} \cdot\left[\boldsymbol{U}_{r}^{E}+\boldsymbol{M}_{r} \cdot\left(\boldsymbol{F}_{r}^{P}-k T \boldsymbol{\nabla}_{r} \ln P_{1 / 1}\right)\right] P_{1 / 1}=0
$$

where $P_{1 / 1}(\boldsymbol{r}, \boldsymbol{t})$ is the conditional probability density of finding a second particle at $\boldsymbol{r}$ relative to one at the origin, $\boldsymbol{U}_{r}^{E}=\boldsymbol{U}_{2}^{E}-\boldsymbol{U}_{1}^{E}$ is the relative velocity of the two particles owing to the expansion flow, $\boldsymbol{M}_{r}=2\left(\boldsymbol{M}_{22}-\boldsymbol{M}_{21}\right)$ is the relative mobility, $\boldsymbol{F}_{r}^{P}$ is the relative interparticle force and $\boldsymbol{r}=\boldsymbol{x}_{2}-\boldsymbol{x}_{1}$ is the relative separation vector. The boundary condition of no relative flux at contact is written as

$$
\hat{\boldsymbol{r}} \cdot \boldsymbol{D}_{r} \cdot \boldsymbol{\nabla}_{r} P_{1 / 1}=\hat{\boldsymbol{r}} \cdot\left[\boldsymbol{U}_{r}^{E}+\boldsymbol{M}_{r} \cdot \boldsymbol{F}_{r}^{P}\right] P_{1 / 1} \quad \text { at } \quad r=2 a,
$$

where $\hat{\boldsymbol{r}}$ denotes a unit vector and $\boldsymbol{D}_{r}=k T \boldsymbol{M}_{r}$ is the relative diffusivity of the pair. At large separations, the probability density of finding a second particle is just the number density, or

$$
P_{1 / 1} \sim n(t) \quad \text { as } \quad r \rightarrow \infty .
$$

The number density of particles is a function of time only, owing to the assumption of uniform expansion or contraction. The conservation equation for the number density for uniform bulk expansion is given by

$$
\frac{\partial n}{\partial t}=-\boldsymbol{\nabla} \cdot(\langle\boldsymbol{u}\rangle n)=-n \boldsymbol{\nabla} \cdot\langle\boldsymbol{u}\rangle=-n\langle e\rangle .
$$

As a result, the number density varies exponentially in time as $n(t)=n_{0} e^{-\langle e\rangle t}$.

The conditional probability density can be expressed in terms of of the number density and pair-distribution function as $P_{1 / 1}=n(t) g(\boldsymbol{r}, t)$, and the resulting equation for the pair-distribution function can be written as

$$
\frac{\partial g}{\partial t}+\frac{1}{3}\langle e\rangle \boldsymbol{r} \cdot \boldsymbol{\nabla} g+\boldsymbol{\nabla} \cdot\left(\hat{\boldsymbol{U}}^{E}+\boldsymbol{M} \cdot \boldsymbol{F}^{P}\right) g=\boldsymbol{\nabla} \cdot \boldsymbol{D} \cdot \boldsymbol{\nabla} g
$$

where $\hat{\boldsymbol{U}}^{E}=\boldsymbol{U}^{E}-\frac{1}{3}\langle e\rangle \boldsymbol{r}$ is the disturbance velocity relative to the imposed flow, and the subscript $r$ has been dropped for clarity. The contact boundary condition (2.21) remains the same for $g$, while the far-field boundary condition (2.22) now becomes the time-independent statement $g \sim 1$ as $r \sim \infty$. 
The particle microstructure evolves from a competition between the imposed flow and the restorative Brownian motion. The expansion flow drives the microstructure away from its equilibrium state and Brownian diffusion acts to restore the equilibrium. These competing effects are influenced by hydrodynamic interactions between the particles. The strength of the hydrodynamic interactions can tuned by using the 'excluded annulus' model where the two-body interparticle potential $V(r)$ is given by

$$
V(r)=\left\{\begin{array}{cc}
\infty & \text { if } r \leq 2 b \\
0 & \text { if } r>2 b
\end{array} .\right.
$$

The length $b(\geq a)$ is the excluded-volume, or thermodynamic radius of a particle and the center-tocenter separation between particles can be no less that $2 b$. This potential can be used to model interactions arising from conditions such as surface roughness, grafted polymer chains or electrostatic repulsion. Altering the tunable parameter $\hat{b} \equiv b / a$ allows one to examine the role of hydrodynamic interactions in determining the microstructure and the bulk viscosity. The particles experience no hydrodynamic interactions as $\hat{b} \rightarrow \infty$, while full hydrodynamic interactions between particles are present when $\hat{b} \equiv 1$. With the excluded-annulus model, the interparticle force is implemented through the no-flux boundary condition (2.22) at $r=2 b$ and does not appear explicitly in the pairevolution equation. The volume fraction of particles based on the excluded-volume radius $b$ now has to be small for the diluteness assumption to hold, i.e., $\phi_{b}=4 \pi n b^{3} / 3 \ll 1$.

The expansion flow is spherically symmetric, which allows us to write the relative particle velocity as

$$
\boldsymbol{U}^{E}=\frac{1}{3}\langle e\rangle\left(r+b v^{\prime}(r)\right) \hat{\boldsymbol{r}}
$$

where $v^{\prime}(r)$, a function of the scalar separation $r$, is the non-dimensional disturbance velocity relative to the imposed expansion flow. Similarly, the pair-distribution function will also be spherically symmetric. Consequently, the non-dimensional pair-evolution equation with dependence on only time and the scalar separation $r$ is

$$
\begin{gathered}
\frac{\partial g}{\partial t}+P e_{b} r \frac{\partial g}{\partial r}+P e_{b} \frac{1}{r^{2}} \frac{\partial}{\partial r} r^{2} v^{\prime}(r \hat{b}) g=\frac{1}{r^{2}} \frac{\partial}{\partial r} r^{2} G(r \hat{b}) \frac{\partial g}{\partial r}, \\
G(r \hat{b}) \frac{\partial g}{\partial r}=P e_{b}\left[2+v^{\prime}(2 \hat{b})\right] g \quad \text { at } \quad r=2,
\end{gathered}
$$


and

$$
g \sim 1 \quad \text { as } \quad r \rightarrow \infty,
$$

where $G(r \hat{b})=x_{22}^{a}-x_{21}^{a}$ [Kim and Karrila 1991] is the scalar function for the radial component of the relative mobility of two particles, $\boldsymbol{D}=2 D[G(r \hat{b}) \hat{\boldsymbol{r}} \hat{\boldsymbol{r}}+H(r \hat{b})(\boldsymbol{I}-\hat{\boldsymbol{r}} \hat{\boldsymbol{r}})]$ [Batchelor 1976]. It should be noted that the tabulated values of the hydrodynamic functions are scaled with the actual particle radius $a$, whereas the radial distance in the given equations has been scaled with the excludedvolume radius $b$. The relative diffusivity is scaled by its value at large separations, $2 D$. The Péclet number is also based on the excluded-volume radius and is given by

$$
P e_{b}=\frac{\frac{1}{3}\langle e\rangle b^{2}}{2 D}
$$

where $D=k T / 6 \pi \eta a$ is the diffusivity of an isolated particle. The time is nondimensionalized by the diffusive time $b^{2} / 2 D$. The Péclet number can be positive or negative corresponding to expansion or contraction, respectively.

A steady-state solution can be found for the perturbed state radial-distribution function when the expansion rate is negative $\left(P e_{b}<0\right)$. The compression flow brings the particles closer together, whereas Brownian and interparticle forces push them apart, resulting in an eventual steady-state of the pair-distribution function. The overall number density, of course, increases with time as prescribed by the compression flow. If the time scale for the evolution of the microstructure (diffusive time) is the same as the time scale for increase in number density $\left(\langle e\rangle^{-1}\right)$, the solution for $g(r, t)$ will not reach steady-state for a given volume fraction. Therefore care should be taken in defining the steady-state bulk viscosity.

To obtain the steady-state solution of the perturbed microstructure, we write

$$
g(r)=1+P e_{b} f(r),
$$

and the steady-state equation for $f(r)$ becomes

$$
G(r \hat{b}) \frac{\partial^{2} f}{\partial r^{2}}+\left[\frac{2 G(r \hat{b})}{r}+\frac{\partial G(r \hat{b})}{\partial r}-P e_{b}\left(r+v^{\prime}\right)\right] \frac{\partial f}{\partial r}-\left(\frac{2 v^{\prime}}{r}+\frac{\partial v^{\prime}}{\partial r}\right)\left(1+P e_{b} f\right)=0
$$


with boundary conditions

$$
G(2 \hat{b}) \frac{\partial f}{\partial r}=\left[2+v^{\prime}(2 \hat{b})\right]\left(1+P e_{b} f\right) \quad \text { at } \quad r=2,
$$

and

$$
f \sim 0 \quad \text { as } \quad r \rightarrow \infty .
$$

It should be noted that the disturbance to the pair-distribution function has merely been scaled by $P e_{b}$ so that the resulting solutions for $f(r)$ are comparable over a wide range of Péclet numbers. No terms have been neglected and therefore, the results obtained are the full solution for $f(r)$ (or $g(r)$ ).

An analytical solution was obtained for small values of $P e_{b}$ for either compression or expansion by Brady et al. [2006], which corresponds to the linear-response regime. In this regime, the disturbance to the microstructure has a linear dependence on the Péclet number. Only the $O(1)$ terms are kept in (2.27) (the $O\left(P e_{b}\right)$ terms are neglected), which results in a first-order differential equation for $\partial f / \partial r$ that can be solved easily. The analytical solution is given by the quadrature

$$
f(r)=-\int_{r}^{\infty} \frac{1}{G(r \hat{b})}\left[\frac{8}{r^{2}}+v^{\prime}(r \hat{b})\right] \mathrm{d} r
$$

and is valid for both expansion and compression when $\left|P e_{b}\right| \ll 1$. In the absence of hydrodynamics $\hat{b} \rightarrow \infty, v^{\prime}=0$ and the solution reduces to

$$
f_{N H}(r)=-\frac{8}{r}
$$

For larger values of the Péclet number the $O\left(P e_{b}\right)$ terms cannot be ignored in the equation for $f(r)$ and therefore due to loss of linearity we do not expect to get the same solution for expansion and compression.

Equation (2.27) can also be simplified for the case of no hydrodynamic interactions and an 
analytical solution valid for all negative Péclet numbers (compression flow) is given by

$$
\begin{aligned}
f_{N H}(r) & =\frac{-2 \int_{r}^{\infty} \frac{1}{r^{2}} e^{\frac{1}{2} P e_{b} r^{2}} \mathrm{~d} r}{\frac{1}{4} e^{2 P e_{b}}+2 P e_{b} \int_{2}^{\infty} \frac{1}{r^{2}} e^{\frac{1}{2} P e_{b} r^{2}} \mathrm{~d} r} \\
& =\frac{-\frac{2}{r} e^{\frac{1}{2} P e_{b} r^{2}}+\sqrt{-2 \pi P e_{b}} \operatorname{erfc}\left(r \sqrt{-\frac{P e_{b}}{2}}\right)}{\left(P e_{b}+\frac{1}{4}\right) e^{2 P e_{b}}-P e_{b} \sqrt{-2 \pi P e_{b}} \operatorname{erfc}\left(\sqrt{-2 P e_{b}}\right)}
\end{aligned}
$$

The steady-state solution for no hydrodynamics when $-P e_{b} \gg 1$ is given by

$$
f_{N H}(r)=-\frac{4}{3} \frac{e^{\frac{1}{2} P e_{b}\left(r^{2}-4\right)}}{(r / 2)^{3}}
$$

The results obtained from a numerical solution of (2.27) were found to be consistent with the analytical results given above in the respective limits. See Appendix A at the end of this chapter for the detailed solution of the Smoluchowski equation with no hydrodynamic interactions.

In the case of positive expansion $\left(P e_{b}>0\right)$, a steady-state solution could not be found: the expansion flow along with the Brownian and interparticle forces causes the particles to move apart and there is no steady microstructure in which these forces balance. The particles continue to move apart forever at a rate proportional to $P e_{b} r$, and $g(r, t) \rightarrow 0$ around any given particle. However at separations of $r \gg e^{P e_{b} t}$ the microstructure is unchanged because the disturbance has not propagated that far and $g(r, t) \rightarrow 1$ in this outer region, thus satisfying the boundary condition at $r \rightarrow \infty$. Again, the overall number density decreases with time as prescribed by the expansion flow.

The unsteady equation (2.26) for the perturbed microstructure can be solved numerically and it was found that $g(r, t) \rightarrow 0$ in an ever increasing part of the solution domain as $t \rightarrow \infty$, as expected. At long times, the solution profiles for $f(r)$ can be collapsed onto a single curve by scaling the separation as $r / e^{P e_{b} t}$. For the case of no hydrodynamic interactions the equation can be simplified and solved analytically via a Laplace transform in time. The results obtained thus were consistent with the unsteady numerical solution. It was found that for large positive Péclet numbers, as well as at large times for any $P e_{b}>0$, the pair-distribution function at contact decreases exponentially as $g(2, t) \sim e^{-4 P e_{b}^{2} t}$. The implications of a continually expanding microstructure for the $O\left(\phi^{2}\right)$ contribution to bulk viscosity are not known. As a result, the current study focuses on negative expansion rates - compression - only and the bulk viscosity is computed for all negative Péclet 
numbers.

\subsubsection{Hydrodynamic interactions}

In order to obtain a complete solution for the pair-distribution function with hydrodynamics, we need to know $v^{\prime}(r \hat{b})$, the relative velocity between two force-and torque-free particles in an expansion flow. The motion of the particles is influenced by the hydrodynamic interactions between them. The hydrodynamic interactions between two particles in Stokes flow can be expressed in the form of a grand resistance matrix, relating the hydrodynamic force and stresslet to the velocity and rate-of-strain according to

$$
\left(\begin{array}{c}
\boldsymbol{F}^{H} \\
\boldsymbol{S}^{H}
\end{array}\right)=-\left(\begin{array}{cc}
\boldsymbol{R}_{F U} & \boldsymbol{R}_{F E} \\
\boldsymbol{R}_{S U} & \boldsymbol{R}_{S E}
\end{array}\right) \cdot\left(\begin{array}{c}
\boldsymbol{U}-\boldsymbol{U}^{\infty} \\
-\boldsymbol{E}^{\infty}
\end{array}\right),
$$

where the superscript $\infty$ denotes the imposed flow. Traditionally [Kim and Karrila 1991], only traceless stresslets were considered. However, there is no such restriction on the stresslets or the rate-of-strain. The pressure moment, defined as the trace of the particle stresslet was calculated by Jeffrey et al. [1993] for two particles in an incompressible (traceless) Stokes flow. Specifically, the pressure moment is related to the particle velocities and their rate-of-strain as

$$
\left(\begin{array}{c}
S_{1}^{H} \\
S_{2}^{H}
\end{array}\right)=-\eta\left(\begin{array}{llll}
\boldsymbol{P}_{11} & \boldsymbol{P}_{12} & \boldsymbol{Q}_{11} & \boldsymbol{Q}_{12} \\
\boldsymbol{P}_{21} & \boldsymbol{P}_{22} & \boldsymbol{Q}_{21} & \boldsymbol{Q}_{22}
\end{array}\right) \cdot\left(\begin{array}{c}
\boldsymbol{U}_{1}-\boldsymbol{U}\left(\boldsymbol{x}_{1}\right) \\
\boldsymbol{U}_{2}-\boldsymbol{U}\left(\boldsymbol{x}_{2}\right) \\
\boldsymbol{E}_{1}-\boldsymbol{E}_{\infty} \\
\boldsymbol{E}_{2}-\boldsymbol{E}_{\infty}
\end{array}\right),
$$

where $\boldsymbol{P}_{\alpha \beta}=\pi\left(a_{\alpha}-a_{\beta}\right)^{2} X_{\alpha \beta}^{P} \boldsymbol{d}$ relates the pressure moment to the particle velocities. The $X_{\alpha \beta}^{P}$ are hydrodynamic resistance functions and $\boldsymbol{d}=\left(\boldsymbol{x}_{2}-\boldsymbol{x}_{1}\right) /\left|\boldsymbol{x}_{2}-\boldsymbol{x}_{1}\right|$. The analogous expression for the tensors relating the pressure moment to an imposed linear traceless shear flow was given as $\boldsymbol{Q}_{\alpha \beta}=\pi\left(a_{\alpha}-a_{\beta}\right)^{3} X_{\alpha \beta}^{Q}\left[\boldsymbol{d} \boldsymbol{d}-\frac{1}{3} \boldsymbol{I}\right]$, where the $X_{\alpha \beta}^{Q}$ are also hydrodynamic resistance functions. From (2.30) and (2.31) and $\boldsymbol{R}_{F E}^{\dagger}=\boldsymbol{R}_{S U}$ the relative velocity between two force- and torque-free particles 
in an expansion flow was found to be

$$
v^{\prime}(r \hat{b})=-\frac{4}{3} \frac{1}{\hat{b}} G(r \hat{b})\left(X_{11}^{P}-X_{12}^{P}\right),
$$

where the hydrodynamic functions are evaluated at $r \hat{b}$.

During the course of this study, it became apparent that linear compressible flows (with a nonzero trace) should also be considered for calculation of the pressure moments. When the imposed flow has a nonzero trace, there is an additional term in the resistance matrices. This term was designated as $T_{\alpha \beta}^{Q}$, so that the complete $\boldsymbol{Q}_{\alpha \beta}$ function becomes

$$
\hat{\boldsymbol{Q}}_{\alpha \beta}=\pi\left(a_{\alpha}-a_{\beta}\right)^{3}\left[X_{\alpha \beta}^{Q}\left(\boldsymbol{d} \boldsymbol{d}-\frac{1}{3} \boldsymbol{I}\right)+T_{\alpha \beta}^{Q} \frac{1}{3} \boldsymbol{I}\right]
$$

such that the trace of $\hat{\boldsymbol{Q}}_{\alpha \beta}$ is no longer zero by design. The scalar functions $T_{\alpha \beta}^{Q}$ were determined for all separations and particles of different radii [Khair et al. 2006]. A lubrication theory expression was derived for very small separations and multipole expansion was used for all other separations following the method of Jeffrey and Onishi [1984]. A plot of $T_{11}^{Q}$ and $T_{12}^{Q}$ against the dimensionless separation distance $s$ is shown in Figure 2.1 for equal sized spheres. The two particle contribution to

the function is singular near contact scaling as $T_{\alpha \beta}^{Q} \sim \xi^{-1}$, where $\xi=s-2$ and decays with scaling $T_{11}^{Q} \sim s^{-4}$ and $T_{12}^{Q} \sim s^{-5}$ as $s \rightarrow \infty$.

The hydrodynamic functions described here were used to determine the particle stresslets resulting from Brownian, interparticle-force and rate-of-strain interactions between particles and the result was used to calculate the corresponding contributions to the effective bulk viscosity of the suspension.

\subsubsection{Expressions for the bulk viscosity}

The $O\left(\phi^{2}\right)$ contribution to particle stresslets arises from hydrodynamic interactions between particles, which can be expressed in terms of known hydrodynamic functions as done in Brady et al. [2006]. The particle stresslets (2.6) are related linearly to the imposed flow conditions via a grand resistance matrix, the elements of which contain the hydrodynamic resistance functions [Kim and 
Karrila [1991]]. For particle pairs the stresslets due to expansion flow can be simplified to give:

$$
\begin{aligned}
& S^{B}(r)=\frac{2}{3} \frac{k T}{\hat{b}}\left[\frac{1}{r}\left(X_{12}^{P} x_{21}^{a}+X_{11}^{P} x_{11}^{a}\right)+\frac{\partial}{\partial r}\left(X_{12}^{P} x_{21}^{a}+X_{11}^{P} x_{11}^{a}\right)\right] \\
& S^{P}(r)=\frac{1}{2} k T \delta(r-2)\left[r+v^{\prime}(r \hat{b})\right] \\
& S^{E}(r)=\frac{8}{3} \pi a^{3} \eta S\left[\left(T_{12}^{Q}+T_{22}^{Q}\right)-\frac{1}{4} \hat{b}\left(X_{12}^{P}+X_{22}^{P}\right) v^{\prime}(r)\right] .
\end{aligned}
$$

The stresslets for particle-pair interactions were computed as a function of the interparticle distance and then averaged over the particle microstructure using the pair-distribution function to get the $O\left(\phi^{2}\right)$ contribution to the bulk stress.

The deviation of the trace of the bulk stress from its equilibrium value divided by the average rate of expansion gives the bulk viscosity of the suspension, which to second order in volume-fraction of particles can be written as

$$
\kappa_{e f f}=\left(\kappa+\frac{4}{3} \eta \phi\right) \frac{1}{1-\phi}+\left(\kappa^{B}+\kappa^{P}+\kappa^{E}\right) \phi_{b}^{2}
$$

where the three $O\left(\phi_{b}^{2}\right)$ coefficients correspond to the Brownian, interparticle-force and rate-of-strain contributions to the bulk viscosity. It should be noted that the volume fraction for the two-particle contributions is based on the excluded-volume radius $b$ of the particles. The explicit forms for these contributions are given by

$$
\begin{aligned}
\kappa^{B} & =-\eta \frac{1}{\hat{b}^{2}} \frac{1}{2} \int_{2}^{\infty}\left[\frac{\mathrm{d}}{\mathrm{d} r}\left[X_{22}^{P} x_{22}^{a}+X_{21}^{P} x_{21}^{a}\right]+\frac{2}{r}\left[X_{22}^{P} x_{22}^{a}+X_{21}^{P} x_{21}^{a}\right] \|_{r \hat{b}} f(r) r^{2} \mathrm{~d} r,\right. \\
\kappa^{P} & =-\eta \frac{1}{\hat{b}} \frac{3}{2} v(2 \hat{b}) f(2), \\
\kappa^{E} & =\eta \frac{1}{\hat{b}^{3}} 2 \int_{2}^{\infty}\left[\left(T_{12}^{Q}+T_{22}^{Q}\right)^{\prime}-\frac{1}{4} \hat{b}\left(X_{22}^{P}-X_{21}^{P}\right) v^{\prime}(r \hat{b})\right]\left(1+P e_{b} f(r)\right) r^{2} \mathrm{~d} r,
\end{aligned}
$$

where $x_{\alpha \beta}^{a}$ are the non-dimensional scalar mobility functions relating velocity to force [Kim and Karrila 1991]. All the hydrodynamic functions are evaluated at $r \hat{b}$ and the prime on $\left(T_{12}^{Q}+T_{22}^{Q}\right)$ is a reminder that the isolated particle value has been removed. All the two-particle contributions are positive and are proportional to the shear viscosity $\eta$ as they arise from the incompressible disturbance flow caused by the presence of the rigid particles. 


\subsection{Results and discussion}

\subsubsection{Numerical solution of the perturbed microstructure}

The complete steady-state pair-distribution equation (2.27) was solved numerically using a finite difference method for a wide range of negative Péclet numbers. The resulting solution for $f(r)$ typically has a steep slope near contact $(r=2)$ and decays as $r \rightarrow \infty$. The far boundary was chosen at a large value of $r$ such that the solution is expected to be close to round-off error in that region and $f$ was set to 0 at the far end. The location of the far boundary was varied until the solution became independent of its position. The finite difference grid was discretized with a geometrically increasing number of points closer to contact where the slope of $f$ is expected to be steep, resulting in a more accurate solution for $f$.

When the Péclet number is large, a boundary layer develops and the radial dimension has to be scaled in order to get an accurate numerical solution. The thickness of the boundary layer is also influenced by the strength of the hydrodynamic interactions. The following scaling was used for the boundary-layer coordinate:

$$
Y=\frac{r \beta-2}{\epsilon},
$$

where $\epsilon=-1 / P e_{b}$ and $\beta$ is given by

$$
\beta= \begin{cases}\hat{b} & \text { if } \hat{b}-1 \ll \epsilon \\ 1+\epsilon(\hat{b}-1) & \text { if } \hat{b}-1 \sim \epsilon \text { and } \hat{b}-1 \ll 1 \\ 1+\epsilon^{3 / 2} & \text { otherwise }\end{cases}
$$

The $Y$ coordinate was further transformed as $z=\ln Y$ for the numerical solution, in order to expand the boundary layer further. Inside the boundary layer, the hydrodynamic functions present in (2.27) are strong functions of the interparticle distance $\xi=r \hat{b}-2$ when hydrodynamic interactions are important $(\hat{b} \rightarrow 1)$. Therefore the relevant scaling is obtained by expanding $\xi$ with the small parameter $\epsilon$. However, when hydrodynamic interactions are not important, boundary-layer effects become more dominant and $r-2$ is scaled instead with $\epsilon$ to get the scaled coordinate. In either case, the relevant small parameter had to be added to the scaling in order to avoid having log of zero at contact. In the case of nearly full hydrodynamic interactions, the appropriate small parameter is 
$\epsilon(\hat{b}-1)$ and for weaker hydrodynamic interactions, a small parameter of $\epsilon^{3 / 2}$ was found to give good results. These considerations led to the expressions for $\beta$ defined in (2.39).

Second-order finite differences on the non-linear grid were employed to discretize the derivative terms in (2.27). The finite difference matrices obtained for the steady-state equation were tridiagonal and were inverted using Gaussian elimination to obtain the solution. The unsteady equation (2.26) was also solved numerically to explore the solution when $P e_{b}>0$. The "Method of Lines" [Schiesser 1991], where the spatial part of the equation is discretized resulting in ordinary differential equations in time at each grid point, was used. The resulting set of equations was integrated in time using widely available stiff ODE solvers [Shampine and Reichelt 1997].

Figure 2.2 shows $f(r)$, the perturbation to the microstructure scaled by the Péclet number, for small $P e_{b}\left(=-10^{-5}\right)$ for the two limiting cases of no hydrodynamic interactions: $\hat{b} \rightarrow \infty$ and nearly pure hydrodynamic interactions: $\hat{b}=1.00001$. The curves are the same as those obtained for the linear response regime solution (3.33) because higher-order terms are negligible for small values of $P e_{b}$. The two curves are very similar and both decay as $1 / r$ for large $r$. For intermediate values of $\hat{b}, f(r)$ lies between these two curves.

A boundary layer is formed when $\left|P e_{b}\right| \gg 1$. The perturbation of the microstructure takes place inside this boundary layer and so, $f(r)$ decays very rapidly within a very short distance of the order of the boundary layer thickness $\sim O\left(1 /\left|P e_{b}\right|\right)$. Figure 2.3 shows $f(r)$ for the case of no hydrodynamic interactions: $\hat{b} \rightarrow \infty$, and nearly full hydrodynamic interactions: $\hat{b}=1.00001$ for large $P e_{b}\left(=-10^{3}\right)$. Both the curves decay much faster than the curves for low $P e_{b}$ and their values at contact are also smaller. In the case of no hydrodynamic interactions $f(r)$ decays exponentially at large distances as can be seen from (2.29), while in the case of nearly full hydrodynamics, $f(r)$ decays as $1 / r$ away from contact.

The contact value of $f(r)$ (at $r=2$ ) is shown in Figure 2.4 as a function of the Péclet number for varying levels of hydrodynamic interactions depending on the value of $\hat{b}$. As $P e_{b}$ decreases, $f(2)$ converges to the linear-response solution. For large values of $P e_{b}, f(2)$ approaches a different limiting value depending on $\hat{b}$. In the case of no hydrodynamics, the limiting value can be verified to be $f(2)=-\frac{4}{3}$ (by simplifying (2.29) for large $P e_{b}$ ). The limiting value for the case of full hydrodynamics, $\hat{b} \equiv 1$ was numerically calculated to be $f(2) \simeq-2.75$. It can be seen from this figure that $f(r)$ decreases as the Péclet number becomes larger for all $\hat{b}$ and eventually reaches a 
constant limiting value, which suggests that a 'thinning' of the averaged properties of the suspension might take place as $P e_{b} \rightarrow-\infty$.

\subsubsection{The $O\left(\phi_{b}^{2}\right)$ bulk viscosity coefficients}

The two-particle contributions to the bulk viscosity due to Brownian, interparticle-force and rateof-strain interaction are shown as a function of the ratio of the excluded-volume to actual particle radius $\hat{b}=b / a$ in Figures 2.5, 2.6 and 2.7, respectively, for different values of $P e_{b}$. For small $P e_{b}$ the curves obtained are the same as those for the linear-response regime.

As $\hat{b} \rightarrow 1$, the Brownian and rate-of-strain contributions reach a finite limiting value, while the interparticle-force contribution vanishes (as $(\hat{b}-1)$ ) because the relative particle velocity is zero at contact $(r=2)$. The limiting values of the $O\left(\phi_{b}^{2}\right)$ coefficients for full hydrodynamics, $\hat{b} \equiv 1$ as $P e_{b} \rightarrow 0$ are $\kappa^{B}=5.348 \eta$ and $\kappa^{E}=1.57 \eta$. For large Péclet numbers the Brownian contribution vanishes as well because the motion of the particles is influenced more by the flow velocity and Brownian motion becomes less important. As $P e_{b} \rightarrow-\infty$ only the rate-of-strain contribution is nonzero for full hydrodynamics with a limiting value of $\kappa^{E}=5.03 \eta$, which is also the limiting value of the total $O\left(\phi_{b}^{2}\right)$ contribution. There is also an $O\left(\phi^{2}\right)$ term in the single-particle contribution to bulk viscosity given by $\left(\kappa+\frac{4}{3} \eta\right) \hat{b}^{-6}$, which is comparable to the two-particle contribution for all Péclet numbers when $\hat{b} \rightarrow 1$. The magnitude of this term is smaller than the total two-particle contribution as long as the bulk viscosity of the fluid is not significantly greater than its shear viscosity.

As $\hat{b}$ increases the hydrodynamic interactions are reduced and the Brownian and rate-of-strain contributions decrease, while the interparticle-force contribution increases. Once $\hat{b}$ exceeds a value between 1.2 and 1.244 corresponding to the range of $P e_{b} \rightarrow-\infty$ and $P e_{b} \rightarrow 0$, respectively, the particles are kept so far apart by the excluded-volume interaction that all two-particle contributions to the bulk viscosity decrease to zero, scaling as: $\kappa^{B} \sim \hat{b}^{-7}, \kappa^{P} \sim \hat{b}^{-1}$ and $\kappa^{E} \sim \hat{b}^{-9}$ as $\hat{b} \rightarrow \infty$. The interparticle force contribution dominates over all the other terms in this limit, including the $O\left(\phi^{2}\right)$ single-particle contribution.

The total two-particle contribution to bulk viscosity as a function of the Péclet number for varying levels of hydrodynamics, depending on $\hat{b}$, is shown in Figure 2.8. For small values of $\left|P e_{b}\right|$ the value of the total contribution is close to the value obtained from the linear-response regime solution. As $\left|P e_{b}\right|$ increases the Brownian contribution decreases faster than the rate-of-strain con- 
tribution grows, resulting in a net decrease in the bulk viscosity. As $P e \rightarrow-\infty$, the Brownian contribution goes to zero scaling as $\kappa^{B} \sim\left|P e_{b}\right|^{-1}$. Once $\left|P e_{b}\right|$ exceeds approximately 0.61 , the rate-of-strain contribution becomes dominant and the total bulk viscosity grows and then plateaus, finally reaches a limiting value of $\simeq 5.03 \eta$ as $P e \rightarrow-\infty$ in the limit of full hydrodynamic interactions. Thus, a 'thickening' of the total bulk viscosity takes place as it does for the shear viscosity [Bergenholtz et al. 2002]. The thickening effect is observed only when hydrodynamic interactions are important $(\hat{b} \leq 1.2)$ and when the rate-of-strain contribution increases sufficiently to overcome the Brownian contribution for large values of $P e_{b}$. For values of $\hat{b}>1.2$, only the interparticle force contribution dominates and the other two contributions are negligibly small and, as a result, only a thinning effect is observed.

The excluded annulus model can also be used to model hard spheres with no hydrodynamic interactions by setting the actual sphere radius equal to the excluded volume radius $b$ and setting $\hat{b} \rightarrow$ 1. The Stokes-Einstein-Sutherland diffusivity of the particles in now given by $D=k T / 6 \pi \eta b$. From (3.6) only the $\kappa^{P}$ contribution is nonzero and depends only on the perturbation to the microstructure at contact given by $f(2)$. In a hard sphere system with no hydrodynamics only hard sphere collisions of the particles contribute to the stress and there is no direct Brownian contribution or hydrodynamic contribution. Particles see each other only when they touch and therefore only the pair-distribution function at contact determines the total stress. In the absence of hydrodynamics the relative particle velocity is simply $v(r \hat{b})=r$ and the $O\left(\phi^{2}\right)$ correction to the bulk viscosity is simply $\kappa^{P}=-3 f(2) \eta$. The hard-sphere bulk viscosity contribution without hydrodynamic interactions is shown in Figure 2.9 as a function of the Péclet number. In the linear-response limit $\left(P e_{b} \rightarrow 0\right)$ it asymptotes to a value of $\kappa^{P}=12 \eta$ and shear thins as $P e_{b}$ increases, eventually reaching a plateau at $\kappa^{P}=4 \eta$.

Finally, all the $O\left(\phi_{b}^{2}\right)$ contributions vanish when hydrodynamic interactions are not important $(\hat{b} \rightarrow \infty)$ because the volume fraction is based on the excluded-volume radius $b$. When $\hat{b} \gg 1$ the actual volume fraction $\phi$ is so small that the particles are essentially isolated and only the singleparticle contribution to bulk viscosity remains. The inter-particle force coefficient decays most slowly as $1 / \hat{b}$ because the particle diffusivity $D=k T / 6 \pi \eta a$ depends on the actual or hydrodynamic size of the particle. The two-particle contributions can also be evaluated based on the actual volume fraction $\phi$, in which case they scale as: $\kappa^{B} \sim \hat{b}^{-1}, \kappa^{P} \sim \hat{b}^{5}$ and $\kappa^{E} \sim \hat{b}^{-3}$ as $\hat{b} \rightarrow \infty$. The Brownian and rate-of-strain contributions still decay as $\hat{b} \rightarrow \infty$, but the inter-particle force contribution grows 
because the range of repulsive forces increases with $\hat{b}$ when the actual volume fraction is used. The corresponding curves are shown in Figure 2.10 for the case of small $P e_{b}$. The behavior for other values of $P e_{b}$ will be qualitatively the same as $\hat{b} \rightarrow \infty$.

\subsection{Concluding remarks}

The effective bulk viscosity of a suspension of particles in a fluid was defined based on the average macroscopic stress in a way analogous to the definition of bulk viscosity for a pure fluid. The bulk viscosity relates the deviation of the trace of the macroscopic stress from its equilibrium value to the average rate of expansion of the suspension. Expressions were derived for computing the bulk viscosity of a suspension undergoing uniform volume expansion for all volume fractions of particles and for all expansion rates.

In the present study, the specific problem of a suspension of rigid spherical particles in a compressible fluid undergoing uniform compression at a steady rate was considered in order to calculate the bulk viscosity of the suspension to second order in the volume fraction of particles $\phi$. However, the general formulae derived for the bulk viscosity can also be used for arbitrary time-dependent flows and should apply to bubbles and drops as well as rigid particles. The correction to bulk viscosity was found to be proportional to the shear viscosity of the fluid, implying that the effective bulk viscosity of the suspension may be significant even if the suspending fluid has a negligible bulk viscosity.

The disturbance flow due to a single particle in an expanding fluid results in the $O(\phi)$ correction to the bulk viscosity, while two-particle interactions lead to the $O\left(\phi^{2}\right)$ correction. In the case of a compressive flow, a steady-state solution for the pair-distribution function was obtained for all negative Péclet numbers and used to calculate the bulk viscosity. It was shown that in the presence of hydrodynamic interactions, a 'thinning' of the bulk viscosity takes place as the Péclet number increases in magnitude starting from a very small negative value, but, as $P e_{b} \rightarrow-\infty$, the stresslet contribution due to the rate-of-strain becomes dominant, the bulk viscosity increases again similar to a 'thickening' effect and eventually reaches a limiting value.

When the fluid is expanding $\left(P e_{b}>0\right)$, however, a steady-state distribution of particles could not be achieved. Even so, the unsteady equations can be solved numerically to obtain the evolu- 
tion of the particle microstructure in space and time. It should be noted that during the course of compression, the volume fraction of particles continually increases to close packing (or decreases in the case of expansion) and therefore, some care is needed in defining the bulk viscosity for a given volume fraction. At very high rates of compression, the time scale for the evolution of the microstructure may be the same or larger than the time scale for increase in number density. In that case the number density cannot be assumed to be a constant for determining the steady-state microstructure and therefore, a steady bulk viscosity will not exist. In most practical applications, the suspension would also undergo shear in addition to expansion, and it may be useful to consider the effects of a weak expansion rate on the microstructure in a sheared suspension and the resulting bulk viscosity. 


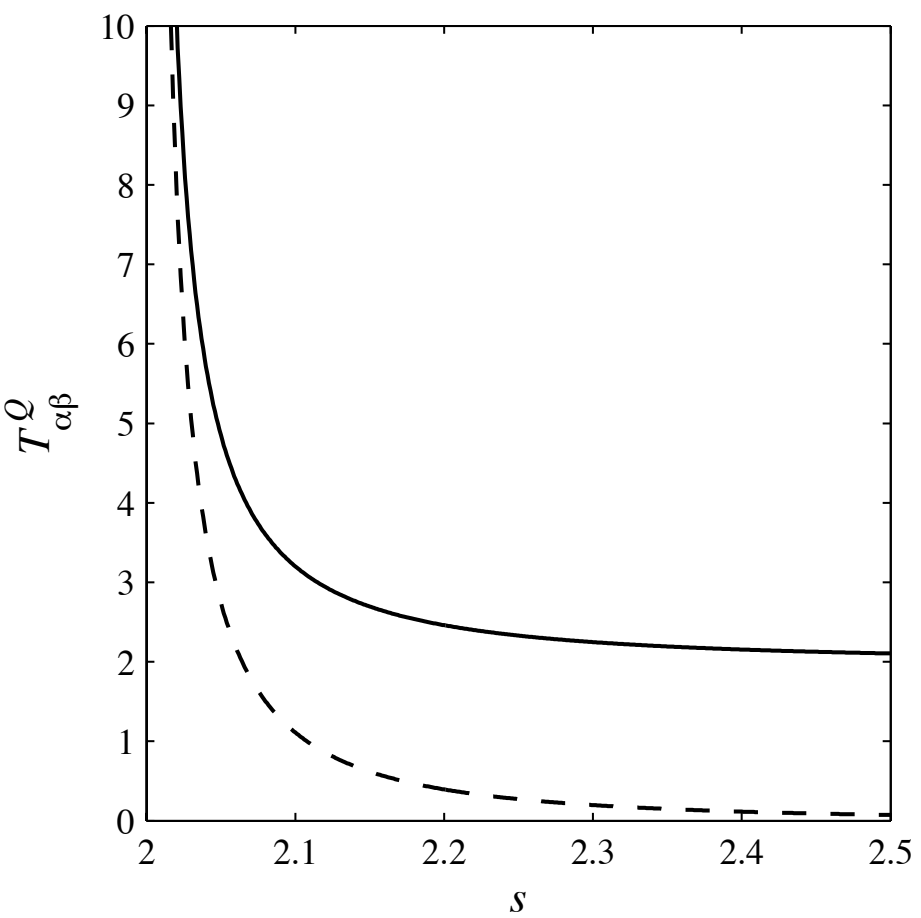

Figure 2.1: The functions $T_{11}^{Q}$ (solid line) and $T_{12}^{Q}$ (dashed line) for equal-sized spheres vs the dimensionless separation distance $s$. 


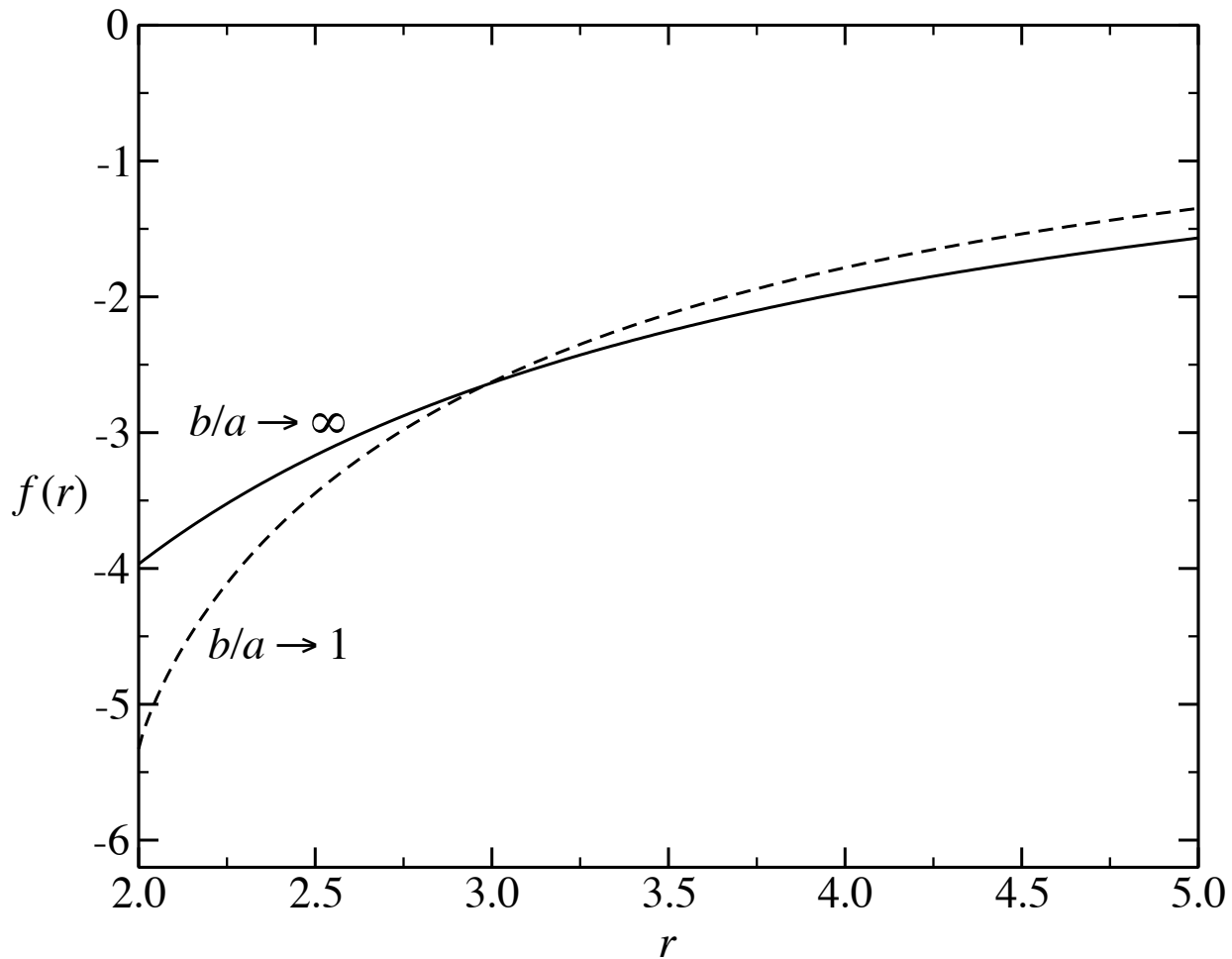

Figure 2.2: Microstructural perturbation function $f(r)$ for $b / a=1.00001$ (dashed line) and $b / a=$ 1000 (solid line) for $\left|P e_{b}\right| \ll 1$. Both curves behave as $1 / r$ for large $r$ like the linear response solution. For other values of $b / a$ the perturbation $f$ is intermediate between these two curves. 


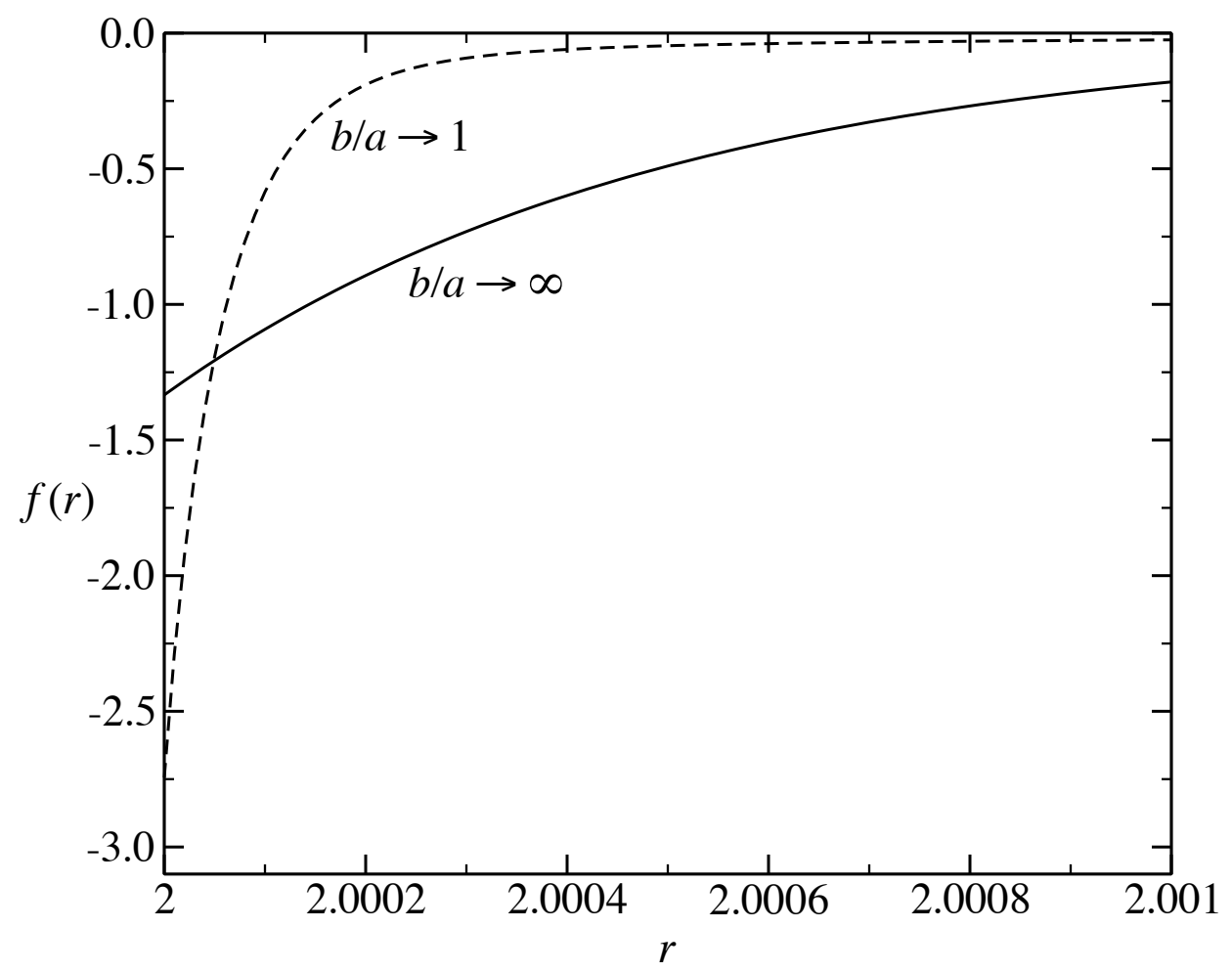

Figure 2.3: Microstructural perturbation function $f(r)$ close to the particle's surface for full hydrodynamics, $b / a=1.00001$ (dashed line) and for no hydrodynamics, $b / a=1000$ (solid line) and $P e_{b}=-10^{3}$. Both curves decay much faster than for small $P e_{b}$. For other values of $b / a$ the perturbation $f$ is intermediate between these two curves. 


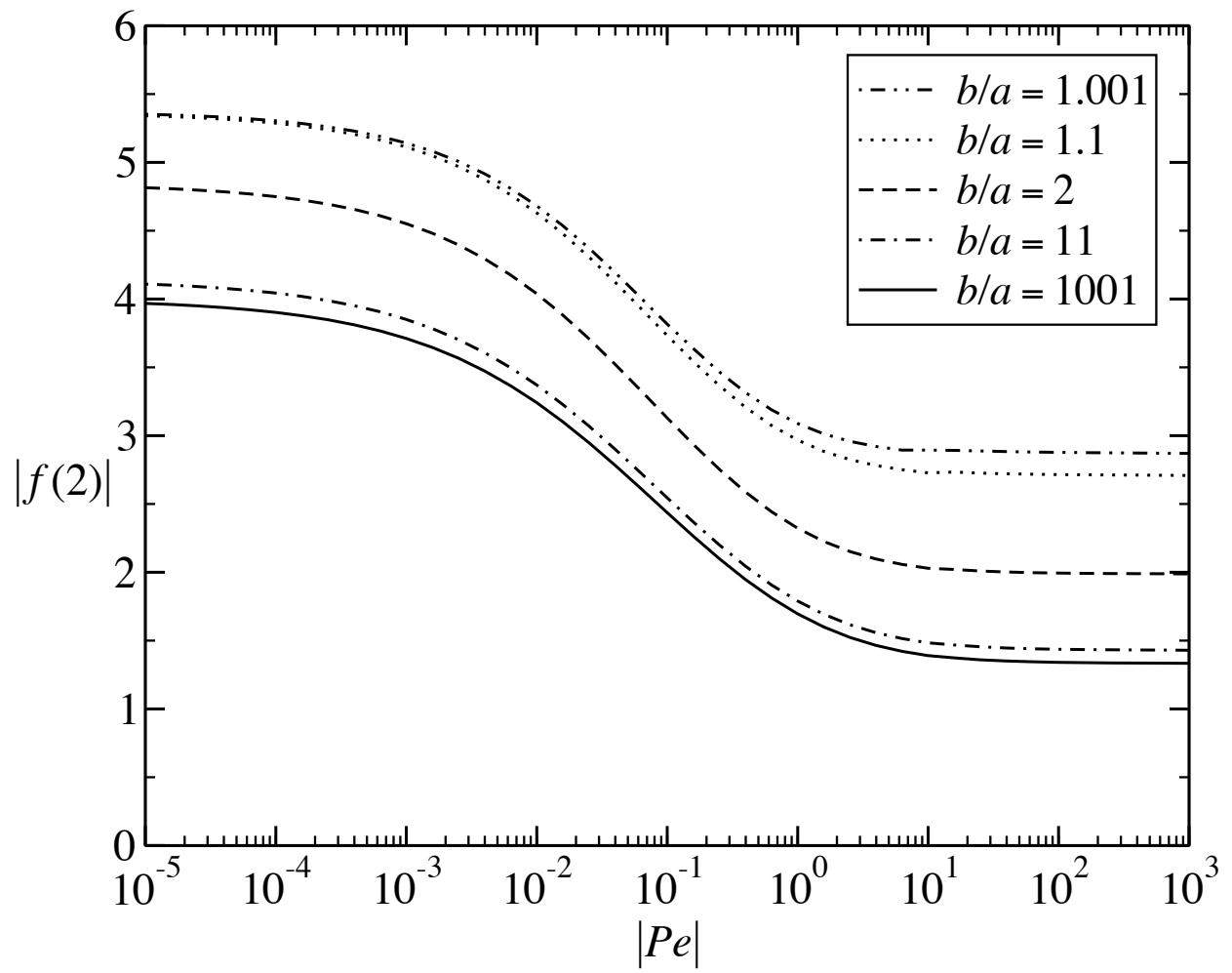

Figure 2.4: Microstructural perturbation function $f(r)$ at contact $(r=2)$ vs $P e_{b}$ for varying levels of hydrodynamic interactions. All the curves have the same behavior and exhibit a shear-thinning like behavior. The curve for no hydrodynamics corresponding to (2.29) is shown by a solid line. 


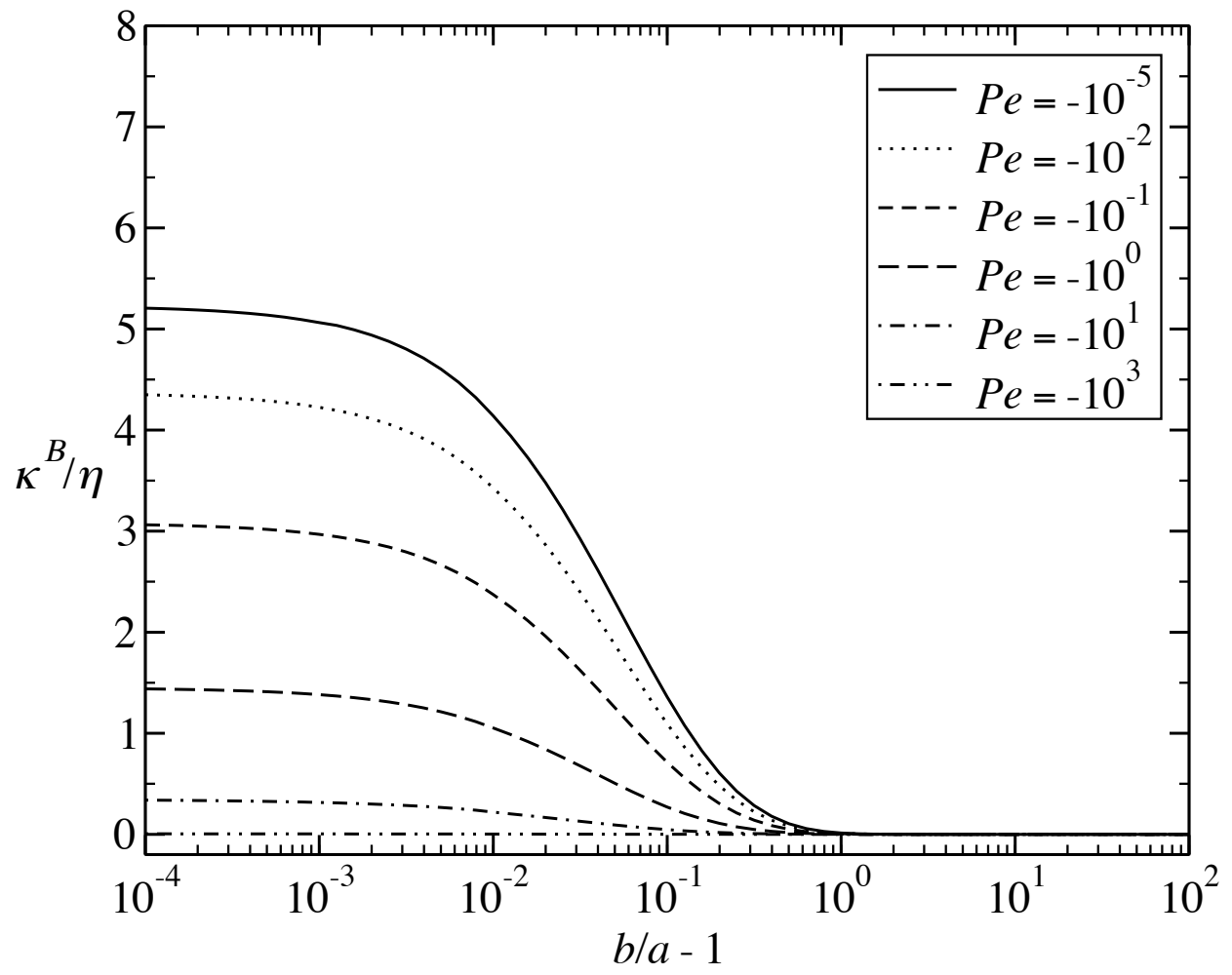

Figure 2.5: Brownian part of the $O\left(\phi_{b}^{2}\right)$ two-particle contribution to the bulk viscosity $\kappa^{B} / \eta$ vs $\hat{b}-1$, for different negative values of $P e_{b}$. 


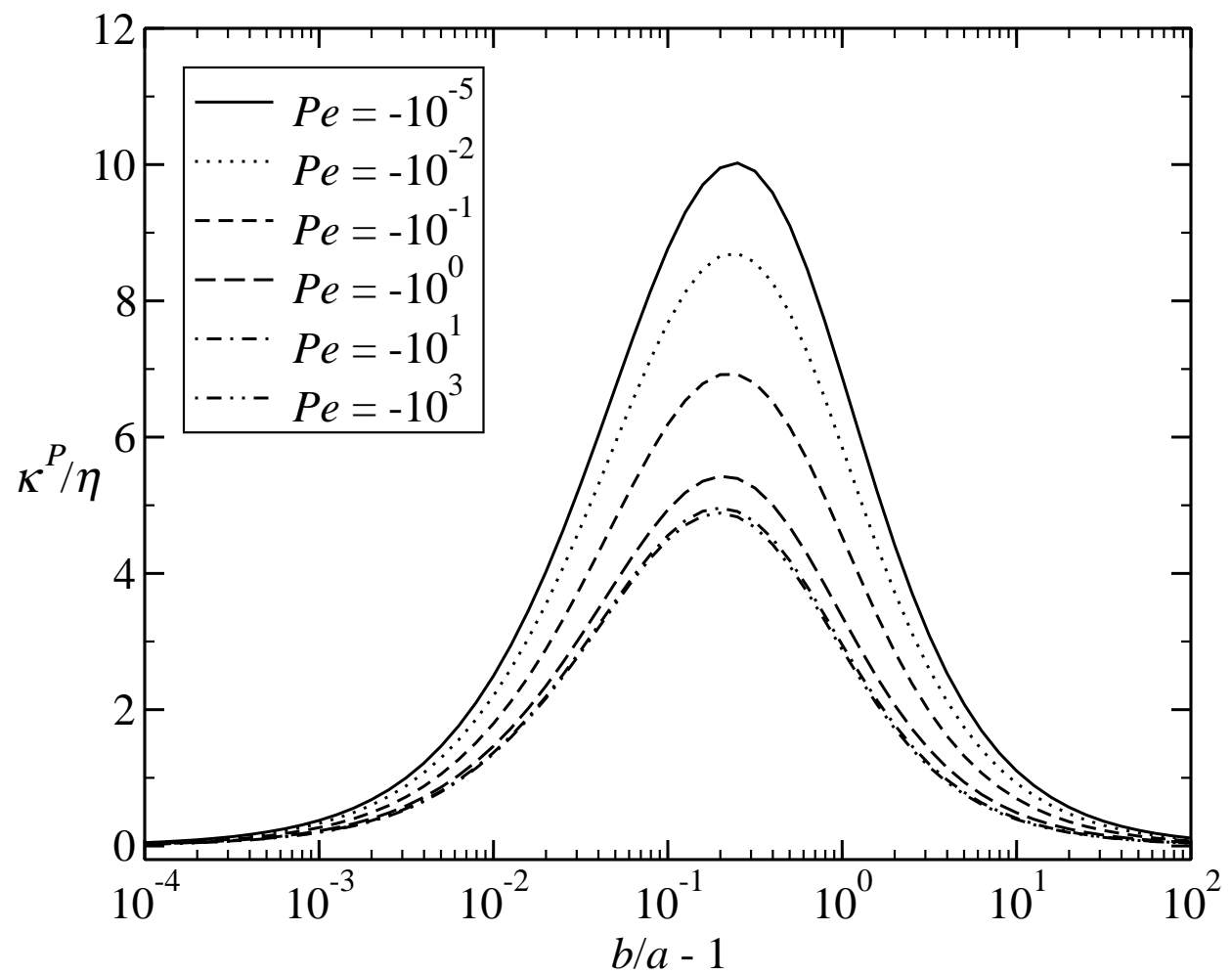

Figure 2.6: Interparticle force part of the $O\left(\phi_{b}^{2}\right)$ two-particle contribution to the bulk viscosity $\kappa^{P} / \eta$ vs $\hat{b}-1$, for different negative values of $P e_{b}$. 


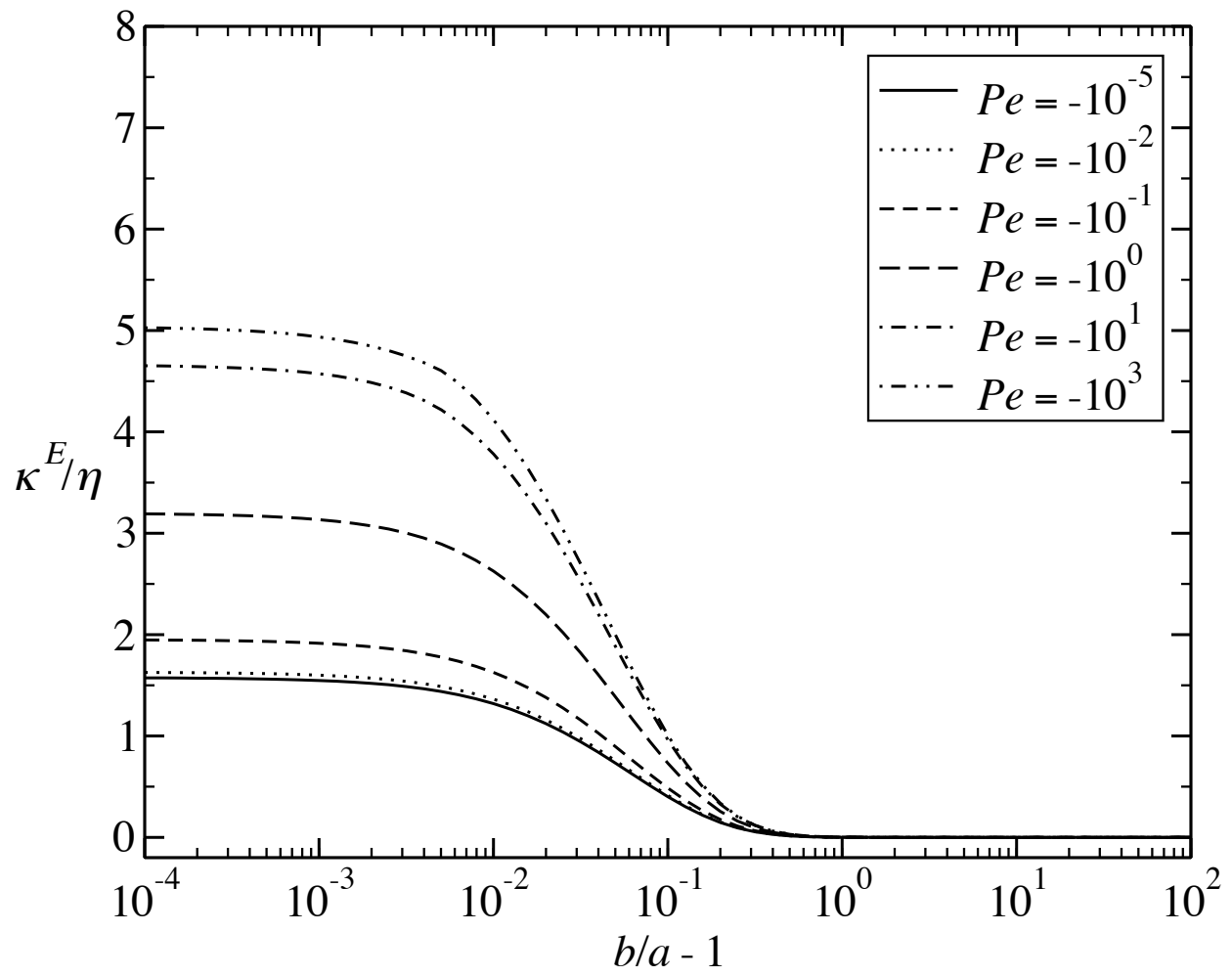

Figure 2.7: Rate-of-strain part of the $O\left(\phi_{b}^{2}\right)$ two-particle contribution to the bulk viscosity $\kappa^{E} / \eta$ vs $\hat{b}-1$, for different negative values of $P e_{b}$. 


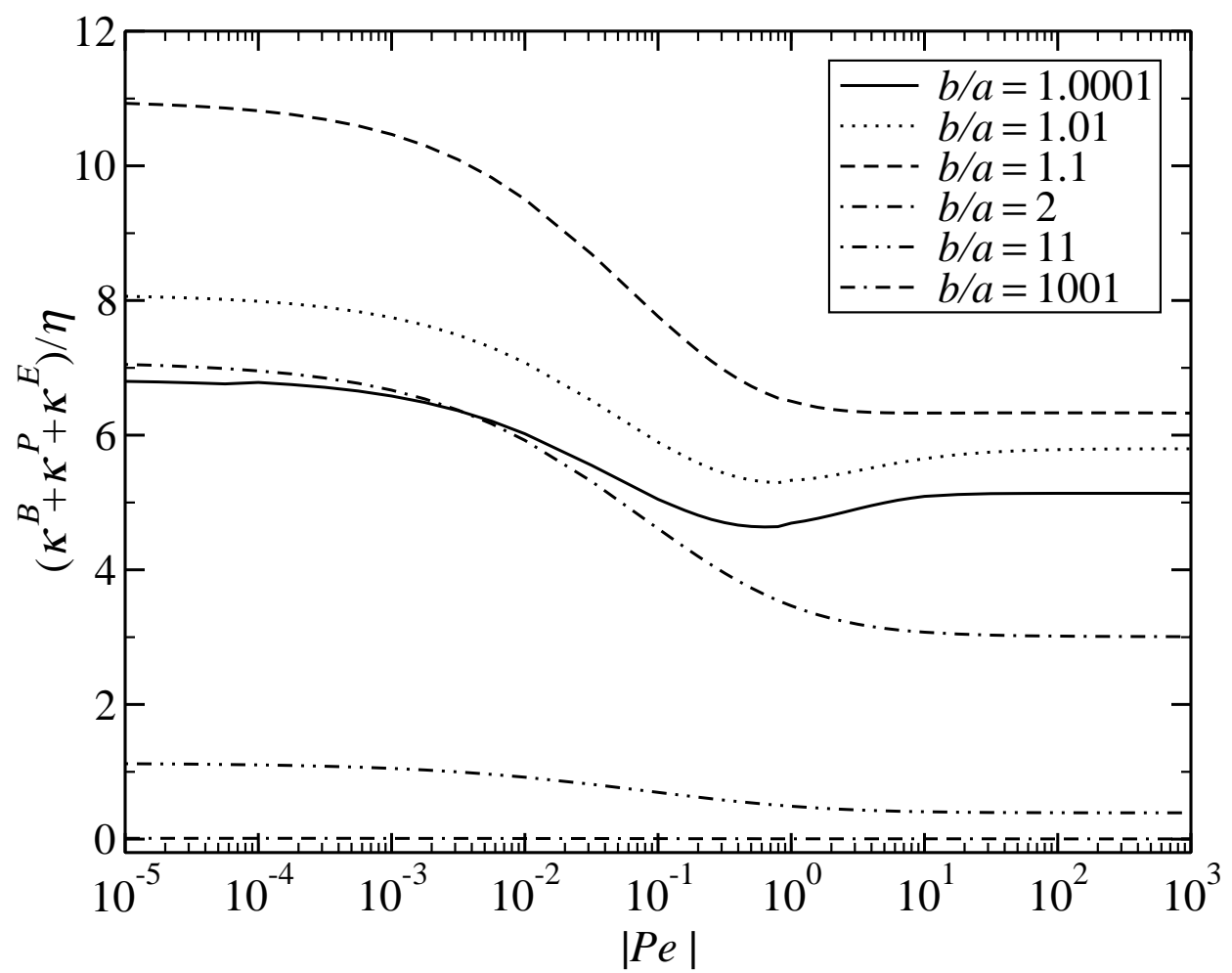

Figure 2.8: The total $O\left(\phi_{b}^{2}\right)$ two-particle contributions to the bulk viscosity $\left(\kappa^{B}+\kappa^{P}+\kappa^{E}\right) / \eta$ vs $P e_{b}$, for varying levels of hydrodynamic interaction. Shear thickening is observed as $P e_{b} \rightarrow-\infty$. As $\hat{b} \rightarrow \infty$ hydrodynamic interactions between particles decrease and the total $O\left(\phi_{b}^{2}\right)$ contribution goes to zero. 


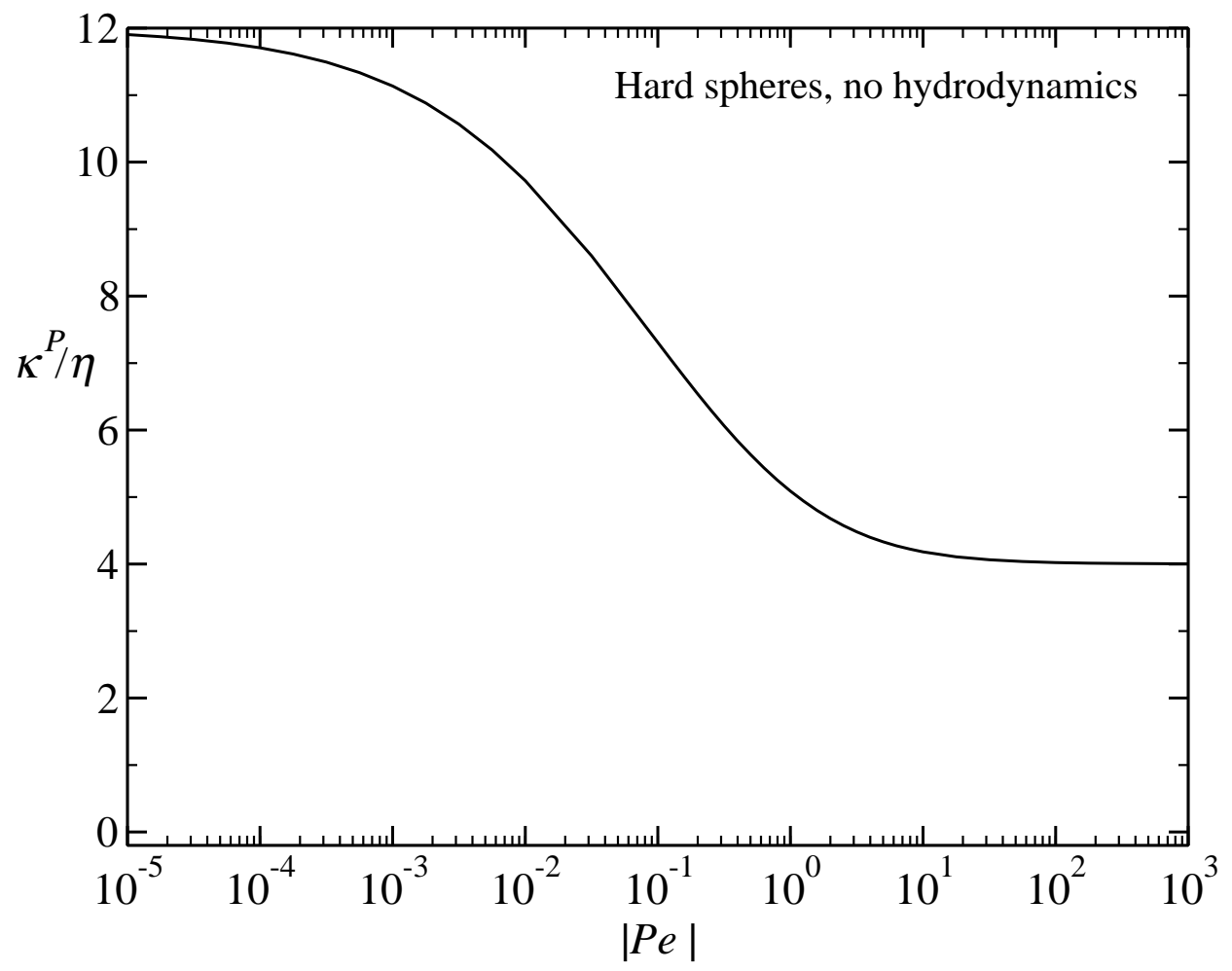

Figure 2.9: Bulk viscosity for hard spheres of radius $b$ with no hydrodynamic interactions. Only the interparticle force comprising the hard sphere potential contributes to the bulk stress. 


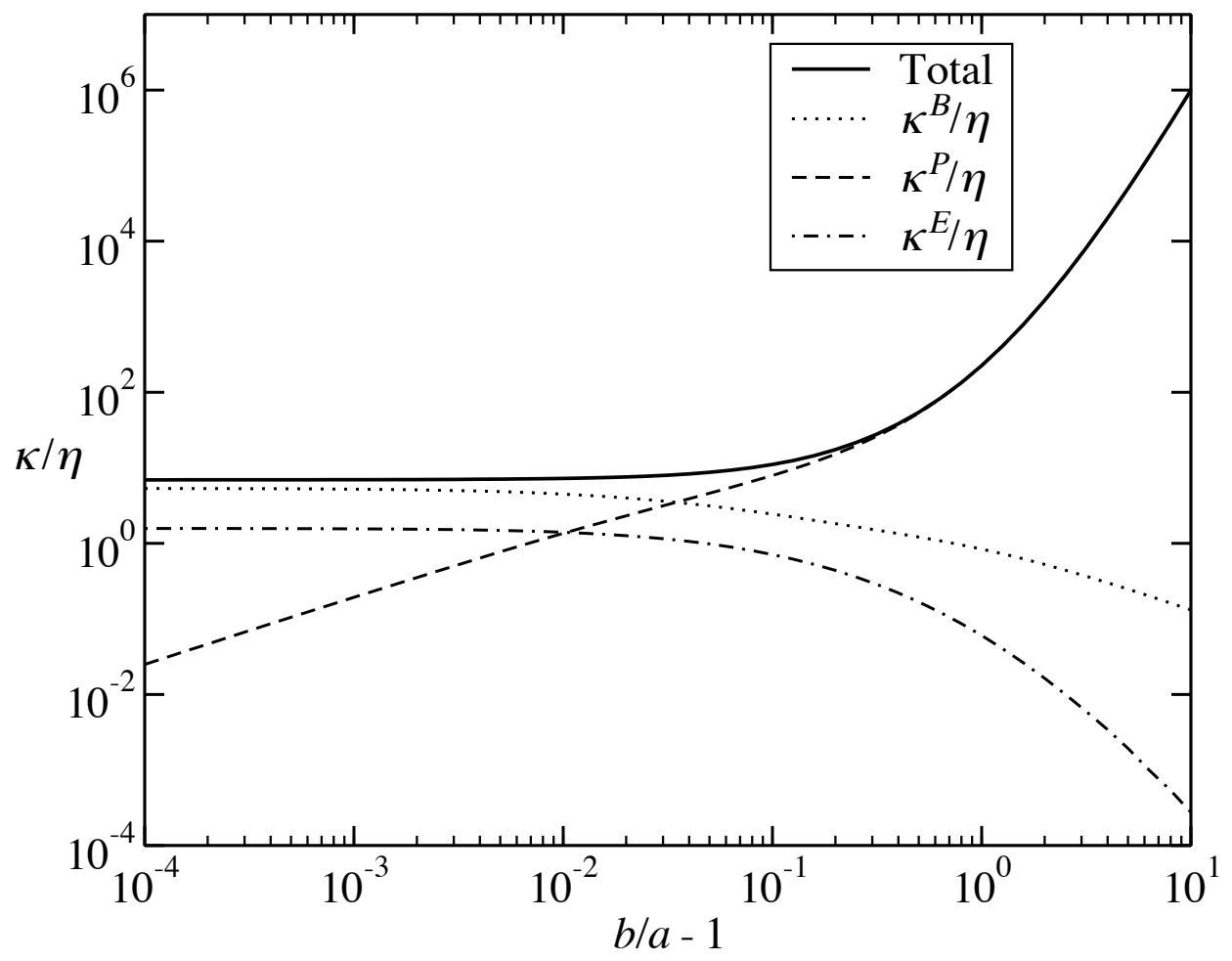

Figure 2.10: The $O\left(\phi^{2}\right)$ two-particle contributions to the bulk viscosity for small $P e_{b}$ (linear response regime) : Brownian $\kappa^{B} / \eta$ (dashed line), interparticle-force $\kappa^{P} / \eta$ (dot-dashed line), rate-ofstrain $\kappa^{E} / \eta$ (dotted line) and total $\left(\kappa^{B}+\kappa^{P}+\kappa^{E}\right) / \eta$ (solid line). Shown here are the coefficients based on the actual particle volume fraction $\phi$. 


\section{Appendix A - Smoluchowski equation with no hydrodynamics}

\section{Pair distribution function}

The pair-distribution equation is written as:

$$
\begin{aligned}
\frac{\partial g}{\partial t}+\nabla \cdot\left(\langle\mathbf{u}\rangle+\mathbf{u}^{\prime}\right) g & =\nabla \cdot \mathbf{D} \cdot \nabla g \\
g & \sim n(t) \quad \text { as } r \rightarrow \infty \\
\mathbf{n} \cdot\left(\langle\mathbf{u}\rangle+\mathbf{u}^{\prime}\right) g & =\mathbf{n} \cdot \mathbf{D} \cdot \nabla g \quad @ r=2 a \\
g & =g_{0} \quad @ t=0
\end{aligned}
$$

Let $g(\mathbf{r}, t)=n(t)[1+f(r, t)]$. Now the pair distribution equation can be written as

$$
\begin{aligned}
\dot{n}(1+f)+n \dot{f}+\nabla \cdot\left(\langle\mathbf{u}\rangle+\mathbf{u}^{\prime}\right)[n(1+f)] & =\nabla \cdot \mathbf{D} \cdot \nabla[n(1+f)] \\
\ln n(1+f)+\dot{f}+\nabla \cdot\left(\langle\mathbf{u}\rangle+\mathbf{u}^{\prime}\right)(1+f) & =\nabla \cdot \mathbf{D} \cdot \nabla(1+f) \\
-S(1+f)+\dot{f}+\nabla \cdot\left(\langle\mathbf{u}\rangle+\mathbf{u}^{\prime}\right) f+\nabla \cdot\langle\mathbf{u}\rangle+\nabla \cdot \mathbf{u}^{\prime} & =\nabla \cdot \mathbf{D} \cdot \nabla f \\
\dot{f}+\langle\mathbf{u}\rangle \cdot \nabla f+\nabla \cdot\left(\mathbf{u}^{\prime} f\right)+\nabla \cdot \mathbf{u}^{\prime} & =\nabla \cdot \mathbf{D} \cdot \nabla f \\
f & \sim 0 \quad \text { as } r \rightarrow \infty \\
\mathbf{n} \cdot\left(\langle\mathbf{u}\rangle+\mathbf{u}^{\prime}\right) f-\mathbf{n} \cdot \mathbf{D} \cdot \nabla f+\mathbf{n} \cdot\left(\langle\mathbf{u}\rangle+\mathbf{u}^{\prime}\right) & =0 \quad \text { @ } r=2 a .
\end{aligned}
$$

With no hydrodynamic interactions, $\mathbf{u}=0$ and $\mathbf{D}=2 D \mathbf{I}$.

$$
\begin{aligned}
\dot{f}+\langle\mathbf{u}\rangle \cdot \nabla f & =2 D \nabla^{2} f \\
f & \sim 0 \quad \text { as } r \rightarrow \infty \\
\mathbf{n} \cdot\langle\mathbf{u}\rangle f-2 D \mathbf{n} \cdot \nabla f & =-\mathbf{n} \cdot\langle\mathbf{u}\rangle \quad @ r=2 a .
\end{aligned}
$$


Using $\langle\mathbf{u}\rangle \cdot \nabla f=\frac{1}{3} S r \frac{\partial f}{\partial r}$, we get

$$
\begin{aligned}
\frac{\partial f}{\partial t}+\frac{1}{3} S r \frac{\partial f}{\partial r} & =2 D \frac{1}{r^{2}} \frac{\partial}{\partial r}\left(r^{2} \frac{\partial f}{\partial r}\right) \\
f & \sim 0 \quad \text { as } r \rightarrow \infty \\
2 a \frac{1}{3} S f-2 D \frac{\partial f}{\partial r} & =-2 a \frac{1}{3} S \quad @ r=2 a .
\end{aligned}
$$

Scale $r \sim \mathrm{O}(a)$ and $t \sim \mathrm{O}\left(\frac{a^{2}}{2 D}\right)$. Define $\mathrm{Pe}=\frac{1}{3} S a^{2} / 2 D$. We get

$$
\begin{aligned}
\frac{\partial f}{\partial t}+\operatorname{Pe} r \frac{\partial f}{\partial r} & =\frac{1}{r^{2}} \frac{\partial}{\partial r}\left(r^{2} \frac{\partial f}{\partial r}\right) \\
\text { or } \frac{\partial^{2} f}{\partial r^{2}}+\frac{2}{r} \frac{\partial f}{\partial r}-\operatorname{Pe} r \frac{\partial f}{\partial r}-\frac{\partial f}{\partial t} & =0 \\
f & \sim 0 \quad \text { as } r \rightarrow \infty \\
\frac{\partial f}{\partial r}-2 \operatorname{Pe} f & =2 \operatorname{Pe} \quad @ r=2
\end{aligned}
$$

\section{Solution for $\mathrm{Pe}>0$}

The pair distribution equation (A-5a) can be made independent of the Péclet number by scaling the distance and time in the following way:

$$
\text { Let } z=r \sqrt{\mathrm{Pe}} \text { and } \tau=t \mathrm{Pe} \text {. }
$$

Using the above scaling, we get

$$
\begin{aligned}
\frac{\partial^{2} f}{\partial z^{2}}+\frac{2}{z} \frac{\partial f}{\partial z}-z \frac{\partial f}{\partial z}-\frac{\partial f}{\partial \tau} & =0 \\
\frac{1}{z}\left(z \frac{\partial^{2} f}{\partial z^{2}}+2 \frac{\partial f}{\partial z}\right)-\left(z \frac{\partial f}{\partial z}+f\right)+f-\frac{\partial f}{\partial \tau} & =0 \\
\frac{\partial^{2}(z f)}{\partial z^{2}}-z \frac{\partial(z f)}{\partial z}+z f-z \frac{\partial f}{\partial \tau} & =0
\end{aligned}
$$

Let $y=z f$,

$$
\text { Let } y=z f \frac{\partial^{2} y}{\partial z^{2}}-z \frac{\partial y}{\partial z}+y-\frac{\partial y}{\partial \tau}=0
$$


The boundary conditions become

$$
\begin{aligned}
y & \sim 0 \quad \text { as } z \rightarrow \infty \\
\frac{1}{z} \frac{\partial y}{\partial z}-\frac{f}{z}-2 \sqrt{\mathrm{Pe}} f & =2 \sqrt{\mathrm{Pe}} \quad @ z=2 \sqrt{\mathrm{Pe}} \\
\Rightarrow \frac{\partial y}{\partial z}-\left(\frac{1}{2 \sqrt{\mathrm{Pe}}}+2 \sqrt{\mathrm{Pe}}\right) y & =4 \mathrm{Pe} \quad @ z=2 \sqrt{\mathrm{Pe}}
\end{aligned}
$$

The above equation can be solved by carrying out a Laplace transform in the time domain.

$$
\begin{gathered}
y(z, \tau) \rightarrow Y(z, s)=r \sqrt{\operatorname{Pe}} F(r, s) \\
\frac{\partial y}{\partial \tau}=s Y(z, s)-y(z, 0)
\end{gathered}
$$

Initial condition : $y(z, 0)=z f(z, 0)=0$

A Laplace transform of (A-7) gives

$$
\begin{aligned}
& \frac{\partial^{2} Y}{\partial z^{2}}-z \frac{\partial Y}{\partial z}+(1-s) Y=0 \\
& \text { Let } x=\frac{z}{\sqrt{2}}=r \sqrt{\frac{P e}{2}} \\
& \frac{\partial^{2} Y}{\partial x^{2}}-2 x \frac{\partial Y}{\partial x}+2 v Y=0 \\
& \text { where } v=1-s .
\end{aligned}
$$

The above equation (A-9) is a Hermite differential equation. The general solution of this equation is given by

$$
Y=A H_{v}(x)+B H_{v}(-x)
$$

where $H_{v}(z)$ is a Hermite function of order $v$. If $v=0,1,2 \ldots$ then $B=0$. As $z \rightarrow \infty,\left|H_{v}(-x)\right| \rightarrow$ $\infty[$ Lebedev 1972]. Hence, using the boundary condition at $z \rightarrow \infty, B=0$ and we get

$$
\begin{aligned}
Y & =z F=A H_{1-s}(x) \\
\text { or } F(r, s) & =\frac{A H_{1-s}\left(r \sqrt{\frac{\mathrm{Pe}}{2}}\right)}{r \sqrt{\mathrm{Pe}}}
\end{aligned}
$$


A Laplace transform of the boundary condition at $r=2$ results in

$$
\frac{\partial F}{\partial r}-2 \mathrm{Pe} F=\frac{2 \mathrm{Pe}}{s} \quad @ r=2
$$

Using $H_{v}^{\prime}(z)=2 v H_{v-1}(z)[$ Lebedev 1972], we get

$$
A=\frac{2 \sqrt{\mathrm{Pe}}}{s\left[\frac{(1-s)}{\sqrt{2 \mathrm{Pe}}} H_{-s}(\sqrt{2 \mathrm{Pe}})-\left(\frac{1}{4 \mathrm{Pe}}+1\right) H_{1-s}(\sqrt{2 \mathrm{Pe}})\right]}
$$

Substituting in (A-10)

$$
F(r, s)=\frac{\frac{2}{r} H_{1-s}(r \sqrt{\mathrm{Pe}})}{s\left[\frac{(1-s)}{\sqrt{2 \mathrm{Pe}}} H_{-s}(\sqrt{2 \mathrm{Pe}})-\left(\frac{1}{4 \mathrm{Pe}}+1\right) H_{1-s}(\sqrt{2 \mathrm{Pe}})\right]}
$$

As $\tau \rightarrow \infty, s \rightarrow 0$ and

$$
\begin{gathered}
F(r, s) \rightarrow \frac{2 \sqrt{2 \mathrm{Pe}}}{s\left[\frac{1}{\sqrt{2 \mathrm{Pe}}}-\left(\frac{1}{4 \mathrm{Pe}}+1\right) 2 \sqrt{2 \mathrm{Pe}}\right]}=-\frac{1}{s} \\
\Rightarrow f(r, \infty) \rightarrow-1 \\
\Rightarrow g(r, \infty) \rightarrow 0
\end{gathered}
$$

At $r=2$,

$$
F(2, s)=\frac{1}{s\left[\frac{(1-s)}{\sqrt{2 \mathrm{Pe}}} \frac{H_{-s}(\sqrt{2 \mathrm{Pe}})}{H_{1-s}(\sqrt{2 \mathrm{Pe}})}-\frac{1}{4 \mathrm{Pe}}-1\right]}
$$

As $z \rightarrow \infty, H_{v}(z)=(2 z)^{v}\left[1+\mathrm{O}\left(\frac{1}{|z|^{2}}\right)\right][$ Lebedev 1972]. Hence, for Pe $\gg 1$

$$
\begin{gathered}
F(2, s)=\frac{1}{s\left[\frac{(1-s)}{\sqrt{2 \mathrm{Pe}}} \frac{(2 \sqrt{2 \mathrm{Pe}})^{-s}}{(2 \sqrt{2 \mathrm{Pe}})^{1-s}}-\frac{1}{4 \mathrm{Pe}}-1\right]}=\frac{-4 \mathrm{Pe}}{s(s+4 \mathrm{Pe})} \\
\Rightarrow f(2, \tau)=-\left(1-e^{-4 \mathrm{Pe} \tau}\right) \\
\quad \text { or } f(2, t)=-\left(1-e^{-4 \mathrm{Pe}^{2} t}\right)
\end{gathered}
$$




$$
g(2, t)=n(t)\left[1-\left(1-e^{-4 \mathrm{Pe}^{2} t}\right)\right]=n(t) e^{-4 \mathrm{Pe}^{2} t}
$$

\section{Solution for $\mathrm{Pe}<0$}

The pair distribution equation (A-5a) can be made independent of the Peclet number by scaling the distance and time in the following way:

$$
\text { Let } z=r \sqrt{-\mathrm{Pe}} \text { and } \tau=t(-\mathrm{Pe})
$$

Using the above scaling, we get

$$
\begin{gathered}
\frac{\partial^{2} f}{\partial z^{2}}+\frac{2}{z} \frac{\partial f}{\partial z}+z \frac{\partial f}{\partial z}-\frac{\partial f}{\partial \tau}=0 \\
\frac{1}{z}\left(z \frac{\partial^{2} f}{\partial z^{2}}+2 \frac{\partial f}{\partial z}\right)+\left(z \frac{\partial f}{\partial z}+f\right)-f-\frac{\partial f}{\partial \tau}=0 \\
\frac{\partial^{2}(z f)}{\partial z^{2}}+z \frac{\partial(z f)}{\partial z}-z f-z \frac{\partial f}{\partial \tau}=0 \\
\frac{\partial^{2} y}{\partial z^{2}}+z \frac{\partial y}{\partial z}-y-\frac{\partial y}{\partial \tau}=0
\end{gathered}
$$

The boundary conditions become

$$
\begin{aligned}
y & \sim 0 \text { as } z \rightarrow \infty \\
\frac{1}{z} \frac{\partial y}{\partial z}-\frac{f}{z}+2 \sqrt{-\mathrm{Pe}} f & =-2 \sqrt{-\mathrm{Pe}} @ z=2 \sqrt{-\mathrm{Pe}} \\
\Rightarrow \frac{\partial y}{\partial z}-\left(\frac{1}{2 \sqrt{-\mathrm{Pe}}}-2 \sqrt{-\mathrm{Pe}}\right) y & =4 \mathrm{Pe} \quad @ z=2 \sqrt{-\mathrm{Pe}}
\end{aligned}
$$

The above equation can be solved by carrying out a Laplace transform in the time domain.

$$
\begin{gathered}
y(z, \tau) \rightarrow Y(z, s)=r \sqrt{-\operatorname{Pe}} F(r, s) \\
\frac{\partial y}{\partial \tau}=s Y(z, s)-y(z, 0)
\end{gathered}
$$


Initial condition : $y(z, 0)=z f(z, 0)=0$

A Laplace transform of (A-17a) gives

$$
\begin{aligned}
& \frac{\partial^{2} Y}{\partial z^{2}}+z \frac{\partial Y}{\partial z}-(1+s) Y=0 \\
& \text { Let } x=\frac{z}{\sqrt{2}}=r \sqrt{\frac{-\mathrm{Pe}}{2}} \\
& \frac{\partial^{2} Y}{\partial x^{2}}+2 x \frac{\partial Y}{\partial x}-\sigma Y=0 \\
& \text { where } \sigma=2(1+s)
\end{aligned}
$$

The above differential equation can be transformed into Whittaker's equation via the transformation $Y=x^{-1 / 2} e^{-x^{2} / 2} W(\xi)$ where $\xi=x^{2}, \kappa=-\left[\frac{\sigma}{4}+\frac{1}{4}\right]=-\frac{s}{2}-\frac{3}{4}$ and $\mu=\frac{1}{4}$. The modified equation is written as

$$
\frac{\partial^{2} W}{\partial \xi^{2}}+\left[-\frac{1}{4}+\frac{\kappa}{\xi}+\frac{\left(\frac{1}{4}-\mu^{2}\right)}{\xi^{2}}\right] W=0
$$

The general solution of this equation satisfying the boundary condition as $z \rightarrow \infty$ is given by

$$
\begin{aligned}
W_{\kappa, \mu}(\xi) & =A e^{-\xi / 2} \xi^{\mu+\frac{1}{2}} U\left(\frac{1}{2}+\mu-\kappa, 1+2 \mu, \xi\right) \\
\Rightarrow Y(x, s) & =A x e^{-x^{2}} U\left(\frac{s}{2}+\frac{3}{2}, \frac{3}{2}, x^{2}\right) \\
\Rightarrow F(r, s) & =\frac{A}{\sqrt{2}} e^{\frac{\mathrm{Pe}}{2} r^{2}} U\left(\frac{s}{2}+\frac{3}{2}, \frac{3}{2},-\frac{\mathrm{Pe}}{2} r^{2}\right)
\end{aligned}
$$

where $U(a, b, z)$ is the Confluent Hypergeometric Function of the second kind. A Laplace transform of the boundary condition at $r=2$ results in

$$
\frac{\partial F}{\partial r}-2 \mathrm{Pe} F=\frac{2 \mathrm{Pe}}{s} \quad @ r=2
$$

Using $U^{\prime}(a, b, z)=-a U(a+1, b+1, z)$ [Abramowitz and Stegun 1964], we get

$$
A=\frac{\sqrt{2}}{s e^{2 \mathrm{Pe}}\left(\frac{s}{2}+\frac{3}{2}\right) U\left(\frac{s}{2}+\frac{5}{2}, \frac{5}{2},-2 \mathrm{Pe}\right)}
$$


Substituting in (A-21)

$$
F(r, s)=\frac{e^{\frac{\mathrm{Pe}}{2} r^{2}} U\left(\frac{s}{2}+\frac{3}{2}, \frac{3}{2},-\frac{\mathrm{Pe}}{2} r^{2}\right)}{s e^{2 \mathrm{Pe}}\left(\frac{s}{2}+\frac{3}{2}\right) U\left(\frac{s}{2}+\frac{5}{2}, \frac{5}{2},-2 \mathrm{Pe}\right)}
$$

As $\tau \rightarrow \infty, s \rightarrow 0$ and using $U(a, a, z)=e^{z} \Gamma(1-a, z)$ [Abramowitz and Stegun 1964], we get

$$
\begin{gathered}
F(r, s) \rightarrow \frac{2}{3 s} \frac{\Gamma\left(-\frac{1}{2},-\frac{\mathrm{Pe}}{2} r^{2}\right)}{\Gamma\left(-\frac{3}{2},-2 \mathrm{Pe}\right)} \\
\Rightarrow f(r, \infty) \rightarrow \frac{2}{3} \frac{\Gamma\left(-\frac{1}{2},-\frac{\mathrm{Pe}}{2} r^{2}\right)}{\Gamma\left(-\frac{3}{2},-2 \mathrm{Pe}\right)}=\frac{-\frac{2 \mathrm{Pe}}{r} e^{\frac{\mathrm{Pe}}{2} r^{2}}+\mathrm{Pe} \sqrt{-2 \mathrm{Pe} \pi} \operatorname{erfc}\left(r \sqrt{-\frac{\mathrm{Pe}}{2}}\right)}{\left(\mathrm{Pe}+\frac{1}{4}\right) e^{2 \mathrm{Pe}}-\mathrm{Pe} \sqrt{-2 \mathrm{Pe} \pi} \operatorname{erfc}(\sqrt{-2 \mathrm{Pe}})}
\end{gathered}
$$

In general, at $r=2$,

$$
F(2, s)=\frac{U\left(\frac{s}{2}+\frac{3}{2}, \frac{3}{2},-2 \mathrm{Pe}\right)}{s\left(\frac{s}{2}+\frac{3}{2}\right) U\left(\frac{s}{2}+\frac{5}{2}, \frac{5}{2},-2 \mathrm{Pe}\right)}
$$

As $z \rightarrow \infty, U(a, b, z)=z^{-a}\left[1+\mathrm{O}\left(\frac{1}{|z|}\right)\right]$ [Abramowitz and Stegun 1964]. Hence, for $|\mathrm{Pe}| \gg 1$

$$
\begin{gathered}
F(2, s)=\frac{e^{2 \mathrm{Pe}}(-2 \mathrm{Pe})^{-\frac{s}{2}-\frac{3}{2}}}{s e^{2 \mathrm{Pe}}\left(\frac{s}{2}+\frac{3}{2}\right)(-2 \mathrm{Pe})^{-\frac{s}{2}-\frac{5}{2}}}=\frac{-4 \mathrm{Pe}}{s(s+3)} \\
\Rightarrow f(2, \tau)=-\frac{4}{3} \mathrm{Pe}\left(1-e^{-3 \tau}\right) \\
\text { or } f(2, t)=-\frac{4}{3} \mathrm{Pe}\left(1-e^{3 \mathrm{Pe} t}\right) \\
g(2, t)=n(t)\left[1-\frac{4}{3} \mathrm{Pe}\left(1-e^{3 \mathrm{Pe} t}\right)\right] \sim-n(t)\left[\frac{4}{3} \mathrm{Pe}\left(1-e^{3 \mathrm{Pe} t}\right)+\mathrm{O}(1)\right]
\end{gathered}
$$




\section{Chapter 3}

\section{The Bulk-Viscoelasticity of Suspensions}

\subsection{Introduction}

The monopolar nature of the microstructural disturbance in compression flows of suspensions has important implications for the time-dependent behavior of the suspension rheology. In this chapter we probe the temporal response of bulk suspension stress in the linear response regime of small rates of deformation. This is accomplished by applying a spatially uniform small rate of expansion that is oscillating in time and studying the frequency-dependent bulk viscosity. Most complex fluids exhibit viscoelastic behavior in oscillatory shearing and we expect that viscoelasticity will also be observed in oscillatory compression/expansion. On application of an oscillatory forcing the suspension microstructure cannot always comply instantaneously to the imposed flow, and the outof-phase portion of the microstructural disturbance resists the forcing in an elastic manner. The portion that does deform along with the imposed flow provides the viscous response, hence the combined response is viscoelastic and the relative importance of the viscous response to the elastic response depends on the frequency of oscillation. The viscoelasticity of suspensions in shear flow has been studied extensively, see, e.g., Russel et al. [1989], and recently the theory for the "microviscoelasticity' was also established [Khair and Brady 2005]. Here we determine the viscoelastic response for the third kind of viscosity — the bulk viscosity.

The frequency response of the suspension stress is especially relevant for the bulk viscosity because the predominant method of measuring the bulk viscosity of materials is by applying an oscillating deformation by means of sound waves and measuring the energy dissipation for waves of different frequency. This technique circumvents the problem of changing concentration of the sus- 
pension if a steady compression or expansion flow is applied to probe the rheology. The frequencydependent response can also be thought of as a Fourier transform of the temporal response. Hence the viscoelastic response of the suspension also provides valuable information about the temporal behavior of the microstructure and stress relaxation. We begin with deriving the expressions for the frequency-dependent bulk viscosity in the next section and in later sections we study the frequency response and the temporal response with and without hydrodynamic interactions between particles.

\subsection{Bulk viscosity of a suspension}

We begin by defining the bulk viscosity of a suspension as done in Chapter $2 \S 2.2$, by computing the average stress in the material in a way analogous to that for the shear viscosity [Batchelor and Green 1972b; Brady and Bossis 1988; Brady et al. 2006]. The bulk viscosity is the scalar coefficient that when multiplied with the average rate of expansion gives the difference between the trace of the mean suspension stress and the equilibrium stress. The effective bulk viscosity $\kappa_{e f f}$ is given by

$$
\kappa_{e f f} \equiv \kappa+\left(-\left\langle p_{t h}\right\rangle_{f}+\left\langle p_{t h}\right\rangle_{f}^{e q}\right) /\langle e\rangle+\frac{1}{3} n\left[\left(\left\langle S^{B}\right\rangle-\left\langle S^{B}\right\rangle^{e q}\right)+\left(\left\langle S^{P}\right\rangle-\left\langle S^{P}\right\rangle^{e q}\right)+\left\langle S^{E}\right\rangle\right] /\langle e\rangle,
$$

where $p_{t h}$ is the thermodynamic pressure in the fluid, and $\left\langle S^{B}\right\rangle,\left\langle S^{P}\right\rangle$ and $\left\langle S^{E}\right\rangle$ are the trace of the average particle stresslets due to Brownian motion, interparticle forces and the imposed hydrodynamic flow respectively. The angle brackets $\langle\ldots\rangle$ denote an average over the entire suspension (particles plus fluid), and $\langle\ldots\rangle_{f}$ denotes an average over the fluid phase only. The Brownian and interparticle force contributions arise from interactions between at least two particles and therefore contribute $O\left(\phi^{2}\right)$ to the bulk viscosity. The stresslet due to the imposed rate-of-strain $\left\langle\boldsymbol{S}^{E}\right\rangle$ is nonzero for a single particle and therefore contributes $O(\phi)$ to the bulk viscosity. Calculation of the average particle stresslets requires the knowledge of the particle microstructure so that the stress can be averaged over it.

For dilute suspensions, the particle microstructure can be described using the pair-distribution function $g(r, t)$ which represents the probability of finding a second particle at a distance $r$ relative to another particle in the suspension, at any time $t$. The governing equation for the pair-distribution 
function is the Smoluchowski equation derived in Chapter $2 \S 2.3$ :

$$
\begin{gathered}
\frac{\partial g}{\partial t}+P e_{b} r \frac{\partial g}{\partial r}+P e_{b} \frac{1}{r^{2}} \frac{\partial}{\partial r} r^{2} v^{\prime}(r \hat{b}) g=\frac{1}{r^{2}} \frac{\partial}{\partial r} r^{2} G(r \hat{b}) \frac{\partial g}{\partial r}, \\
G(r \hat{b}) \frac{\partial g}{\partial r}=P e_{b}\left[2+v^{\prime}(2 \hat{b})\right] g \quad \text { at } \quad r=2,
\end{gathered}
$$

and

$$
g \sim 1 \quad \text { as } \quad r \rightarrow \infty,
$$

where $G(r \hat{b})=x_{22}^{a}-x_{21}^{a}$ [Kim and Karrila 1991] is the scalar function for the radial component of the relative mobility of two particles, $\boldsymbol{D}=2 D[G(r \hat{b}) \hat{\boldsymbol{r}} \hat{\boldsymbol{r}}+H(r \hat{b})(\boldsymbol{I}-\hat{\boldsymbol{r}} \hat{\boldsymbol{r}})]$ [Batchelor 1976]. The interparticle disturbance velocity denoted as $v^{\prime}(r \hat{b})$ can also be expressed in terms of the hydrodynamic functions as

$$
v^{\prime}(r \hat{b})=\frac{4}{3} \frac{1}{\hat{b}} G(r \hat{b})\left(X_{22}^{P}-X_{21}^{P}\right)
$$

where $X_{\alpha \beta}^{P}$ are the hydrodynamic resistance functions relating the pressure to the velocity as determined by Jeffrey et al. [1993]. The relative diffusivity is scaled by its value at large separations, $2 D$. The Péclet number is based on the particle radius $b$ and is given by

$$
P e_{b}=\frac{\frac{1}{3}\langle e\rangle b^{2}}{2 D}
$$

where $D=k T / 6 \pi \eta a$ is the Stokes-Einstein-Sutherland diffusivity of an isolated particle. The time is nondimensionalized by the diffusive time $b^{2} / 2 D$. The Péclet number can be positive or negative corresponding to expansion or contraction, respectively.

The above equation for the pair-distribution function is valid for all Péclet numbers. In order to study the viscoelastic response of the suspension to an expansion flow, we consider a rate of expansion that is uniform in space but oscillatory in time $\langle e\rangle=S e^{i \alpha t}$, where $\alpha=\omega b^{2} / 2 D$ is the oscillatory frequency $\omega$ nondimensionalized with the diffusive time-scale $b^{2} / 2 D$. The Péclet number is now based on the amplitude of the oscillatory expansion flow and defined as $P e_{b}=$ $\frac{1}{3} S b^{2} / 2 D$. Additionally we restrict this analysis to the linear response regime for small departures from equilibrium $\left(\left|P e_{b}\right| \ll 1\right)$ such that the amplitude of oscillation is small. In this regime the perturbation to the equilibrium microstructure is linear in the external forcing, so to leading order 
in $P e_{b}$ we may write the pair distribution function as:

$$
g=1+P e_{b} f(r, \alpha) e^{i \alpha t},
$$

where $f$ is the scaled perturbation to the microstructure satisfying to first order in $P e_{b}$ :

$$
\begin{gathered}
i \alpha f+\frac{1}{r^{2}} \frac{\partial}{\partial r}\left(r^{2} v^{\prime}(r \hat{b})\right)=\frac{1}{r^{2}} \frac{\partial}{\partial r}\left(r^{2} G(r \hat{b}) \frac{\partial f}{\partial r}\right), \\
G(r \hat{b}) \frac{\partial f}{\partial r}=2+v^{\prime}(2 \hat{b}) \quad \text { at } \quad r=2,
\end{gathered}
$$

and

$$
f \sim 0 \quad \text { as } \quad r \rightarrow \infty
$$

As the oscillatory expansion flow proceeds the average number density of particles also oscillates in time according to (2.23), and the spatial disturbance in the microstructure relative to the average number density is given by $f(r, \alpha)$.

The deviation of the trace of the bulk stress from its equilibrium value divided by the average rate of expansion gives the bulk viscosity of the suspension, which to second order in volume-fraction of particles can be written as [Brady et al. 2006]:

$$
\kappa_{e f f}=\left(\kappa+\frac{4}{3} \eta \phi\right) \frac{1}{1-\phi}+\kappa^{E}+\kappa^{B}+\kappa^{P}
$$

where the last three terms are the $O\left(\phi_{b}^{2}\right)$ contributions to the bulk viscosity corresponding to the imposed rate-of-strain, Brownian motion and interparticle forces, respectively. It should be noted that the volume fraction for the two-particle contributions is based on the excluded-volume radius $b$ of the particles. The explicit forms for these contributions are given by

$$
\begin{aligned}
\kappa^{B} & =-\eta \frac{\phi_{b}^{2}}{\hat{b}^{2}} \frac{1}{2} \int_{2}^{\infty}\left[\frac{\mathrm{d}}{\mathrm{d} r}\left[X_{22}^{P} x_{22}^{a}+X_{21}^{P} x_{21}^{a}\right]+\frac{2}{r}\left[X_{22}^{P} x_{22}^{a}+X_{21}^{P} x_{21}^{a}\right]\right]_{r \hat{b}} f(r) r^{2} \mathrm{~d} r, \\
\kappa^{P} & =-\eta \frac{\phi_{b}^{2}}{\hat{b}} \frac{3}{2}\left(2+v^{\prime}(2 \hat{b})\right) f(2), \\
\kappa^{E} & =\eta \frac{\phi_{b}^{2}}{\hat{b}^{3}} 2 \int_{2}^{\infty}\left[\left(T_{12}^{Q}+T_{22}^{Q}\right)^{\prime}-\frac{1}{4} \hat{b}\left(X_{22}^{P}-X_{21}^{P}\right) v^{\prime}(r \hat{b})\right]\left(1+P e_{b} f(r)\right) r^{2} \mathrm{~d} r,
\end{aligned}
$$


where $x_{\alpha \beta}^{a}$ are the non-dimensional scalar mobility functions relating velocity to force [Kim and Karrila 1991]. All the hydrodynamic functions are evaluated at $r \hat{b}$ and the prime on $\left(T_{12}^{Q}+T_{22}^{Q}\right)$ is a reminder that the isolated particle value has been removed. All the two-particle contributions are positive and are proportional to the shear viscosity $\eta$ as they arise from the incompressible disturbance flow caused by the presence of the rigid particles.

In the linear-response regime the hydrodynamic contribution $\left(\kappa^{E}\right)$ involves averaging over the equilibrium microstructure $\left(O\left(P e_{b}\right)\right.$ terms are neglected) and so it is a purely real quantity independent of $\alpha$. The interparticle-force and Brownian contributions involve weighting over the perturbed microstructure $f(r, \alpha)$, which is a complex function, and therefore they have a real and an imaginary part. The real part corresponds to the viscous response to the imposed forcing and the imaginary part gives the elastic response. Therefore it is useful to separate the effective bulk viscosity into a real and an imaginary part as:

$$
\kappa_{e f f}(\alpha)=\kappa^{\prime}(\alpha)-i \kappa^{\prime \prime}(\alpha)
$$

The real part of the effective bulk viscosity is

$$
\kappa^{\prime}(\alpha)=\kappa_{0}+\kappa_{E}+\kappa_{B}^{\prime}(\alpha)+\kappa_{P}^{\prime}(\alpha)
$$

where $\kappa_{0}=\left(\kappa+\frac{4}{3} \eta \phi\right) \frac{1}{1-\phi}$ is the $O(\phi)$ bulk viscosity and $\kappa_{B}^{\prime}$ and $\kappa_{P}^{\prime}$ are the real parts of $\kappa_{B}$ and $\kappa_{P}$, respectively. The direct rate-of-strain contribution is purely hydrodynamic and therefore has no frequency dependence as discussed above. The imaginary part of the effective bulk viscosity is

$$
\kappa^{\prime \prime}(\alpha)=\kappa_{B}^{\prime \prime}(\alpha)+\kappa_{P}^{\prime \prime}(\alpha)
$$

where $\kappa_{B}^{\prime \prime}$ and $\kappa_{P}^{\prime \prime}$ are the real parts of $\kappa_{B}$ and $\kappa_{P}$, respectively. The normalized elastic bulk modulus of the suspension $K^{\prime}$ is related to the imaginary part of the bulk viscosity by $K^{\prime}(\omega)=K_{0}+\omega K^{\prime \prime}(\omega)$ [Zwanzig and Mountain 1965], where $K_{0}$ is the normalized zero-frequency bulk modulus (inverse compressibility) of the suspension given by

$$
K_{0}=-V\left(\frac{\partial P}{\partial V}\right)_{s}
$$


The frequency dependent effective bulk viscosity can be computed for all frequencies by solving (3.4) for $f(r, \alpha)$ and substituting in the expressions in (3.6). However, it is instructive to consider the nature of the bulk viscosity in the high and low frequency limits. The high-frequency limit is realized when the frequency of oscillation $\omega$ is much larger than the diffusive time-scale of the particles $b^{2} / 2 D$, corresponding to $\alpha \rightarrow \infty$. The forcing in this case is at such a high frequency that the suspension microstructure is not perturbed from its equilibrium state at all, or $f(r, \infty)=0$. Therefore only the direct hydrodynamic contribution remains and the bulk viscosity is purely real and given by

$$
\kappa_{e f f}(\infty)=\kappa_{0}+\kappa_{E} .
$$

The high-frequency bulk viscosity has no imaginary component and is therefore purely dissipative in nature. On the other hand the low-frequency limit corresponds to a steady rate of expansion (or compression), for which $f$ is purely real as calculated by Brady et al. [2006], and so the bulk viscosity is also purely real. The zero-frequency bulk viscosity is completely dissipative in nature and given by

$$
\kappa_{e f f}(0)=\kappa_{0}+\kappa_{E}+\kappa_{B}^{\prime}(0)+\kappa_{P}^{\prime}(0)
$$

To isolate the frequency dependence of the bulk viscosity it is useful to define reduced bulk viscosity functions. The reduced function for the real part of the bulk viscosity is defined as

$$
\kappa_{r}^{\prime}(\alpha) \equiv \frac{\kappa^{\prime}(\alpha)-\kappa_{e f f}(\infty)}{\kappa_{e f f}(0)-\kappa_{e f f}(\infty)}=\frac{\kappa_{B}^{\prime}(\alpha)+\kappa_{P}^{\prime}(\alpha)}{\kappa_{B}^{\prime}(0)+\kappa_{P}^{\prime}(0)}
$$

and the reduced function for the imaginary part of the bulk viscosity is

$$
\kappa_{r}^{\prime \prime}(\alpha) \equiv \frac{\kappa^{\prime \prime}(\alpha)}{\kappa_{e f f}(0)-\kappa_{e f f}(\infty)}=\frac{\kappa_{B}^{\prime \prime}(\alpha)+\kappa_{P}^{\prime \prime}(\alpha)}{\kappa_{B}^{\prime}(0)+\kappa_{P}^{\prime}(0)}
$$

Both are dimensionless functions of the scaled frequency $\alpha$ only and vary between 0 and 1 . The dependence on volume fraction has also been scaled out. 


\subsection{Microstructure and bulk viscosity: No hydrodynamics}

First we consider the simplest model for the suspension where we neglect hydrodynamic interactions between the particles. In this model each hard sphere behaves as a colloidal particle undergoing Brownian motion and with a self-diffusivity given by the bare diffusivity, but the effect of the disturbance flow due to a particle on the other particles is neglected. Particles may only interact with each other via hard-sphere collisions. This approach allows us to isolate the effect of Brownian motion and inter-particle forces on the microstructure from the effect of hydrodynamic interactions, and is instructive for understanding the nature of the perturbation. In terms of the excluded-annulus model this limit corresponds to $\hat{b} \rightarrow \infty$ for the computation of all the hydrodynamic functions. Note that in this model for hard spheres with no hydrodynamic interactions there is actually no excluded annulus, rather the spheres actually have a radius $b$ and therefore the bare diffusivity is $D=k T / 6 \pi \eta b$. The parameter $\hat{b}$ only serves to control the strength of hydrodynamic interactions between particles.

In the absence of hydrodynamics there is no disturbance fluid velocity between any particle pair, so $v^{\prime}(r \hat{b})=0$, and the relative radial mobility is simply $G(r \hat{b})=1$. The Smoluchowski equation for $f(r, \alpha)$ now becomes

$$
\begin{gathered}
\frac{1}{r^{2}} \frac{\partial}{\partial r}\left(r^{2} \frac{\partial f}{\partial r}\right)-i \alpha f=0, \\
\frac{\partial f}{\partial r}=2 \quad \text { at } \quad r=2,
\end{gathered}
$$

and

$$
f \sim 0 \quad \text { as } \quad r \rightarrow \infty
$$

This is a modified spherical Bessel differential equation of order 0 after the coordinate transformation $z=\sqrt{i \alpha}$, and its solution satisfying the boundary condition as $r \rightarrow \infty$ is the modified spherical Bessel function of the second kind. Applying the boundary conditions and simplifying, it has the exact solution

$$
f=-\frac{8}{r} \frac{1}{\left(1+z_{0}\right)} e^{z_{0}(1-r / 2)}
$$

where $z_{0}=2 \sqrt{i \alpha}$. In the absence of hydrodynamic interactions only the stress due to interparticle forces contributes to the bulk viscosity, which in the case of hard spheres arises only from hard- 
sphere collisions and is given by

$$
\kappa^{P}(\alpha)=-3 f(2, \alpha) \eta \phi_{b}^{2} .
$$

The single particle contribution to the bulk viscosity is still present. The $O\left(\phi_{b}^{2}\right)$ hydrodynamic $\left(\kappa^{H}\right)$ and Brownian $\left(\kappa^{B}\right)$ contributions are identically zero because they originate from hydrodynamic interactions due to the imposed external forcing and Brownian motion, respectively. The $O\left(\phi_{b}^{2}\right)$ steady expansion bulk viscosity corresponding to $\alpha=0$ is given by $\kappa^{P}(0)=12 \eta \phi_{b}^{2}$. At the other extreme is the high-frequency limit ( $\alpha \rightarrow \infty$ ) where $f \rightarrow 0$ and so there is no interparticle contribution to the bulk viscosity.

The frequency dependent solution for the microstructure can be broken into its real and imaginary parts, from which we obtain the reduced bulk viscosity functions as

$$
\kappa_{r}^{\prime}(\alpha)=\frac{\kappa_{P}^{\prime}(\alpha)}{\kappa_{P}^{\prime}(0)}=-\frac{1}{4} \mathfrak{R} f(2, \alpha)=\frac{1+\beta}{1+2 \beta+2 \beta^{2}},
$$

and

$$
\kappa_{r}^{\prime \prime}(\alpha)=\frac{\kappa_{P}^{\prime \prime}(\alpha)}{\kappa_{P}^{\prime}(0)}=\frac{1}{4} \mathfrak{J} f(2, \alpha)=\frac{\beta}{1+2 \beta+2 \beta^{2}},
$$

where $\beta=\sqrt{2 \alpha}$. Plots of the real and imaginary reduced viscosity functions are shown in Figure 3.1 and Figure 3.2 along with the reduced shear viscosity functions derived by Brady [1993b]. In the high frequency limit $(\alpha \rightarrow \infty)$ both bulk and shear reduced viscosities asymptote to zero with the same dependence on $\alpha$; only the coefficient is different. In the steady expansion/shear limit $(\alpha \rightarrow 0)$ the real part of both viscosities asymptote to 1 and the imaginary part becomes zero as discussed earlier, but the dependence on $\alpha$ is slower for the bulk viscosity. It is useful to examine the low and high frequency asymptotic limits to understand the nature of the microstructural perturbation in expansion flow and its effect on the bulk viscosity.

A Taylor series expansion of (3.13) for small $\alpha$ produces the following asymptotic form of the microstructural deformation at contact:

$$
f(2 ; \alpha) \sim-4\left\{1-(2 \alpha)^{1 / 2}+2(2 \alpha)^{3 / 2}-i\left((2 \alpha)^{1 / 2}-4 \alpha+2(2 \alpha)^{3 / 2}\right)\right\}+O\left(\alpha^{2}\right) .
$$

The first departure from steady state is $O\left(\alpha^{1 / 2}\right)$ and it is present in both the real and imaginary 
parts. Equation (3.18) represents a direct balance between Brownian diffusion and the imposed forcing. The deformation is dominated by Brownian motion except at $r \sim O\left(\alpha^{-1 / 2}\right)$ and larger where diffusion is balanced by the external forcing. In the case of expansion flow the forcing is radial resulting in concentration gradients that are in the radial direction only. Therefore diffusion due to Brownian motion also takes place only radially. The forcing in expansion/compression flow is therefore monopolar and decays as $1 / r$, as is evident from the value of $f(r, \alpha)=-8 / r$ from equation (3.13) in the limit $\alpha \rightarrow 0$. In contrast the microstructural perturbation in shear flow is quadrupolar, decaying as $1 / r^{3}$, as there is accumulation of particles in the compression axis and depletion of particles in the expansion axis, resulting in Brownian diffusion in the radial as well as tangential directions. Hence the nature of the disturbance in shear flow is fundamentally different from that in expansion flow. This is evident in the Smoluchowski equation for shear flow of hard spheres with no hydrodynamic interactions, given by [Brady 1993b; Cichocki and Felderhof 1991]:

$$
\begin{gathered}
\frac{1}{r^{2}} \frac{\partial}{\partial r}\left(r^{2} \frac{\partial f}{\partial r}\right)-6 \frac{f}{r^{2}}-i \alpha f=0, \\
\frac{\partial f}{\partial r}=-2 \quad \text { at } \quad r=2,
\end{gathered}
$$

and

$$
f \sim 0 \quad \text { as } \quad r \rightarrow \infty .
$$

The extra term $-6 f / r^{2}$ stems from interparticle interactions perpendicular to the line joining the centers between two particles in the suspension. Equation (3.18) is a modified spherical Bessel differential equation of order 2 after the coordinate transformation $z=\sqrt{i \alpha}$, and the exact solution for $f(r, \alpha)$ is

$$
f=\frac{32}{3} \frac{1}{r^{3}}\left(\frac{1+z+\frac{1}{3} z^{2}}{1+z_{0}+\frac{4}{9} z_{0}^{2}+\frac{1}{9} z_{0}^{3}}\right) e^{z_{0}(1-r / 2)},
$$

where $z_{0}=2 \sqrt{i \alpha}$. Only the hard-sphere stress contributes to the shear viscosity in the absence of hydrodynamics and so the reduced shear viscosity functions depend only on $f(2, \alpha)$, just like for the bulk viscosity. The shear viscosity is given by [Brady 1993b]

$$
\eta^{P}=\frac{9}{5} f(2, \alpha) \phi_{b}^{2} \eta
$$


Expanding $f(2, \alpha)$ in a Taylor series for small values of $\alpha$ gives the asymptotic form

$$
f(2, \alpha) \sim \frac{4}{3}\left\{1-\frac{16}{81}(2 \alpha)^{2}+\frac{4}{27}(2 \alpha)^{5 / 2}-i\left(\frac{2}{9}(2 \alpha)-\frac{4}{27}(2 \alpha)^{5 / 2}\right)\right\}+O\left(\alpha^{3}\right) .
$$

The first departure from equilibrium is linear in $\alpha$ and is purely imaginary and therefore elastic in nature. The next correction is $O\left(\alpha^{2}\right)$ and is purely real. The more dominant response being out-ofphase with the imposed flow is a consequence of the quadrupolar nature of the disturbance in shear flow.

In the infinite frequency limit $f=0$ but this solution does not satisfy the no-flux boundary condition at contact. The perturbation about $\alpha \rightarrow \infty$ is singular and there is a boundary layer of $O\left(\alpha^{1 / 2}\right)$ around the particle in which diffusion balances the imposed forcing. Rescaling $r$ to get a stretched coordinate for the boundary layer $y=(r-2) \sqrt{i \alpha}$, and neglecting terms of $O\left(\alpha^{-1 / 2}\right)$ the Smoluchowski equation becomes simply

$$
d^{2} f / d y^{2}=f
$$

which has the solution

$$
f(r, \alpha)=-\frac{2}{\sqrt{i \alpha}} e^{-(r-2) \sqrt{i \alpha}},
$$

giving

$$
f(2, \alpha)=-(1-i) \sqrt{2 \alpha}
$$

Equation (3.22) is obtained for both expansion flow and shear flow after neglecting the $O\left(\alpha^{-1 / 2}\right)$ terms. On the scale of the boundary layer the surface of the particle appears flat and consequently there is a one-dimensional balance between the oscillatory forcing and Brownian diffusion in both cases. The boundary condition for shear flow is slightly different, giving $f(2, \alpha)=(1-i) \sqrt{2 \alpha}$. The real and imaginary parts of $f(2, \alpha)$ and hence the shear and bulk viscosities vanish like $\alpha^{-1 / 2}$ as $\alpha \rightarrow \infty$. This slow decay with $\alpha$ implies that the elastic bulk modulus of the suspension given by $K^{\prime}(\omega)=K_{0}^{\prime}+\omega \kappa^{\prime \prime}(\omega)$ diverges as $\alpha^{1 / 2}$ at high frequency; clearly an unphysical result. The hardsphere potential results in a delta function repulsion force at contact, which is responsible for the divergence because the collision between particles is instantaneous and therefore the stress during 
an infinitesimal time-step containing the collision is infinite. The same behavior has been observed for the elastic shear modulus $G^{\prime}(\omega)$ and Brady [1993b] showed that the inclusion of hydrodynamic interactions removes the divergence. In $\S 3.5$ we will show that accounting for hydrodynamics has the same effect on the bulk modulus and it reaches a high-frequency plateau as $\alpha \rightarrow \infty$.

\subsection{Temporal response}

We will now show that the monopolar forcing in expansion flow also affects the temporal response of the suspension, and this connection is made via the frequency dependence of the microstructural perturbation. The frequency-dependent behavior of the microstructure and bulk viscosity can be thought of as the temporal Fourier transform of the corresponding time-dependent behavior in a steady flow. A Fourier transform of 3.2 in the linear-response regime with no hydrodynamics gives

$$
\frac{1}{r^{2}} \frac{\partial}{\partial r} r^{2} \frac{\partial \hat{f}}{\partial r}-i \alpha \hat{f}=0
$$

with boundary conditions

$$
\hat{f} \rightarrow 0 \quad \text { as } \quad r \rightarrow \infty
$$

and

$$
\frac{\partial \hat{f}}{\partial r}=2\left[\pi \delta(\alpha)+\frac{1}{i \alpha}\right] \quad \text { at } \quad r=2
$$

where $\hat{f}(r, \alpha)$ is the temporal Fourier transform of $f(r, \tau)$ and $\alpha$ is now the Fourier-domain frequency. The term inside square brackets in 3.25 comes from the Fourier transform of the Heavisidestep function due to the fact that at $\tau=0$ the system was at equilibrium and at $\tau>0$ a steady compression/expansion flow was imposed. The solution for $\hat{f}(r, \alpha)$ is given by

$$
f=-\frac{8}{r} \frac{1}{\left(1+z_{0}\right)}\left[\pi \delta(\alpha)+\frac{1}{i \alpha}\right] e^{z_{0}(1-r / 2)}
$$

where again $z_{0}=2 \sqrt{i \alpha}$. The only difference between 3.13 and 3.26 is the additional term due to the Heaviside-step function in the Fourier transform analysis. Had we considered a continuously oscillating forcing without an equilibrium initial condition the result from the Fourier analysis would be identical to that from the frequency-dependent equations. Starting from an equilibrium initial 
condition allows us to study how the microstructure approaches the nonequilibrium steady state in the linear response regime. The microstructural disturbance at contact as a function of time is obtained from a Fourier-Laplace inversion of 3.26 as

$$
f(2, \tau)=-4\left(1-e^{\tau / 4} \operatorname{Erfc}\left(\sqrt{\frac{\tau}{4}}\right)\right)
$$

which behaves as $\tau^{1 / 2}$ at short times and asymptotes to its long-time value as $1-\tau^{-1 / 2}$. In contrast the microstructure in a shear flow asymptotes to its final value much faster as $1-\tau^{-5 / 2}$ [Brady 1994]. Thus the monopolar nature of the forcing in expansion/compression flow also manifests in the temporal response as a very slow asymptotic approach to the final nonequilibrium steady-state.

Next we will explore the time dependence of microstructural relaxation of the fluctuations due to Brownian motion. A well known way to calculate transport properties is to use Green-Kubo relations to get them from stress relaxation functions [Green 1952]. For the shear viscosity one would use the shear stress autocorrelation function at equilibrium, and for the bulk viscosity the pressure autocorrelation is used. Although the time-average of the particle-phase stress for a given volumefraction is a constant at equilibrium, Brownian motion produces fluctuations that lead to temporary deviations in the stress. The pressure autocorrelation function depicts how quickly fluctuations in the particle pressure are dissipated in time. Based on the work by Nagèle and Bergenholtz [1998] the Green-Kubo relation for the frequency-dependent bulk viscosity of a suspension due to Brownian motion is given by

$$
\kappa^{B}(\alpha)=\frac{V}{k T} \int_{0}^{\infty}\langle\delta \Pi(0) \delta \Pi(t)\rangle e^{-i \omega t} \mathrm{~d} t
$$

where the angle brackets denote an ensemble average over the equilibrium structure, $V$ is the volume of the system over which the averaging is done, $t$ is the time, and $\delta \Pi(t)$ is the instantaneous deviation of the osmotic pressure of the suspension from its equilibrium value. The high-frequency elastic bulk modulus $K_{\infty}^{\prime}=K^{\prime}(\omega \rightarrow \infty)$ can also be obtained from the pressure autocorrelation function as

$$
K_{\infty}^{\prime}-K_{0}^{\prime}=\frac{V}{k T} \lim _{t \rightarrow 0}\langle\delta \Pi(0) \delta \Pi(t)\rangle
$$

Nondimensionalizing the osmotic pressure with $n k T$ where $n$ is the number density of particles, and time with the diffusive time of the particles to give $\tau=t\left(2 D / b^{2}\right)$, equation (3.28) can be written 
using nondimensionalized quantities as:

$$
\frac{\kappa^{B}(\alpha)}{\eta}=\frac{9}{4} \phi_{b} N \int_{0}^{\infty}\langle\delta \overline{\Pi(0)} \delta \overline{\Pi(t)}\rangle e^{-i \alpha \tau} \mathrm{d} \tau
$$

where $\phi_{b}$ is the volume-fraction of particles, $N$ is the number of particles in volume $V, \eta$ is the fluid shear viscosity and $\overline{\Pi(t)}$ denotes the non-dimensional osmotic pressure. The Green-Kubo formula can be regarded as a one-sided Fourier transform of the pressure autocorrelation function $\langle\delta \overline{\Pi(0)} \delta \overline{\Pi(t)}\rangle$, or equivalently a Laplace transform along the imaginary axis with the transform parameter $s=i \alpha$. Therefore one can obtain the pressure autocorrelation function from an inverse Laplace transform of the frequency-dependent bulk viscosity.

We define a scaled pressure autocorrelation function $\overline{C^{P}}=\langle\delta \overline{\Pi(0)} \delta \overline{\Pi(t)}\rangle N / \phi_{b}$, and using (3.30) we find that it is related to the bulk viscosity as:

$$
\overline{C^{P}}=\frac{4}{9} \mathcal{L}^{-1}\left\{\frac{\kappa(s)}{\eta \phi_{b}^{2}}\right\},
$$

where $\mathcal{L}^{-1}$ denotes the inverse Laplace transform operator. The inverse transform must be evaluated along the imaginary axis because $s=i \alpha$ and $\kappa(s)$ is fully convergent along this contour, therefore it is equivalent to the inverse Fourier transform in $\alpha$. For hard spheres with no hydrodynamic interactions the bulk viscosity is given by (3.14) and therefore we get

$$
\overline{C_{N H}^{P}}=\frac{4}{3} \mathcal{L}^{-1}\left\{\frac{4}{1+\sqrt{4 s}}\right\}=\frac{4}{3}\left(\frac{2}{\sqrt{\pi \tau}}-e^{\tau / 4} \operatorname{Erfc}\left(\sqrt{\frac{\tau}{4}}\right)\right)
$$

where Erfc is the complementary error function and the subscript $N H$ stands for 'No Hydrodynamics'.

For small values of $t$ the pressure autocorrelation function scales as $t^{-1 / 2}$ with the asymptote $\overline{C_{N H}^{P}} \sim 4 / 3(2 / \sqrt{\pi \tau}-1)$, which corresponds to the high-frequency limit of the bulk viscosity. The same short-time scaling is observed for the shear-stress autocorrelation function without hydrodynamics [Brady 1993b; Cichocki and Felderhof 1991]. Both the shear viscosity and the bulk viscosity have the same $\alpha^{-1 / 2}$ high-frequency scaling which manifests in the stress relaxation functions as a $t^{-1 / 2}$ short time scaling for the shear stress and the pressure. This would imply that the elastic bulk modulus given by (3.29) would diverge as $\alpha \rightarrow \infty$, which is not to be expected in real systems. 
Again, the inclusion of hydrodynamic interactions resolves this apparent aphysical behavior and results in a relaxation function that plateaus to a constant as $t \rightarrow 0$.

As $t \rightarrow \infty$ the pressure autocorrelation decays as $\overline{C_{N H}^{P}} \sim(16 / 3 \sqrt{\pi}) \tau^{-3 / 2}$, obtained using an asymptotic expansion of the complementary error function [Abramowitz and Stegun 1964]. This is a very slow long-time decay of the pressure relaxation in comparison to the $t^{-7 / 2}$ decay of the shear stress relaxation function, and is a consequence of the slow $\alpha^{1 / 2}$ dependence of the bulk viscosity as $\alpha \rightarrow 0$. In fact this difference can be traced back to the monopolar nature of the forcing in expansion flow due to which Brownian diffusion is strictly radial and so the microstructural disturbance decays slowly as $1 / r$. In a shear flow the forcing is quadrupolar, there is Brownian diffusion in the radial as well as tangential directions around the particle, and so the disturbance in the microstructure is able to dissipate faster as $1 / r^{3}$. The slower spatial decay of the disturbance in expansion flow manifests as a slow $t^{-3 / 2}$ temporal decay of the pressure autocorrelation. Figure 3.3 shows the scaled theoretical stress-autocorrelation curves for the particle-pressure as well as the shear stress in the absence of hydrodynamics. The analytical Laplace inversion of the shear viscosity as given by (3.20) is not straightforward, therefore the relaxation curve for the shear stress was obtained via numerical Laplace inversion of the frequency-dependent shear viscosity. It is apparent that there is a wide gap between the long-time tails of the pressure and shear-stress relaxation function. Additionally, the pressure autocorrelation function takes much longer to reach its asymptotic decay of $t^{-3 / 2}$.

\subsection{Effect of hydrodynamic interactions}

Neglecting hydrodynamic interactions allowed us to solve the Smoluchowski equation (3.4) exactly and obtain an analytical expression for the bulk viscosity, but it led to some aphysical behavior in the high-frequency limit for the elastic bulk modulus and in the short-time limit for the stress autocorrelation function. In the high-frequency limit the interactions between particles that are touching or nearly touching dominate the microstructure and stress. Hydrodynamic interactions serve to dampen the particle collisions because the particles never actually come into contact with each other due to lubrication flows between nearly touching particle surfaces, therefore the hardsphere potential plays no dynamic role. However, the strong lubrication forces arising from the no- 
slip boundary condition at contact ensure that the particles behave as hard spheres [Brady 1993a]. In the presence of hydrodynamics there is also an additional contribution from many-body far-field interactions between all the particles.

It is useful to study the asymptotic behavior of the Smoluchowski equation (3.4) with hydrodynamics $(\hat{b} \rightarrow 1)$ to understand the effect of hydrodynamic interactions on the frequency dependence of the bulk viscosity. In the low-frequency limit $(\alpha \rightarrow 0)$ the perturbation to the microstructure is dominated by Brownian diffusion as in the no-hydrodynamics case. The perturbation in the steady expansion limit $(\alpha=0)$ was derived by Brady et al. [2006] as the quadrature

$$
f(r)=-\int_{r}^{\infty} \frac{1}{G(r \hat{b})}\left[\frac{8}{r^{2}}+v^{\prime}(r \hat{b})\right] \mathrm{d} r .
$$

In the absence of hydrodynamics $\hat{b} \rightarrow \infty, v^{\prime}=0$ and $G(r \hat{b})=1$ and the solution reduces to

$$
f_{N H}(r)=-\frac{8}{r}
$$

where the subscript $N H$ stands for 'No Hydrodynamics'. This is the limiting value of $f(r, \alpha)$ in (3.13) as $\alpha \rightarrow 0$. In the presence of a small nonzero oscillatory frequency $\alpha$ the microstructural disturbance is a slight deviation from the steady state result, and therefore has a monopolar forcing decaying as $1 / r$ both with and without hydrodynamics. At large distance $\left(r \sim \alpha^{-1 / 2}\right)$ where diffusion balances the imposed forcing, the strength of hydrodynamic interactions is negligible and so we expect the solution in this outer region to have the same $\alpha^{1 / 2}$ dependence as without hydrodynamics. The perturbation closer to the particle must also match the outer solution further away so the bulk viscosity must have a $\alpha^{1 / 2}$ dependence at low frequencies. The monopolar forcing is reflected in the $\alpha^{1 / 2}$ dependence of the microstructural disturbance at small frequencies of oscillation in both cases. Consequently we expect the pressure autocorrelation function in the presence of hydrodynamic interactions to have the same $t^{-3 / 2}$ long time behavior as without hydrodynamics.

The high-frequency limit is more interesting because Brownian diffusion is expected to balance the imposed forcing inside a boundary layer close to the particle surface, but the hydrodynamic mobility function inside the diffusive term itself asymptotes to zero near contact. To leading order there is a balance between the oscillatory forcing and advection due to disturbance flows, giving the 
asymptotic solution $f(r, \alpha)=i W(r \hat{b}) / \alpha$ in the 'outer region' $\left(r \gg \alpha^{-1}\right)$. This solution satisfies the far boundary condition because $v^{\prime}(r \hat{b}) \rightarrow 0$ as $r \rightarrow \infty$ but it does not satisfy the no-flux boundary condition at contact. In the 'inner region' close to particle contact there is a boundary layer where Brownian diffusion balances the oscillatory forcing. Note that in the presence of hydrodynamics there are two small parameters that will set the size of the boundary layer near contact. There is the length scale set by the oscillatory frequency $\alpha$, and there is also the length scale of lubrication interactions $\delta \equiv \hat{b}-1$ and we will explore how the two length scales affect the size of the boundary layer.

Retaining the hydrodynamic functions, equation (3.4) can be written as

$$
G(r \hat{b}) \frac{\partial^{2} f}{\partial r^{2}}+\left(\frac{2}{r} G(r \hat{b})+\frac{\partial G(r \hat{b})}{\partial r}\right) \frac{\partial f}{\partial r}-i \alpha f=W(r \hat{b})
$$

where the function

$$
W(r \hat{b}) \equiv \frac{1}{r^{2}} \frac{\partial}{\partial r}\left(r^{2} v^{\prime}(r \hat{b})\right)=\frac{\partial v^{\prime}(r \hat{b})}{\partial r}+\frac{2}{r} v^{\prime}(r \hat{b})
$$

is the contribution from disturbance flows around the particle. In the region close to particle contact lubrication forces between particles are significant in the hydrodynamic limit. Therefore the lubrication theory expressions for the hydrodynamic functions must be used in this region, given by [Kim and Karrila 1991; Jeffrey et al. 1993]:

$$
\begin{gathered}
G(r \hat{b}) \simeq 2 \xi+1.8 \xi^{2} \ln \xi+O\left(\xi^{2}\right), \\
\frac{\partial G(r \hat{b})}{\partial r} \simeq \hat{b}(2+3.6 \xi \ln \xi+O(\xi)),
\end{gathered}
$$

and from $(5.28)$

$$
\begin{gathered}
v^{\prime}(r \hat{b}) \simeq-\frac{1}{\hat{b}}\left(2+3 \xi \ln \xi-c_{o} \xi+O\left(\xi^{2}\right)\right), \\
W(r \hat{b})=-\left[3-c_{0}+3 \ln \xi+\frac{2}{r \hat{b}}(2+3 \xi \ln \xi)+O(\xi)\right],
\end{gathered}
$$

where $\xi=r \hat{b}-2$ is the hydrodynamic separation between nearly touching particle surfaces and $c_{0}=4.0653$ is a constant. The no-flux boundary condition at $r=2(2.21)$ using the lubrication form 
of the hydrodynamic functions is

$$
\frac{\partial f}{\partial r}=\frac{1}{2 \hat{b}}\left(1+c_{0}-3 \ln \{2 \delta\}\right)+O(\delta),
$$

where $\delta \ll 1$ when hydrodynamic interactions are important. The mobility coefficient on the lefthand side and the relative velocity on the right-hand side of the boundary condition both asymptote to zero linearly in $\xi$ as $\delta \rightarrow 0$, but the linear dependence cancels out and to leading order the boundary condition specifies the slope of $f(r, \alpha)$ diverging slowly as $\ln \delta$. In the full hydrodynamics limit where $\delta \equiv 0$ both the sides are identically zero, hence the boundary condition is not defined. There is no balance between diffusion due to hard sphere collisions and the imposed forcing at contact because the mobility of touching particles is identically zero. The microstructure is set solely by the interactions due to disturbance flows between particles in this limit. The existence of a thermodynamic boundary layer near contact can therefore be attributed to hard-sphere interactions between particles.

Keeping only the highest order terms in the coefficients, the Smoluchowski equation near particle contact is given by

$$
(2(r-2)+4 \delta) \frac{\partial^{2} f}{\partial r^{2}}+2 \frac{\partial f}{\partial r}-i \alpha f=W(r, \delta)
$$

The separation between particle surfaces is be written as $\xi \simeq r-2+2 \delta$ to leading order in $\delta$ using $\hat{b} \simeq 1$. First let us consider the limit of high frequency and relatively mild hydrodynamic interactions such that $\alpha^{-1} \ll \delta \ll 1$. The innermost boundary layer in this case is of size $\alpha^{-1 / 2}$ and within this region $r-2 \ll \delta$. We define the stretched coordinate $z=(r-2) \sqrt{i \alpha / 4 \delta}$ such that the highest derivative in (3.41) is $O(1)$ in the boundary layer. In order to satisfy the boundary condition (3.40) $f(z, \alpha)$ must scale as $\alpha^{-1 / 2}$ to leading order. Therefore we expand it in inverse powers of $\alpha$ as $f(z, \alpha)=f_{0} \alpha^{-1 / 2}+f_{1} \alpha^{-1}+O\left(\alpha^{-3 / 2}\right)$, substitute it in equation (3.41) and compare like powers of alpha. The resulting equation and boundary conditions for $f_{0}$ are

$$
\begin{gathered}
\frac{\partial^{2} f_{0}}{\partial z^{2}}-f_{0}=0, \\
\frac{\partial f_{0}}{\partial z}=\sqrt{\frac{4 \delta}{i}} A_{0}(\delta) \quad \text { at } \quad z=0,
\end{gathered}
$$




$$
f_{0} \rightarrow 0 \quad \text { as } \quad z \rightarrow \infty
$$

where $A_{0}(\delta)=\left(1+c_{0}-3 \ln \{2 \delta\}\right) / 2$, and have the solution

$$
f_{0}=-\sqrt{\frac{4 \delta}{i}} A_{0}(\delta) e^{-z}
$$

The perturbation decays to zero exponentially on the length scale of the boundary layer and to leading order it scales as $\alpha^{-1 / 2}$. It is also proportional to $\delta^{1 / 2}$ implying that as hydrodynamic interactions become stronger $f_{0} \rightarrow 0$, and to leading order the perturbation will scale as $\alpha^{-1}$ with the solution given by $f_{1}$. A boundary layer solution can also be found for $f_{1}$, and it will asymptote to the outer solution $\left(f_{1} \rightarrow i W(r \hat{b})\right)$ outside the boundary layer. The far-field condition on $f_{1}$ comes from asymptotic matching of the inner and outer solutions. However, it is important to note that when $\delta<\alpha^{-1}$ there will be an inner boundary layer of size $\delta$ and therefore the above analysis is not directly applicable.

Next we investigate the high-frequency limit with strong hydrodynamic interactions $\left(\delta \ll \alpha^{-1} \ll\right.$ 1). First consider the region where $r-2 \ll \delta$. In this region the separation between particle surfaces can be approximated as $\xi \sim 2 \delta$, hence the Smoluchowski equation (3.41) suggests a boundary layer of size $\delta$ in which the derivatives are all $O(1)$. We define a scaled coordinate $y=(r-2) \delta^{-1} / 2$ and expand $f(y, \alpha)$ in powers of $\delta$ as $f=h_{0}+h_{1} \delta+O\left(\delta^{2}\right)$. The resulting equation and boundary conditions are

$$
\begin{gathered}
\frac{\partial^{2}}{\partial y^{2}}\left(h_{0}+h_{1} \delta\right)+\frac{\partial}{\partial y}\left(h_{0}+h_{1} \delta\right)-i \alpha \delta\left(h_{0}+h_{1} \delta\right)=W(y, \delta) \delta, \\
\frac{\partial}{\partial y}\left(h_{0}+h_{1} \delta\right)=2 \delta A_{0}(\delta) \quad \text { at } \quad y=0 .
\end{gathered}
$$

As $y \rightarrow \infty, f$ must asymptote to the solution in the outer boundary layer, which we shall study later. It is evident from matching powers of $\delta$ in equation (3.44) and (3.45) that $h_{0}$ is a constant. The next correction to $f$ will be $O(\delta)$ therefore $h_{0}$ must be the asymptotic value of the outer boundary layer close to contact, which is expected to be $O\left(\alpha^{-1}\right)$, thereby implying that the solution in the next boundary layer must asymptote to a constant near contact. The equation for $h_{1}$ is

$$
\frac{\partial^{2} h_{1}}{\partial y^{2}}+\frac{\partial h_{1}}{\partial y}-i \alpha h_{0}=W(y, \delta)
$$


with boundary conditions

$$
\begin{gathered}
\frac{\partial h_{1}}{\partial y}=2 A_{0}(\delta) \quad \text { at } \quad y=0, \\
h_{1} \rightarrow 0 \quad \text { as } \quad y \rightarrow \infty .
\end{gathered}
$$

For the sake of simplicity we treat $W(y, \alpha)$ as a constant in (3.46) and get the solution

$$
h_{1}=\left(e^{-y}+y\right)\left(W(y, \alpha)+i \alpha h_{0}\right)-2 A_{0} e^{-y} .
$$

Accordingly, the value of $f$ in this region is a constant to leading order, with an $O(\delta)$ correction which serves to satisfy the boundary condition at contact. Thus there is a balance between diffusion due to hard sphere collisions and the imposed forcing in a boundary layer of size $\delta$ near contact which results in an $O(\delta)$ correction to the perturbed microstructure. In the full hydrodynamics limit $(\delta \rightarrow 0)$ this boundary layer disappears completely and particle collisions do not play any role in determining the microstructure.

Finally, in order to find the contact value of $f$ and its behavior outside the thermodynamic boundary layer we need to consider the region where $\delta \ll r-2 \ll \alpha^{-1}$. Here the separation between particle surfaces can be approximated as $\xi \sim r-2$ and the Smoluchowski equation (3.41) now suggests a boundary layer of $\alpha^{-1}$ in which all derivatives are $O(1)$. We define a scaled coordinate $x=(r-2) \alpha$ and expand $f(x, \alpha)$ in inverse powers of $\alpha$ as $f=k_{1} \alpha^{-1}+k_{2} \alpha^{-2}+O\left(\alpha^{-3}\right)$. With this rescaling and comparing like powers of $\alpha^{-1}$ the equation for $k_{1}$ is given by

$$
2 x \frac{\partial^{2} k_{1}}{\partial x^{2}}+2 \frac{\partial k_{1}}{\partial x}-i k_{1}=W(x, \alpha)
$$

with boundary conditions

$$
\begin{gathered}
k_{1} \rightarrow \alpha h_{0} \quad \text { as } \quad x \rightarrow 0, \\
k_{1} \rightarrow i W(x, \alpha) \quad \text { as } \quad x \rightarrow \infty .
\end{gathered}
$$

This is a non-homogeneous modified Bessel differential equation. Again, for simplicity we approximate $W(x, \alpha)$ as a constant in (3.48) and obtain the solution

$$
k_{1}=A_{1} K_{0}(\sqrt{2 i x})+i W(x, \alpha)
$$


where $K_{0}(z)$ is the zeroth order modified Bessel function of the second kind which decays to zero as $x \rightarrow \infty$, and $A_{1}$ is a constant. In the $x \rightarrow 0$ limit we can use the approximation $K_{0}(z) \sim-\ln z$ [Abramowitz and Stegun 1964] and employ the lubrication expression for $W(x, \alpha)$ (3.39). Choosing $A_{1}$ such that $k_{1}$ is constant in the limit $x \rightarrow 0$, we get

$$
k_{1}=-6 i K_{0}(\sqrt{2 i x})+i W(x, \alpha)
$$

with the contact value

$$
k_{1}(0)=-\frac{3 \pi}{2}+i\left(3 \ln (2 \alpha)+c_{0}-5\right)
$$

The imaginary part of the contact value scales as $\alpha^{-1} \ln (\alpha)$ with increasing $\alpha$ while the real part is exactly $-3 \pi / 2 \alpha^{-1}$. However, spatially the real part decays to zero on a length scale $\alpha^{-1}$ while the imaginary part asymptotes to $i W(r \hat{b})$, which has a much slower decay to zero. Therefore the imaginary (or elastic) response dominates in the overall microstructural perturbation at high frequencies.

Thus in the full hydrodynamics limit $(\delta \rightarrow 0)$ the $O\left(\alpha^{-1 / 2}\right)$ disturbance given by $f_{0}$ becomes zero and the leading order disturbance to the microstructure is $O\left(\alpha^{-1}\right)$, given by

$$
f(r, \alpha)=\alpha^{-1}\left(-6 i K_{0}(\sqrt{2 i \alpha(r-2)})+i W(r \hat{b})\right),
$$

and consequently the bulk elastic modulus asymptotes to a constant as $\alpha \rightarrow \infty$ in the limit of full hydrodynamics. With the knowledge of $f(r, \alpha)$ the Brownian and interparticle-force contributions to the bulk viscosity can be evaluated from (3.6). The interparticle-force contribution is zero in the full hydrodynamics limit because the hard sphere collisions constituting the interparticle forces are absent. The Brownian contribution has the same $\alpha^{-1}$ scaling as $f(r, \alpha)$, and accordingly the high frequency elastic bulk modulus given by

$$
K^{\prime}(\alpha)\left(\frac{a^{3}}{k T}\right)=\frac{1}{3 \pi} \alpha \frac{\kappa^{\prime \prime}(\alpha)}{\eta}
$$

plateaus to a constant value as $\alpha \rightarrow \infty$. The high frequency limit of the $K^{\prime}(\alpha)$ was calculated numerically as described in the next section. The inclusion of hydrodynamic interactions corrects the aphysical behavior of the bulk modulus at high frequencies. As a corollary, the short time 
temporal response of the microstructural disturbance is finite in the presence of hydrodynamics, causing the pressure autocorrelation function to asymptote linearly to a constant as $t \rightarrow 0$.

\subsection{Numerical results}

The Smoluchowski equation with full hydrodynamic interactions (3.34) was solved numerically using finite-differences for a wide range of values of $\hat{b} \geq 1$ and $\alpha$ to study the effect of varying strength of hydrodynamic interactions on the frequency-dependent bulk viscosity. The far boundary was chosen at a large value of $r$ such that the solution is expected to be close to round-off error in that region and $f$ was set to zero at the far end. The microstructural perturbation decays slowly as $1 / r$ in the low frequency limit ( $\alpha \rightarrow 0$ ), and decays exponentially near contact on a length scale $\delta$ in the high frequency limit $(\alpha \rightarrow \infty)$. A logarithm transformation of the coordinate was employed to shrink the domain far from particle contact and expand it close to contact, so that there are no extreme gradients in the solution for any value of $\alpha$ or $\delta$. In addition, the finite difference grid was discretized with a geometrically increasing number of points closer to contact where the slope of $f$ is expected to be steep in the $\alpha \rightarrow \infty$ limit, leading to a more accurate solution for $f$. The number of grid points were also calibrated such that all the gradients were captured accurately, and increasing the grid size did not change the results. The transformed coordinate is given by

$$
z=\ln (r-2+\epsilon)
$$

where $\epsilon$ is a small parameter that determines the maximum stretching of the domain occurring at particle contact. As $\epsilon \rightarrow 0$, the region close to contact $(r-2 \rightarrow 0)$ gets stretched more and more because $z \rightarrow-\infty$ at contact. The minimum value of $z$ (at $r=2$ ) is given by $\ln \epsilon$ and controls the coordinate stretching near contact. In the nearly-full-hydrodynamics limit at both high frequencies $\left(\delta \ll \alpha^{-1} \ll 1\right)$ and low frequencies $(\delta \ll \alpha \ll 1)$, a value of $\epsilon \ll \delta$ was chosen so that all the physics in the hydrodynamic boundary layer is captured correctly. For frequencies of the order $\alpha^{-1 / 2} \sim \delta$, values of $\epsilon \sim \delta \alpha^{-1 / 2}$ and lesser were found to give good results. In the absence of hydrodynamics $(\hat{b} \gg 1), \epsilon \ll \alpha^{-1 / 2}$ was used in the high frequency limit while at low frequencies $\epsilon \sim 10^{-2}$ was sufficient to resolve the microsructure near contact correctly. The hydrodynamic 
functions for $r \hat{b}>2.001$ were computed using twin multipole expansion [Jeffrey and Onishi 1984; Jeffrey et al. 1993], and for $r \hat{b}<2.001$ the lubrication theory expressions for the functions were used. The bulk viscosity was computed numerically using (3.6) and the numerical solution for $f(r, \alpha)$.

The zero frequency (steady expansion) bulk viscosity with full hydrodynamic interactions was obtained numerically as $\kappa^{B}(0)=5.247 \phi_{b}^{2} \eta$. Figure 3.4 shows the real reduced bulk viscosity function $\kappa_{r}^{\prime}$ as a function of $\alpha$ with varying levels of hydrodynamic interactions prescribed by $\hat{b}-1=10^{-6}, 10^{-3}, 10^{-2}, 10^{-1}$ and $10^{5}$. As $\alpha \rightarrow 0$ the bulk viscosity asymptotes to its steady expansion value as $\alpha^{1 / 2}$ for all values of $b / a$. It undergoes a 'frequency thinning' as $\alpha$ increases and eventually decays to zero as $\alpha \rightarrow \infty$. In the high frequency limit the microstructure is not perturbed at all and therefore there is no Brownian contribution to the bulk viscosity. In the absence of hydrodynamics $\left(\hat{b}-1=10^{5}\right)$ the real part of the bulk viscosity decays like $\alpha^{-1 / 2}$ as $\alpha \rightarrow \infty$, but with full hydrodynamics the decay is almost $O\left(\alpha^{-2}\right)$. Recall from the previous section that as $\hat{b} \rightarrow 1$ the real part of the microstructural perturbation not only scales as $\alpha^{-1}$ but also decays to zero within a boundary layer of size $\alpha^{-1}$, thereby giving a $\alpha^{-2}$ scaling for the real bulk viscosity obtained by a spatial integration of the Brownian stress weighted with the perturbation. For intermediate values of $\hat{b}$ the transition from the full hydrodynamics scaling to the no-hydrodynamics scaling can be seen as $\alpha$ increases and the scaling is governed by a boundary layer of size $\alpha^{-1 / 2}$.

Figure 3.5 shows a plot of the imaginary reduced bulk viscosity function $\kappa_{r}^{\prime \prime}$ versus $\alpha$ for varying levels of hydrodynamic interactions given by $\hat{b}-1=10^{-6}, 10^{-3}, 10^{-2}, 10^{-1}$ and $10^{5}$. In the low frequency limit the perturbation to the microstructure is mostly in-phase with the imposed forcing, hence the imaginary bulk viscosity vanishes as $\alpha \rightarrow 0$. The frequency dependence does not change with different values of $\hat{b}$ because the microstructure is set by a balance between Brownian diffusion and the oscillatory forcing over the entire domain in all cases. As $\alpha$ increases $\kappa_{r}^{\prime \prime}$ also increases and reaches a peak near $\alpha \sim 0.25$. As $\alpha \rightarrow \infty$ it again decays as $\alpha^{-1 / 2}$ in the absence of hydrodynamic interactions, and as $\alpha^{-1}$ with full hydrodynamics. The imaginary response has the same scaling as the real part when hydrodynamics are not important but as $\hat{b} \rightarrow 1$ the imaginary response dominates with the real part decaying much faster. For intermediate values of $\hat{b}$ one can see a transition from the $\alpha^{-1}$ scaling to the $\alpha^{-1 / 2}$ scaling as $\alpha$ increases.

In the high frequency limit with full hydrodynamic interactions the elastic bulk modulus has 
a finite value calculated to be $K^{\prime}(\infty) \simeq 0.56\left(k T / a^{3}\right) \phi_{b}^{2}$. Figure 3.6 shows the bulk modulus as a function of $\alpha$ for varying levels of hydrodynamic interactions. In the $\hat{b} \rightarrow 1$ limit the bulk modulus asymptotes to a constant as $\alpha \rightarrow \infty$ but for intermediate values of $\hat{b}-1 \ll 1$ the bulk modulus switches scaling from constant to $O\left(\alpha^{1 / 2}\right)$ as $\alpha$ varies from $\alpha^{-1} \gg \hat{b}-1$ to $\alpha^{-1} \ll \hat{b}-1$. In the absence of hydrodynamic interactions $(\hat{b} \gg 1)$ the bulk modulus has a $\alpha^{1 / 2}$ scaling at high frequencies, as discussed previously.

\subsection{Conclusions}

The frequency-dependent bulk viscosity was determined for dilute suspensions for all frequencies both with and without hydrodynamic interactions between particles. Qualitatively it has the same behavior as the shear viscosity and the microviscosity, but the response in the low frequency regime is quite different. This is where the monopolar nature of the pressure relaxation differentiates the bulk viscosity from other rheological properties. Perhaps the most significant find in this work was the determination of an exact analytical expression for the pressure autocorrelation function. Stress autocorrelations are notoriously difficult to study especially at long times, and the pressure autocorrelation function even more so because it decays very slowly. With theoretical knowledge of the pressure autocorrelation function we are now well equipped to analyze the data from numerical simulations and experiments. 


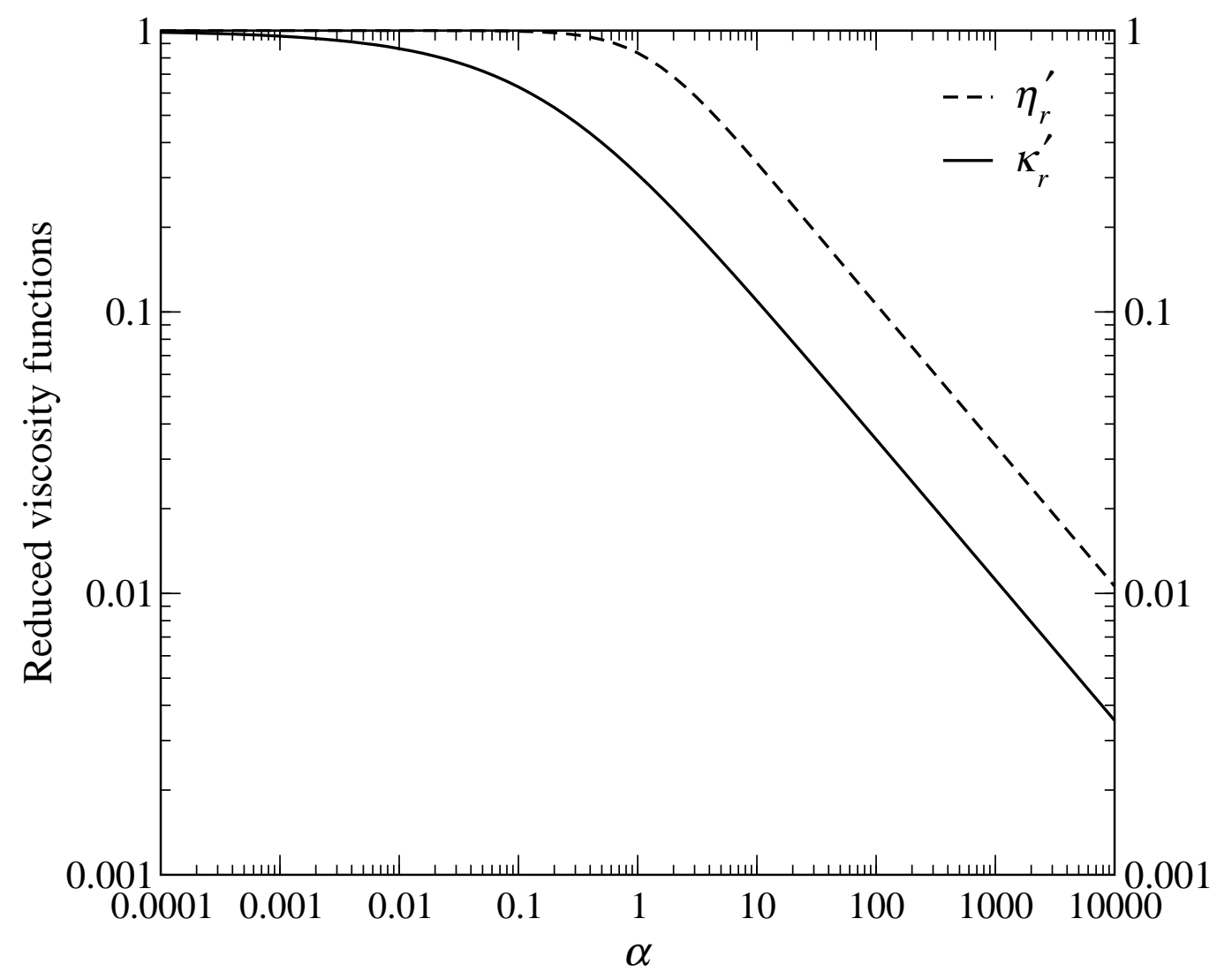

Figure 3.1: Real reduced functions for the bulk viscosity (solid curve) and shear viscosity (dashed curve) of hard spheres with no hydrodynamic interactions. 


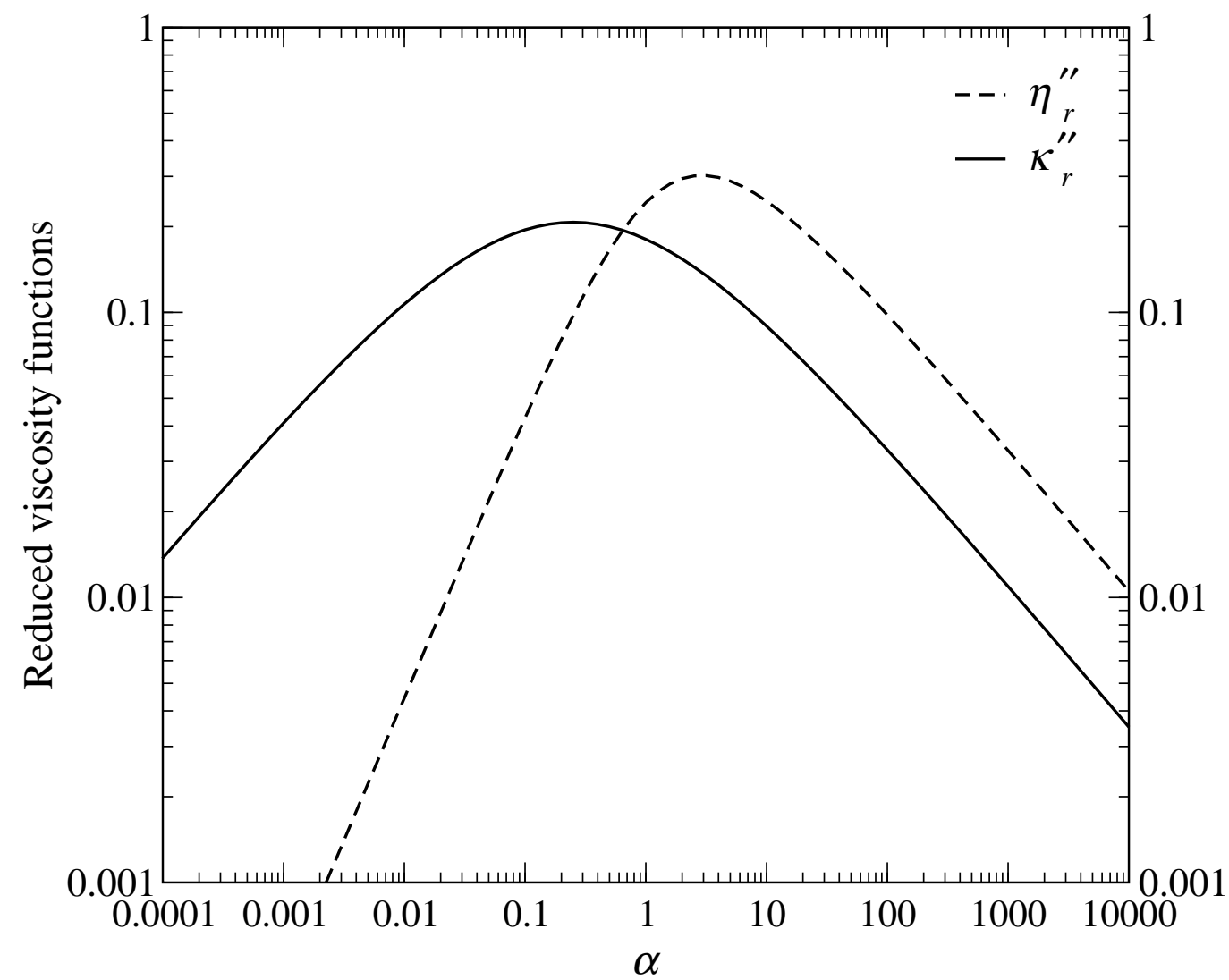

Figure 3.2: Imaginary reduced functions for the bulk viscosity (solid curve) and shear viscosity (dashed curve) of hard spheres with no hydrodynamic interactions. 


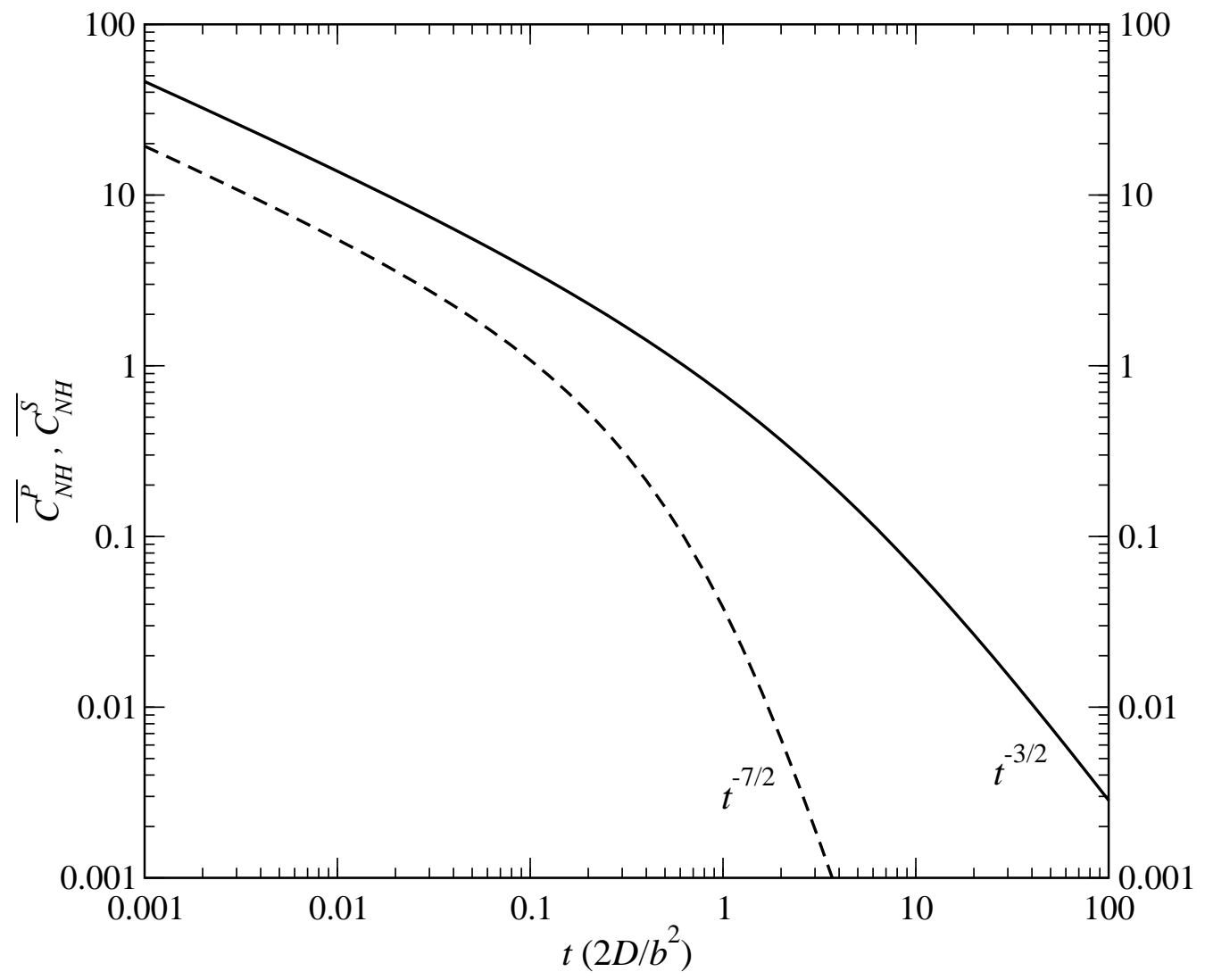

Figure 3.3: The scaled Pressure autocorrelation function $\overline{C_{N H}^{P}}$ (solid curve) for hard spheres with no hydrodynamic interactions. The corresponding shear-stress autocorrelation function $\overline{C_{N H}^{S}}$ (dashed curve) is also shown for comparison. 


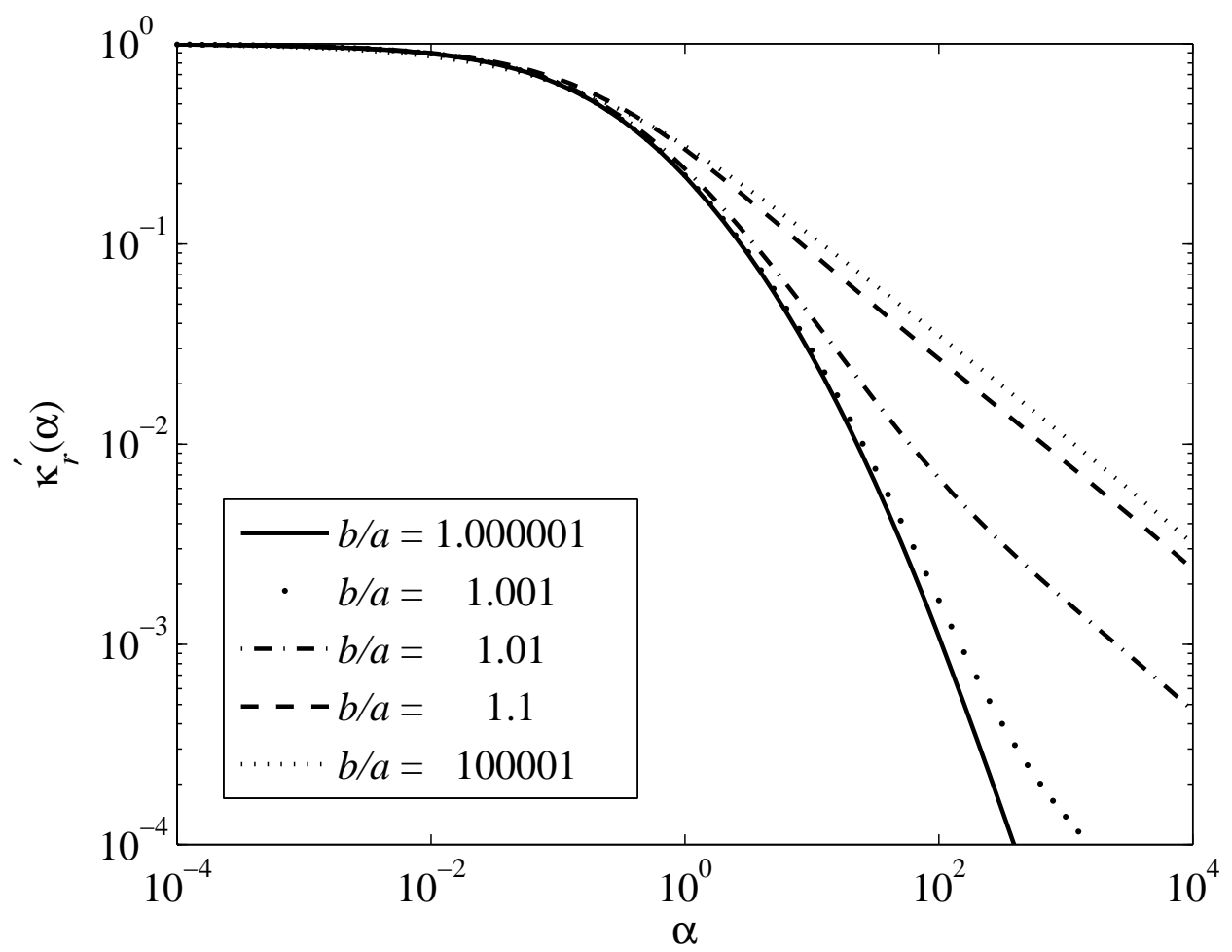

Figure 3.4: Real part of the reduced bulk viscosity function $\kappa_{r}^{\prime}(\alpha)$ as a function of the nondimensional frequency $\alpha=\omega b^{2} / 2 D$ at varying levels of hydrodynamic interactions from nearly full $\left(\hat{b}-1=10^{-6}\right)$ to no hydrodynamics $\left(\hat{b} \simeq 10^{5}\right)$. The steady expansion limit of the bulk viscosity without hydrodynamics is $\kappa^{P}(0)=12 \eta \phi_{b}^{2}$ and with full hydrodynamics $\kappa^{B}(0)=5.247 \eta \phi_{b}^{2}$. 


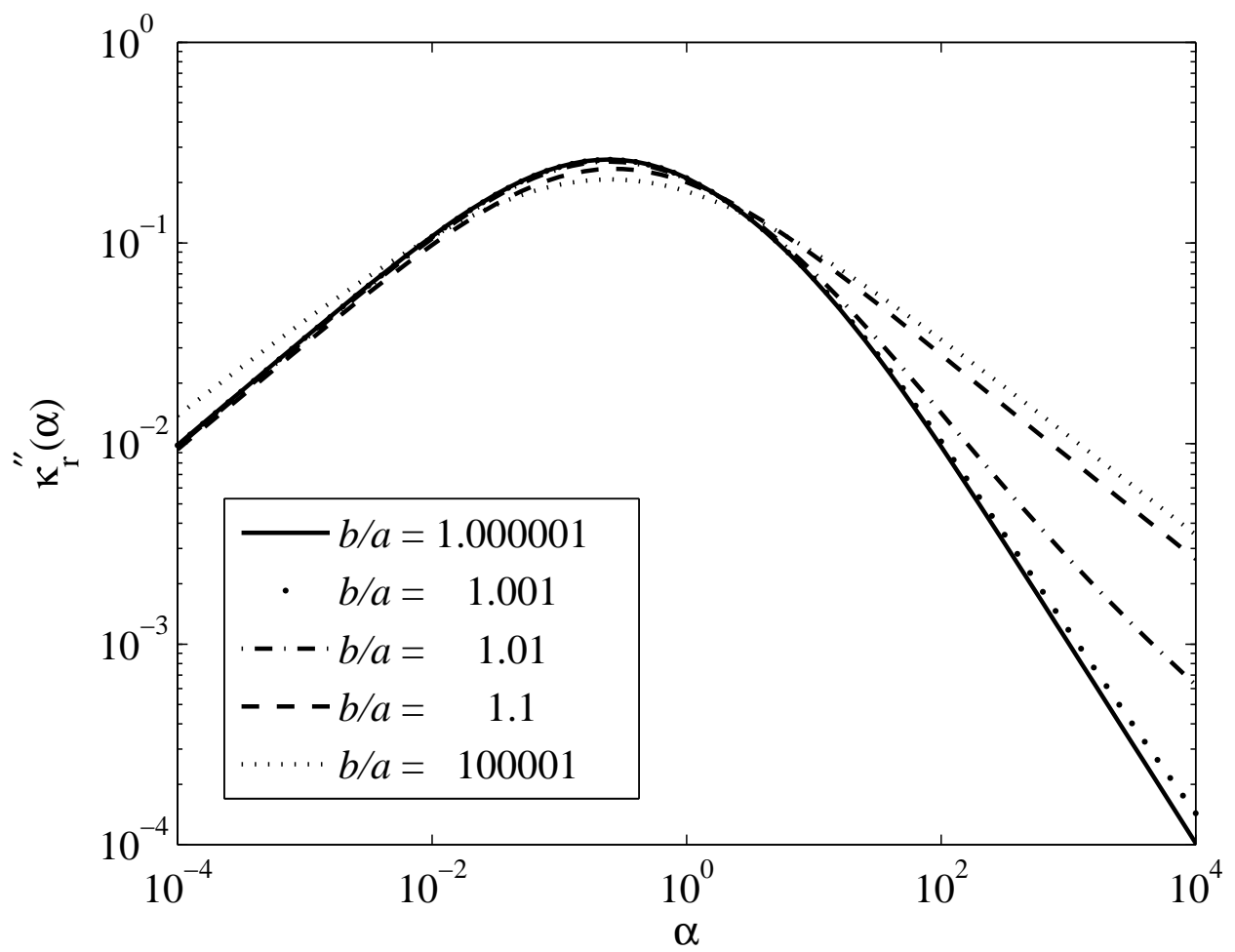

Figure 3.5: Imaginary part of the reduced bulk viscosity function $\kappa_{r}^{\prime \prime}(\alpha)$ as a function of the nondimensional frequency $\alpha=\omega b^{2} / 2 D$ at varying levels of hydrodynamic interactions from nearly full $\left(\hat{b}-1=10^{-6}\right)$ to no hydrodynamics $\left(\hat{b} \simeq 10^{5}\right)$. 


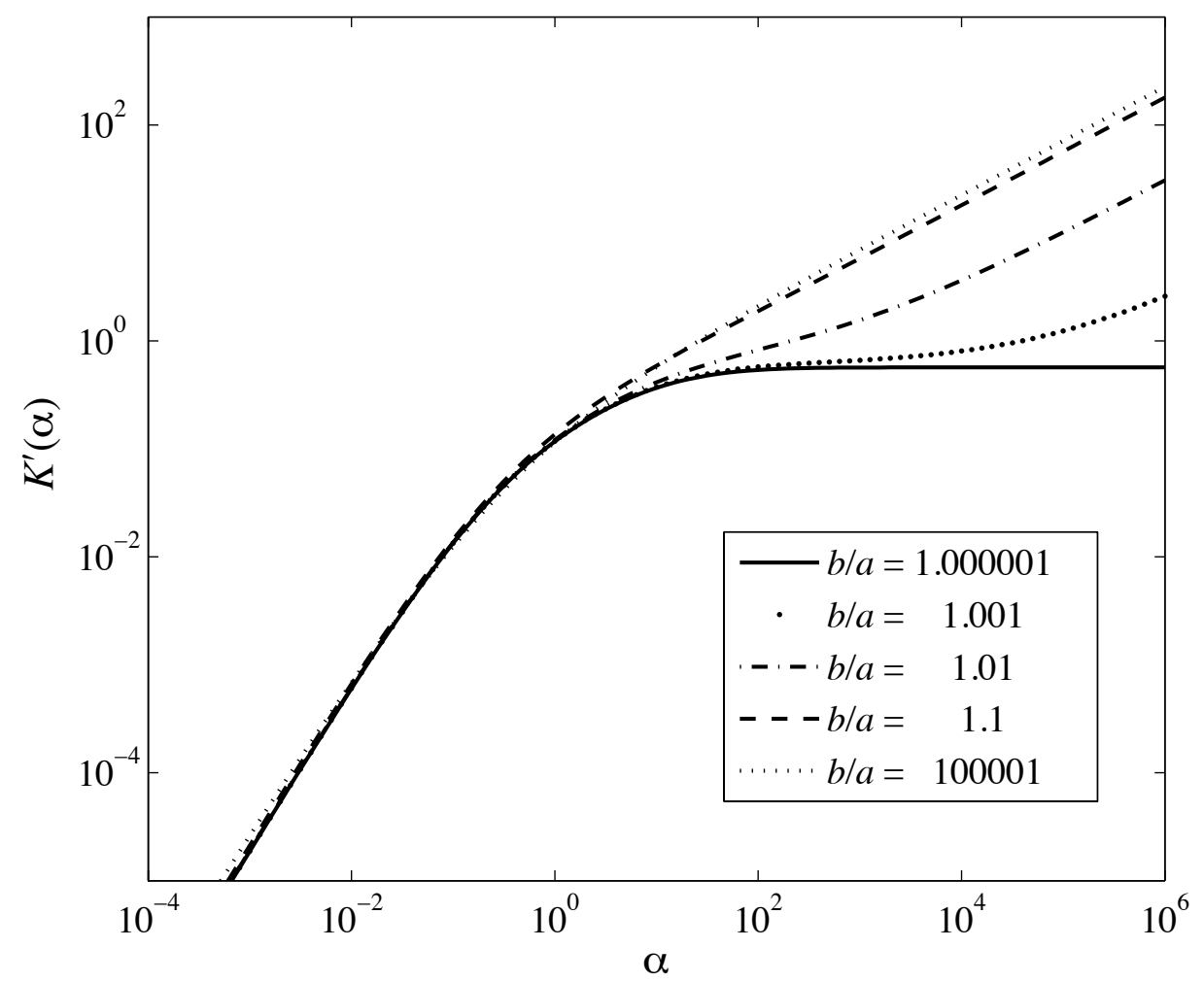

Figure 3.6: The elastic bulk modulus $K^{\prime}(\alpha)$ as a function of the nondimensional frequency $\alpha=$ $\omega b^{2} / 2 D$ at varying levels of hydrodynamic interactions from nearly full $\left(\hat{b}-1=10^{-6}\right)$ to no hydrodynamics $\left(\hat{b} \simeq 10^{5}\right)$. The high-frequency limit with full hydrodynamics is $K^{\prime}(\infty) \simeq 0.56\left(k T / a^{3}\right) \phi_{b}^{2}$. 


\section{Chapter 4}

\section{Stokesian Dynamics Simulations for Compressible Flows of Suspensions}

\subsection{Introduction}

Numerical simulations of particulate suspensions play an important role in the study of complex fluids and multiphase materials. Simulations have been used to make accurate predictions of the macroscopic equilibrium and transport properties of such materials and to study the microstructural mechanics of the particles in a variety of flow situations [Brady and Bossis 1989; Brady and Foss 2000; Sierou and Brady 2002]. So far the simulation work has focused only on incompressible flows of suspensions. More recently Brady, Khair, and Swaroop [2006] determined the 'effective bulk viscosity' (also known as the second or expansion viscosity) for dilute suspensions in a linear expansion flow. The bulk viscosity of a suspension relates the deviation of the trace of the macroscopic stress from its equilibrium value to the average rate of expansion. In this chapter we describe the adaptation of the Stokesian Dynamics (SD) [Durlofsky, Brady, and Bossis 1987] and Accelerated Stokesian Dynamics (ASD) [Sierou and Brady 2001; Banchio and Brady 2003] techniques for simulation of particles in a fluid to allow for expansion flow and calculation of the trace of the particle-phase stress.

Simulating the expansion (or compression) of only the particle phase in a suspension would necessarily lead to spatial variations in the particle volume-fraction, which makes it difficult to determine the bulk viscosity for a given volume-fraction. This problem can be circumvented by having the surrounding fluid expand or compress uniformly in space, leading to a homogeneous expansion or compression of the particle phase as well. The imposed flow causes the suspended 
particles to move apart uniformly in space, but they cannot expand with the fluid, thereby producing a disturbance flow as the fluid has to move around the particles. This disturbance flow causes the stress on the particles to change, also changing the bulk stress in the suspension. The bulk viscosity of the suspension is then determined by computing the deviation in average stress in the material in a way analogous to that for the shear viscosity [Batchelor and Green 1972b; Brady and Bossis 1988], and relating it to the average rate of expansion. We expect the bulk viscosity calculated by this technique to be comparable to that for expansion of the particle phase only, because the contribution to the isotropic stress in both cases is due to incompressible disturbance flows generated due to the finite size of the particles as they move relative to each other. Brady, Khair, and Swaroop [2006] derived the expressions for computing the bulk viscosity for a suspension of rigid particles in a uniformly expanding fluid, and calculated the bulk viscosity for dilute suspensions at small rates of expansion. Subsequently, the bulk viscosity for all rates of compression was determined to second order in volume-fraction by Swaroop and Brady [2007]. At high particle concentrations the manybody interactions between particles play a significant role in determination of the particle motion and lubrication interactions between nearly touching particles comprise the dominant contribution to the bulk stress. This necessitates the use of numerical simulation to calculate the total stress in the suspension. In the following sections we will describe the Stokesian Dynamics and Accelerated Stokesian Dynamics methods for simulation of suspension flows and how they were adapted for linear compressible flows.

\subsection{Suspension stress}

Consider a homogeneous suspension of spherical particles with number density $n$ in a compressible Newtonian fluid of density $\rho$, shear viscosity $\eta$ and bulk viscosity $\kappa$. The particles are small enough that the Reynolds number $R e=\rho U a / \eta$ (with typical velocity $U$ and particle radius $a$ ) is very small, thus enabling the use of Stokes equations. The ensemble or volume average of the Cauchy stress in the suspension is given by [Brady 1993a]

$$
\langle\boldsymbol{\Sigma}\rangle=-\left\langle p_{t h}\right\rangle_{f} \boldsymbol{I}+2 \eta\langle\boldsymbol{e}\rangle+\left(\kappa-\frac{2}{3} \eta\right)\langle\boldsymbol{\nabla} \cdot \boldsymbol{u}\rangle \boldsymbol{I}-n k T \boldsymbol{I}+n\left[\left\langle\boldsymbol{S}^{B}\right\rangle+\left\langle\boldsymbol{S}^{P}\right\rangle+\left\langle\boldsymbol{S}^{E}\right\rangle\right],
$$


where $\boldsymbol{e}$ is the rate of strain in the fluid, $\langle\ldots\rangle$ denotes an average over the entire suspension (particles plus fluid), and $\langle\ldots\rangle_{f}$ denotes an average over the fluid phase only. The trace of $\langle\boldsymbol{e}\rangle$, given by $\langle e\rangle \equiv\langle\boldsymbol{\nabla} \cdot \boldsymbol{u}\rangle$ is the average rate of expansion of the suspension. The average hydrodynamic stresslet $\left\langle\boldsymbol{S}^{H}\right\rangle=(1 / N) \sum_{\alpha=1}^{N} \boldsymbol{S}_{\alpha}^{H}$ is defined as a number average over all particles, where the stresslet of particle $\alpha$ is given by

$$
\boldsymbol{S}_{\alpha}^{H}=\frac{1}{2} \int_{S_{\alpha}}\left[(\boldsymbol{r} \boldsymbol{\sigma} \cdot \boldsymbol{n}+\boldsymbol{\sigma} \cdot \boldsymbol{n r})-2\left(\kappa-\frac{2}{3} \eta\right)(\boldsymbol{n} \cdot \boldsymbol{u}) \boldsymbol{I}-2 \eta(\boldsymbol{u} \boldsymbol{n}+\boldsymbol{n u})\right] \mathrm{d} S
$$

where where $\boldsymbol{n}$ is the normal vector pointing outward on the particle surface, $\boldsymbol{\sigma}$ is the stress on the particle surface and $\boldsymbol{u}$ is the fluid velocity. The particle stresslet is the symmetric part of the first moment of the surface stress on the particle. The antisymmetric part of the first moment of the stress constitutes the torque acting on the particle. The total hydrodynamic stresslet for a particle is the sum of the contributions from the imposed rate-of-strain, Brownian motion of the particles and inter-particle forces $\left(S^{H}=S^{E}+S^{B}+S^{P}\right)$.

The particle stresslets can be expressed in terms of hydrodynamic resistance functions as

$$
\begin{aligned}
& \boldsymbol{S}^{B}=-k T \boldsymbol{\nabla} \cdot \boldsymbol{R}_{S U} \cdot \boldsymbol{R}_{F U}^{-1}, \\
& \boldsymbol{S}^{P}=-\left(\boldsymbol{R}_{S U} \cdot \boldsymbol{R}_{F U}^{-1}+\boldsymbol{x} \boldsymbol{I}\right) \cdot \boldsymbol{F}^{P}, \\
& \boldsymbol{S}^{E}=-\left(\boldsymbol{R}_{S U} \cdot \boldsymbol{R}_{F U}^{-1} \cdot \boldsymbol{R}_{F E}-\boldsymbol{R}_{S E}\right):\langle\boldsymbol{e}\rangle,
\end{aligned}
$$

where the derivative is with respect to the last index of the inverse of the resistance matrix $\left(\boldsymbol{R}_{F U}^{-1}\right)$ and $\boldsymbol{F}^{P}$ is the colloidal interparticle force. Here $\boldsymbol{R}_{F U}(\boldsymbol{x})$ and $\boldsymbol{R}_{F E}(\boldsymbol{x})$ are the configuration-dependent resistance tensors that give the hydrodynamic force/torques on the particles due to their motion relative to the fluid and owing to the imposed flow respectively. The stresslets $S^{B}, S^{P}$ and $S^{E}$ denote a matrix of all the particle stresslets. The vector $\boldsymbol{x}$ denotes the configuration - position and orientation — of the particles. The tensors $\boldsymbol{R}_{S U}$ and $\boldsymbol{R}_{S E}$ are similar to $\boldsymbol{R}_{F U}$ and $\boldsymbol{R}_{F E}$ and relate the particle stresslets to the particle velocity and the rate of strain. The combination of the resistance tensors is 
called the 'Grand Resistance Matrix':

$$
\mathcal{R}=\left[\begin{array}{ll}
\boldsymbol{R}_{F U} & \boldsymbol{R}_{F E} \\
\boldsymbol{R}_{S U} & \boldsymbol{R}_{S E}
\end{array}\right] .
$$

For a given set of particle velocities $\boldsymbol{U}^{\prime}$ relative to the surrounding fluid, and fluid rate-of-strain $\boldsymbol{E}^{\infty}$, the forces, torques and stresslets for all the particles can be obtained from the Grand Resistance Matrix as

$$
\left[\begin{array}{l}
\boldsymbol{F} \\
\boldsymbol{S}
\end{array}\right]=-\mathcal{R} \cdot\left[\begin{array}{c}
\boldsymbol{U}^{\prime} \\
-\boldsymbol{E}^{\infty}
\end{array}\right],
$$

where $\boldsymbol{F}$ is the combined force-torque vector for all the particles. The rigid particles cannot deform with the fluid, hence the rate of deformation for the particles relative to the fluid is given by $-\boldsymbol{E}^{\infty}$. The particle velocities can be obtained using the grand resistance matrix if the forces and stresslets are known, and vice-versa.

In this study we restrict our analysis to hard sphere suspensions, so the inter-particle force is given only by the hard-sphere potential as

$$
\boldsymbol{F}^{P}=\frac{1}{2} \boldsymbol{n} \delta(r-2 a),
$$

where $\boldsymbol{n}$ is the normal vector along the line of centers of two touching particles and $\delta$ is the delta function at the surface of contact. With hydrodynamic interactions, a hard-sphere potential plays no dynamical role because the relative mobility of two particles coming into contact goes to zero and so the hard-sphere force causes no motion. The inter-particle force contribution to the bulk stress, $\boldsymbol{S}^{P}$ in (4.3c) is also zero because the $\boldsymbol{R}_{S U} \cdot \boldsymbol{R}_{F U}^{-1} \cdot \boldsymbol{F}^{P}$ term exactly cancels the $\boldsymbol{x} \boldsymbol{F}^{P}$ inter-particle stress. Instead, the hard-sphere nature of the particles is accounted for by the no-slip boundary condition on the surface of the particles and the Brownian contribution to the stress includes the hard-sphere collisional contribution to the macroscopic stress [Brady 1993b].

When the suspension is in equilibrium $(\langle\boldsymbol{e}\rangle \equiv 0)$ the bulk stress is given by

$$
\langle\boldsymbol{\Sigma}\rangle^{e q}=-\left(\left\langle p_{t h}\right\rangle_{f}^{e q}+\Pi\right) \boldsymbol{I},
$$


where $\Pi$ is the osmotic pressure:

$$
\Pi=n k T-\frac{1}{3} n\left[\left\langle S^{B}\right\rangle^{e q}+\left\langle S^{P}\right\rangle^{e q}\right]
$$

and $S$ denotes the trace of the corresponding stresslet, as in $\left\langle\boldsymbol{S}^{B}\right\rangle^{e q}=\frac{1}{3}\left\langle S^{B}\right\rangle^{e q} \boldsymbol{I}$. The superscript $e q$ denotes an average over the equilibrium distribution of the suspension microstructure. The effective bulk viscosity $\kappa_{e f f}$ relates the deviation of the trace of the bulk stress from its equilibrium value to the trace of the average rate-of-strain tensor:

$$
\frac{1}{3}\left(\boldsymbol{I}:\langle\boldsymbol{\Sigma}\rangle-\boldsymbol{I}:\langle\boldsymbol{\Sigma}\rangle^{e q}\right)=\kappa_{e f f} \frac{1}{3} \boldsymbol{I}:\langle\boldsymbol{e}\rangle,
$$

therefore $\kappa_{e f f}$ is given by

$$
\kappa_{e f f} \equiv \kappa+\left(-\left\langle p_{t h}\right\rangle_{f}+\left\langle p_{t h}\right\rangle_{f}^{e q}\right) /\langle e\rangle+\frac{1}{3} n\left[\left(\left\langle S^{B}\right\rangle-\left\langle S^{B}\right\rangle^{e q}\right)+\left(\left\langle S^{P}\right\rangle-\left\langle S^{P}\right\rangle^{e q}\right)+\left\langle S^{E}\right\rangle\right] /\langle e\rangle .
$$

Thus with knowledge of the instantaneous particle configuration and velocities we can find the forces and stresslets for all the particles, and from an ensemble average of the stresslets over a large number of configurations the suspension bulk viscosity can be determined.

\subsection{Stokesian Dynamics}

Simulation of a particulate suspensions in Stokes flow poses at least two main problems. The first is the determination of the many-body long-range interactions among particles. The disturbance in the fluid velocity due to the motion of a particle decays slowly as $1 / r$, where $r$ is the distance from the particle, resulting in many-body hydrodynamic interactions between particles. Therefore calculation of the particle interactions cannot be truncated to a fixed range and no simple pairwise-additive approximation can be made. In addition, the force required to move two particles relative to each other increases in inverse proportion to the separation distance as the viscous fluid has to squeeze through a narrow gap between them (lubrication effect), causing the particles to slow down considerably at higher concentrations. The presence of lubrication forces makes conventional numerical techniques (such as the boundary-integral technique) very computationally expensive because the 
flow near particle surfaces would have to be resolved in more detail as particles approach each other. The second problem is the determination of the spatial distribution of particles (the microstructure) and how it changes with time in response to any external forcing and due to Brownian motion. In a dynamic process in which the particles undergo relative motion as in a shear flow for example, the distribution of particles cannot be specified a priori but must be solved for as part of the problem.

\subsubsection{Review of the existing method}

The Stokesian Dynamics (SD) technique developed by Durlofsky, Brady and Bossis [1987] successfully accounts for both the many-body interactions and the near-field lubrication forces by splitting the hydrodynamics into a far-field mobility calculation and a pairwise additive resistance calculation. This method was extended to infinite periodic suspensions [Phillips et al. 1988; Brady and Bossis 1988] and has been used successfully to give accurate results for many problems where the system size is of relatively little importance. Following is a brief overview of Stokesian Dynamics.

For $N$ rigid particles suspended in a Newtonian fluid of viscosity $\eta$ and density $\rho$, the motion of the fluid is governed by the Navier-Stokes equations, while the motion of the particles is described by the coupled $N$-body equation of motion:

$$
\boldsymbol{m} \cdot \frac{\mathrm{d} \boldsymbol{U}_{p}}{\mathrm{~d} t}=\boldsymbol{F}^{h}+\boldsymbol{F}^{p}
$$

which simply states that the mass times the acceleration equals the sum of the forces. In (4.11), $\boldsymbol{m}$ is the generalized mass/moment-of-inertia matrix of dimensions $6 N \times 6 N, \boldsymbol{U}_{p}$ is the particle translational/rotational velocity vector of dimension $6 N$, and $\boldsymbol{F}^{h}$ and $\boldsymbol{F}^{p}$ are the hydrodynamic and external force-torque vectors acting on the particles, also of dimension $6 \mathrm{~N}$ each. When the motion on the particle scale is such that the particle Reynolds number is small, the fluid equations of motion becomes linear (Stokes equation) and the hydrodynamic forces and torques acting on the particles in a bulk linear flow can be computed directly from the instantaneous particle configuration:

$$
\boldsymbol{F}^{h}=-\boldsymbol{R}_{F U} \cdot\left(\boldsymbol{U}_{p}-\boldsymbol{u}^{\infty}\right)+\boldsymbol{R}_{F E}: \boldsymbol{E}^{\infty}
$$

Here, $\boldsymbol{u}^{\infty}$ is the translational/rotational velocity of the bulk linear flow evaluated at the particle center 
and $\boldsymbol{E}^{\infty}$ is the externally imposed rate-of-strain tensor.

The long-range interactions are computed by expanding the force density on the surface of each particle in a series of moments about the center of the particle. The zeroth moment is simply the net force acting on a particle (plus a potential dipole for spherical particles), the first moment can be decomposed into the torque and the stresslet, while higher moments are neglected. This level of truncation gives the minimum set of unknowns per particle required in a bulk linear flow and has been shown to give very accurate results for many hydrodynamic problems. The relation between the far-field forces and stresslets and the far-field contribution to the particle velocities is given succinctly by Faxén laws:

$$
\begin{gathered}
\boldsymbol{U}_{p}-\boldsymbol{u}^{\infty}(\boldsymbol{x})=-\frac{1}{6 \pi \eta a} \boldsymbol{F}+\left(1+\frac{a^{2}}{6} \boldsymbol{\nabla}^{2}\right) \boldsymbol{u}_{f f}, \\
\boldsymbol{\Omega}_{p}-\omega^{\infty}(\boldsymbol{x})=-\frac{1}{8 \pi \eta a^{3}} \boldsymbol{T}+\frac{1}{2} \boldsymbol{\nabla} \times \boldsymbol{u}_{f f} \\
-\boldsymbol{E}^{\infty}=-\frac{3}{20 \pi \eta a} \boldsymbol{S}+\left(1+\frac{a^{2}}{10} \boldsymbol{\nabla}^{2}\right) \boldsymbol{e}_{f f},
\end{gathered}
$$

where the subscript $f f$ stands for far-field. In the original implementation [Phung 1992] the particle stresslets have 5 independent components corresponding to each particle and therefore are implemented as a vector of size $5 N$ in the SD algorithm. The imposed rate of strain $\boldsymbol{E}^{\infty}$ also has 5 independent components and is implemented as a vector of size $5 N$. With the inclusion of expansion flow the rate-of-strain tensor has an additional independent component given by the rate of expansion, so it is implemented as a vector of size $6 N$ with 6 components for each particle. There must also be a corresponding entry for the pressure moment for each prticle, defined as the trace of the stresslet tensor. Therefore the stresslets will also be implemented with a total of $6 \mathrm{~N}$ independent components.

The inverse of the grand resistance matrix, known as the grand mobility matrix $\left(\mathcal{M}^{\infty}\right)$ relates the particle velocities and rate-of-strain relative to the bulk suspension velocity and rate-of-strain respectively, to the force and stresslet acting on each particle. In an infinite suspension the velocity field that would be present at any point in the suspension in the absence of the disturbance flows due to the presence of a particle at that point, is given by the spatial average of the velocity field over the entire suspension (the bulk suspension velocity). The mobility matrix is constructed in pairwise 
additive fashion from the Faxén laws for each particle pair and is simply a restatement of the Faxén formulae for all the particles in matrix form. The mobility interactions are summed over an infinite periodic lattice of the particle configuration using the Ewald summation technique as described by Beenakker [1986]. Upon inversion of $\mathcal{M}^{\infty}$ infinite reflections among all the moments and all particles are computed [Durlofsky et al. 1987] and the far-field resistance matrix thus obtained contains the true many-body interactions. The near-field lubrication interactions, which would only be reproduced in $\left(\mathcal{M}^{\infty}\right)^{-1}$ if all multipole moments were included, are added to the resistance matrix in a pairwise additive fashion to complete the grand resistance matrix:

$$
\mathcal{R}=\left(\mathcal{M}^{\infty}\right)^{-1}+\mathcal{R}_{2 B}-\mathcal{R}_{2 B}^{\infty},
$$

where $\mathcal{R}_{2 B}$ is the matrix of exact two-body resistance interactions [Jeffrey and Onishi 1984] and $\mathcal{R}_{2 B}^{\infty}$ is the far-field contribution to the pair interactions from the inversion of the mobility matrix.

An evolution equation for the particle configuration is obtained by integrating (4.11) twice over a time step $\Delta t$ larger than the inertial relaxation time $\tau_{b}=m / 6 \pi \eta_{0} a$ but small compared with the time over which the configuration changes leading to particle displacements given as

$$
\Delta \boldsymbol{x}=\left\{\boldsymbol{u}^{\infty}+\boldsymbol{R}_{F U}^{-1} \cdot\left[\boldsymbol{R}_{F E}: \boldsymbol{E}^{\infty}+\boldsymbol{F}^{p}\right]\right\} \Delta t+k T \boldsymbol{\nabla} \cdot \boldsymbol{R}_{F U}^{-1} \Delta t+\boldsymbol{X}(t)+O(\Delta t),
$$

where $\boldsymbol{X}(t)$ is the additional displacement due to Brownian motion, and $\left(k T \boldsymbol{\nabla} \cdot \boldsymbol{R}_{F U}^{-1} \Delta t\right)$ is a deterministic displacement from the configurational-space divergence of the $N$-particle diffusivity. Thus at each time step the disturbance force on each particle due to the presence of other particles in the imposed flow is calculated and then used to determine the disturbance velocity for each particle. The displacement due to Brownian motion is added as described later, and the particle stresslets are calculated from (4.3a), (4.3b) and (4.3c). The particle positions are updated to get the new configuration and the process is repeated. For more details the reader is referred to the doctoral thesis of Phung [1992] who implemented the SD algorithm in FORTRAN. The same code was modified for the current study. In an expansion/compression flow the size of the simulation cell must also be adjusted at the same rate at which the particle are coming closer so that the particle images also move closer at the same rate and the homogeneity of the infinite suspension is maintained. 


\subsubsection{Linear compressible flow}

Previously, Stokesian Dynamics has been used to simulate particles in incompressible flow situations. In order to simulate linear compressible flows, the bulk rate of compression or expansion given by the trace of the rate-of-strain tensor $\boldsymbol{E}^{\infty}$ must also be included. The pressure moment, defined as the trace of the first moment of the stress, is an additional unknown that must also be computed as part of the simulation. Jeffrey, Morris, and Brady [1993] calculated the two-body hydrodynamic resistance functions for the pressure moment in an incompressible linear flow. For a given pair of particles labelled 1 and 2 the pressure moment is related to the particle velocities and their rate-of-strain as

$$
\left(\begin{array}{c}
S_{1}^{H} \\
S_{2}^{H}
\end{array}\right)=-\eta\left(\begin{array}{llll}
\boldsymbol{P}_{11} & \boldsymbol{P}_{12} & \boldsymbol{Q}_{11} & \boldsymbol{Q}_{12} \\
\boldsymbol{P}_{21} & \boldsymbol{P}_{22} & \boldsymbol{Q}_{21} & \boldsymbol{Q}_{22}
\end{array}\right) \cdot\left(\begin{array}{c}
\boldsymbol{U}_{1}-\boldsymbol{U}^{\infty} \\
\boldsymbol{U}_{2}-\boldsymbol{U}^{\infty} \\
-\boldsymbol{E}^{\infty} \\
-\boldsymbol{E}^{\infty}
\end{array}\right)
$$

where $\boldsymbol{P}_{\alpha \beta}=\pi\left(a_{\alpha}+a_{\beta}\right)^{2} X_{\alpha \beta}^{P} \boldsymbol{d}$ relates the pressure moment to the particle velocities. The rigid particles cannot deform so tha particle rate-of-strain is zero. The $X_{\alpha \beta}^{P}$ are hydrodynamic resistance functions and $\boldsymbol{d}=\left(\boldsymbol{x}_{2}-\boldsymbol{x}_{1}\right) /\left|\boldsymbol{x}_{2}-\boldsymbol{x}_{1}\right|$. The analogous expression for the tensors relating the pressure moment to an imposed linear traceless shear flow was given as $\boldsymbol{Q}_{\alpha \beta}=\pi\left(a_{\alpha}+a_{\beta}\right)^{3} X_{\alpha \beta}^{Q}\left[\boldsymbol{d} \boldsymbol{d}-\frac{1}{3} \boldsymbol{I}\right]$ , where the $X_{\alpha \beta}^{Q}$ are also hydrodynamic resistance functions. These functions can also be used to compute the induced force and deviatoric stresslet in a compressible linear flow by virtue of the reciprocal relation $\left(\boldsymbol{R}_{F E}^{\dagger}=\boldsymbol{R}_{S U}\right)$ :

$$
\left(\begin{array}{c}
\boldsymbol{F}_{1}^{H} \\
\boldsymbol{F}_{2}^{H} \\
\boldsymbol{S}_{1}^{H} \\
\boldsymbol{S}_{2}^{H}
\end{array}\right)=-\eta\left(\begin{array}{ll}
\boldsymbol{P}_{11} & \boldsymbol{P}_{12} \\
\boldsymbol{P}_{21} & \boldsymbol{P}_{22} \\
\boldsymbol{Q}_{11} & \boldsymbol{Q}_{12} \\
\boldsymbol{Q}_{21} & \boldsymbol{Q}_{22}
\end{array}\right) \cdot\left(\begin{array}{c}
-E_{k k}^{\infty} \\
-E_{k k}^{\infty}
\end{array}\right)
$$

where $\boldsymbol{F}_{\alpha}^{H}$ and $\boldsymbol{S}_{\alpha}^{H}$ are the hydrodynamic force and stresslet for particle $\alpha$, and $E_{k k}^{\infty}$ is the trace of the imposed rate-of-strain which is the bulk rate of expansion. The two-particle hydrodynamic resistance functions for computing the pressure moment for particles due to an imposed compressible 
flow were computed by Khair et al. [2006] and designated as $T_{\alpha \beta}^{Q}$, so that the complete $\boldsymbol{Q}_{\alpha \beta}$ function becomes

$$
\boldsymbol{Q}_{\alpha \beta}=\pi\left(a_{\alpha}+a_{\beta}\right)^{3}\left[X_{\alpha \beta}^{Q}\left(\boldsymbol{d} \boldsymbol{d}-\frac{1}{3} \boldsymbol{I}\right)+T_{\alpha \beta}^{Q} \frac{1}{3} \boldsymbol{I}\right]
$$

such that the trace of $\boldsymbol{Q}_{\alpha \beta}$ is no longer zero by design. These new resistance functions are added to $\boldsymbol{R}^{S U}$ and $\boldsymbol{R}^{F E}$ in the two-body lubrication part of the Grand Resistance Matrix (4.14). The particle stresslets now have six independent components and are therefore implemented as a vector of size $6 \mathrm{~N}$, where the sixth component for each particle is the pressure moment, or trace of the stresslet. The rate-of-strain tensor too has six independent components and is also implemented as a vector of size $6 N$, with the sixth component given by $\frac{1}{3} E_{k k}^{\infty}$ for each particle.

The Faxén law for computing the pressure moment in a linear compressible flow is given by

$$
S_{k k}^{H}=-\frac{16}{3} \pi \eta a^{3} E_{k k}^{\infty}+4 \pi a^{3} p^{s}
$$

where $S_{k k}^{H}$ is the trace of the stresslet (pressure moment), $E_{k k}^{\infty}$ is the trace of the rate-of-strain tensor, and $p^{s}=p^{U}+p^{E}$ is the induced Stokes flow pressure due to the imposed particle velocities $\left(p^{U}\right)$ and rate-of-strain $\left(p^{E}\right)$, including all reflected hydrodynamic interactions. The induced pressure $p^{s}$ is simply the disturbance pressure associated with the disturbance Stokes flow $\boldsymbol{u}^{s}$ generated due to the finite size of the particles and they are related as

$$
p^{s}=2 \eta \frac{\boldsymbol{u}^{s} \cdot \boldsymbol{x}}{r^{2}}
$$

where $\boldsymbol{x}$ is the position vector in the same frame of reference as $\boldsymbol{u}^{s}$. Equation (4.19) along with (4.13a), (4.13b) and (4.13c) completes the set of equations needed to compute the mobility matrix including the fluid expansion and the pressure moment. Note that the calculation of the pressure moment does not require the calculation of any additional reflected interactions, only the fluid pressure associated with all existing reflected flows needs to be computed and that is given by the $X_{\alpha \beta}^{P}$ and $X_{\alpha \beta}^{Q}$ resistance functions for particle pairs. The only reflected hydrodynamic interactions that were not included previously are due to the linear compression $E_{k k}^{\infty}$, and are given by the $T_{\alpha \beta}^{Q}$ resistance functions which are now included in the updated $\boldsymbol{R}_{S E}$ matrix. However, the two-body resistance functions only give the contribution to the pressure moment due to lubrication interactions between 
neighboring particles in a dense suspension.

\subsubsection{The Mobility matrix with expansion flow}

In order to compute the many-body far-field interactions we need to invert the pairwise mobility matrix obtained from Faxén laws, including the disturbance flow due to linear compression. The pair mobility functions are calculated using a truncated multipole expansion in combination with the Faxén laws and the Ewald summation technique is used to include long range interactions between infinite particles. Only the first-order disturbance due to a single particle in expansion flow given by

$$
\boldsymbol{u}_{i}=-\frac{1}{3} E_{k k}^{\infty} a^{3} \frac{\boldsymbol{x}_{i}}{r^{3}}
$$

needs to be included in the mobility matrix. The disturbance flow given by (4.21) from each particle is simply added to $\boldsymbol{u}_{f f}$ in (4.13a) and (4.13b) to get the far-field contribution to the particle velocities due to the imposed expansion flow. The velocity field in (4.21) is purely kinematic in nature as it simply accounts for the rigidity of the volume occupied by the particle in an otherwise expanding fluid and so there is no disturbance pressure directly associated with it. However it does affect the motion of the other particles which in turn generate secondary disturbance flows and the Stokes pressure related with these reflected flows contributes to the particle pressure moments. The reflected flows and the associated far-field forces, stresslets and pressure moments are computed upon inversion of the Grand Mobility Matrix $\left(\mathcal{M}^{\infty}\right)$.

The Faxén laws (14.3) are implemented in $\mathcal{M}^{\infty}$ in the form of mobility functions as described by Durlofsky et al. [1987]. The relevant mobility functions for expansion flow can be summarized as

$$
\left(\begin{array}{c}
\boldsymbol{U}_{1}-\boldsymbol{U}^{\infty} \\
\boldsymbol{U}_{2}-\boldsymbol{U}^{\infty} \\
-\boldsymbol{E}_{\infty} \\
-\boldsymbol{E}_{\infty}
\end{array}\right)=\left(\begin{array}{ll}
\boldsymbol{p}_{11} & \boldsymbol{p}_{12} \\
\boldsymbol{p}_{21} & \boldsymbol{p}_{22} \\
\boldsymbol{q}_{11} & \boldsymbol{q}_{12} \\
\boldsymbol{q}_{21} & \boldsymbol{q}_{22}
\end{array}\right) \cdot\left(\begin{array}{c}
S_{1}^{H} \\
S_{2}^{H}
\end{array}\right)
$$

where

$$
\boldsymbol{p}_{\alpha \beta}=\frac{1}{6 \pi \eta a^{2}} x_{\alpha \beta}^{p} \boldsymbol{d}
$$

relates the particle velocities to the particle pressure moments, and the corresponding hydrodynamic 
mobility functions $x_{\alpha \beta}^{p}$ are:

$$
x_{11}^{p}=x_{22}^{p}=0 \quad \text { and } \quad x_{12}^{p}=-x_{21}^{p}=-\frac{3}{8} \frac{1}{r^{2}} .
$$

Similarly, the the rate-of-strain tensor is related to the pressure moments by

$$
\boldsymbol{q}_{\alpha \beta}=\frac{1}{6 \pi \eta a^{3}}\left[x_{\alpha \beta}^{q}\left(\boldsymbol{d} \boldsymbol{d}-\frac{1}{3} \boldsymbol{I}\right)+t_{\alpha \beta}^{q} \boldsymbol{I}\right]
$$

with the relevant hydrodynamic mobility functions given by

$$
x_{11}^{q}=x_{22}^{q}=0 \quad \text { and } \quad x_{12}^{q}=x_{21}^{q}=-\frac{9}{8} \frac{1}{r^{2}}
$$

and

$$
t_{11}^{q}=t_{22}^{q}=\frac{3}{8} \quad \text { and } \quad t_{12}^{q}=t_{21}^{q}=0
$$

Since no reflected flows are included in the construction of the mobility matrix, the mobility functions listed above only give the particle velocities and rate-of-strain due to the disturbance velocity given by (4.21) arising from the rate of expansion. Thus, the pressure moment in (4.22) contains only the single-particle self-contribution for each particle corresponding to the fluid disturbance velocity in (4.21). The remaining far-field contribution to the pressure moment will be computed from the Stokes pressure corresponding to all the reflected disturbance flows upon inversion of the mobility matrix. The mobility matrix is constructed pair-wise for all the particles and long-range interactions in an infinite system are evaluted using the Ewald summation technique. This is accomplished for the mobility functions in (4.22) by noting that

$$
-\frac{1}{2} \nabla \nabla^{2} r=\frac{d}{r^{2}}
$$

and proceeding as in Beenakker [1986] but with $\nabla \nabla^{2} r$ instead of the Rotne-Prager tensor.

As a corollary $\left(\mathcal{M}^{\infty}\right)$ is no longer symmetric because there is no compressible disturbance flow that could be generated by the force or the traceless stresslet acting on a particle. Specifically, the 
transpose of the portion of the mobility matrix covered by (4.22) can be written as:

$$
\left(\begin{array}{c}
-E_{k k}^{\infty} \\
-E_{k k}^{\infty}
\end{array}\right)=\frac{1}{6 \pi \eta a^{3}}\left(\begin{array}{cccc}
0 & 0 & t_{11}^{q} \boldsymbol{I} & 0 \\
0 & 0 & 0 & t_{22}^{q} \boldsymbol{I}
\end{array}\right) \cdot\left(\begin{array}{c}
\boldsymbol{F}_{1}^{H} \\
\boldsymbol{F}_{2}^{H} \\
\boldsymbol{S}_{1}^{H} \\
\boldsymbol{S}_{2}^{H}
\end{array}\right)
$$

This asymmetry arises because of the very nature of the pressure moment, in that it is simply a measurement of the Stokes pressure associated with disturbance flows that have already been accounted for except those resulting from expansion. Therefore we may add the pressure moment in the mobility calculations but repeating any disturbance flows in the matrix to make it symmetric would be physically incorrect. An imposed linear compressible flow leads to an incompressible disturbance flow in the fluid, but an incompressible imposed flow does not cause any compressible disturbance flow. Therefore the mobility matrix is no longer symmetric. Mathematically, this ensures that upon inversion of the mobility matrix all the reflected interactions between particles are computed, including those arising from compression, but no spurious interactions are computed for the pressure moment. The $\boldsymbol{R}_{S E}$ part of the far-field resistance matrix thus obtained does not contain the hydrodynamic resistance functions for calculating the pressure moment due to the deviatoric rate-of-strain. However, these are the same functions as the ones for calculating the contribution to the deviatoric stresslet due to linear compression present in $\boldsymbol{R}^{S E}$, which are copied over to make the $\boldsymbol{R}_{S E}$ matrix symmetric. Similarly, the far field contribution to the $\boldsymbol{R}^{F E}$ matrix arising from $E_{k k}^{\infty}$ must be copied over to get the far-field contribution for the pressure moment in the $\boldsymbol{R}^{S U}$ matrix. With these modifications the SD technique can be used for modeling of compression flows in suspensions.

\subsubsection{Brownian motion}

In addition to the imposed flow, particles in suspension also undergo Brownian motion due to thermal fluctuations in the surrounding fluid. This is modeled in Stokesian Dynamics by computing a new set of Brownian forces acting on the particles at each time step [Brady and Bossis 1988]. The Brownian force vector $\boldsymbol{F}^{b}$ is a Gaussian stochastic variable obeying the following statistics:

$$
\overline{\boldsymbol{F}^{b}}=0, \quad \overline{\boldsymbol{F}^{b}(0) \boldsymbol{F}^{b}(t)}=2 k T \boldsymbol{R}_{F U} \delta(t),
$$


where the over-bar denotes an ensemble average over the thermal fluctuations in the fluid, $k$ is Boltzmann's constant, $T$ is the absolute temperature, and $\delta(t)$ is the delta function. The correlation at 0 and $t$ is a consequence of the fluctuation-dissipation theorem for the $N$-particle system. The Brownian forces are calculated as

$$
\boldsymbol{F}^{b}(t)=\sqrt{2 k T} \boldsymbol{A} \cdot \boldsymbol{\Psi}(t)
$$

with

$$
\boldsymbol{A} \cdot \boldsymbol{A}^{T}=\boldsymbol{R}_{F U}
$$

where $\boldsymbol{\Psi}(t)$ is a normal deviate $(\overline{\boldsymbol{\Psi}(t)}=0$ and $\overline{\boldsymbol{\Psi}(t) \boldsymbol{\Psi}(t)}=1)$. The corresponding Brownian displacements $\boldsymbol{X}(t)$ can be obtained by solving the system of linear equations given by

$$
\boldsymbol{A}^{T} \cdot \boldsymbol{X}(t)=\sqrt{2 k T} \Delta t \boldsymbol{\Psi}(t)
$$

The divergence of $\boldsymbol{R}_{F U}^{-1}$ in the mean drift term $\left(k T \boldsymbol{\nabla} \cdot \boldsymbol{R}_{F U}^{-1} \Delta t\right)$ is evaluated numerically by using a modified mid-point scheme as described in [Banchio and Brady 2003]. A similar scheme is used to calculate the Brownian stresses, which also include a divergence in their expressions (4.3c). The particle configuration is evolved by a fraction of the time step ( $\Delta t / n$ with $n$ typically on the order of 100) and the $\boldsymbol{R}_{F U}^{-1}$ and $\boldsymbol{R}_{S U}$ matrices are evaluated again with this configuration. The small step is used to avoid particle overlaps in the intermediate configuration. The divergences can now be calculated from the difference in the resistance matrices over the fractional time step. The Brownian contribution to the pressure moment is computed simply by using the updated $\boldsymbol{R}^{S U}$ matrix which includes the $X_{\alpha \beta}^{P}$ resistance functions in the Brownian stress calculation and the far-field component obtained from inversion of the mobility matrix.

\subsection{Accelerated Stokesian Dynamics}

The direct solution of (4.11) as implemented in the Stokesian Dynamics method is computationally expensive since it involves the costly $O\left(N^{2}\right)$ calculation of the far-field mobility matrix and its costly $O\left(N^{3}\right)$ inversion. This limits the simulation to $N$ of the order of a hundred, where $N$ is the number 
of particles in a unit cell with an infinite periodic array of cells. The two-body near-field resistance matrices are stored in a sparse form by including only the nonzero contributions from neighboring particles within a cutoff distance, thus allowing the construction of the sparse $\mathcal{R}_{2 B}-\mathcal{R}_{2 B}^{\infty}$ matrix in $O(N)$ operations. The speed-limiting part is therefore the construction and inversion of the mobility matrix.

An alternate approach to the SD method called Accelerated Stokesian Dynamics (ASD) was developed by Sierou and Brady [2001] and reduces the computational cost to $O(N \log N)$ operations. In this approach the far-field forces are computed directly using an iterative procedure, thus foregoing the need to even construct the complete mobility matrix $\left(\mathcal{M}^{\infty}\right)$ because only the product of the matrix with a vector is required in the inversion procedure. The hydrodynamic force in (4.11) is split into a far-field and a near-field part as

$$
\boldsymbol{F}^{h}=\boldsymbol{F}_{f f}^{h}-\boldsymbol{R}_{F U, n f} \cdot\left(\boldsymbol{U}_{p}-\boldsymbol{u}^{\infty}\right)+\boldsymbol{R}_{F E, n f}: \boldsymbol{E},
$$

where the subscript $f f$ denotes far-field and $n f$ denotes near-field. The near-field resistance matrix in (4.33) corresponds simply to the relevant component of $\mathcal{R}_{2 B}-\mathcal{R}_{2 B}^{\infty}$ in (4.14). The particle velocities $\left(\boldsymbol{U}_{p}-\boldsymbol{u}^{\infty}\right)$ can be taken from the previous time step or computed as a nested iterative procedure.

The far-field forces (and stresslets) for each particle are computed as an iterative procedure starting with an initial guess, for which the forces in the previous time step may be used. The force and stresslet acting on each particle is distributed over a uniform 3D grid as a set of point forces using a particle-mesh (PME) technique [Hockney and Eastwood 1988]. This allows the use of Hasimoto's solution [Hasimoto 1959] of the Stokes equation for flow past a periodic array of spheres to calculate the disturbance velocity in the fluid due to the point forces on the mesh nodes. This is equivalent to calculating the far-field disturbance velocity due to the forces acting on the particles. Next, the disturbance velocity at the center of each particle is found by interpolating from the uniform grid and the Faxén laws are used to calculate the forces and stresslets on the particles. This procedure is repeated until the forces converge. The new particle velocities are then found from (4.33) by iterative inversion of the sparse $\boldsymbol{R}_{F U, n f}$ matrix. This can be accomplished in $O(N)$ operations by making use of sparse solvers for which only the $O(N)$ multiplication of the resistance matrix with a vector is required. 
The advantage of using this technique is that Hasimoto's solution for a periodic array of point forces involves splitting the velocity field calculation into a wave-space part that can be computed using Fast Fourier Transform (FFT) techniques, and a short-range real-space part that can be computed in $O(N)$ operations, based on a splitting parameter $\alpha$. The wave-space contribution is calculated in $O\left(N_{m}^{3} \log N_{m}\right)$ operations where $N_{m}$ is the total number of grid points in each direction. An optimum value of $\alpha$ and $N_{m}$ can be found that will give reasonable accuracy and keep the computational cost down to $O(N \log N)$ operations. Using this method periodic systems with $N$ of the order of a thousand can be simulated on desktop workstations in a reasonable time with good accuracy.

\subsubsection{Expansion flow in ASD}

The far-field disturbance velocity due to the presence of rigid particles in an expanding fluid can be modeled simply by treating each particle as a point fluid sink with strength $S=\frac{4}{3} \pi a^{3} E$, where $E$ is the rate of expansion in the fluid and $a$ is the radius of the particles. The mass conservation equation for the disturbance fluid velocity is now

$$
\nabla \cdot \boldsymbol{u}=-\sum_{n} S^{n} \delta\left(\boldsymbol{x}-\boldsymbol{x}_{n}\right)
$$

where the summation is over all the particles and $\boldsymbol{x}_{n}$ denotes the position vector for the center of particle $n$. One can show by solving (4.34) for a single particle that the disturbance velocity at a distance $r=a$ from the point sink is

$$
v=-\frac{1}{4 \pi r^{3}} S \boldsymbol{x}=-\frac{1}{3} E x \frac{a^{3}}{r^{3}}
$$

which is equal to the disturbance velocity due to the presence of a rigid particle of radius $a$ in a fluid expanding at rate $E$, given in (4.21). Note that in the absence of the particle the expanding fluid would be equivalent to a continuous fluid source with density $E$ in the volume occupied by the particle. Therefore treating the particles as a fluid source of strength $S$ essentially cancels out the expanding fluid in the space it is occupying and thus the rigid nature of the particle is ensured.

Each point source is distributed over the PME mesh preserving the total source strength, as is done for the force acting on a particle in ASD. The far-field disturbance velocity in the fluid due 
to the point sources on the grid is calculated using Hasimoto's technique by splitting the velocity field calculation into a wave-space part that can be computed using Fast Fourier Transform (FFT) techniques, and a short-range real-space part. Expressions for the wave-space and real-space contributions are derived in the appendix to this chapter. The fluid velocity due to the particle sources is added to the velocity contribution from the forces and stresslets acting on the particles and interpolated to find the far-field particle velocities using Faxén laws. Calculation of the pressure moment is not required for determining the disturbance flows. This is equivalent to the asymmetric nature

of the mobility matrix in SD. The pressure moment is evaluated after the fact from the converged particle forces and stresslets after the iterative inversion is complete. Calculation of the far-field pressure moment was already implemented in ASD and expressions for the wave-space and realspace contribution are given in the doctoral thesis of Sierou [2002]. The near-field contribution to the pressure moment is computed from the hydrodynamic resistance functions as in the SD method.

\subsection{Conclusions}

We have described the changes made to the Stokesian Dynamics and Accelerated Stokesian Dynamics techniques to enable simulation of compressible flows in suspensions. The updated simulation methods have enabled the study of a larger variety of suspension flows where the particle phase may undergo expansion or compression either by changing the number density of particles, or even having the particles themselves expand or contract in addition to any other imposed forcing. The changes can simply be added onto the existing algorithm and do not require extensive rewriting of the code. The only portion that needs attention is the inversion of the mobility matrix in SD as it is no longer symmetric with the inclusion of the pressure moment. This work also points out that although the resistance matrix must necessarily be symmetric because it represents the dissipation of energy by the viscous flows the mobility matrix need not be symmetric because it is primarily a mathematical construct that enables the computation of many-body interactions in a straightforward manner. 


\section{Appendix B - Far-field velocity calculation for particles in an expan- sion flow}

Each particle can be treated as a point sink with strength $S=\frac{4}{3} \pi a^{3} E$, where $E$ is the rate of expansion in the fluid, and $a$ is the radius of the particle. For a single particle in expansion flow, the fluid conservation equation for the disturbance flow can be written as:

$$
\nabla \cdot v=-S \delta\left(x-x_{0}\right)
$$

where $x_{0}$ is the center of the particle. Since the only forcing in the problem is a scalar, the velocity must be of the form $\boldsymbol{v}=f(r) S \boldsymbol{x}$. From the conservation of mass in a sphere of radius $r$ centered at $x_{0}$ we get

$$
\begin{gathered}
\int_{\partial S} \boldsymbol{n} \cdot \boldsymbol{v} \mathrm{d} S=\int_{V_{0}} \nabla \cdot \boldsymbol{v} \mathrm{d} V=-S \\
f(r)=-\frac{1}{4 \pi r^{3}} \\
\boldsymbol{v}=-\frac{1}{4 \pi r^{3}} S \boldsymbol{x}=-\frac{1}{3} E \boldsymbol{x} \frac{a^{3}}{r^{3}} .
\end{gathered}
$$

This is the known result for the disturbance flow around a particle in an expanding fluid. Now consider a collection of $N$ particles repeated in a periodic array. Again, each particle is represented as a point sink with strength $S=\frac{4}{3} \pi a^{3} E$. The conservation equation for the disturbance flow is now

$$
\nabla \cdot v=-\sum_{n} S^{n} \delta\left(x-x_{n}\right)
$$

where the summation is over all the particles. Doing a Fourier transform over the cell volume, this becomes

$$
-2 \pi i \boldsymbol{k} \cdot \hat{\boldsymbol{v}}_{\boldsymbol{k}}=-\frac{1}{V_{0}} \hat{S}_{\boldsymbol{k}}
$$

where $\hat{S}_{k}$ is the finite Fourier transform of all the point sinks and $V_{0}$ is the cell volume. The source strength for each particle is distributed over the PME grid preserving the total strength and average location of the particle center. Therefore we don't assume $S$ to be constant for all the particles, and 
so we work with the FFT of $S$. The solution for the velocity is given by

$$
\begin{gathered}
\hat{\boldsymbol{v}}_{\boldsymbol{k}}=-\frac{i \boldsymbol{k} \hat{S}_{\boldsymbol{k}}}{2 \pi V_{0} k^{2}}, \quad \boldsymbol{k} \neq 0 \\
\boldsymbol{v}=\boldsymbol{v}_{0}+\sum_{\boldsymbol{k} \neq 0} \hat{\boldsymbol{v}}_{\boldsymbol{k}} e^{-2 \pi i \boldsymbol{k} \cdot \boldsymbol{x}}=\boldsymbol{v}_{0}-\frac{i}{2 \pi V_{0}} \sum_{\boldsymbol{k} \neq 0} \hat{S}_{\boldsymbol{k}} \boldsymbol{k} \frac{e^{-2 \pi i \boldsymbol{k} \cdot \boldsymbol{x}}}{k^{2}},
\end{gathered}
$$

where $\boldsymbol{v}_{0}=0$ for the present case. We proceed as in Hasimoto [1959] to evaluate the summation.

$$
\begin{aligned}
\sigma_{m} & =\sum_{\boldsymbol{k} \neq 0} \frac{e^{-2 \pi i \boldsymbol{k} \cdot \boldsymbol{x}}}{k^{2 m}} \hat{S}_{\boldsymbol{k}} \\
& =\frac{\pi^{m}}{\Gamma(m)} \sum_{\boldsymbol{k} \neq 0} \int_{0}^{\infty} e^{-\pi k^{2} \beta-2 \pi i \boldsymbol{k} \cdot \boldsymbol{x}} \hat{S}_{\boldsymbol{k}} \beta^{m-1} \mathrm{~d} \beta \\
& =\frac{\pi^{m}}{\Gamma(m)} \int_{0}^{\infty} \beta^{m-1}\left[\sum_{\boldsymbol{k}} \hat{S}_{\boldsymbol{k}} e^{-\pi k^{2} \beta-2 \pi i \boldsymbol{k} \cdot \boldsymbol{x}}-\hat{S}_{0}\right] \mathrm{d} \beta
\end{aligned}
$$

A splitting parameter $\alpha$ is introduced and the integral is split into two parts, one from 0 to $\alpha$, and the other from $\alpha$ to $\infty$. Ewald's theta transformation formula

$$
\sum_{\boldsymbol{k}} \hat{S}_{\boldsymbol{k}} e^{-\pi k^{2} \beta-2 \pi i \boldsymbol{k} \cdot \boldsymbol{x}}=\frac{V_{0}}{\beta^{3 / 2}} \sum_{n} e^{-\pi\left(\boldsymbol{x}-\boldsymbol{x}_{n}\right) / \beta} S^{n}
$$

is then applied to the integral from 0 to $\alpha$. The general formula for the evaluation of $\sigma_{m}$ now becomes

$$
\begin{aligned}
\sigma_{m}=\frac{\pi^{m} \alpha^{m}}{\Gamma(m)}\left[V_{0} \alpha^{-3 / 2} \sum_{n} \phi_{-m+1 / 2}\left(\frac{\pi\left(\boldsymbol{x}-\boldsymbol{x}_{n}\right)^{2}}{\alpha}\right) S^{n}\right. & \left.-\frac{S^{n}}{m}\right] \\
& +\frac{\pi^{m} \alpha^{m}}{\Gamma(m)}\left[\sum_{\boldsymbol{k} \neq 0} \hat{S}_{\boldsymbol{k}} e^{-2 \pi i \boldsymbol{k} \cdot \boldsymbol{x}} \phi_{m-1}\left(\pi \alpha k^{2}\right)\right]
\end{aligned}
$$

where we have replaced $\beta=\alpha / \xi$ in the first integral and $\beta=\alpha \xi$ in the second, and the function $\phi_{v}$ is given by

$$
\phi_{v}(x)=\int_{1}^{\infty} \xi^{v} e^{-x \xi} \mathrm{d} \xi
$$


We need the derivative of $\sigma_{m}$ with respect to $\boldsymbol{x}$ in order to evaluate $\boldsymbol{v}$ :

$$
\begin{aligned}
\frac{\partial \sigma_{m}}{\partial x_{j}} & =-2 \pi i \sum_{\boldsymbol{k} \neq 0} k_{j} \frac{e^{-2 \pi i \boldsymbol{k} \cdot \boldsymbol{x}}}{k^{2 m}} \hat{S}_{\boldsymbol{k}} \\
& =-4 \pi^{2} V_{0}\left(\frac{i}{2 \pi V_{0}} \sum_{\boldsymbol{k} \neq 0} k_{j} \frac{e^{-2 \pi i \boldsymbol{k} \cdot \boldsymbol{x}}}{k^{2 m}} \hat{S}_{\boldsymbol{k}}\right) \\
v_{j} & =\frac{1}{4 \pi^{2} V_{0}} \frac{\partial \sigma_{m}}{\partial x_{j}}, \quad m=1 .
\end{aligned}
$$

Evaluating the derivative of $\sigma_{1}$ :

$$
\begin{gathered}
\sigma_{1}=\pi \alpha\left[V_{0} \alpha^{-3 / 2} \sum_{n} S^{n} \phi_{-1 / 2}\left(\frac{\pi\left(\boldsymbol{x}-\boldsymbol{x}_{n}\right)^{2}}{\alpha}\right)-1+\sum_{\boldsymbol{k} \neq 0} \hat{S}_{\boldsymbol{k}} e^{-2 \pi i \boldsymbol{k} \cdot \boldsymbol{x}} \phi_{0}\left(\pi \alpha k^{2}\right)\right] \\
\frac{\partial \sigma_{1}}{\partial x_{j}}=-\left[2 \pi^{2} \alpha^{-3 / 2} V_{0} \sum_{n} S^{n} \phi_{1 / 2}\left(\frac{\pi r^{2}}{\alpha}\right) x_{j}+2 \pi i \sum_{\boldsymbol{k} \neq 0} \hat{S}_{\boldsymbol{k}} k_{j} e^{-2 \pi i \boldsymbol{k} \cdot \boldsymbol{x}} \frac{e^{-\pi \alpha k^{2}}}{k^{2}}\right],
\end{gathered}
$$

where $x_{j}$ is shorthand for $x_{j}-x_{j}^{n}$ and $r=\left|\boldsymbol{x}-\boldsymbol{x}_{n}\right|$. The disturbance velocity can therefore be written as

$$
v_{j}=-\frac{\alpha^{-3 / 2}}{2} \sum_{n} S^{n} \phi_{1 / 2}\left(\frac{\pi r^{2}}{\alpha}\right) x_{j}-\sum_{\boldsymbol{k} \neq 0} \hat{S}_{\boldsymbol{k}} \frac{i k_{j}}{2 \pi V_{0} k^{2}} e^{-\pi \alpha k^{2}} e^{-2 \pi i \boldsymbol{k} \cdot \boldsymbol{x}}
$$

Substituting $S=\frac{4}{3} \pi a^{3} E$, we get

$$
v_{j}=-\sum_{n} \frac{E^{n}}{3} \frac{2 \pi}{\alpha^{3 / 2}} \phi_{1 / 2}\left(\frac{\pi r^{2}}{\alpha}\right) x_{j}-\sum_{\boldsymbol{k} \neq 0} \frac{\hat{E}_{\boldsymbol{k}}}{3} \frac{2 i k_{j}}{V_{0} k^{2}} e^{-\pi \alpha k^{2}} e^{-2 \pi i \boldsymbol{k} \cdot \boldsymbol{x}},
$$

where $\hat{E}_{\boldsymbol{k}}$ is the finite Fourier transform of the expansion rate $E$ distributed over a regular grid near the center of each particle, and $a$ is used to non-dimensionalize $\alpha$ and $V_{0}$ in the two summations, respectively. 


\section{Chapter 5}

\section{Equilibrium Properties via Simulation}

\subsection{Introduction}

Several transport properties of particle systems in the regime of small deviations from equilibrium can be deduced from knowledge of the particle microstructure and fluctuations in the microstructure at equilibrium. At high particle concentrations the simple closures used for the dilute theory are no longer applicable and one must resort to numerical simulations to account for interactions between multiple particles. In this chapter we describe the Brownian Dynamics, Stokesian Dynamics and Accelerated Stokesian Dynamics simulations performed for Brownian particles at equilibrium and the calculation of the suspension bulk viscosity from simulations.

\subsection{Suspension stress and the bulk viscosity}

The average bulk stress in a suspension can be written as [Brady 1993a]

$$
\langle\boldsymbol{\Sigma}\rangle=-\left\langle p_{t h}\right\rangle_{f} \boldsymbol{I}+2 \eta\langle\boldsymbol{e}\rangle+\left(\kappa-\frac{2}{3} \eta\right)\langle\boldsymbol{\nabla} \cdot \boldsymbol{u}\rangle \boldsymbol{I}+\left\langle\boldsymbol{\Sigma}_{p}\right\rangle,
$$

where $\boldsymbol{e}$ is the rate of strain in the fluid, $\boldsymbol{I}$ is the isotropic tensor and $\left\langle p_{t h}\right\rangle$ is the thermal pressure of the fluid. The angle brackets $\langle\ldots\rangle$ denotes an average over the entire suspension (particles plus fluid), and $\langle\ldots\rangle_{f}$ denotes an average over the fluid phase only. The trace of $\langle\boldsymbol{e}\rangle$, given by $\langle e\rangle \equiv\langle\boldsymbol{\nabla} \cdot \boldsymbol{u}\rangle$ is the average rate of expansion of the suspension and the contribution to the isotropic stress due to fluid expansion is $\kappa\langle\boldsymbol{\nabla} \cdot \boldsymbol{u}\rangle$ with $\kappa$ the bulk viscosity of the pure fluid. The deviatoric stress contribution

from the fluid is $2 \eta\langle\boldsymbol{e}\rangle$ where $\eta$ is the fluid shear viscosity. The particle-phase stress $\left\langle\boldsymbol{\Sigma}_{p}\right\rangle$ has the 
form

$$
\left\langle\boldsymbol{\Sigma}_{p}\right\rangle=-n k T \boldsymbol{I}+n\left[\left\langle\boldsymbol{S}^{E}\right\rangle+\left\langle\boldsymbol{S}^{B}\right\rangle+\left\langle\boldsymbol{S}^{P}\right\rangle\right]
$$

where $-n k T I$ is simply the osmotic pressure due to the thermal kinetic energy of the Brownian particles and $\left\langle\boldsymbol{S}^{E}\right\rangle,\left\langle\boldsymbol{S}^{B}\right\rangle$ and $\left\langle\boldsymbol{S}^{P}\right\rangle$ are the average hydrodynamic, Brownian and interparticle-force particle stresslets (symmetric first moment of the force distribution integrated over the particle surface). The number density of particles is given by $n$, and $k$ and $T$ are the Boltzmann constant and fluid temperature, respectively. The particle stresslets include the stress due to the many body interactions among all the particles, and this is the part that we compute via particle simulations. At any given time the particle stresslets are a function only of the instantaneous particle configuration. Finally, as defined in this work the effective bulk viscosity of the suspension is given by

$$
\kappa_{e f f}=\left(\kappa+\frac{4}{3} \eta \phi\right) \frac{1}{1-\phi}+\kappa^{E}+\kappa^{B}+\kappa^{P}
$$

where the last three terms are the hydrodynamic, Brownian and interparticle-force contributions to the bulk viscosity coming from many-body interactions and are obtained directly from the corresponding average particle stresslets. The first term gives the single particle correction to the bulk viscosity in a uniformly expanding fluid.

The direct hydrodynamic contribution to the stress due to the disturbance flows caused by the finite size of the particles in an imposed flow is given by $n\left\langle\boldsymbol{S}^{E}\right\rangle$, and it is directly proportional to the applied rate of deformation. The Péclet number for an expansion flow defined as

$$
P e=\frac{\frac{1}{3}\langle e\rangle b^{2}}{2 D}
$$

is the rate of expansion $\langle e\rangle$ nondimensionalized by the diffusive time-scale of the particle $b^{2} / 2 D$. Here $D=k T / 6 \pi \eta b$ is the Stokes-Einstein-Sutherland diffusivity of an isolated particle, and each particle acts as a hard sphere of radius $b$. The corresponding hydrodynamic contribution to the bulk viscosity is obtained from the hydrodynamic stresslet as

$$
\kappa^{E}=\frac{n\left\langle S^{E}\right\rangle}{3\langle e\rangle},
$$


and therefore to first order in $P e$ it can be computed directly from the equilibrium microstructure because the scaling with the rate of deformation $\langle e\rangle$ cancels out. Here $S$ denotes the trace of the corresponding stresslet, i.e., $\langle\boldsymbol{S}\rangle=\frac{1}{3}\langle S\rangle \boldsymbol{I}$ for an isotropic stresslet. In practice the hydrodynamic contribution to the bulk viscosity for small deviations from equilibrium $(|P e| \ll 1)$ is computed by averaging the hydrodynamic stress over an ensemble of equilibrium particle configurations.

The Brownian and interparticle-force contributions to the stress have a finite average value at equilibrium independent of the rate of deformation because they originate from the thermal motion of the particles. Deviations from the equilibrium value occur only when the microstructure is perturbed from equilibrium. The corresponding contributions to the bulk viscosity are given by

$$
\kappa^{B}=\frac{n\left\langle S^{B}\right\rangle-n\left\langle S^{B}\right\rangle^{e q}}{3\langle e\rangle} \quad \text { and } \quad \kappa^{P}=\frac{n\left\langle S^{P}\right\rangle-n\left\langle S^{P}\right\rangle^{e q}}{3\langle e\rangle}
$$

where the superscript $e q$ denotes the equilibrium value. The $\kappa^{B}$ and $\kappa^{P}$ contributions can be evaluated numerically from dynamic simulation by generating deviations from equilibrium in the microstructure and computing the resulting change in the stress. However, for small deviations from equilibrium it becomes difficult to isolate the excess stress caused by the imposed flow from thermal fluctuations in the stress and increasingly longer simulations would be required to get good averaging and obtain an accurate value for the bulk viscosity contributions. We avail ourselves of an alternate approach to evaluating the transport properties at equilibrium, using Green-Kubo relations to get them from stress autocorrelation functions [Green 1952]. The stress autocorrelation function characterizes the nature and rate of relaxation of fluctuations in the stress due to Brownian motion. For the shear viscosity one would use the shear stress autocorrelation function at equilibrium, and for the bulk viscosity the pressure autocorrelation is used as [Nagèle and Bergenholtz 1998]

$$
\kappa^{B}=\frac{V}{k T} \int_{0}^{\infty}\langle\delta \Pi(0) \delta \Pi(t)\rangle \mathrm{d} t
$$

where the angle brackets denote an ensemble average, $V$ is the volume of the system over which the averaging is done, $t$ is the time, and $\delta \Pi(t)$ is the instantaneous deviation of the osmotic pressure of the suspension from its equilibrium value. Nondimensionalizing the pressure fluctuations with $n k T$ 
and time with the relative diffusive time of the particles as $\tau=t\left(2 D / b^{2}\right)$ we obtain the relation

$$
\overline{\kappa^{B}}=\frac{9}{4} N \phi \int_{0}^{\infty}\langle\overline{\delta \Pi}(0) \overline{\delta \Pi}(\tau)\rangle \mathrm{d} \tau
$$

where $N$ is the total number of particles in the volume $V, \phi$ is the volume-fraction of the particles and $\overline{\kappa^{B}}=\left(\kappa^{B}+\kappa^{P}\right) / \eta$ is the total Brownian contribution to the bulk viscosity nondimensionalized with the fluid viscosity. We define the nondimensional pressure autocorrelation function as

$$
C^{P}(\tau)=\langle\overline{\delta \Pi}(0) \overline{\delta \Pi}(\tau)\rangle
$$

In the absence of hydrodynamic interactions the direct Brownian contribution to the stress $n\left\langle\boldsymbol{S}^{B}\right\rangle$ arising from interactions between the particles as they undergo Brownian motion becomes zero and only the interparticle-force contribution $n\left\langle S^{P}\right\rangle$ remains. This work is restricted to hard spheres so that the interparticle-force stress comprises only the force due to hard sphere collisions $\boldsymbol{F}^{P}=\frac{1}{2} \boldsymbol{n} \delta(r-$ $2 b$ ), where $\boldsymbol{n}$ is the normal vector along the line joining the centers of two touching spheres and $\delta$ is the delta function at the surface of contact. The interparticle force produces the total Brownian stress in the case of hard spheres undergoing Brownian motion. Conversely, in the presence of hydrodynamic interactions the particle surfaces never touch due to the strong lubrication forces near contact and accordingly $n\left\langle S^{P}\right\rangle=0$, while $n\left\langle S^{B}\right\rangle$ accounts for all of the Brownian stress. The hard sphere nature of the particles is preserved by the no-slip hydrodynamic boundary condition on the particle surface. In the following sections we describe the determination of the bulk viscosity via Brownian Dynamics in the absence of hydrodynamics, and using Stokesian Dynamics [Brady and Bossis 1988] and Accelerated Stokesian Dynamics — near field [Banchio and Brady 2003] simulations to account for the presence of hydrodynamic interactions.

\subsection{Brownian Dynamics simulations}

The "potential-free" technique developed by Foss and Brady [2000] was employed to perform Brownian Dynamics simulations of hard spheres at equilibrium. Each particle is given a random Brownian kick at each time step, and particle collisions are resolved by moving the overlapped particles away from each other along the line joining their centers until they are touching. The 
interparticle force due to hard sphere interactions for each particle is estimated as

$$
\boldsymbol{F}^{P}=6 \pi \eta b \frac{\Delta \boldsymbol{x}^{H S}}{\Delta t}
$$

where $\Delta \boldsymbol{x}^{H S}$ is the particle displacement from resolution of all the overlaps with other particles in time step $\Delta t$. This can be thought of as the average Stokes drag on the particle during the course of the hard-sphere displacement. The particle-phase contribution to the stress due to the collisions is defined as the average stress over the volume $V$ containing the $N$ particles and is given by

$$
\left\langle\Sigma^{P}\right\rangle=-n k T \boldsymbol{I}-n\left\langle\boldsymbol{x} \boldsymbol{F}^{P}\right\rangle
$$

where the angle brackets denote an average over all the particles, $\boldsymbol{x}$ is the particle position and $n$ is the number density of particles. Since the particle collisions result from Brownian motion of the particles, the stress computed from resolving the overlaps due to collisions gives the Brownian stress of the particle phase. Note that there is a finite error associated with the interparticle force computed from 5.8 as the $O(\Delta t)$ error in $\Delta \boldsymbol{x}^{H S}$ also gets divided by $\Delta t$. Further, as the volume fraction increases the number of collisions to be resolved at each time step would also increase and therefore the accumulated error in the stress calculation is expected to increase with increasing $\phi$.

All the runs were started with particle configurations generated using a modified LubachevskyStillinger algorithm, in which hard spheres are grown in size and evolved according to Newtonian dynamics with periodic boundary conditions along the cell sides [Skoge et al. 2006]. The codes implementing this algorithm are generously available from Professor Torquato and were used for generating the starting configurations. Simulations were performed with $N=100$ and 1000 particles, with periodic boundary conditions applied at the simulation cell boundaries to emulate an infinite suspension. A time step of $\Delta \tau=2 \times 10^{-4}$ in units of the diffusive time-scale was used for a total time of $\tau=4000$ for all volume fractions, broken up into eight distinct runs of $\tau=500$ for each $\phi \leq 0.4$ and sixteen runs each of $\tau=270$ for $\phi>0.4$. The initial time steps in each simulation up-to $\tau=20$ were not included in calculation of the autocorrelation functions as the starting configuration was allowed to equilibrate during that time. The particles were allowed to undergo Brownian motion without any affine displacement and all the components of the stress tensor arising from 
collisions between particles were recorded at each time step. The instantaneous particle pressure was calculated as the trace of the stress tensor averaged over all particles:

$$
\Pi(t)=\frac{1}{N} \sum_{i=1}^{N}\left(\Sigma_{x x}^{P}+\Sigma_{y y}^{P}+\Sigma_{z z}^{P}\right)_{i} / 3
$$

and was averaged over the entire duration of the simulation to obtain the equilibrium pressure for a given $\phi$. Simulations were performed over a range of volume-fractions from $\phi=0.05$ to 0.55 .

\subsubsection{Simulation results}

The equilibrium pressure obtained from the simulations is shown in Figure 5.1 and serves as a primary check for the validity of the simulation method. The analytical value of the equilibrium osmotic pressure given by

$$
\bar{\Pi}_{0}=1+4 \phi g_{0}(2 ; \phi)
$$

is also shown, where $g_{0}(2 ; \phi)$ is the equilibrium pair-distribution function at contact evaluated using the well known Carnahan-Starling (CS) equation of state [Carnahan and Starling 1969] for $\phi \leq 0.55$ :

$$
g_{0}(2 ; \phi)=\frac{1-\frac{1}{2} \phi}{(1-\phi)^{3}}
$$

For higher volume fractions we have used the very precise values for $g_{0}(2 ; \phi)$ determined by Rintoul and Torquato [1996], which also match the CS equation of state at its limiting value of $\phi=0.5$. The osmotic pressure obtained from Brownian Dynamics is seen to slightly underestimate the theoretical value. We attribute this discrepancy to the error associated with the determination of the interparticle force from particle collisions, which is larger for higher volume fractions as the number of collisions to be resolved increases.

The pressure autocorrelation function was evaluated as an ensemble average over all the particle configurations over the course of the simulation. Specifically, for any given simulation run

$$
\langle\overline{\delta \Pi}(0) \overline{\delta \Pi}(\tau)\rangle=\frac{1}{M-\tau / \Delta \tau} \sum_{m=0}^{M-\tau / \Delta \tau} \overline{\delta \Pi}(m \Delta \tau) \overline{\delta \Pi}(m \Delta \tau+\tau)
$$

where $M$ is the total number of time steps from the run included in the calculation, and $\overline{\delta \Pi}(t)=$ 
$\bar{\Pi}(t)-\langle\bar{\Pi}\rangle$. Further averaging was done over all the runs performed for a given $\phi$. Since the pressure was calculated as an average of the diagonal components of the stress, the cross-correlations among the diagonal stress components are automatically included in the above expression for the pressure autocorrelation function.

Figure 5.2 and 5.3 show the pressure autocorrelation function from Brownian Dynamics simulations for various $\phi$ with $N=100$ and 1000 particles, respectively. In the short-time limit the data exhibits the expected $t^{-1 / 2}$ scaling [Cichocki and Felderhof 1991; Brady 1993b]. At longer times the decay is very slow due to the isotropic nature of the dissipation of pressure fluctuations. Figure 5.4 shows the simulation data scaled to match the theoretical prediction for the pressure autocorrelation function for $\phi=0.2$ to 0.35 . The analytical expression for the scaled pressure autocorrelation function using the time-scale for simulations $\tau=t\left(2 D / b^{2}\right)$ is given by

$$
\overline{C_{N H}^{P}}(\tau)=\frac{4}{3}\left(\frac{2}{\sqrt{\pi \tau}}-e^{\tau / 4} \operatorname{Erfc}\left(\sqrt{\frac{\tau}{4}}\right)\right)
$$

where $\overline{C_{N H}^{P}}(\tau)=C_{N H}^{P}(\tau) N /\left(\phi g_{0}(2 ; \phi)\right)$ as described in the next section. At short times the pressure autocorrelation function is predicted to scale as $t^{-1 / 2}$ with the asymptote $\overline{C_{N H}^{P}} \sim 4 / 3(2 / \sqrt{\pi \tau}-1)$, and as $t \rightarrow \infty$ it decays as $\overline{C_{N H}^{P}} \sim(16 / 3 \sqrt{\pi}) \tau^{-3 / 2}$. The pressure autocorrelation data is in excellent agreement with the theoretical prediction in the $t \rightarrow 0$ limit. At longer times the data for the smallest volume fraction $\phi=0.05$ is in good agreement with the theory but there are variations in the rate of decay at higher volume fractions. Both the $N=100$ and $N=1000$ systems exhibit similar behavior so the system size apparently does not have any effect on the pressure autocorrelation data when periodic boundary conditions are imposed. For 1000 particles at $\phi>0.52$ the particles were prone to crystallization resulting in a pressure autocorrelation function that is much higher and does not decay at all. The crystallization took place starting at $\phi>0.5$ with 100 particles.

For all the data the $t^{-3 / 2}$ rate of decay of the pressure autocorrelation function is so slow that the data gets reduced to just noise before being able to capture the long-time behavior for both the $N=100$ and $N=1000$ systems. Therefore it is important to know from theory how the longtime tails decay so that the pressure autocorrelation function can be integrated correctly to find the bulk viscosity. The long-time tails were obtained by fitting the analytical curve from dilute theory to the region shortly before the data becomes too noisy. The noise in the simulation results could 
be reduced by using a smaller time-step thereby capturing more of the long-time behavior, but that would also increase the computational cost significantly. Having analytical knowledge of the pressure autocorrelation curve alleviates the need for more simulation data for the long-time tails, particularly because it is apparent from the figures that the simulation results follow the analytical curve in the regions of low noise. We simply fit the dilute theory curve to the simulation data in the region before the data becomes too noisy in order to obtain the long-time tails.

The Green-Kubo bulk viscosity in the absence of hydrodynamic interactions is shown in Figure 5.9. The Molecular Dynamics simulation results of Sigurgeirsson and Heyes [2003] for the bulk viscosity of hard sphere fluids scaled with the zero-density bulk viscosity are also shown for comparison. Both the data are in reasonable agreement at small $\phi$ and have the same scaling at large $\phi$ but there are significant differences in the intermediate $\phi$ regime. Although both the hard sphere systems must have the same equilibrium properties, the mechanism of stress dissipation is different in $\mathrm{MD}$ and $\mathrm{BD}$ simulations, hence the transport properties need not be identical. The particle motion in BD is heavily damped because of the drag force exerted by the surrounding fluid, therefore particle momentum is not conserved. Additionally, at each time-step the particles experience a random Brownian kick which completely changes the spatial distribution of particle momentum. On the other hand, in MD simulations the particles start with an initial set of positions and momenta which may be random but as the simulation proceeds the particle collisions are such that the total momentum is conserved. Thus the distribution of momentum in time and space is different in BD and MD. Since the dynamic properties such as the shear and bulk viscosity depend on the rate of change of momentum in addition to the spatial distribution of particles, we do not expect the transport properties obtained from $\mathrm{BD}$ and $\mathrm{MD}$ to be identical.

The shear stress autocorrelation function was also determined from the $N=1000$ particle systems for validation of the simulations, and is shown in Figure 5.10. It decays much faster as $t^{-7 / 2}$ and consequently the long-time tail is captured correctly in the simulation results. The Brownian shear viscosity $\eta_{0}^{P}$ was calculated by numerically integrating the shear stress autocorrelation function without any fitting because the noise in the data is adequately low. The Green-Kubo expression for shear viscosity in nondimensional form given by [Nagèle and Bergenholtz 1998]

$$
\overline{\eta^{B}}=\frac{9}{4} N \phi \int_{0}^{\infty}\left\langle\overline{\Sigma_{x y}}(0) \overline{\Sigma_{x y}}(\tau)\right\rangle \mathrm{d} \tau
$$


was used to calculate $\overline{\eta^{B}}$, which is the Brownian contribution to the shear viscosity scaled with the fluid viscosity. Since there is no preferred direction at equilibrium the $y z$ and $x z$ components of the stress were also autocorrelated and included in the ensemble average in the Green-Kubo formula. Figure 5.12 shows the Green-Kubo shear viscosity in the absence of hydrodynamic interactions scaled with the fluid viscosity along with the simulation results of Foss and Brady [2000], which are noticeably lower than our results at higher volume fractions. The difference is due to the higher accuracy of the results from the present work because the time-step used is smaller (Foss and Brady used a time-step of $\Delta \tau=2.5 \times 10^{-4}$ ), and also because we fitted the analytical dilute theory curve to the simulation data in order to obtain the long-time tails while Foss and Brady simply fitted the tails with $t^{-7 / 2}$, thereby losing some area under the stress autocorrelation curve. The total shear viscosity for Brownian particles in a suspension would also includes the high frequency dynamic

viscosity, given by $\eta_{\infty}^{\prime}=\eta\left(1+\frac{5}{2} \phi\right)$ in the absence of inter-particle hydrodynamic interactions. The high frequency dynamic viscosity represents the direct viscous contribution to the suspension stress.

\subsubsection{Scaling with volume fraction}

Next we turn our attention to the scaling of the stress autocorrelation functions with $\phi$. The Smoluchowski description of the particle microstructure was used to relate the suspension stress to the pair-distribution function [Brady 1993a]. In the absence of hydrodynamics the entire interparticle contribution to the stress comes only from collisions between touching particles, so the stress can be expressed as an integral of the pair-distribution function at contact:

$$
n\left\langle\boldsymbol{S}^{P}\right\rangle=-n^{2} k T b \int_{r=2 b} \hat{\boldsymbol{r}} \hat{r} g(\boldsymbol{r}) d S,
$$

where $\hat{\boldsymbol{r}}=\boldsymbol{r} / r$ is the outward normal on the reference particle surface. Small departures from equilibrium in the microstructure can be expressed as a regular perturbation in $P e$ about the equilibrium microstructure as

$$
g(\boldsymbol{r}, \tau)=g_{0}(\boldsymbol{r})[1+\operatorname{Pef}(\boldsymbol{r}, \tau)]
$$

where $f(\boldsymbol{r}, \tau)$ is the first-order perturbation about equilibrium independent of $P e$. Here the Péclet number is defined based on the bare diffusivity of a particle, which determines the time-scale for 
decay of Brownian fluctuations. However as the volume fraction increases the rate of decay of fluctuations slows down as is evident from Figure 5.5 and Figure 5.11. Therefore the characteristic time scale of relaxation of the microstructure must be based on the volume fraction dependent characteristic diffusivity defined as $D \hat{D}(\phi)$, where $D$ is the bare diffusivity and $\hat{D}(\phi)$ provides the scaling with $\phi$. Hence we define

$$
\begin{aligned}
\hat{P} e & =P e / \hat{D}(\phi) \\
\hat{f}(\boldsymbol{r}) & =\hat{D}(\phi) f(\boldsymbol{r}) \\
\hat{\tau} & =\hat{D}(\phi) \tau
\end{aligned}
$$

as the scaled Péclet number, scaled perturbation about equilibrium and the scaled time, respectively. The appropriate relaxation time scale for the microstructure is now given by $b^{2} / \hat{D}(\phi) D$. With these scaled variables we can scale up the dilute-theory results for $\hat{f}(\boldsymbol{r})$ and get a reasonable estimate for $f(\boldsymbol{r})$ for all volume fractions. The perturbation in an expansion flow is isotropic and therefore $\hat{f}$ is a function only of the radial distance $r$ between two particles. Consequently, the stress disturbance is also isotropic and is only present in the trace of the bulk stress. Substituting the expression for $g(\boldsymbol{r})$ into (5.16) gives the following expression for the disturbance in the stress:

$$
n \frac{1}{3}\left\langle S^{P}\right\rangle \boldsymbol{I}+\Pi_{0} \boldsymbol{I}=-6 \phi^{2} \eta\langle e\rangle \frac{g_{0}(2 ; \phi)}{\hat{D}(\phi)} \hat{f}(2 b) \boldsymbol{I}
$$

where $n\left\langle S^{P}\right\rangle$ is the trace of the particle phase stress. The solution for $\hat{f}(2 b)$ with the scaling in (5.30a) is obtained from the Smoluchowski equation as $\hat{f}(2 b)=-2$ (note the factor of 2 in the diffusive time scale used for the dilute theory). The bulk viscosity can now be derived for all volume fractions using equation (5.5) as

$$
\frac{\kappa^{B}}{\eta}=12 \phi^{2} \frac{g_{0}(2 ; \phi)}{\hat{D}(\phi)}
$$

The bulk viscosity can also be calculated from (5.7), therefore equating the two expressions for $\kappa^{B}$ we get

$$
12 \phi^{2} \frac{g_{0}(2 ; \phi)}{\hat{D}(\phi)}=\frac{9}{2} \frac{N \phi}{\hat{D}(\phi)} \int_{0}^{\infty}\langle\overline{\delta \Pi}(0) \overline{\delta \Pi}(\hat{\tau})\rangle \mathrm{d} \hat{\tau}
$$


where the pressure autocorrelation function is now a function of the diffusive time scaled with $\hat{D}(\phi)$. The temporal scaling cancels out as it does not affect the magnitude of the pressure fluctuations, rather it only prescribes the rate of decay of fluctuations.

Equation (5.21) suggests that defining the scaled pressure autocorrelation function as

$$
\overline{C_{N H}^{P}}=\frac{N}{\phi g_{0}(2 ; \phi)} C_{N H}^{P}
$$

should collapse the data for all volume fractions and for all values of $N$ onto the predicted theoretical curve. Intuitively, in the absence of hydrodynamic interactions particles only interact with their immediate neighbors. Hence the only change that any given particle experiences as the volume fraction is changed is the pair-distribution function at contact and the change in the particle density around it. This simple argument too would suggest scaling the magnitude of the pressure autocorrelation function with $\phi g_{0}(2 ; \phi)$. Indeed, the scaling in (5.21) collapses all the simulation data close to the analytical curve for the pressure autocorrelation function (5.14), as shown in Figure 5.4 and 5.5 .

\subsubsection{Temporal scaling}

The collapse of the scaled pressure autocorrelation data onto the analytical curve is perfect in the short-time limit but at longer times the rate of decay deviates from the analytical curve by varying amounts depending on the volume fraction. The pressure autocorrelation obtained from Brownian Dynamics simulations for volume fractions up-to $\phi=0.35$ are shown in Figure 5.4 for $N=1000$ and $N=100$. The agreement of the simulation data with the analytical curve is very good for $\phi=0.05$, but as $\phi$ increases there is a perceptibly faster decay of the pressure autocorrelation up to $\phi=0.2$, where it stops becoming faster. For $\phi \geq 0.4$ the rate of decay is slower with increasing $\phi$ as shown in Figure 5.5, indicating an increase in the time scale for relaxation.

For additional guidance we also look at the shear stress autocorrelation $C_{N H}^{S}=\left\langle\overline{\Sigma_{x y}}(0) \overline{\Sigma_{x y}}(\tau)\right\rangle$, shown in Figure 5.10. The simulation data for $C_{N H}^{S}$ has much lesser noise and captures the long-time decay correctly. Since both the shear and the pressure autocorrelation are just different measurements from the same physical process we expect their magnitude and temporal behavior to have 
similar scaling with $\phi$. The scaled shear stress autocorrelation defined as

$$
\overline{C_{N H}^{S}}=\frac{N}{\phi g_{0}(2 ; \phi)} C_{N H}^{S},
$$

is shown in Figure 5.11(a) and indeed for small volume fractions it coincides very well with the dilute-theory analytical curve 5.14. There is a slight decrease in the rate of decay with increasing $\phi$ at small values of $\phi$ but it is very slight compared to the variations in the pressure autocorrelation function. The theoretical curve for $\overline{C_{N H}^{S}}$ was obtained from a numerical inverse Laplace transform [Hollenbeck 1998] of the frequency-dependent shear viscosity for hard spheres without hydrodynamics. It is quite clear from both figures that the time scale of relaxation is constant for $\phi \leq 0.35$ and increases monotonically with $\phi$ for higher volume fractions, similar to $\overline{C_{N H}^{P}}$ in Figure 5.8(a). Therefore the characteristic diffusivity scaling for stress relaxation must be $\hat{D}(\phi)=1$ for $\phi \leq 0.35$ and a decreasing function of $\phi$ for $\phi>0.35$.

The exact behavior of the characteristic diffusivity $\hat{D}(\phi)$ governing the relaxation of fluctuations is not clear from theory, and several quantities have been suggested earlier. Brady [1993b] suggested scaling time with the equilibrium short-time self-diffusivity as $\hat{D}(\phi)=D_{0}^{s}(\phi) / D$, as it accounts for the slowing down of the dynamics due to stronger hydrodynamic interactions between particles as the volume fraction increases. He successfully used this scaling to collapse the experimental data of van der Werff et al. [1989] for the frequency-dependent dynamic viscosity for the concentration range $0.46 \leq \phi \leq 0.6$. In the absence of hydrodynamic interactions the short-time self-diffusivity is simply the bare diffusivity for all $\phi$ and therefore scaling with $D_{0}^{s}(\phi)$ would not account for the variations in temporal decay in our simulations.

A more suitable choice for the temporal scaling is the equilibrium long-time self-diffusivity $D_{\infty}^{s}(\phi)$ because it incorporates the effects of particle interactions with and without hydrodynamics. The long-time self diffusivity corresponds to the motion of a particle on times long compared to $a^{2} / D$ so that the particle has wandered far compared to its size, and in doing so exchanged places with its neighbors and experienced many different configurations [Brady 1994]. The time-scale of stress relaxation would scale as $D_{\infty}^{s}$ if it was necessary for the particles to exchange places with a neighbor in order to achieve a significantly different particle configuration such that the fluctuations in particle-phase stress are no longer correlated. This is the case at high volume fractions when par- 
ticles are likely to get trapped in a 'cage' of neighboring particles. At low volume fractions particles have more freedom to move around so that the particles can achieve significantly different configurations via collisions with neighboring particles alone, without having to travel far or exchange places with a neighbor. This explains the uniform rate of stress relaxation at low concentrations and the slowing down of the stress relaxation at higher concentrations. At intermediate concentrations the particles would only need to distort the surrounding cage sufficiently without actually breaking out of it for the microstructure to relax to a different state. Hence the relaxation time-scale would be influenced by $D_{\infty}^{s}$ but not be completely determined by it.

Our simulations indicate that the temporal scaling with $D_{\infty}^{s}$ should start at approximately $\phi=$ 0.35 . For smaller volume fractions the temporal scaling can be quantified by computing the relaxation time directly from the stress autocorrelation functions. From Figure 5.4 it is evident that the change in relaxation rate does not depend on the number of particles, therefore it must be the result of some physical process. The relaxation time for the pressure autocorrelation is defined as

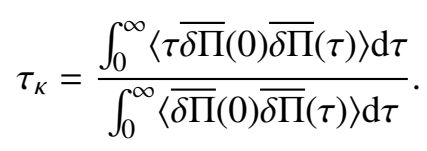

However, since computing the relaxation time over the entire range of $\tau$ would require fitting the tails to a known curve (for which we don't know the correct temporal scaling yet) we compute

$$
\tau_{\kappa}(0.7)=\frac{\int_{0}^{0.7}\langle\tau \overline{\delta \Pi}(0) \overline{\delta \Pi}(\tau)\rangle \mathrm{d} \tau}{\int_{0}^{0.7}\langle\overline{\delta \Pi}(0) \overline{\delta \Pi}(\tau)\rangle \mathrm{d} \tau}
$$

instead, i.e., the integral is evaluated only up-to $\tau=0.7$ where the data has not yet become too noisy. Besides, the time scale integral computed with $\tau \rightarrow \infty$ in (5.25) will be unbounded because $\langle\tau \overline{\delta \Pi}(0) \overline{\delta \Pi}(\tau)\rangle \sim t^{-1 / 2}$ as $\tau \rightarrow \infty$. The change in the relaxation rate is apparent even within this short time boundary, hence $\tau_{\kappa}(0.7)$ should give a reasonable estimate for the change in temporal scaling. The $\tau_{\kappa}(0.7)$ data is shown in Figure 5.6. There is a rapid decrease in the relaxation time-scale for $\phi \leq 0.2$ and after that the relaxation rate is almost constant, indicating that there are possibly two competing effects governing the time scale. At very small volume fractions, the addition of more particles in the system would cause an increase in the number of collisions taking place and thereby help in dissipating the stress and microstructural deformation faster. At small $\phi$, an increase in 
the particle density is not sufficient to hinder the motion of the particles. Therefore there is a net decrease in the time scale of relaxation as more collisions are taking place but the hindrance due to the additional collisions is not enough to perceptibly slow down the movement of the particles. As $\phi$ increases the hindering effect of more particles eventually catches up and balances out the entropic effect that was helping to dissipate the stress, so that the relaxation time-scale stops changing after $\phi=0.2$. Since there is no clear scaling to account for the competing effects we simply use the polynomial fit given by $\tau_{f i t}(\phi)$ for the temporal scaling in this regime. For higher volume fractions the relaxation time-scale starts increasing due to 'caging' effects and therefore $D_{\infty}^{s}$ is a better scaling for $\phi \geq 0.35$.

Accordingly we define the characteristic diffusivity scaling as

$$
\hat{D}_{N H}(\phi)= \begin{cases}\tau_{f i t}(0.05) / \tau_{f i t}(\phi), & \text { if } \phi<0.35 \\ \frac{D_{\infty, N H}^{s}(\phi) \tau_{f i t}(0.05)}{D_{\infty, N H}^{s}(0.35) \tau_{f i t}(0.35)}, & \text { if } \phi \geq 0.35\end{cases}
$$

so that $\hat{D}_{N H}(\phi)$ is a continuous function of $\phi$, where the subscript $N H$ stand for no hydrodynamics. Here $D_{\infty}^{s}(\phi)$ is the long-time self-diffusivity in the absence of hydrodynamic interactions. The values for $D_{\infty}^{s}$ were calculated from interpolation of the simulation results of Foss [1999]. The pressure autocorrelation data plotted against time scaled as $\hat{\tau}=t b^{2} / D \hat{D}_{N H}(\phi)$ is shown in Figure 5.8(b), and the shear stress autocorrelation data with the same temporal scaling is shown in Figure 5.11(b). For both sets of data the long-time tails were obtained by fitting the corresponding analytical curves to the simulation data with the temporal scaling given by (5.26). The simulation results and the fitted tails collapse quite nicely onto the theoretical curve with this scaling. The time-scale of decay for larger times including the long time tails given by $\tau_{\kappa}(200)$ and shown in Figure 5.7 has roughly the same behavior as $\tau_{\kappa}(1)$. The scaled theoretical prediction for the bulk viscosity given by (5.20) is shown in Figure 5.9 along with the Brownian Dynamics simulation results. The MD simulation results of Sigurgeirsson and Heyes [2003] for the bulk viscosity of hard spheres scaled with the zero-density shear viscosity are also shown on the same plot. The MD data does not have the same scaling as our BD data for $\phi<0.35$ because the entropic effect of more Brownian particles helping to dissipate the stress with increasing $\phi$ is absent. For higher $\phi$ the MD data also scales as $D_{\infty}^{s}$ as 
expected. The theoretical prediction is in good agreement with our BD results with $N=1000$ and $N=100$ particles. The scaled theory for the shear viscosity is shown in Figure 5.12 and is in very good agreement with the BD results.

\subsection{Effect of hydrodynamic interactions}

The primary effect of hydrodynamic interactions is to dampen particle collisions due to the lubrication flows between nearly touching particle surfaces, resulting in stress autocorrelations that plateau linearly to a constant value in the $t \rightarrow 0$ limit. The hard sphere nature of the particles is preserved by the no-slip hydrodynamic boundary condition at contact. Equilibrium simulations of particles undergoing Brownian motion were performed using full Stokesian Dynamics (SD) with $N=27$ particles and the Accelerated Stokesian Dynamics — near field (ASDB-nf) method with $N=1000$ particles. In the SD method, the full far-field and near-field hydrodynamic interactions arising from Brownian motion are computed between all particles at each time step. Since the Brownian forces are uncorrelated in time the velocities from previous time steps cannot be used and fresh calculations must be performed at every step. This requirement makes the SD technique very slow because the computational time of the calculations is $O\left(N^{3}\right)$, therefore only small particle systems with periodic boundaries were simulated with this method. Simulations were performed for a total time of $\tau_{f}=500$ with a time step of $\Delta \tau=5 \times 10^{-4}$ for $\phi \leq 0.4$ and $\Delta \tau=10^{-4}$ for $\phi>0.4$, where $\tau=t b^{2} / D$. Although the lubrication forces should prevent particles from touching, some overlap does occur at high concentrations due to the finite time step implemented in simulations. The particle overlaps are not explicitly resolved in SD and the particles normally move away without the overlap becoming too severe.

The ASDB-nf method of Banchio and Brady [2003] is a much faster $O(N)$ procedure where the Brownian forces arising from far-field interactions are approximated in a mean-field manner and only the near-field lubrication interactions are actually evaluated between particles. A time step of $\Delta \tau=10^{-3}$ was used for all the ASDB-nf simulations with a total time of $\tau_{f}=1500$. Particle overlaps were explicitly resolved at each time step by moving the overlapped particles away from each other along the line joining their centers until they are touching. The far-field diffusivities used for the mean-field approximation are already corrected for an infinite system, hence the hydrody- 
namic interactions evaluated using this technique are independent of the number of particles in the simulation cell. Finite-size effects may appear only due to the periodic boundary conditions used for evaluating particle collisions. The far-field contribution to the pressure fluctuations is negligible because at equilibrium the osmotic pressure is completely determined by the lubrication forces at contact as shown by Brady [1993a]. Therefore the particle pressure in the ASDB-nf method is computed solely from pairwise lubrication interactions with a cutoff radius of $r=4 b$, so that the favorable $O(N)$ computational scaling is preserved. The SD simulations explicitly account for all the far-field and near-field interactions for the pressure calculation and we shall see that there is no noticeable difference between the SD and ASDB-nf results apart from finite-size effects due the different number of particles simulated.

\subsubsection{Scaling with volume fraction}

It is instructive to look at how the $\phi$ scaling of the pressure autocorrelation function changes in the presence of hydrodynamic interactions before analyzing the simulation results. From dilute theory the perturbation to the equilibrium microstructure in the steady expansion linear-response limit is given by the quadrature

$$
f(r)=-\int_{r}^{\infty} \frac{1}{G(r)}\left[\frac{8}{r^{2}}+v^{\prime}(r)\right] \mathrm{d} r
$$

where $G(r)=x_{22}^{a}-x_{21}^{a}$ [Kim and Karrila 1991] is the scalar hydrodynamic function for the radial component of the relative mobility of two particles, $\boldsymbol{D}=2 D[G(r) \hat{\boldsymbol{r}} \hat{\boldsymbol{r}}+H(r)(\boldsymbol{I}-\hat{\boldsymbol{r}} \hat{\boldsymbol{r}})]$ [Batchelor 1976]. The inter-particle disturbance velocity $v^{\prime}(r)$ can also be expressed in terms of the hydrodynamic functions as

$$
v^{\prime}(r)=\frac{4}{3} G(r)\left(X_{22}^{P}(r)-X_{21}^{P}(r)\right)
$$

where $X_{\alpha \beta}^{P}(r)$ are the hydrodynamic resistance functions relating the disturbance force on a particle to the imposed expansion flow, determined by Jeffrey et al. [1993].

Departures from equilibrium in a non-dilute suspension are resisted by the effective relative diffusivity between two particles in the suspension resulting from hydrodynamic interactions with all the particles. As the particles become well separated $\boldsymbol{D}$ asymptotes to $D D_{0}^{s}(\phi) \boldsymbol{I}$, the short-time selfdiffusivity of a particle and not the infinite dilution value $D$. Brady [1993b] used this argument to rescale time and the Péclet number with $D_{0}^{S}(\phi)$, thereby proposing that the appropriate time scale 
for viscoelastic response is $b^{2} / D D_{0}^{s}(\phi)$. However, as discussed in the previous section this scaling does not explain the rate of stress relaxation observed in our simulations. Instead we propose that the short-time self-diffusivity primarily serves as the scaling for the strength of hydrodynamic interactions between particles, i.e., we multiply the hydrodynamic mobility function $G(r)$ with $D_{0}^{s}(\phi)$ and the resistance functions $X_{\alpha \beta}^{P}(r)$ with $1 / D_{0}^{s}(\phi)$ to extend the dilute theory for all concentrations. Thus the perturbation for all $\phi$ can be approximated as

$$
f(r)=-\frac{1}{D_{0}^{s}(\phi)} \int_{r}^{\infty} \frac{1}{G(r)}\left[\frac{8}{r^{2}}+v^{\prime}(r)\right] \mathrm{d} r
$$

where $D_{0}^{s}(\phi)$ is nondimensionalized with the bare diffusivity $D$ and contains all the $\phi$ dependence of the hydrodynamics. The scaling of $v^{\prime}(r)$ does not change because the inverse $\phi$ dependence in the mobility and resistance functions cancels out.

The temporal scaling for stress relaxation $\hat{D}_{H}(\phi)$ is not clear from theory and we will deduce it from the simulation results. Taking hydrodynamic interactions into account we define

$$
\begin{aligned}
\hat{P e} & =P e / \hat{D}_{H}(\phi) \\
\hat{f}(r) & =\hat{D}_{H}(\phi) f(r) \\
\hat{\tau} & =\hat{D}_{H}(\phi) \tau
\end{aligned}
$$

as before with the appropriate relaxation time scale for the microstructure given by $b^{2} / \hat{D}_{H}(\phi) D$. With these scaled variables $\hat{f}(r)$ satisfies the usual two-particle Smoluchowski equation with hydrodynamics for all $\phi$ with the mobility functions scaled with $D_{0}^{s}(\phi)$. The disturbance in the Brownian stress due to hydrodynamic interactions can be obtained by averaging the two-particle stress over the perturbed microstructure as

$$
n \frac{1}{3}\left\langle S^{B}\right\rangle \boldsymbol{I}+\Pi_{0} \boldsymbol{I}=-\frac{9}{2} \phi^{2} \eta \frac{\langle e\rangle}{\hat{D}_{H}(\phi)} \int_{2}^{\infty} \boldsymbol{\nabla} \cdot\left(\boldsymbol{R}_{S U} \cdot \boldsymbol{R}_{F U}^{-1}\right) g_{0}(r) \hat{f}(r) r^{2} \mathrm{~d} r
$$

where the hydrodynamic resistance tensors $\boldsymbol{R}_{S U}$ and $\boldsymbol{R}_{F U}$ are evaluated between particle pairs and $\hat{f}(r)$ is now the solution to the Smoluchowski equation with full hydrodynamics. We make a further simplification by replacing $g_{0}(r)$ with $g_{0}(2 ; \phi)$ and evaluating the integral to get the scaled dilute 
theory result for the bulk viscosity:

$$
\frac{\kappa^{B}}{\eta}=5.348 \phi^{2} \frac{g_{0}(2 ; \phi)}{\hat{D}_{H}(\phi) D_{0}^{s}(\phi)} .
$$

Comparing the expression for $\kappa^{B}$ to (5.7) suggests that the scaled pressure autocorrelation function with hydrodynamic interactions should be defined as

$$
\overline{C_{H}^{P}}=\frac{N D_{0}^{S}(\phi)}{\phi g_{0}(2 ; \phi)} C_{H}^{P}
$$

where the subscript $H$ stands for hydrodynamics. We can also estimate the short-time limit of the pressure autocorrelation function from the theoretical value of the frequency dependent bulk modulus $K^{\prime}(\omega)=\omega \kappa^{\prime \prime}(\omega)$, where $\omega$ is the frequency of oscillatory compression/expansion and $\kappa^{\prime \prime}(\omega)$ is the imaginary component of the frequency dependent bulk viscosity. Nondimensionalizing the frequency with the temporal time scale as $\hat{\alpha}=\omega b^{2} / \hat{D}_{H}(\phi) D$ and using the scaling from (5.32) we obtain the scaled dilute theory value of the high-frequency bulk modulus as

$$
K_{\infty}^{\prime}\left(\frac{b^{3}}{k T}\right)=0.567 \phi^{2} \frac{g_{0}(2 ; \phi)}{D_{0}^{S}(\phi)}
$$

The high-frequency bulk modulus is related to the zero-time limit of the pressure autocorrelation by $K_{\infty}^{\prime}=V / k T\langle\delta \Pi(0) \delta \Pi(0)\rangle$, from which we get the nondimensional zero-time limit

$$
\overline{C_{H}^{P}}(0)=2.377
$$

The $1 / D_{0}^{S}(\phi)$ scaling of the zero-time stress autocorrelation actually derives from the generalized fluctuation-dissipation theorem which gives the autocorrelation of the Brownian forces as

$$
\left\langle\boldsymbol{F}^{B}(0) \boldsymbol{F}^{B}(t)\right\rangle=2 k T \boldsymbol{R}_{F U} \delta(t)
$$

where $\boldsymbol{R}_{F U}$ is the resistance tensor giving the forces/torques on the particles due to their motion relative to the fluid. The short-time self-diffusivity $D_{0}^{s}$, which measures the average instantaneous 
mobility of a particle can be computed from an average over all configurations as

$$
D_{0}^{s}=k T\left\langle\boldsymbol{R}_{F U, i i}^{-1}\right\rangle
$$

where the subscript $i$ indicates that only the diagonal terms are included, and for the translational diffusivity only the terms giving the translational velocity are included in the average. From (5.36) and (5.37) we can deduce that $\left\langle\boldsymbol{F}^{B}(0) \boldsymbol{F}^{B}(t)\right\rangle \sim 1 / D_{0}^{s}$. Since the Brownian particle stress is simply the first moment of the force distribution it must have the same scaling with $\phi$ as the Brownian forces, thus giving $1 / D_{0}^{s}$ scaling for the Brownian stress autocorrelation. While the forces are correlated only instantaneously, the stress depends on the configuration of particles as well, hence the stress autocorrelation decays with time as the configuration changes. Equivalently, one could invoke the generalized Stokes-Einstein relation

$$
\eta_{\infty}^{\prime}(\phi) \cong \frac{1}{D_{0}^{s}(\phi)}
$$

for all volume fractions coupled with the observation that the resistance matrices must scale as $\eta_{\infty}^{\prime}$ which is the effective solvent viscosity at high volume fractions, to get the above scaling for the Brownian forces.

\subsubsection{Temporal scaling}

The average pressure computed from SD and ASDB-nf equilibrium simulations is shown in Figure 5.1 and it matches almost perfectly with the theoretical curve. This demonstrates that the approximations made in the ASDB-nf method are valid for the purpose of this analysis. The nondimensional pressure autocorrelation function $C_{H}^{P}(\tau)$ computed from SD and ASDB-nf simulations is shown in Figure 5.13 and Figure 5.14, respectively. The data from both sets of simulations has the same behavior, with a linear plateau to a constant value in the short-time limit and slowly decaying to the expected $t^{-3 / 2}$ behavior at long-times. The data degenerates to random noise before reaching the asymptotic long-time behavior. The rate of temporal decay can be seen to become slightly faster as $\phi$ increases up-to $\phi \sim 0.35$, and starts slowing down for $\phi>0.35$ similar to the trend observed in our Brownian Dynamics simulations. 
The SD simulations exhibit a sudden break in the increase of the relaxation time scale for $\phi>$ 0.45, not seen in the ASDB-nf simulations. This can be attributed to the small size of the particle system used for SD simulations $(N=27)$. The simulation cell size keeps decreasing as $\phi$ increases, and for $\phi \geq 0.45$ it is the cell length that sets the time scale of diffusion and stress relaxation. Further, the long-time decay for $\phi \geq 0.45$ seems to be almost exponential as is characteristic of diffusion in a closed box, instead of the expected asymptote to $t^{-3 / 2}$. If the particles were diffusing in a finite-sized box instead of an infinite suspension, the fluctuations in particle density would dissipate exponentially due to the finite boundary conditions. Therefore the stress autocorrelation functions would also have an exponential decay in the long-time limit. In an infinite suspension there are no spatial boundary conditions forcing an exponential decay so the decay is algebraic. However, we implement periodic boundary conditions to simulate an infinite suspension. When the simulation cell size is smaller than the length over which the particles need to diffuse for fluctuations to decay algebraically, the periodic boundary conditions begin to simulate the effect of being in a finite box thus causing an exponential decay of the stress autocorrelation functions. We will use only the ASDB-nf simulations for all the scaling analysis in order to minimize any finite-size effects. Even with the ASDB-nf simulations for $\phi>0.52, C_{H}^{P}$ decays much more slowly because the particles are in the glassy regime so that the microstructure is unable to relax by diffusion. At long times the finite size of the simulation cell causes the pressure autocorrelation to decay exponentially as the periodic boundary conditions simulate diffusion of the particles in a finite box.

Figure 5.15 shows the scaled pressure autocorrelation function $\overline{C_{H}^{P}}=C_{H}^{P} N D_{0}^{s}(\phi) /\left(\phi g_{0}(2 ; \phi)\right)$ as a function of the diffusive time $\tau$ for (a) $\phi \leq 0.35$ and (b) $\phi \geq 0.35$. The values of $D_{0}^{s}$ were taken from the ASD simulations of Sierou and Brady [2001]. The scaling works very well for collapsing all the curves in the short time limit but variations in the rate of decay at longer times prevent a complete collapse of the data over all time. The zero-time limit from scaled dilute theory given by (5.35), also shown on the plots, underestimates the simulation data by a small amount. The analytical curve for the pressure autocorrelation function without hydrodynamics is also shown, and it is clear that at long times the simulation data has the same asymptotic behavior as the analytical curve. The slight decrease in the time scale of relaxation for smaller volume fractions was not expected and needs further examination. First we need to eliminate cell-size effects so we compare the data for two different volume fractions but with $N$ such that the simulation cell has the same size for both cases. 
Figure 5.16(a) shows the data for $\phi=0.05, N=100$ and $\phi=0.25, N=500$, both having a cell length $=20.31 b$. Figure 5.16(b) shows the data for $\phi=0.1, N=1000$ and $\phi=0.25, N=2500$, both having a cell length $=34.73 \mathrm{~b}$. The decrease in the relaxation time scale is evident in from both figures, hence we can conclude that finite-size effects are not responsible. To quantify the rate of decay we compute the relaxation time $\tau_{\kappa}$ as defined in (5.24), but since computing it over the entire range of $\tau$ would require fitting the tails to a known curve we compute

$$
\tau_{\kappa}(1)=\frac{\int_{0}^{1}\langle\tau \overline{\delta \Pi}(0) \overline{\delta \Pi}(\tau)\rangle \mathrm{d} \tau}{\int_{0}^{1}\langle\overline{\delta \Pi}(0) \overline{\delta \Pi}(\tau)\rangle \mathrm{d} \tau}
$$

instead, i.e., the integral is evaluated only up-to $\tau=1$ where the data has not yet become too noisy. This way we can get a more rigorous estimate of the change in time-scale obtained strictly from the simulation data. The values of $\tau_{\kappa}(1)$ for all $\phi$ are shown in Figure 5.17. Evidently the relaxation time decreases rapidly for very small $\phi$ and slowly for larger $\phi$ up to $\phi=0.35$ after which it increases, indicating that there might be competing effects that determine the time scale at small and large $\phi$, respectively.

An explanation for the low- $\phi$ temporal scaling can be had from observing the nature of contributions to the Brownian stress and the process of structural relaxation. The Brownian stress can be separated into a contribution $\Sigma^{B 1}$ from particles in contact, which is of the same form as in hard sphere fluids, and a contribution $\boldsymbol{\Sigma}^{B 2}$ from hydrodynamic interaction between all the particles:

$$
n\left\langle\boldsymbol{S}^{B}\right\rangle=-n^{2} k T a \int_{r=2 b} \hat{\boldsymbol{r}} \hat{\boldsymbol{r}} g(\boldsymbol{r}) \mathrm{d} S+n k T\left\langle\boldsymbol{R}_{S U} \cdot \boldsymbol{R}_{F U}^{-1} \cdot \boldsymbol{\nabla} \ln P_{N}\right\rangle,
$$

where $P_{N}(\boldsymbol{r}, t)$ is the probability density for the $N$-particle configuration [Brady 1993a]. At small volume fractions both $\boldsymbol{\Sigma}^{B 1}$ and $\boldsymbol{\Sigma}^{B 2}$ contribute significantly to the Brownian stress, which implies that the total stress and its fluctuations are determined not only by the particles at contact but also by the configuration of surrounding particles over the distance in which the hydrodynamic interactions decay. The rate of decay of fluctuations in $\Sigma^{B 2}$ is determined by the rate at which the surrounding particles away from contact rearrange into a different configuration. As the volume fraction increases, the number of particles and therefore the number of different configurations that can be sampled in the region around a particle also increases, and consequently the stress gets de-correlated 
faster as new configurations are achieved more easily. This explains the initial decline in $\tau_{\kappa}$ seen in Figure 5.17. On the other hand $g(\boldsymbol{r})$ at contact also increases with increasing $\phi$, and due to the strong lubrication forces between particles at contact the $\Sigma^{B 1}$ contribution becomes more impor$\operatorname{tant}$ than $\boldsymbol{\Sigma}^{B 2}$, thereby diluting the effect of fluctuations in $\boldsymbol{\Sigma}^{B 2}$ on the stress relaxation. The stress relaxation rate is eventually determined by $\Sigma^{B 1}$ only for large volume fractions. The reduced shorttime self-diffusivity of the particles due to stronger hydrodynamic interactions with increasing $\phi$ also slows down the rate at which new configurations can be sampled but evidently it is not the rate-determining factor at small $\phi$. The long-time self-diffusivity does not govern the relaxation rate until 'caging' effects in the microstructure become important, typically for $\phi>0.35$ as observed in our BD simulations.

Thus there are several different competing processes that influence the rate of stress relaxation for small volume fractions: the fluctuations in $\Sigma^{B 2}$ decrease $\tau_{\kappa}$ with increasing $\phi$, while the increasing dominance of $\Sigma^{B 1}$ and the reduction in $D_{0}^{s}$ tend to increase $\tau_{\kappa}$ and eventually win out at $\phi=0.35$, at which point $D_{\infty}^{s}$ also begins to influence the relaxation rate. It's not clear how the combination of these processes can be quantified, so we have approximated the relaxation time scale for $\phi \leq 0.35$ using $\tau_{\kappa}(1)$. For large $\phi$ one would expect the long-time self-diffusivity with hydrodynamic interactions $D_{\infty, H}^{s}(\phi)$ to set the time scale of relaxation but we found that for $0.35 \leq \phi \leq 0.5$ the long-time self-diffusivity without hydrodynamics $D_{\infty, N H}^{s}(\phi)$ provides a better temporal scaling for the simulation data. This does not mean that hydrodynamics are not important in the temporal scaling, rather $D_{\infty, N H}^{s}(\phi)$ being a weaker function of $\phi$ than $D_{\infty, H}^{s}(\phi)$ happens to yield a good approximation for the combined effect of the competing processes discussed above. For $\phi>0.5$ the data scales well with $D_{\infty, H}^{s}(\phi)$. Taking all these considerations into account we define the characteristic diffusivity for stress relaxation with hydrodynamic interactions as

$$
\hat{D}_{H}(\phi)= \begin{cases}\tau_{f i t}(0.05) / \tau_{f i t}(\phi), & 0.05 \leq \phi \leq 0.35 \\ \frac{D_{\infty, N H}^{s}(\phi) \tau_{f i t}(0.05)}{D_{\infty, N H}^{s}(0.35) \tau_{f i t}(0.35)}, & 0.35 \leq \phi \leq 0.5 \\ \frac{D_{0}^{s}(\phi) D_{\infty, N H}^{s}(\phi) \tau_{f i t}(0.05)}{D_{0}^{s}(0.5) D_{\infty, N H}^{s}(0.35) \tau_{f i t}(0.35)}, & \phi>0.5\end{cases}
$$


such that $\hat{D}_{H}(\phi)$ is a continuous function of $\phi$. Here we have used the approximation $D_{\infty, H}^{s}=$ $D_{0}^{s} D_{\infty, N H}^{s}$ [Brady 1994]. The pressure autocorrelation data with long-time tails fitted with the analytical expression for no hydrodynamics is shown in Figure 5.19(a) as a function of the bare diffusive time and in Figure 5.19(b) as a function of the scaled time given by $\hat{\tau}=b^{2} / D \hat{D}_{H}(\phi)$. The analytical curves used for fitting were computed as a function of the scaled time $\hat{\tau}$. The simulation data collapses reasonably well onto a single curve with this scaling. The time-scale for stress relaxation computed using data between $0<\tau \leq 200$ from ASDB-nf simulations with fitted long-time tails is shown in Figure 5.18 and has the expected behavior.

\subsubsection{Simulation results}

The bulk viscosity calculated from the fitted simulation data is shown in Figure 5.20 for both the SD and ASDB-nf simulations, along with the scaled theoretical curve given by (5.32) with $\hat{D}_{H}(\phi)$ from (5.41). The discrepancy in the SD results for $\phi>0.45$ is due to the severe finite-size effects in the simulations with only 27 particles. The high-frequency bulk modulus computed from the zero-time limit of the pressure autocorrelation function is shown in Figure 5.21 along with the scaled theoretical prediction given by (5.34). The scaled theory provides a good approximation for the simulation results. It underestimates the bulk modulus by a constant factor although it has the correct scaling with $\phi$. The discrepancy in theory and simulation results is because the extrapolation of the dilute theory to account for hydrodynamic interactions for all $\phi$ using $D_{0}^{s}$ is not exact, as previously shown by Lionberger and Russel [1994].

We also computed the shear stress autocorrelation function and the shear viscosity, for which the values are known from both simulation and experiments for a wide range of volume fractions. This serves as an additional check on our simulations. The raw data for the nondimensional shear stress autocorrelation function $C_{H}^{S}$ is shown in Figure 5.22. It is able to capture the $t^{-7 / 2}$ long-time decay of the shear stress autocorrelation before degenerating to noise, as the long-time limit is reached much earlier than in the pressure autocorrelation. This can be attributed to the monopolar nature of the decay of pressure fluctuations which results in a slow temporal decay, while the shear stress exhibits a faster quadrupolar decay. We tried two different scalings for the magnitude of $C_{H}^{S}$ using 
the same arguments used for scaling $C_{H}^{P}$ :

$$
\begin{aligned}
\overline{C_{H, D}^{S}} & =\frac{N D_{0}^{S}(\phi)}{\phi g_{0}(2 ; \phi)} C_{H}^{S}, \\
\overline{C_{H, \eta}^{S}} & =\frac{N}{\phi g_{0}(2 ; \phi) \eta_{\infty}^{\prime}(\phi)} C_{H}^{S},
\end{aligned}
$$

(shown in Figure 5.23 (a) and (b), respectively) where $\eta_{\infty}^{\prime}$ is the high-frequency shear viscosity nondimensionalized with the fluid viscosity. The analytical curve without hydrodynamics is also shown and clearly matches with the simulation data in the long-time limit. Looking at the short time limit in the figure, it is evident that $\eta_{\infty}^{\prime}$ provides a more accurate scaling for $C_{H}^{S}$ compared to scaling with $D_{0}^{s}$. Although the generalized Stokes-Einstein relation 5.38 holds over a wide range of concentrations, the agreement is not exact [Banchio et al. 1999]. This is likely because $D_{0}^{s}$ is the inverse of the trace of $\boldsymbol{R}_{F U}$ so it gives a good approximation for the scaling of the trace of $\boldsymbol{R}_{S U}$ (pressure moment), which is essentially the first moment of the forces obtained from $\boldsymbol{R}_{F U}$. Hence $D_{0}^{s}$ is the appropriate scaling for $C_{H}^{P}$. In contrast, $\eta_{\infty}^{\prime}$ is computed as an average of the deviatoric stress components from $\boldsymbol{R}_{S E}$ over an ensemble of equilibrium particle configurations in a linear shear flow. Therefore it provides the best scaling for the strength of hydrodynamic interactions corresponding to the deviatoric (shear) stress. This observation further supports the theory that the $D_{0}^{s}(\phi)$ scaling in the bulk viscosity comes primarily from the scaling of the strength of hydrodynamic interactions.

The slowing down of the relaxation rate for $\phi>0.35$ is evident from the $C_{H}^{S}$ data but the faster rate of decay for smaller $\phi$ is very diminished, although it is present. The rate of relaxation of the shear stress is much faster than the pressure because of its quadrupolar nature, so the fluctuations in $\boldsymbol{\Sigma}^{B 2}$ are not fast enough to make a qualitative difference in the long time decay. The effect of long-range fluctuations is more pronounced in the pressure relaxation because it is much slower due to its monopolar nature. The behavior at high volume fractions is the same for both. Accordingly we use the same temporal scaling for the shear stress as for the pressure autocorrelation given by (5.41) but with $\tau_{f i t}(\phi)=1$, thus ignoring the slight variations in the rate of decay for smaller volume fractions. The scaled and fitted simulation data for $\overline{C_{H}^{S}}$ is shown in Figure 5.24. All the curves for high $\phi$ collapse very nicely onto a single curve and there is a slight variation in the temporal scaling for small $\phi$ as expected. The Brownian shear viscosity computed from this data is shown in Figure 5.25, along with the simulation results of Foss and Brady [1999], experimental results of Segrè et al. 
[1995], and experimental results of Cheng et al. [2002]. The shear viscosity from BD simulations is also shown to demonstrate the effect of hydrodynamic interactions, especially the increase in the shear viscosity due to lubrication interactions at high $\phi$. All the data match very nicely all the way up-to the highest volume fraction studied, $\phi=0.55$. The scaled dilute theory is also shown and it does an excellent job of predicting the shear viscosity for all $\phi$.

Finally, the high-frequency bulk viscosity which gives the direct hydrodynamic contribution was computed according to (5.4) by averaging over an ensemble of equilibrium configurations. The direct hydrodynamic stress is given by

$$
\left\langle\boldsymbol{S}^{E}\right\rangle=-\left\langle\boldsymbol{R}_{S U} \cdot \boldsymbol{R}_{F U}^{-1} \cdot \boldsymbol{R}_{F E}-\boldsymbol{R}_{S E}\right\rangle:\langle\boldsymbol{e}\rangle,
$$

where the $\boldsymbol{R}_{S E}$ resistance tensor includes the new hydrodynamic functions that give the trace of the stress due to an imposed expansion flow, and $\boldsymbol{R}_{S U}$ includes the functions for computing the disturbance pressure due to the motion of other particles. The stress was computed by imposing a uniform rate of expansion for all particles in a given configuration without actually simulating their motion. It was averaged over 300 independent equilibrium configurations of 1000 particles for each volume fraction to get the average bulk stress in an equilibrium suspension due to the imposed expansion flow. The configurations used were taken at regular intervals from the equilibrium ASDBnf simulations for computing the stress autocorrelations, thus ensuring that all the configurations were properly equilibrated. The high-frequency bulk viscosity with the single particle contribution subtracted from it $\left(\kappa^{H}=\kappa_{\infty}^{\prime}-\frac{4}{3} \eta \phi\right)$ is shown in Figure 5.26. The simplest scaling would suggest that since $\kappa^{H}$ is a particle-particle contribution it should scale as $\phi^{2}$. Additionally, since it is a measure of the pressure moment in an equilibrium configuration the scaling for higher volume fractions must be closest to the equilibrium osmotic pressure $\bar{\Pi}_{0}$, which would account for the increase in the particle pressure with $\phi$. Although the equilibrium osmotic pressure in a suspension is a thermodynamic property, it can be determined from purely hydrodynamic considerations along with the scale factor of $k T$ as shown by Brady [1993a]. Therefore the equilibrium osmotic pressure should the same scaling with volume fraction as the pressure moment due to an imposed flow. Thus rescaling the dilute theory for the direct hydrodynamic contribution to the bulk viscosity with the 
osmotic pressure we get

$$
\kappa^{H}=1.57 \phi^{2} \bar{\Pi}_{0}(\phi)
$$

and as seen on the figure this simple scaling agrees remarkably well with the simulation results.

The total contribution to the bulk viscosity due to interactions between particles is shown in Figure 5.27. This along with the single particle bulk viscosity completes all the contributions to the bulk viscosity in the linear response regime of small $\mathrm{Pe}$ for all concentrations with full hydrodynamic interactions. The hydrodynamic contribution is consistently smaller than the Brownian contribution and at higher volume fractions the Brownian bulk viscosity diverges much faster with $\phi$. The Brownian contribution to both the bulk and the shear viscosity scales as $g_{0}(2 ; \phi) / D_{\infty, H}^{S}(\phi) D_{0}^{S}(\phi)$ and therefore has a stronger divergence with increasing $\phi$ as seen in Figure 5.20 and Figure 5.25. Recent experimental work by Cheng et al. [2002], also shown on the plots, suggests an exponential divergence of the shear viscosity for higher concentrations in the glassy region. We expect the Brownian bulk viscosity to also exhibit a similar behavior.

\subsection{Conclusions}

The equilibrium properties of suspensions can be used to get significant insight into the physical processes that govern their rheological behavior. This chapter describes the equilibrium simulations that were performed to study bulk viscosity effects in suspensions in the linear response limit for all concentrations.

The Brownian Dynamics simulations we performed gave significant insight into the time scale of stress relaxation. With only the thermodynamic and microstructural influences governing the relaxation time scale it became apparent that there is no universal diffusive scaling for all volume fractions. The long-time self-diffusivity governs the time scale of relaxation only at high volume fractions when it is necessary for particles to break out of a cage or move a distance comparable to their size to relax the microstructure into a different configuration. At small volume fractions different configurations can be achieved without the particles having to move a significant distance. The microstructure is relaxed primarily by particle collisions taking place at the bare diffusive time scale.

The BD simulations also highlight that care must be taken when simulating processes that have 
a long time-scale for decay as the slow decay may not be captured in the simulations before the data reduces to noise. We were able to deduce the correct decay of the autocorrelation functions from viscoelastic theory and use it to compute the bulk viscosity. With knowledge of the correct scaling we were able to predict the bulk and shear viscosities within reasonable accuracy by scaling up the dilute theory expressions. Simulations with larger systems could be used to get more accurate results, but one must be careful with very large systems at high volume fractions as they have been known to crystallize faster than smaller systems and so may not give the correct temporal scaling for metastable hard sphere fluids [Rintoul and Torquato 1996]. For very high volume fractions it might be more appropriate to determine the rheological properties from non-equilibrium simulations because the time scale of decay of the stress autocorrelation functions becomes very large.

The inclusion of hydrodynamic interactions introduces several new factors that affect the time scale of relaxation significantly. The pressure autocorrelation data highlights the competition between long-range structural relaxation and the slowing down of the relaxation due to hydrodynamic interactions, which was not noticeable in the shear stress autocorrelation. These factors govern the relaxation time scale at small $\phi$ but eventually at high concentrations it is the long-time self diffusivity that governs the structural relaxation. In the intermediate regime there is a competition between several processes and so there is no single diffusivity that defines the relaxation time for all concentrations.

Finally it must be pointed out that the monopolar nature of the pressure fluctuations is responsible for its slow decay, with the consequence that there is more variability in the temporal scaling as other processes get an opportunity to affect the time scale. The monopolar nature also results in higher values of the bulk viscosity compared to the shear viscosity as it takes longer to dissipate the fluctuations in pressure than those in the shear stress. Thus one cannot simply approximate the bulk viscosity with the shear viscosity values as doing so will underestimate bulk viscosity effects in the suspension flow. The bulk viscosity term is quite significant and it must be included in the simulation of suspension flows when there are variations in the particle concentration. 


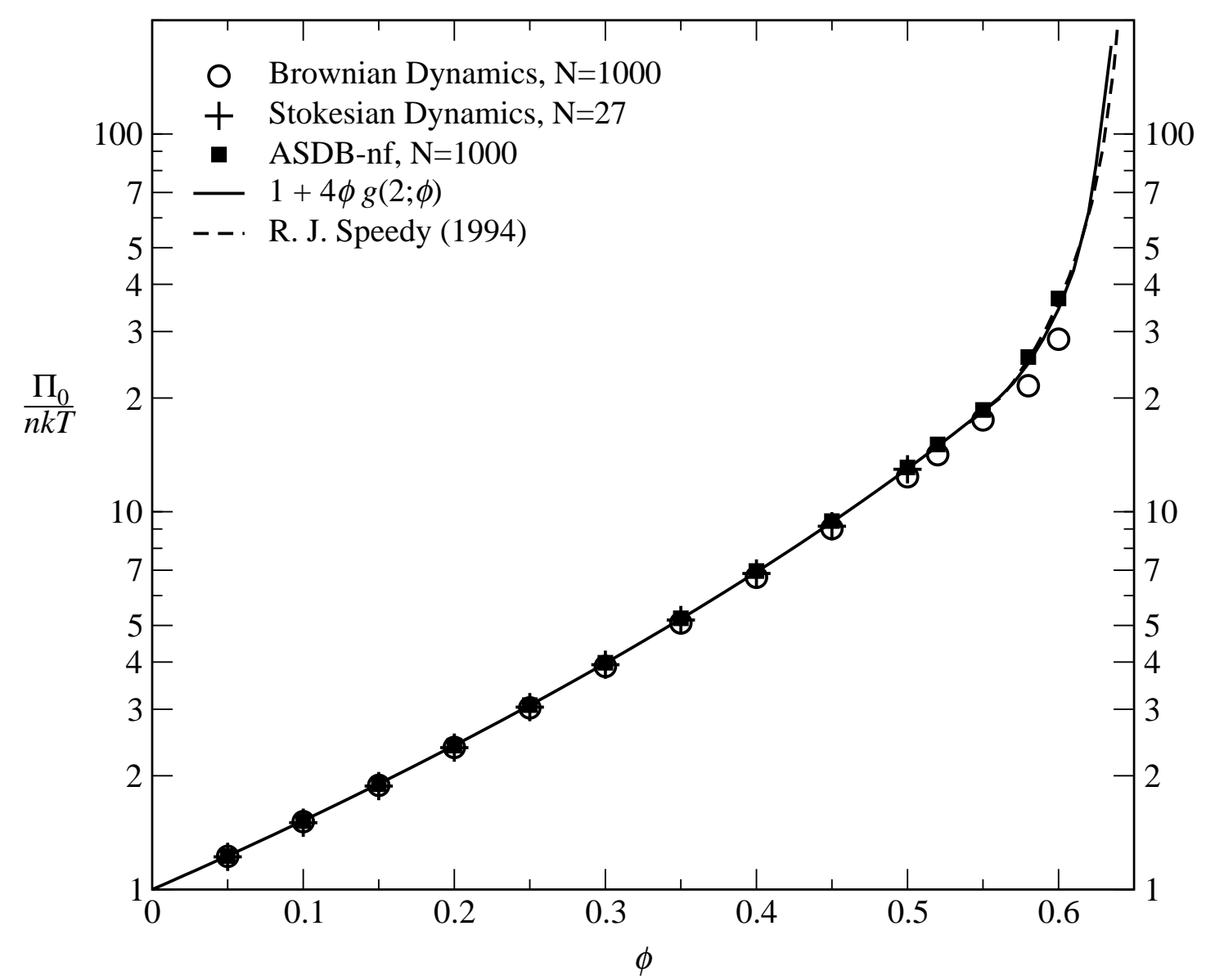

Figure 5.1: The equilibrium osmotic pressure $\Pi_{0}$ determined from Brownian Dynamics (open circles) and Accelerated Stokesian Dynamics - near field simulations (black squares) nondimensionalized with $n k T$, as a function of the volume fraction $\phi$. The data was averaged over all the configurations occurring in the equilibrium simulations for each $\phi$ for each of the simulation methods $\left(\sim 10^{6}\right.$ realizations). The theoretical value given by $\overline{\Pi_{0}}=1+4 \phi g_{0}(2 ; \phi)$ is also shown with $g_{0}(2 ; \phi)$ data from Rintoul and Torquato [1996] for the $\phi>0.55$. The pressure data from Speedy [1994] is also shown for the metastable and glassy region 


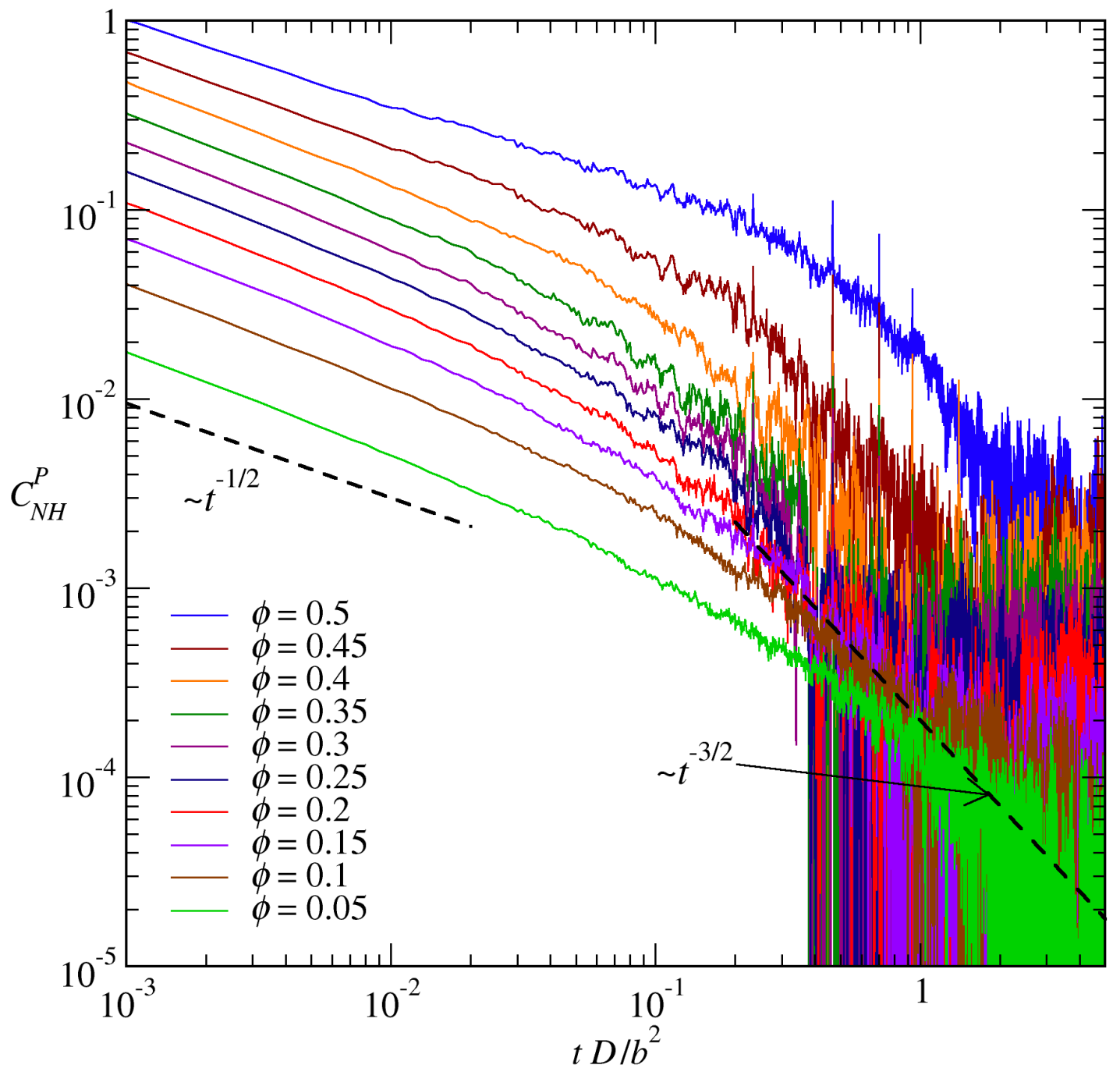

Figure 5.2: Pressure autocorrelation function from Brownian Dynamics with $N=100$ particles as a function of the diffusive time 


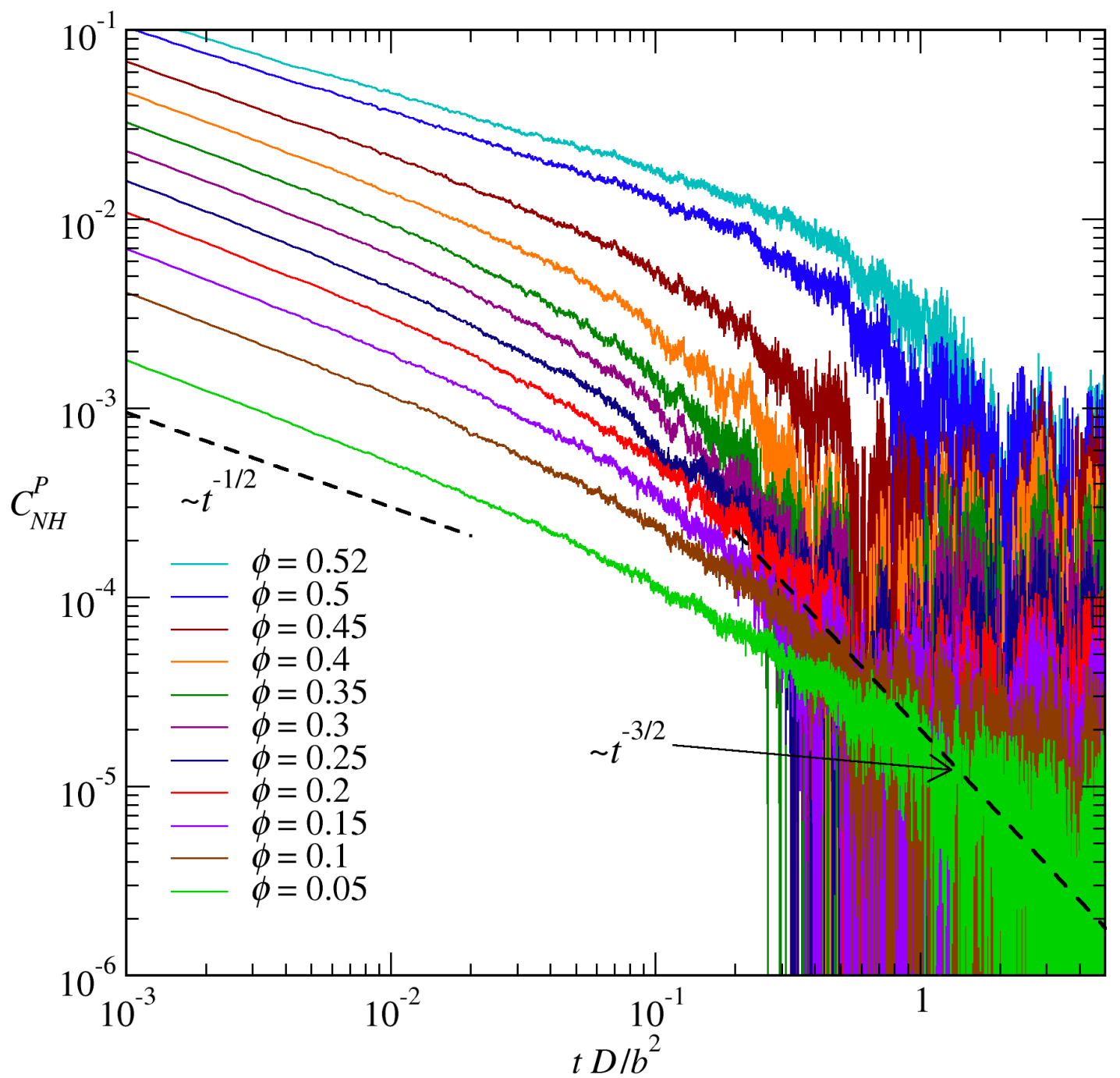

Figure 5.3: Pressure autocorrelation function from Brownian Dynamics with $N=1000$ particles as a function of the diffusive time. 

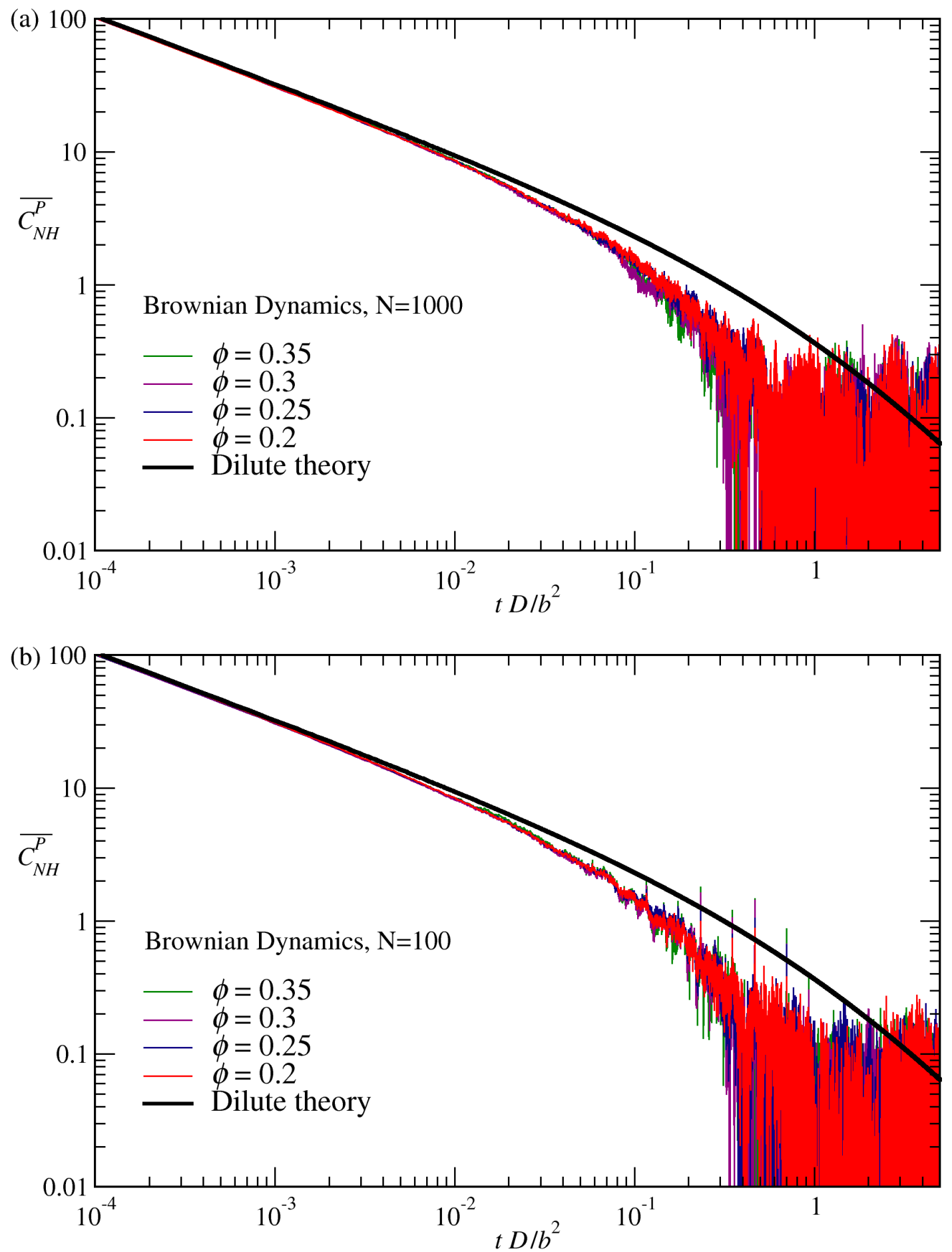

Figure 5.4: Comparison of the scaled pressure autocorrelation function $\overline{C_{N H}^{P}}=C_{N H}^{P} N /\left(\phi g_{0}(2 ; \phi)\right)$ (5.22) from Brownian Dynamics with (a) $N=1000$ and (b) $N=100$ particles as a function of the diffusive time for intermediate volume fractions. The plots illustrate the faster decay of pressure fluctuations for $N=100$ due to finite-cell-size effects. For $N=1000$ the data is aligned very closely with the analytical curve. 

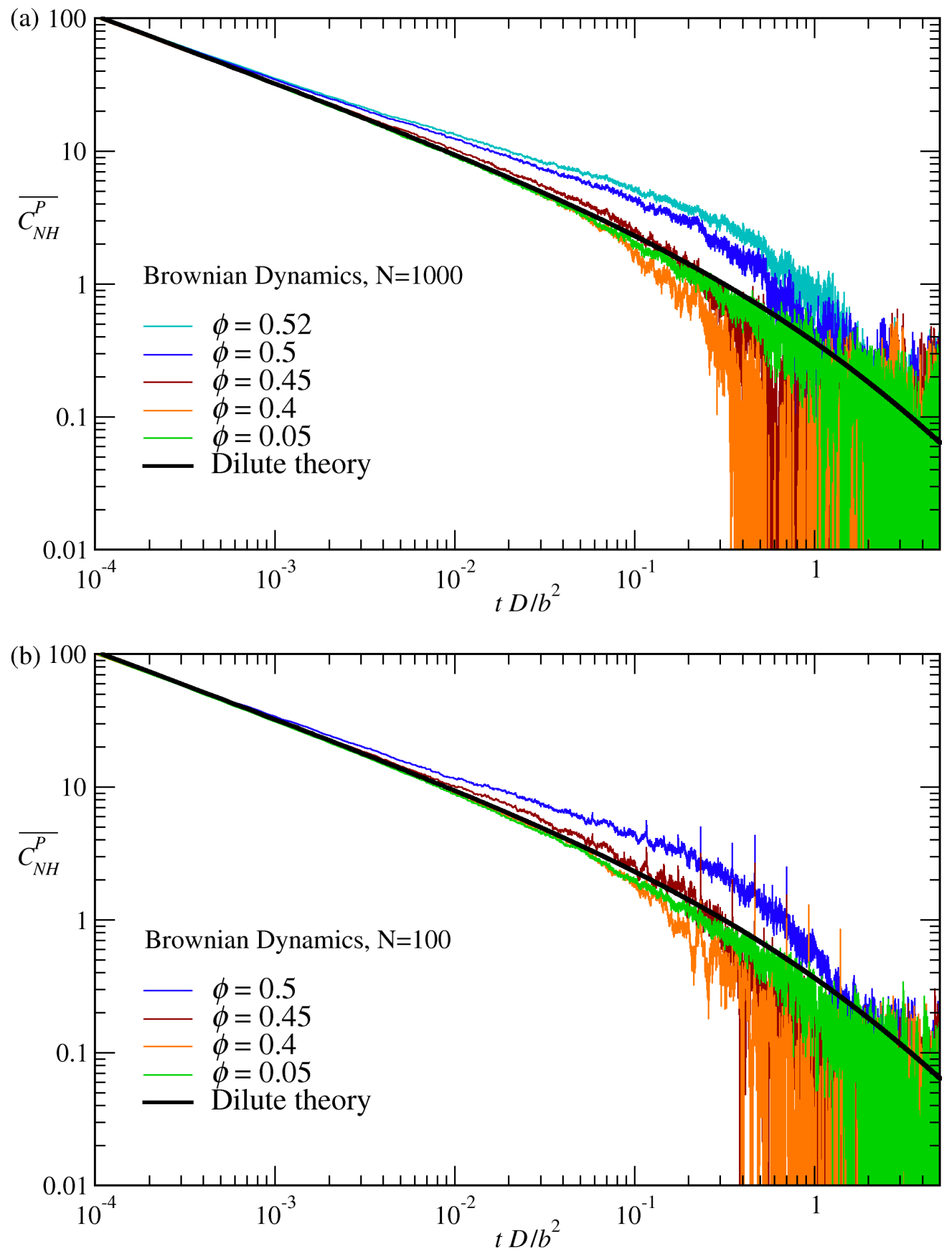

Figure 5.5: Comparison of the scaled pressure autocorrelation function $\overline{C_{N H}^{P}}=C_{N H}^{P} N /\left(\phi g_{0}(2 ; \phi)\right)$ (5.22) from Brownian Dynamics with (a) $N=1000$ and (b) $N=100$ particles as a function of the diffusive time for high volume fractions. In both cases the time-scale of decay increases with increasing volume fraction for $\phi \geq 0.4$. The data for $\phi=0.05$ is also shown to demonstrate that for low $\phi$ (largest cell size simulated) the simulation data matches perfectly with the predicted theoretical curve. 


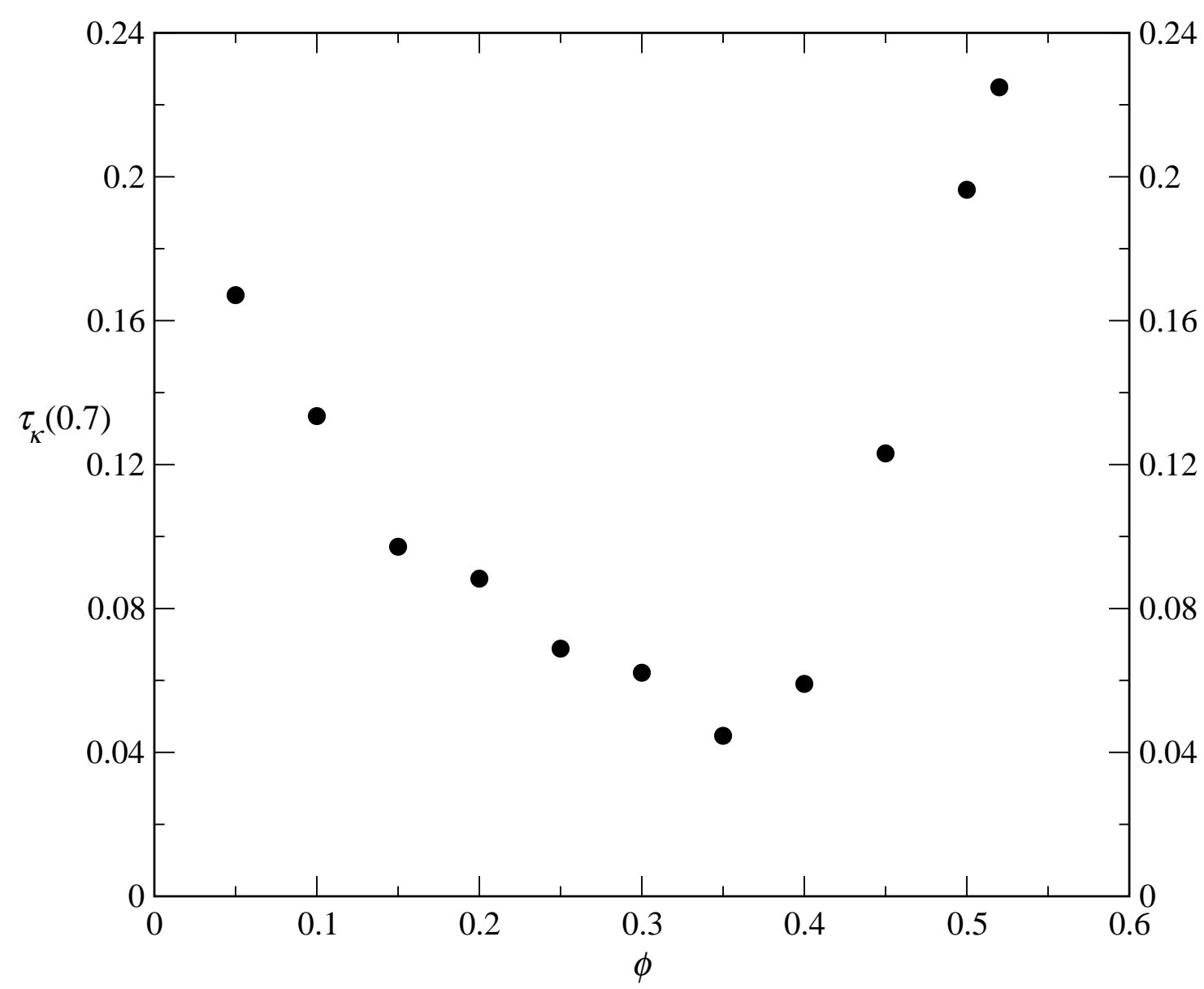

Figure 5.6: Stress relaxation time from data between $0<\tau \leq 0.7$ for the pressure autocorrelation function from BD simulations with $N=1000$. 


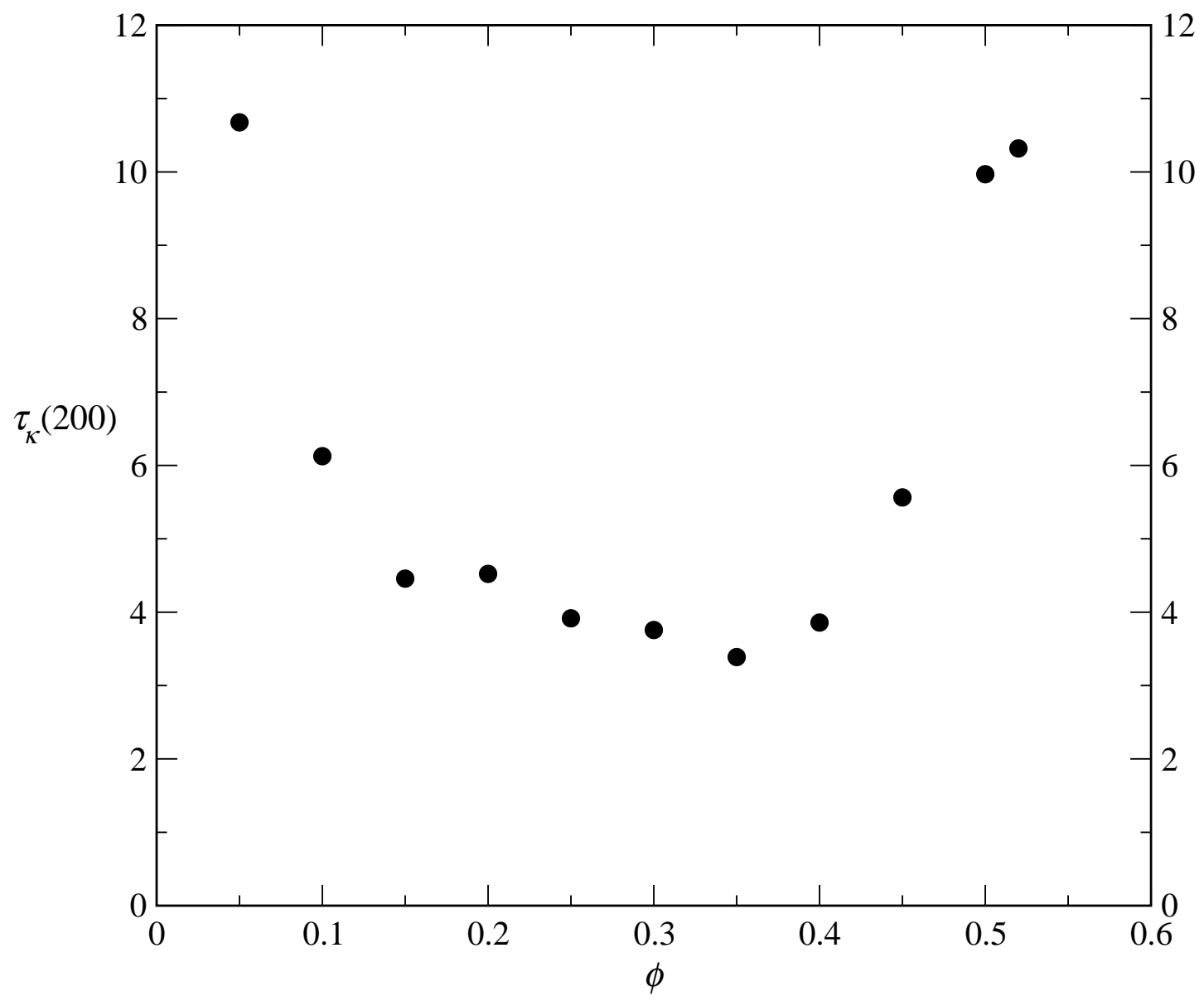

Figure 5.7: Stress relaxation time from the data with fitted long-time tails between $0<\tau \leq 200$ for the pressure autocorrelation function from BD simulations with $N=1000$. 

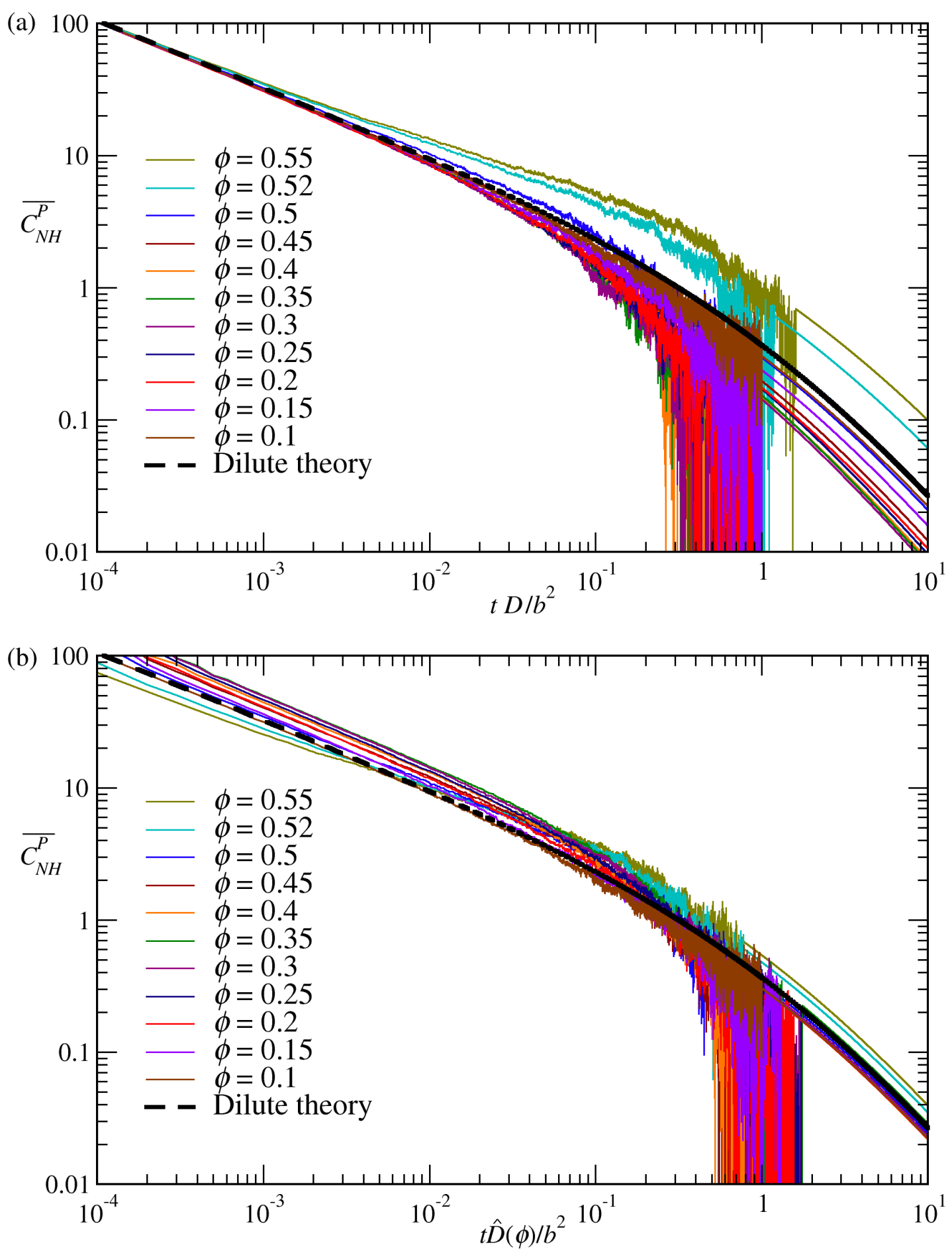

Figure 5.8: The scaled Pressure autocorrelation function from Brownian Dynamics $\overline{C_{N H}^{P}}=$ $C_{N H}^{P} N /\left(\phi g_{0}(2 ; \phi)\right)(5.22)$ with $N=1000$ particles with fitted long-time tails for all volume fractions simulated, as a function of the diffusive time. In plot (a) time is scaled with the bare diffusivity of he particles and in (b) with the characteristic diffusivity $\hat{D}(\phi)$. The latter scaling collapses all the long-time tails onto the predicted theoretical curve. 


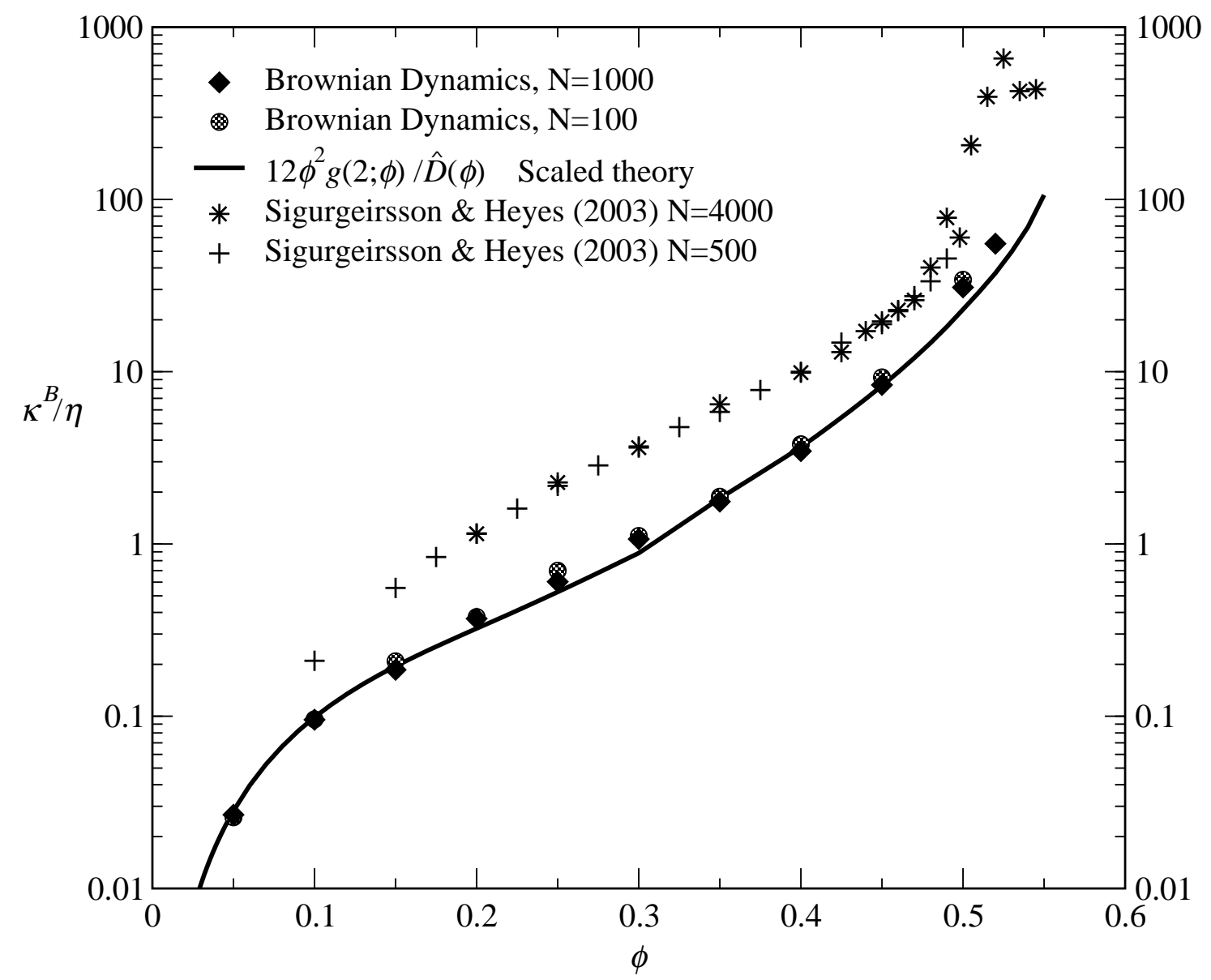

Figure 5.9: The Green-Kubo bulk viscosity $\overline{\kappa^{B}}$ from equilibrium Brownian Dynamics simulations (solid diamonds), the MD simulation results of Sigurgeirsson and Heyes [2003] for the bulk viscosity of hard sphere fluids ( + and $*$ ), and the scaled theoretical curve (solid line). 


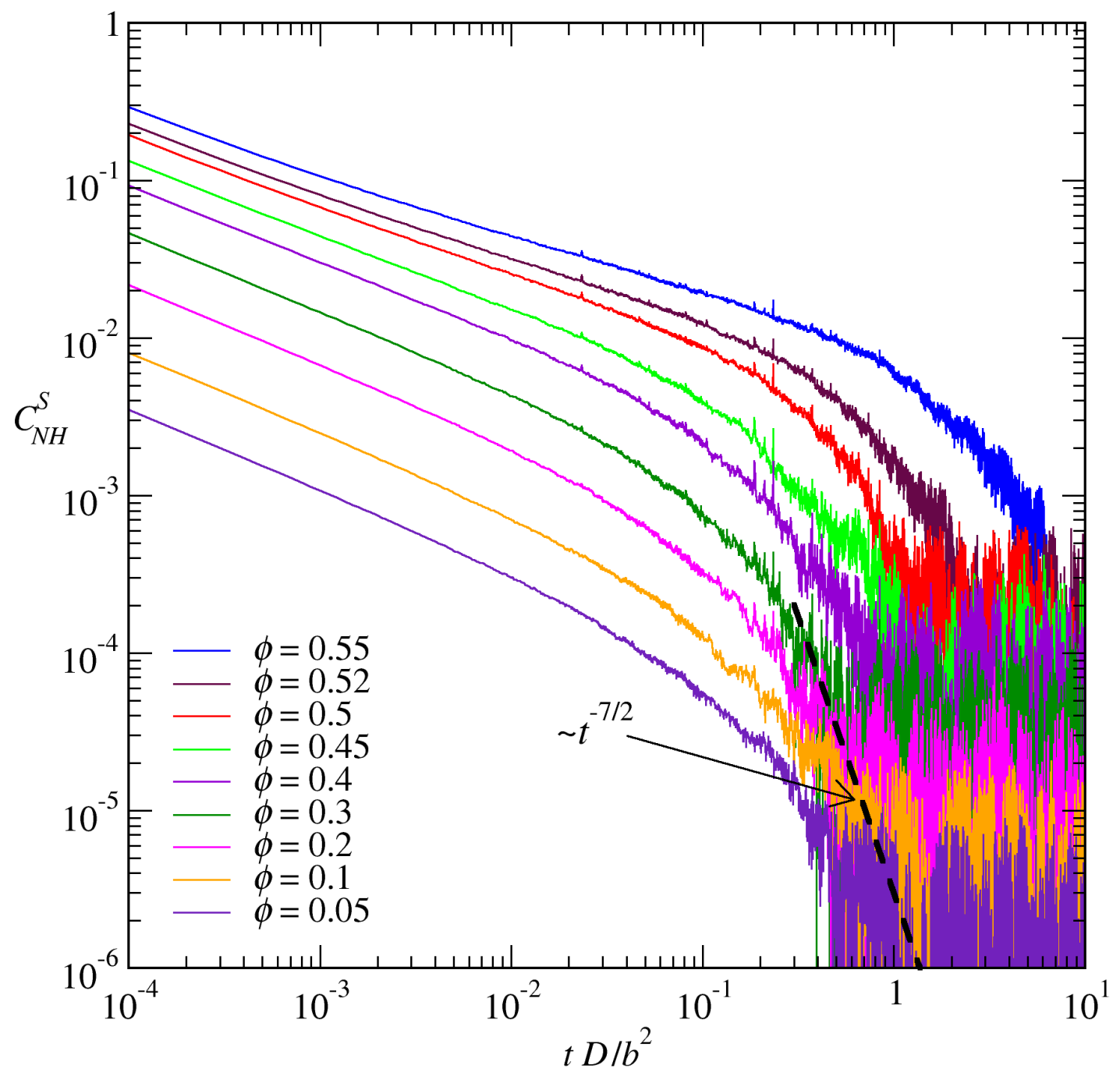

Figure 5.10: Shear stress autocorrelation function from Brownian Dynamics with $N=1000$ particles as a function of the diffusive time. 

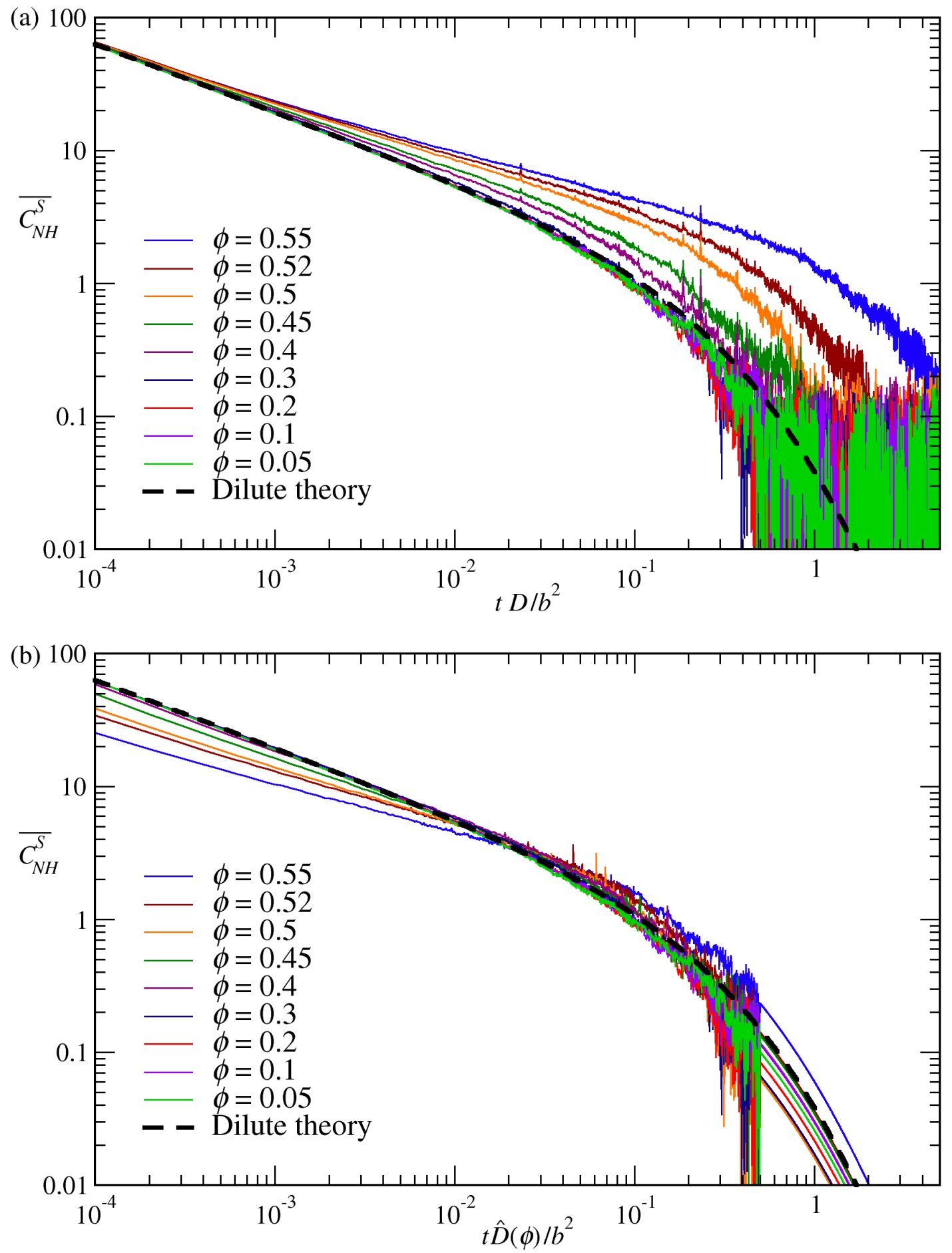

Figure 5.11: The scaled Shear stress autocorrelation function $\overline{C_{N H}^{S}}=C_{N H}^{S} N /\left(\phi g_{0}(2 ; \phi)\right)$ from Brownian Dynamics with $N=1000$ particles with fitted long-time tails in (b) for all volume fractions simulated, as a function of the diffusive time. In plot (a) time is scaled with the bare diffusivity of he particles and in (b) with the characteristic diffusivity $\hat{D}(\phi)$. The latter scaling collapses all the long-time tails onto the predicted theoretical curve. 


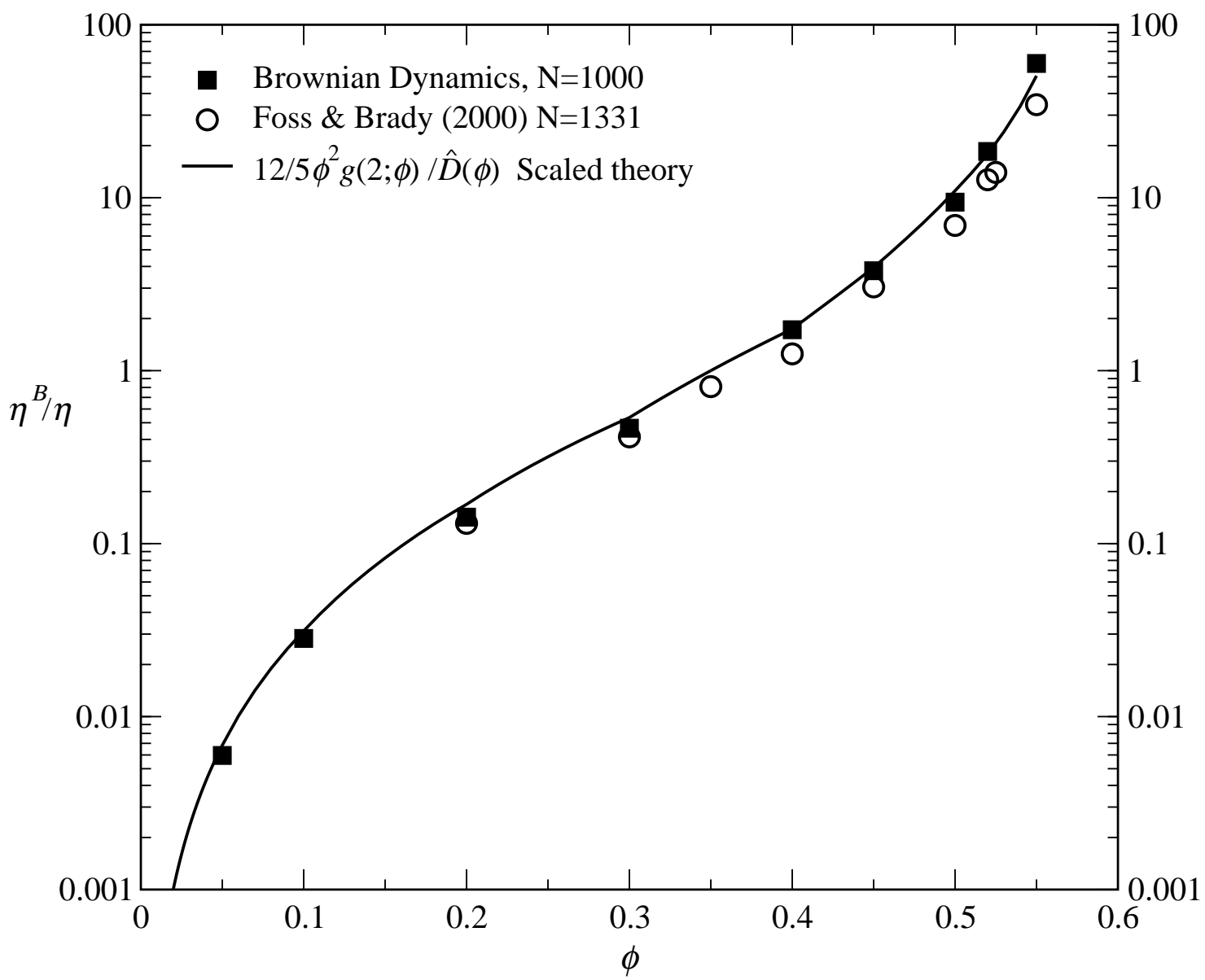

Figure 5.12: The zero-shear viscosity $\eta^{B}$ from equilibrium Brownian Dynamics simulations (solid diamonds), the simulation results of Foss and Brady [2000] for $\eta^{B}$ (open circles), and the scaled theoretical curve (solid line). The simulation data was obtained from equilibrium simulations with a total of $\sim 2 \times 10^{7}$ time-steps for each $\phi$. 


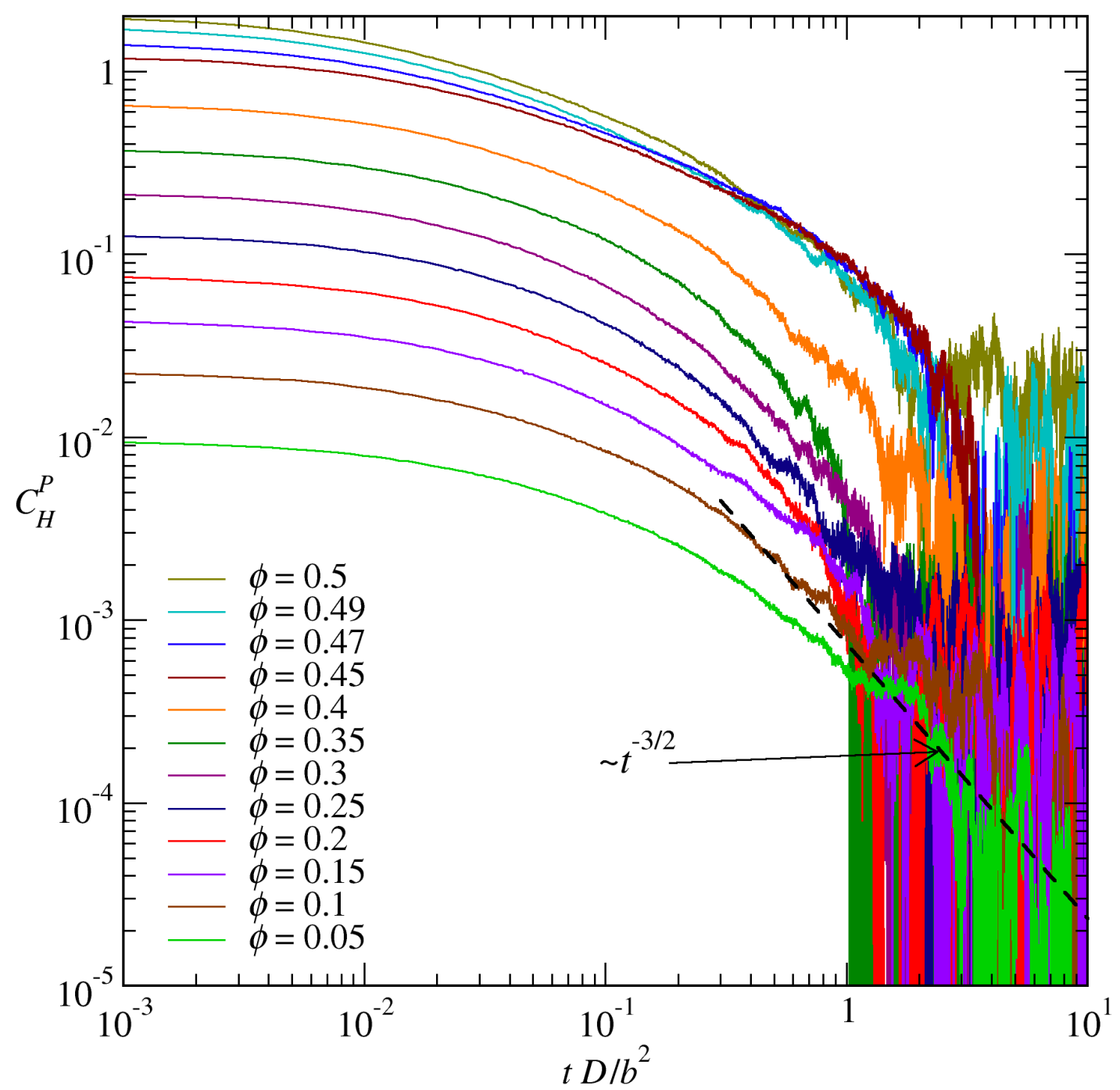

Figure 5.13: Pressure autocorrelation function from Stokesian Dynamics simulations with $N=27$ particles as a function of the diffusive time. For $\phi>0.45$ the decay is exponential as the simulation cell size becomes small enough that the periodic boundary conditions have the effect of diffusion in a finite box. 


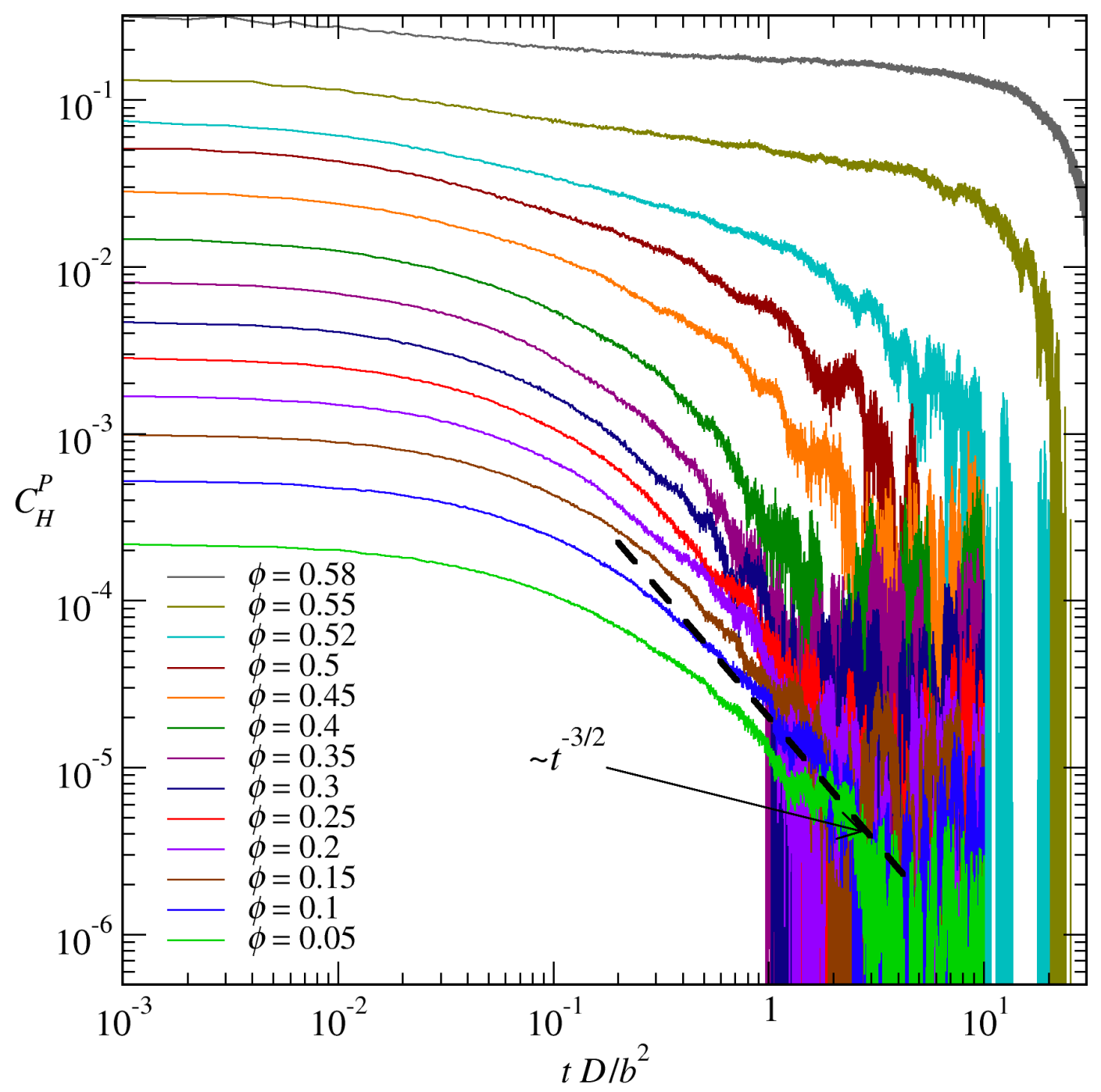

Figure 5.14: Pressure autocorrelation function from Accelerated Stokesian Dynamics - near field simulations with $N=1000$ particles as a function of the diffusive time. For $\phi>0.52, C_{H}^{P}$ decays much more slowly because the particles are in the glassy regime. At long times the finite size of the simulation cell causes the pressure autocorrelation to decay exponentially as the periodic boundary conditions simulate diffusion of the particles in a finite box. 

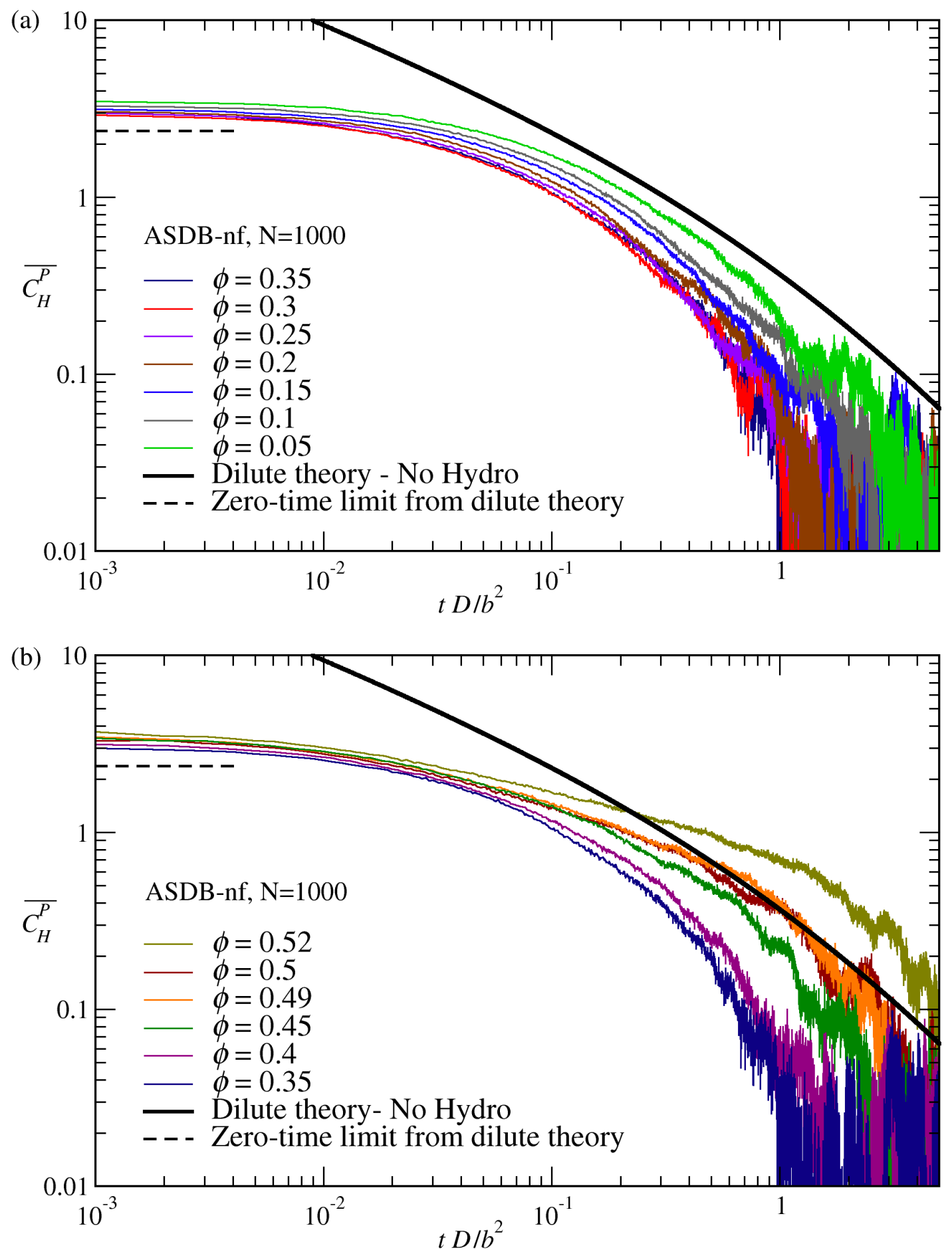

Figure 5.15: Scaled pressure autocorrelation function from ASDB-nf simulations $\overline{C_{H}^{P}}=$ $C_{H}^{P} N D_{0}^{s}(\phi) / \phi g_{0}(2 ; \phi)$ with $N=1000$ particles as a function of the diffusive time, for (a) $0.05 \leq$ $\phi \leq 0.35$ and (b) $0.35 \leq \phi \leq 0.52$. For the lower volume fractions the rate of decay decreases as $\phi$ increases while for $\phi \geq 0.35$ the rate of decay increases with $\phi$. The analytical curve for no hydrodynamics is shown by the black curve and has the same long-time behavior as the simulation data. Also shown is the zero-time limit from scaled dilute theory. 

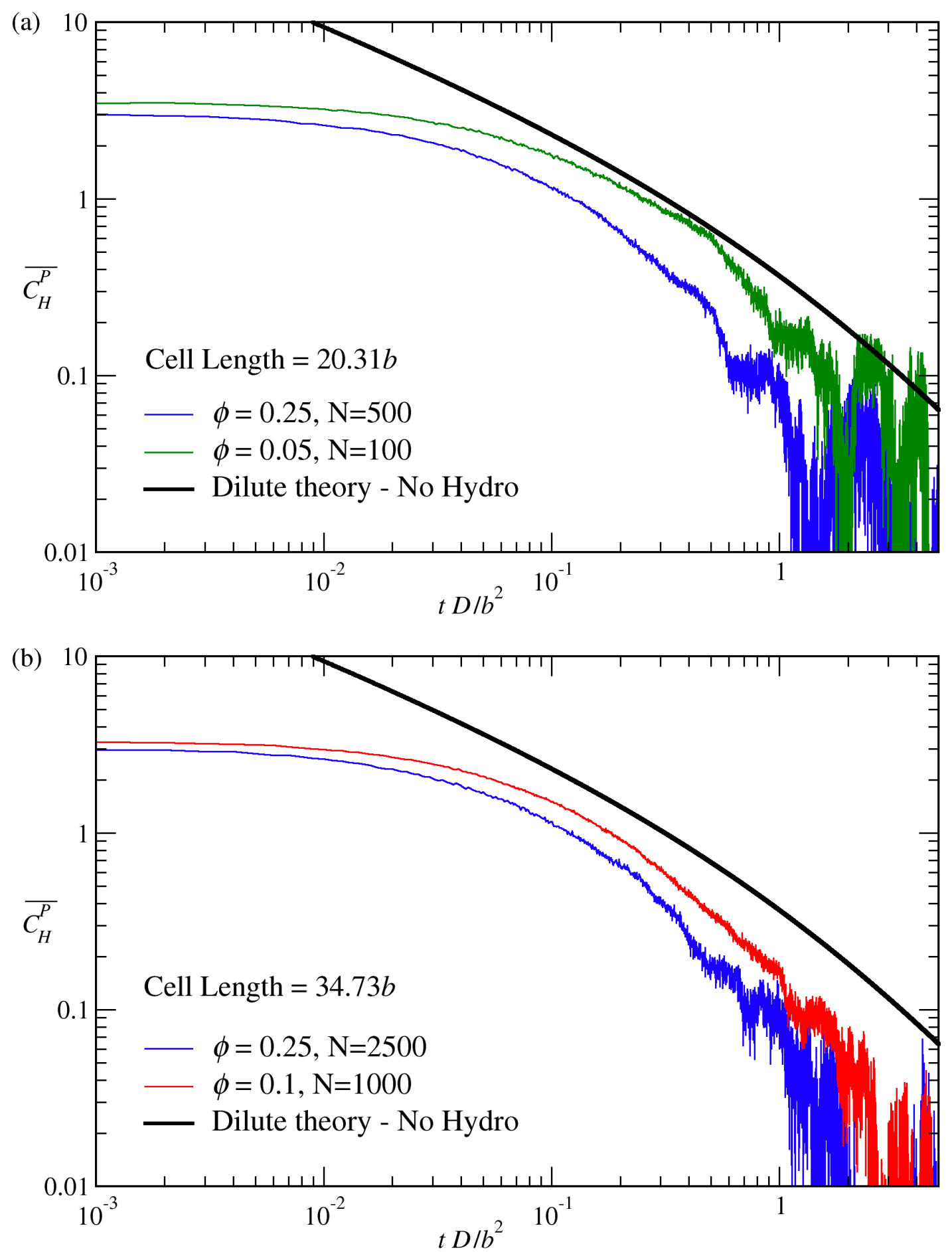

Figure 5.16: Comparison of the scaled pressure autocorrelation function from ASDB-nf simulations $\overline{C_{H}^{P}}=C_{H}^{P} N D_{0}^{s}(\phi) / \phi g_{0}(2 ; \phi)$ with the same cell size given by (a) $L=20.31 b$ and (b) $L=34.73 b$ but for different volume fractions. The decrease in the rate of decay with increasing $\phi$ for $\phi<0.35$ is evident in both plots, indicating that it is not a finite-size effect. 


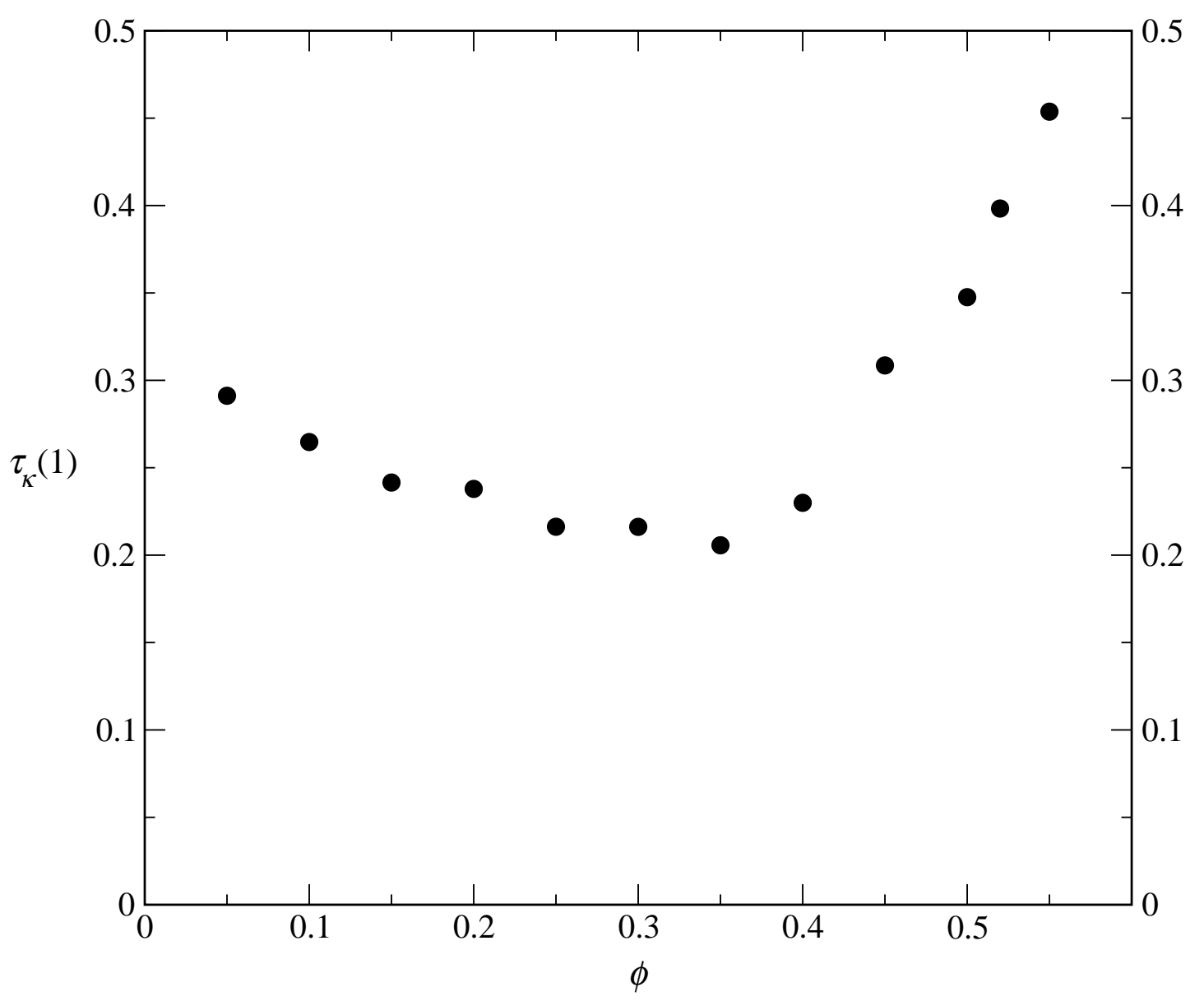

Figure 5.17: Stress relaxation time from data between $0<\tau \leq 1$ for the pressure autocorrelation function from ASDB-nf simulations with $N=1000$. 


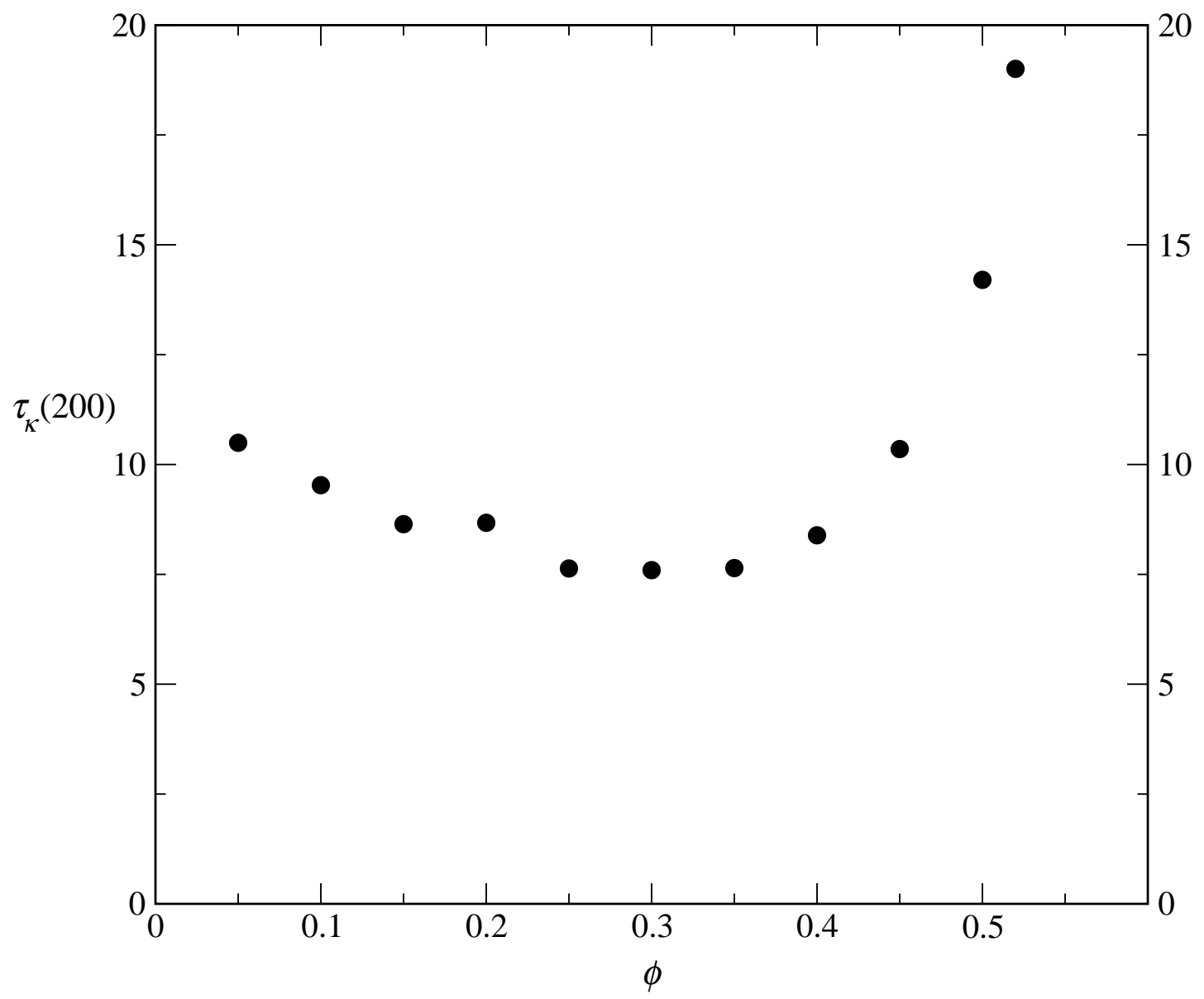

Figure 5.18: Stress relaxation time from data between $0<\tau \leq 200$ with fitted long-time tails for the pressure autocorrelation function from ASDB-nf simulations with $N=1000$. 

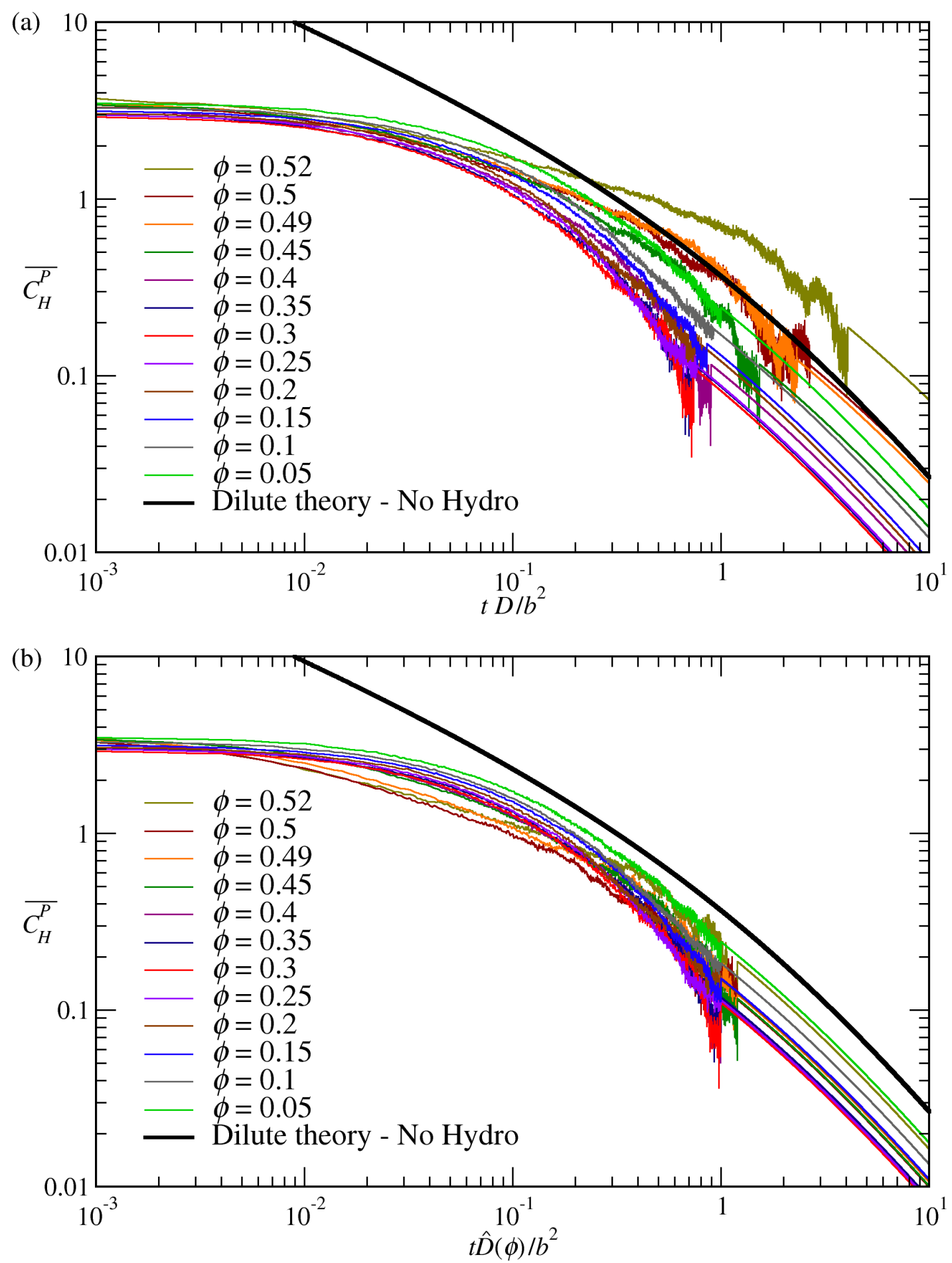

Figure 5.19: Scaled Pressure autocorrelation function from ASDB-nf simulations $\overline{C_{H}^{P}}=$ $C_{H}^{P} N D_{0}^{s}(\phi) / \phi g_{0}(2 ; \phi)$ with $N=1000$ particles for all $\phi$ simulated, as a function of (a) the diffusive time and (b) diffusive time scaled with the stress relaxation time-scale. The long-time tails were obtained by fitting the analytical curve for no hydrodynamics (also shown) with the corresponding temporal scaling. 


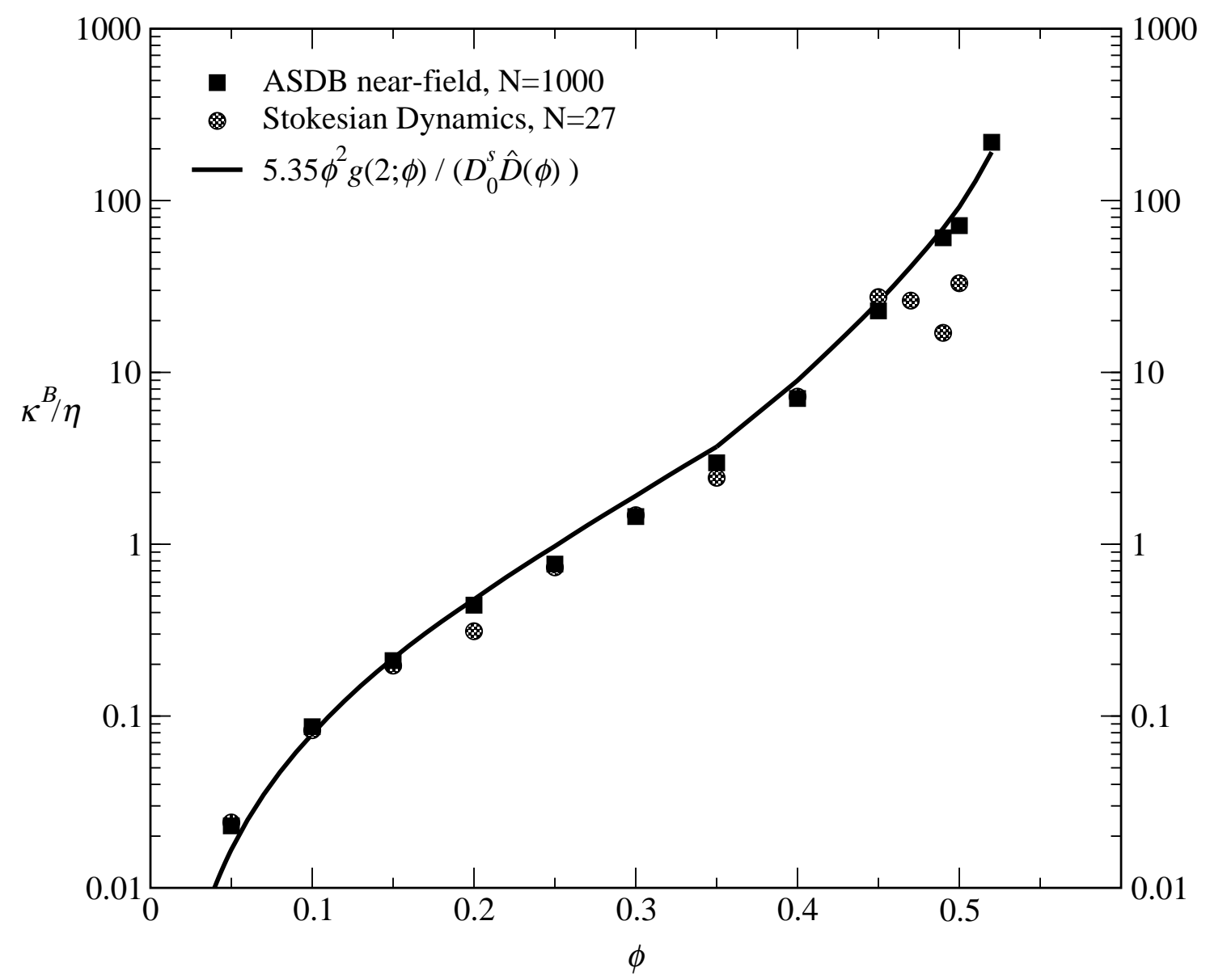

Figure 5.20: The Green-Kubo bulk viscosity $\overline{\kappa^{B}}$ as a function of volume fraction from equilibrium ASDB-nf simulations (solid squares) with $N=1000$, SD simulations (shaded circles) with $N=27$, and the scaled dilute theory (solid line). 


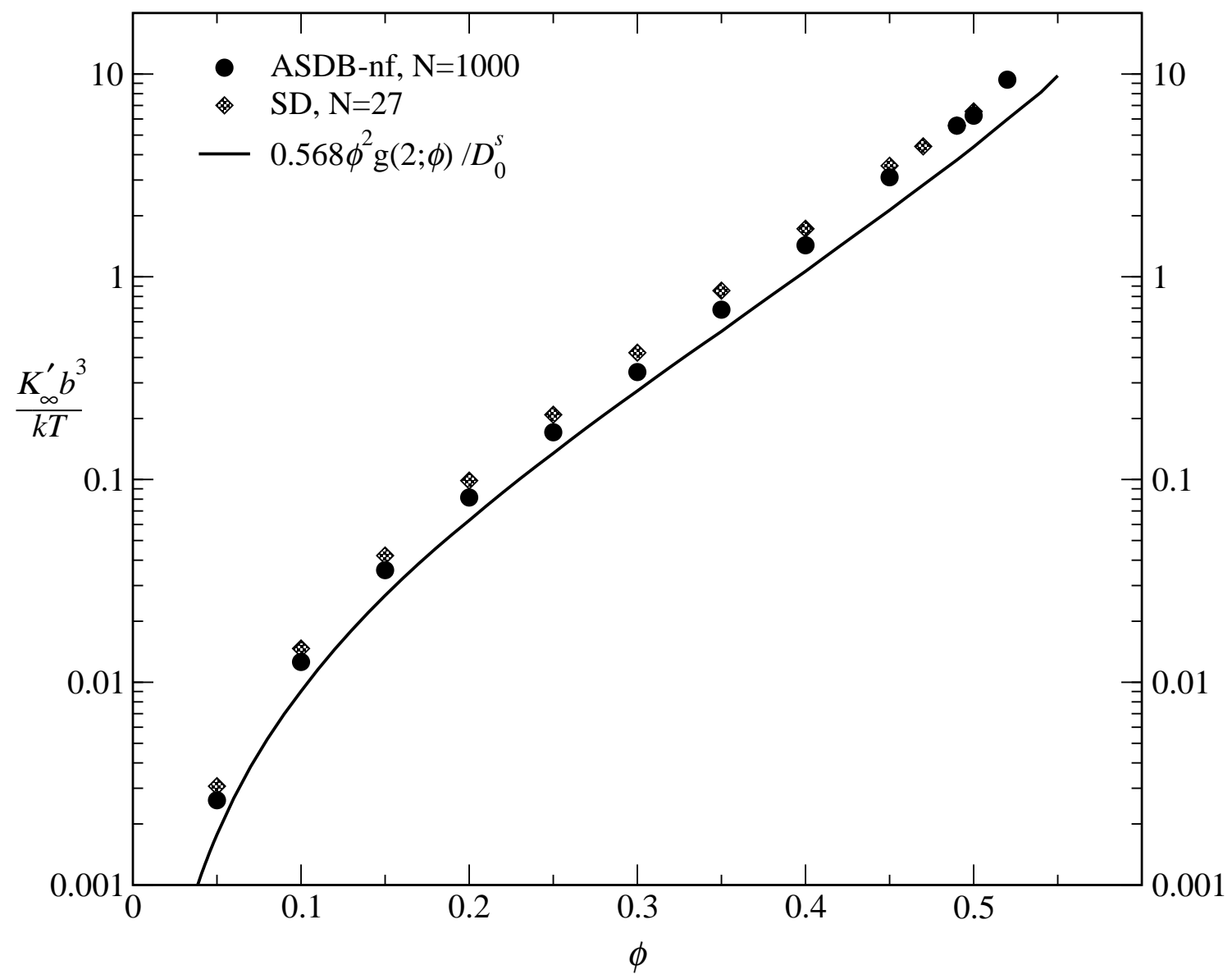

Figure 5.21: The high-frequency bulk modulus as a function of volume fraction from equilibrium ASDB-nf simulations (solid squares) with $N=1000$, SD simulations (shaded circles) with $N=27$, and the scaled dilute theory (solid line). 


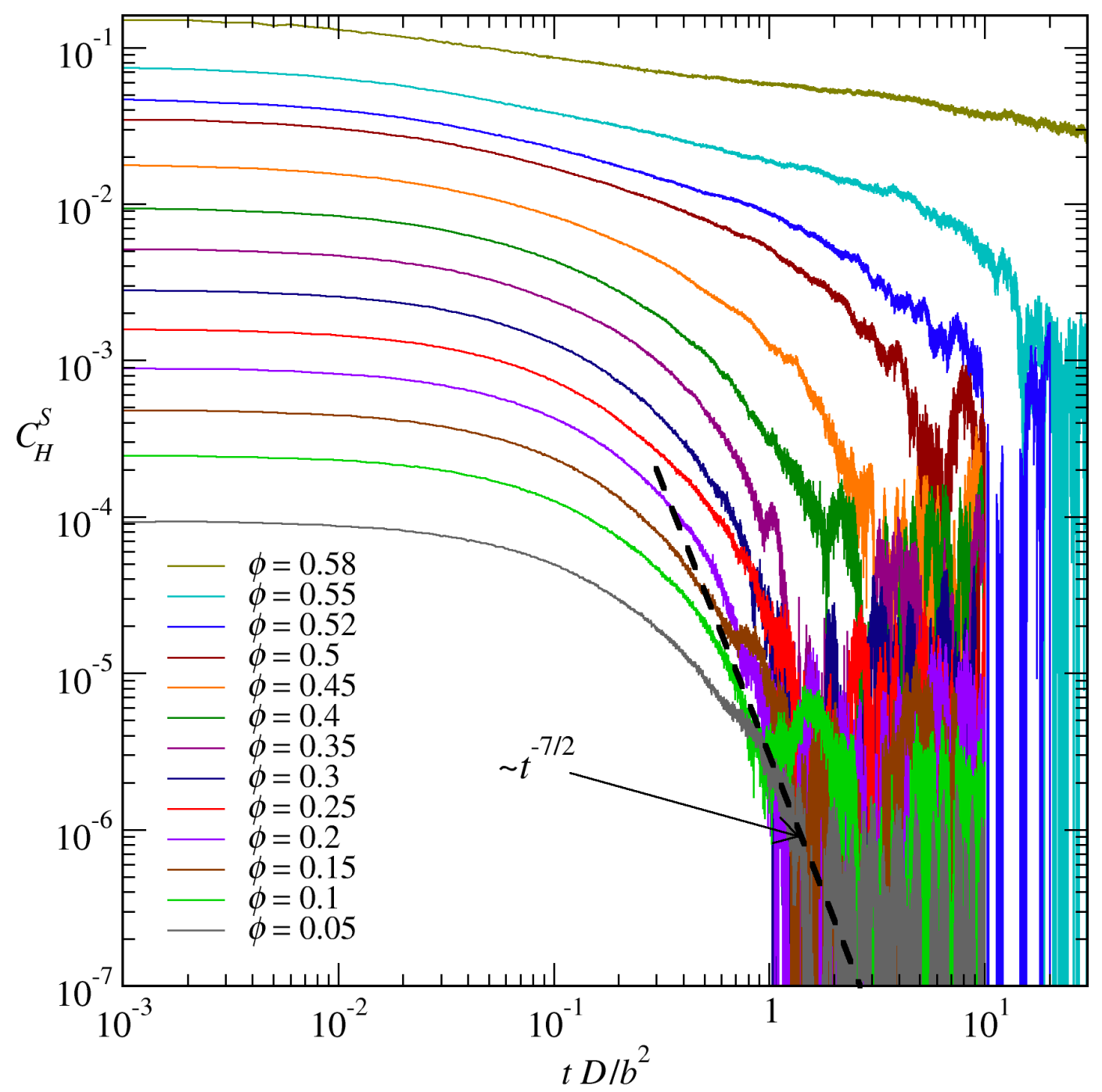

Figure 5.22: Shear stress autocorrelation function from Accelerated Stokesian Dynamics - near field simulations with $N=1000$ particles as a function of the diffusive time. For $\phi>0.55$ the system is in a glassy state so the shear stress autocorrelation decays much more slowly. 

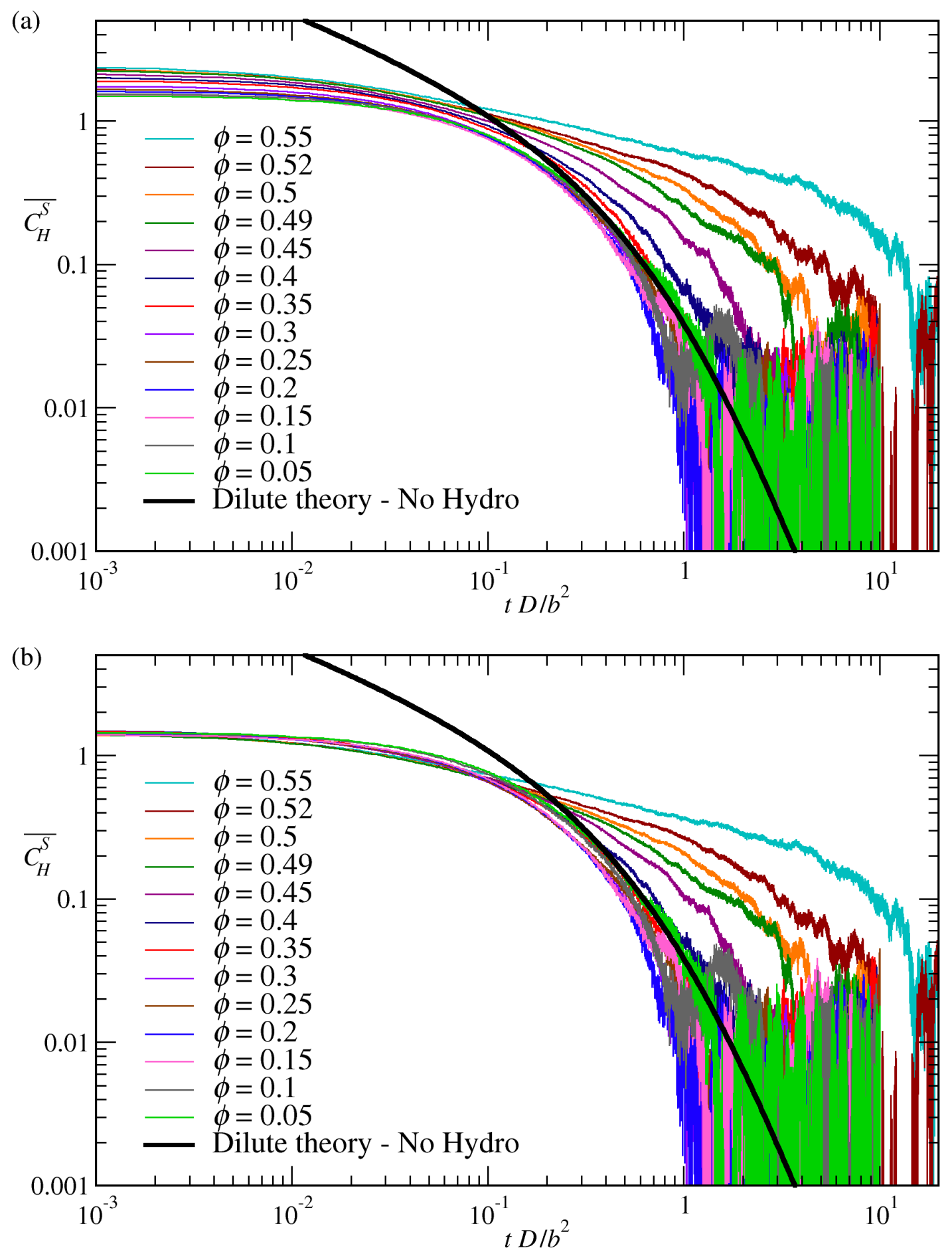

Figure 5.23: The scaled shear stress autocorrelation function from ASDB-nf simulations with $N=$ 1000 particles as a function of the diffusive time. The scaling in (a) is with the short-time selfdiffusivity $\overline{C_{H}^{S}}=C_{H}^{S} N D_{0}^{s}(\phi) /\left(\phi g_{0}(2 ; \phi)\right)$, and in (b) with the high-frequency shear viscosity $\overline{C_{H}^{S}}=$ $C_{H}^{S} N /\left(\phi g_{0}(2 ; \phi) \eta_{\infty}^{\prime}(\phi)\right)$. The analytical curve for no hydrodynamic interactions is also shown and has the same long-time behavior as the simulation data. 

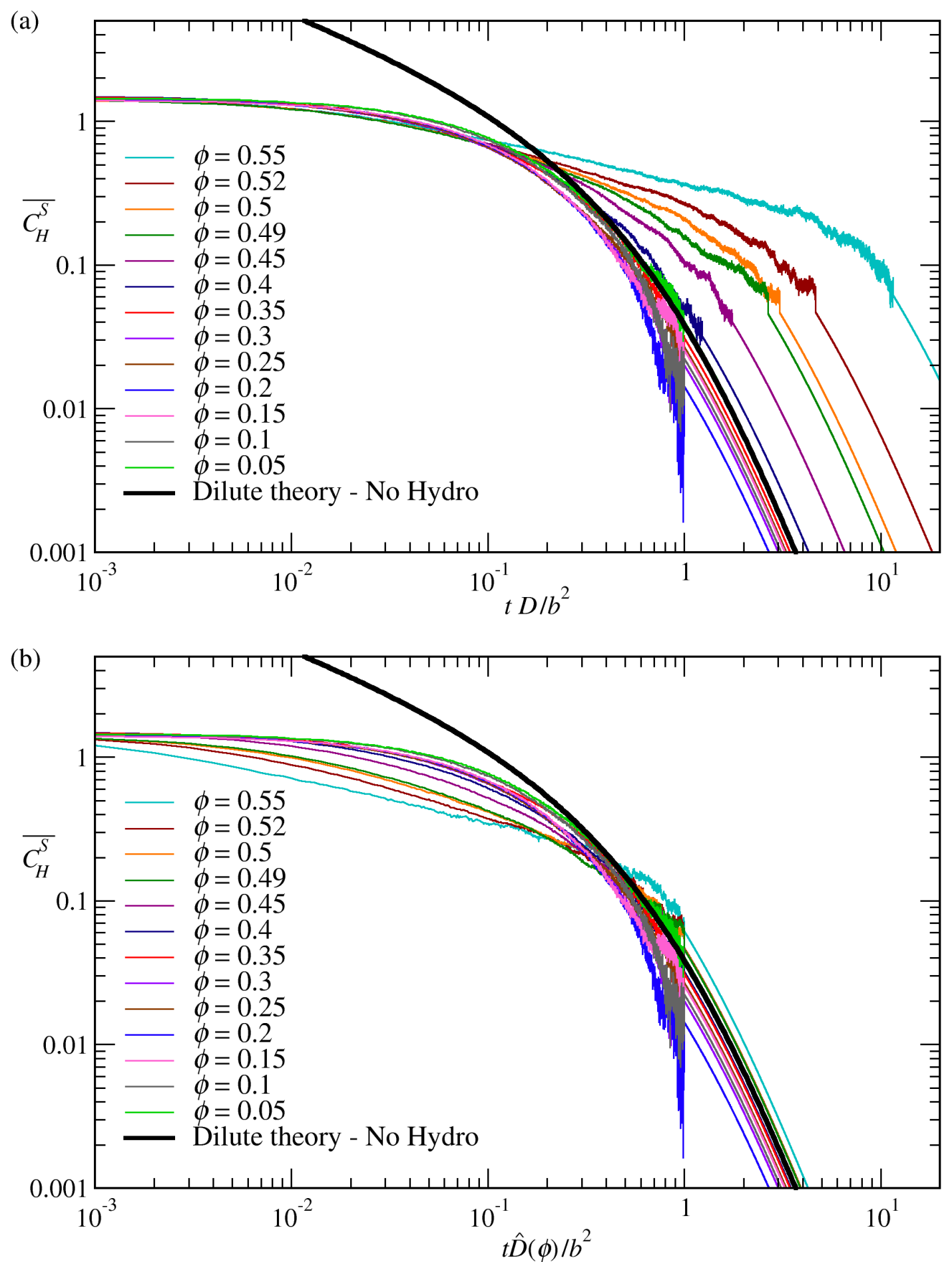

Figure 5.24: The scaled shear stress autocorrelation function from ASDB-nf simulations with $N=1000$ as a function of (a) the diffusive time, and (b) the diffusive time scaled with the stress relaxation time scale. The shear stress autocorrelation is scaled with the high-frequency shear vis$\operatorname{cosity} \overline{C_{H}^{S}}=C_{H}^{S} N /\left(\phi g_{0}(2 ; \phi) \eta_{\infty}^{\prime}(\phi)\right)$. The long-time tails are fitted with the analytical curve with no hydrodynamic interactions. 


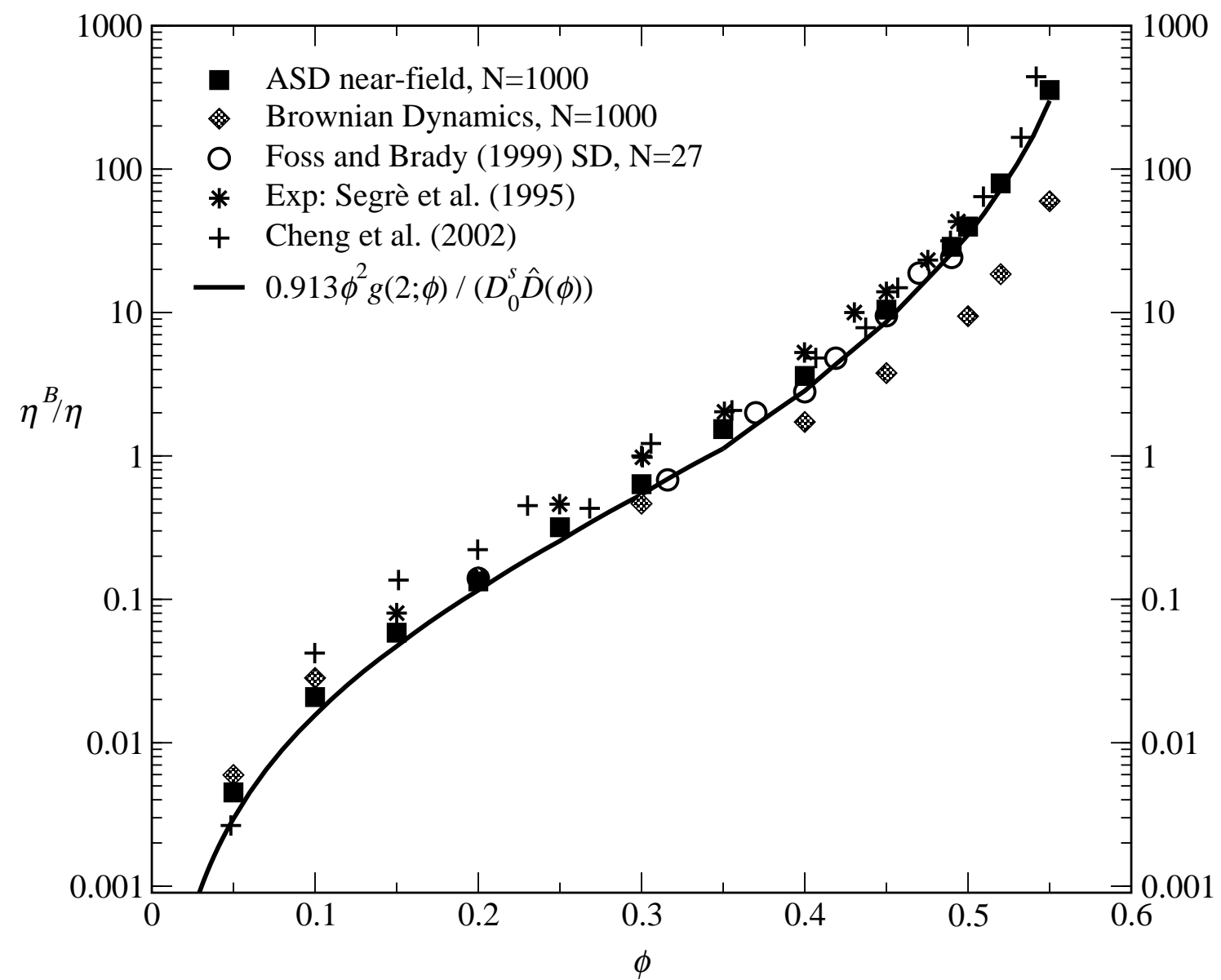

Figure 5.25: The Green-Kubo shear viscosity $\overline{\eta^{B}}$ as a function of volume fraction from equilibrium ASDB-nf simulations (solid squares) with $N=1000$, Brownian Dynamics simulations (shaded diamonds) with $N=1000$, and the scaled dilute theory (solid line). Also shown are the simulation results of Foss and Brady [1999] (empty circles), experimental results of Segrè et al. [1995], and experimental results of Cheng et al. [2002]. 


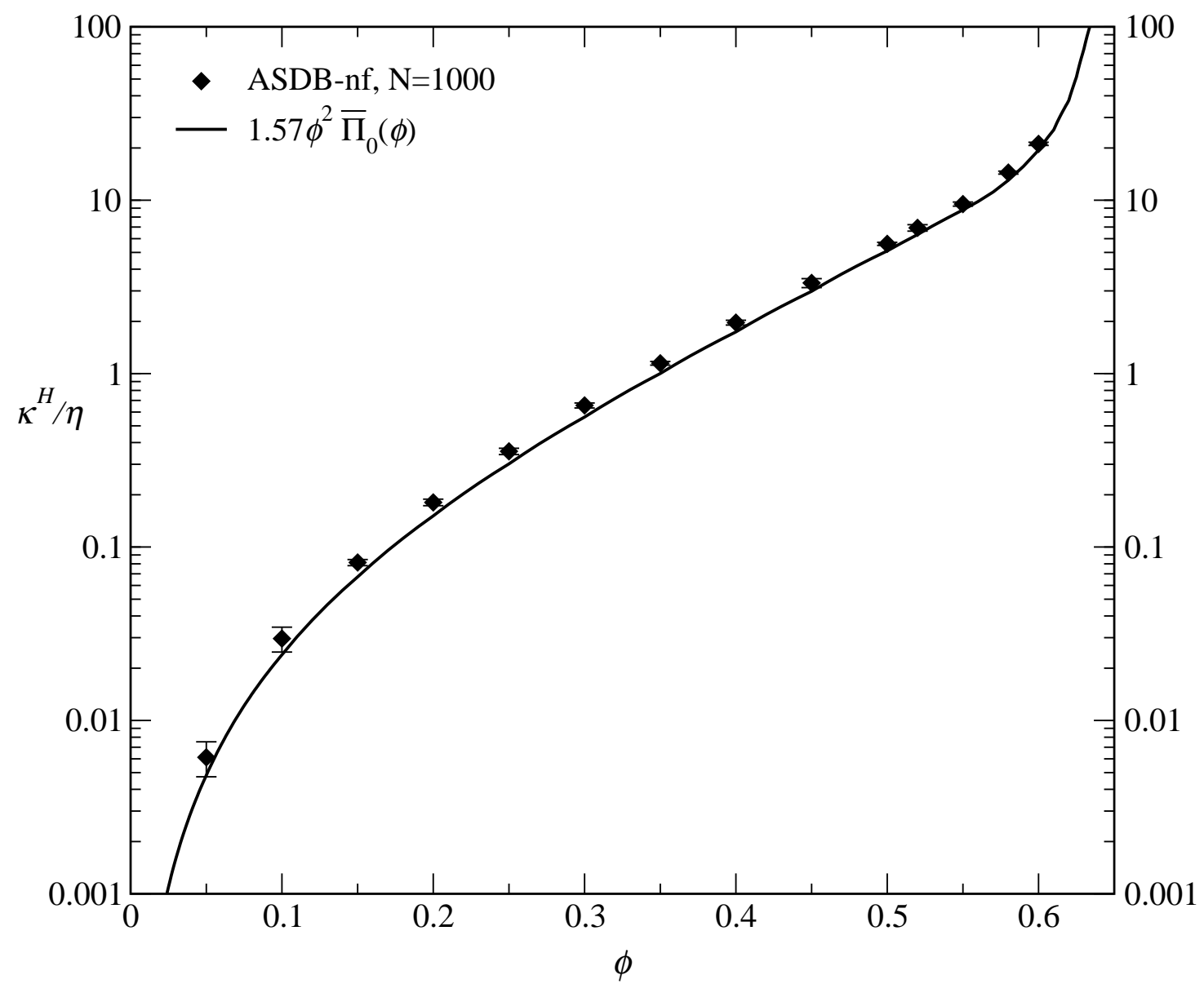

Figure 5.26: The high-frequency bulk viscosity due to interactions between particles as a function of volume fraction from equilibrium ASDB-nf simulations with $N=1000$, and the scaled dilute theory (solid line). 


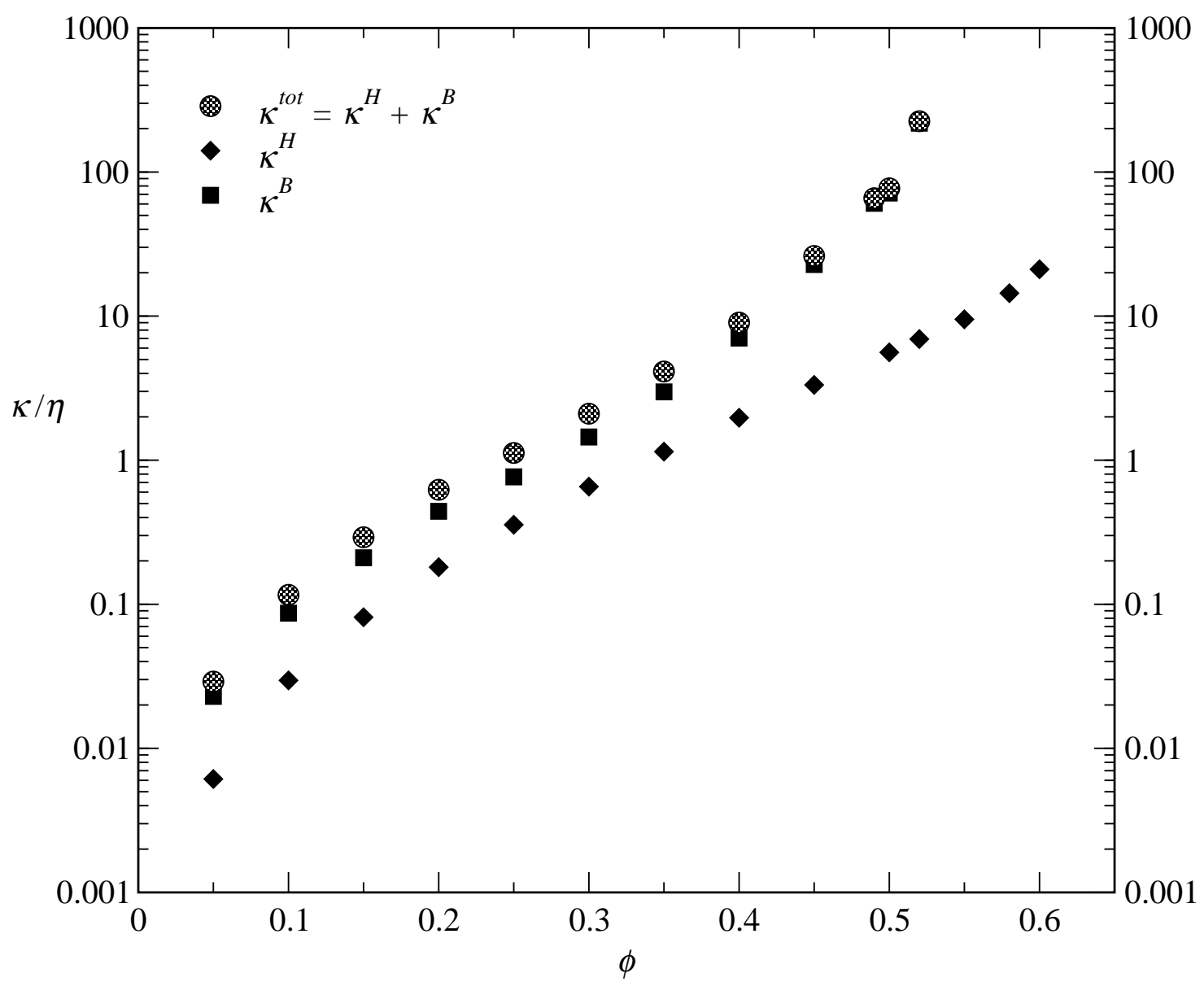

Figure 5.27: The total contribution to the bulk viscosity due to interactions between particles as a function of volume fraction from equilibrium ASDB-nf simulations with $N=1000$. Shown here is the direct hydrodynamic contribution (solid squares), the Green-Kubo Brownian contribution (solid squares), and their sum (shaded circles). 


\begin{tabular}{|cccccc|}
\hline$\phi$ & $N$ & $\Delta t\left(2 D / b^{2}\right)$ & Time steps & $\bar{\Pi}_{0}$ & Error $( \pm)$ \\
\hline \hline 0.05 & 1000 & $2 \times 10^{-4}$ & 20000000 & 1.2236 & 0.0018 \\
0.10 & 1000 & $2 \times 10^{-4}$ & 20000000 & 1.5118 & 0.0014 \\
0.15 & 1000 & $2 \times 10^{-4}$ & 20000000 & 1.8863 & 0.0016 \\
0.20 & 1000 & $2 \times 10^{-4}$ & 20000000 & 2.3766 & 0.0011 \\
0.25 & 1000 & $2 \times 10^{-4}$ & 20000000 & 3.0250 & 0.0006 \\
0.30 & 1000 & $2 \times 10^{-4}$ & 20000000 & 3.8930 & 0.0014 \\
0.35 & 1000 & $2 \times 10^{-4}$ & 20000000 & 5.0739 & 0.0028 \\
0.40 & 1000 & $2 \times 10^{-4}$ & 20000000 & 6.7053 & 0.0054 \\
0.45 & 1000 & $2 \times 10^{-4}$ & 20000000 & 9.0153 & 0.0088 \\
0.50 & 1000 & $2 \times 10^{-4}$ & 15000000 & 12.3759 & 0.0070 \\
0.52 & 1000 & $2 \times 10^{-4}$ & 15000000 & 14.1565 & 0.0128 \\
0.55 & 1000 & $2 \times 10^{-4}$ & 5000000 & 17.5007 & 0.0495 \\
0.58 & 1000 & $2 \times 10^{-4}$ & 5000000 & 21.5468 & 0.2059 \\
0.60 & 1000 & $2 \times 10^{-4}$ & 5000000 & 28.6155 & 0.7070 \\
\hline
\end{tabular}

Table 5.1: Equilibrium Osmotic Pressure from Brownian Dynamics simulations. 
153

\begin{tabular}{|cccccc|}
\hline$\phi$ & $N$ & $\Delta t\left(2 D / b^{2}\right)$ & Time steps & $\bar{\Pi}_{0}$ & Error $( \pm)$ \\
\hline \hline 0.05 & 1000 & $2 \times 10^{-3}$ & 2000000 & 1.2257 & 0.0006 \\
0.10 & 1000 & $2 \times 10^{-3}$ & 2000000 & 1.5176 & 0.0010 \\
0.15 & 1000 & $2 \times 10^{-3}$ & 2000000 & 1.8992 & 0.0009 \\
0.20 & 1000 & $2 \times 10^{-3}$ & 2000000 & 2.4038 & 0.0027 \\
0.25 & 1000 & $2 \times 10^{-3}$ & 2000000 & 3.0757 & 0.0018 \\
0.30 & 1000 & $2 \times 10^{-3}$ & 2000000 & 3.9822 & 0.0016 \\
0.35 & 1000 & $2 \times 10^{-3}$ & 2000000 & 5.2237 & 0.0024 \\
0.40 & 1000 & $2 \times 10^{-3}$ & 2000000 & 6.9667 & 0.0063 \\
0.45 & 1000 & $2 \times 10^{-3}$ & 2000000 & 9.4453 & 0.0071 \\
0.50 & 1000 & $2 \times 10^{-3}$ & 1500000 & 13.1005 & 0.0302 \\
0.52 & 1000 & $2 \times 10^{-3}$ & 1500000 & 15.0720 & 0.0478 \\
0.55 & 1000 & $2 \times 10^{-3}$ & 900000 & 18.5913 & 0.2460 \\
0.58 & 1000 & $2 \times 10^{-3}$ & 900000 & 25.6836 & 0.1754 \\
0.60 & 1000 & $2 \times 10^{-3}$ & 900000 & 36.6900 & 0.4836 \\
\hline
\end{tabular}

Table 5.2: Equilibrium Osmotic Pressure from ASDB-nf simulations.

\begin{tabular}{|c|c|c|c|}
\hline$\phi$ & $D_{\infty}^{s}$ & $\tau_{\eta}$ & $\tau_{\eta} D_{\infty}^{s}$ \\
\hline \hline 0.0500 & 0.9228 & 0.1835 & 0.1693 \\
\hline 0.1000 & 0.8437 & 0.1991 & 0.1680 \\
\hline 0.2000 & 0.6803 & 0.1647 & 0.1121 \\
\hline 0.3000 & 0.5096 & 0.1405 & 0.0716 \\
\hline 0.4000 & 0.3283 & 0.2009 & 0.0659 \\
\hline 0.4500 & 0.2304 & 0.3202 & 0.0738 \\
\hline 0.5000 & 0.1320 & 0.4235 & 0.0559 \\
\hline 0.5200 & 0.0981 & 0.8135 & 0.0798 \\
\hline 0.5500 & 0.0473 & 2.1509 & 0.1017 \\
\hline
\end{tabular}

Table 5.3: Relaxation time-scale for the shear stress autocorrelation function from Brownian Dynamics simulations with $N=1000$ particles. 


\begin{tabular}{|cccc|}
\hline$\phi$ & $\tau_{\kappa}(0.7)$ & $\tau_{\kappa}(200)$ & $D_{\infty}^{s}(\phi) \tau_{\kappa}(200)$ \\
\hline \hline 0.05 & 0.1671 & 10.6762 & 9.8515 \\
0.10 & 0.1335 & 6.1263 & 5.1690 \\
0.15 & 0.0972 & 4.4573 & 3.4006 \\
0.20 & 0.0883 & 4.5219 & 3.0765 \\
0.25 & 0.0688 & 3.9160 & 2.3071 \\
0.30 & 0.0621 & 3.7567 & 1.9144 \\
0.35 & 0.0446 & 3.3878 & 1.3681 \\
0.40 & 0.0590 & 3.8578 & 1.2665 \\
0.45 & 0.1231 & 5.5633 & 1.2816 \\
0.50 & 0.1964 & 9.9689 & 1.3154 \\
0.52 & 0.2249 & 10.3204 & 1.0123 \\
\hline
\end{tabular}

Table 5.4: Relaxation time-scale for pressure autocorrelation function from Brownian Dynamics simulations with $N=1000$ particles. 


\begin{tabular}{|cccc|}
\hline$\phi$ & $\tau_{\kappa}(1)$ & $\tau_{\kappa}(200)$ & $D_{\infty}^{s}(\phi) \tau_{\kappa}(200)$ \\
\hline \hline 0.05 & 0.2912 & 10.4930 & 9.6825 \\
0.10 & 0.2647 & 9.5283 & 8.0394 \\
0.15 & 0.2415 & 8.6424 & 6.5936 \\
0.20 & 0.2379 & 8.6723 & 5.9002 \\
0.25 & 0.2163 & 7.6328 & 4.4969 \\
0.30 & 0.2162 & 7.5952 & 3.8705 \\
0.35 & 0.2056 & 7.6431 & 3.0866 \\
0.40 & 0.2299 & 8.3879 & 2.7536 \\
0.45 & 0.3085 & 10.3517 & 2.3847 \\
0.50 & 0.3476 & 14.2023 & 1.8740 \\
0.52 & 0.3983 & 19.0036 & 1.8641 \\
0.55 & 0.4537 & 34.0876 & 1.6123 \\
0.58 & 0.4792 & 41.8469 & 0.8369 \\
\hline
\end{tabular}

Table 5.5: Relaxation time-scale for pressure autocorrelation function from Accelerated Stokesian Dynamics - near field simulations with $N=1000$ particles. 


\begin{tabular}{|cccccc|}
\hline$\phi$ & $N$ & $\Delta t\left(2 D / b^{2}\right)$ & Time steps & $\kappa^{B} / \eta$ & Error $( \pm)$ \\
\hline \hline 0.05 & 1000 & $2 \times 10^{-4}$ & 20000000 & 0.0268 & 0.0010 \\
0.10 & 1000 & $2 \times 10^{-4}$ & 20000000 & 0.0953 & 0.0023 \\
0.15 & 1000 & $2 \times 10^{-4}$ & 20000000 & 0.1858 & 0.0041 \\
0.20 & 1000 & $2 \times 10^{-4}$ & 20000000 & 0.3688 & 0.0056 \\
0.25 & 1000 & $2 \times 10^{-4}$ & 20000000 & 0.6032 & 0.0099 \\
0.30 & 1000 & $2 \times 10^{-4}$ & 20000000 & 1.0675 & 0.0146 \\
0.35 & 1000 & $2 \times 10^{-4}$ & 20000000 & 1.7615 & 0.0244 \\
0.40 & 1000 & $2 \times 10^{-4}$ & 20000000 & 3.4603 & 0.0378 \\
0.45 & 1000 & $2 \times 10^{-4}$ & 20000000 & 8.3705 & 0.0544 \\
0.50 & 1000 & $2 \times 10^{-4}$ & 15000000 & 30.8720 & 0.0875 \\
0.52 & 1000 & $2 \times 10^{-4}$ & 15000000 & 55.3136 & 0.1195 \\
\hline
\end{tabular}

Table 5.6: Equilibrium (Green-Kubo) bulk viscosity for hard spheres from Brownian Dynamics simulations. 


\begin{tabular}{|cccccc|}
\hline$\phi$ & $N$ & $\Delta t\left(2 D / b^{2}\right)$ & Time steps & $\kappa^{B} / \eta$ & Error $( \pm)$ \\
\hline \hline 0.05 & 1000 & $2 \times 10^{-3}$ & 2000000 & 0.0229 & 0.0003 \\
0.10 & 1000 & $2 \times 10^{-3}$ & 2000000 & 0.0867 & 0.0008 \\
0.15 & 1000 & $2 \times 10^{-3}$ & 2000000 & 0.2102 & 0.0016 \\
0.20 & 1000 & $2 \times 10^{-3}$ & 2000000 & 0.4420 & 0.0023 \\
0.25 & 1000 & $2 \times 10^{-3}$ & 2000000 & 0.7661 & 0.0041 \\
0.30 & 1000 & $2 \times 10^{-3}$ & 2000000 & 1.4483 & 0.0063 \\
0.35 & 1000 & $2 \times 10^{-3}$ & 2000000 & 2.9776 & 0.0137 \\
0.40 & 1000 & $2 \times 10^{-3}$ & 2000000 & 7.0446 & 0.0285 \\
0.45 & 1000 & $2 \times 10^{-3}$ & 2000000 & 22.8100 & 0.0384 \\
0.49 & 1000 & $2 \times 10^{-3}$ & 1500000 & 60.6845 & 0.1215 \\
0.50 & 1000 & $2 \times 10^{-3}$ & 1500000 & 71.4577 & 0.1758 \\
0.52 & 1000 & $2 \times 10^{-3}$ & 1500000 & 218.2948 & 0.5120 \\
\hline
\end{tabular}

Table 5.7: Equilibrium (Green-Kubo) bulk viscosity for hard spheres with hydrodynamic interactions from ASDB-nf simulations. 


\begin{tabular}{|cccccc|}
\hline$\phi$ & $N$ & $\Delta t\left(2 D / b^{2}\right)$ & Time steps & $\kappa^{B} / \eta$ & Error $( \pm)$ \\
\hline \hline 0.05 & 27 & $1 \times 10^{-3}$ & 3000000 & 0.0240 & 0.0163 \\
0.10 & 27 & $1 \times 10^{-3}$ & 3000000 & 0.0827 & 0.0521 \\
0.15 & 27 & $1 \times 10^{-3}$ & 3000000 & 0.1964 & 0.0567 \\
0.20 & 27 & $1 \times 10^{-3}$ & 3000000 & 0.3114 & 0.1152 \\
0.25 & 27 & $1 \times 10^{-3}$ & 3000000 & 0.7329 & 0.1520 \\
0.30 & 27 & $1 \times 10^{-3}$ & 4000000 & 1.4751 & 0.2917 \\
0.35 & 27 & $1 \times 10^{-3}$ & 4000000 & 2.4339 & 0.5630 \\
0.40 & 27 & $1 \times 10^{-3}$ & 4000000 & 7.2241 & 0.7421 \\
0.45 & 27 & $1 \times 10^{-3}$ & 4000000 & 27.4283 & 2.2675 \\
0.47 & 27 & $5 \times 10^{-4}$ & 8000000 & 26.1497 & 2.0049 \\
0.49 & 27 & $5 \times 10^{-4}$ & 8000000 & 16.9814 & 1.7155 \\
0.50 & 27 & $5 \times 10^{-4}$ & 8000000 & 33.0086 & 3.2338 \\
\hline
\end{tabular}

Table 5.8: Equilibrium (Green-Kubo) bulk viscosity for hard spheres with hydrodynamic interactions from conventional Stokesian Dynamics simulations. 


\begin{tabular}{|cccccc|}
\hline$\phi$ & $N$ & $\Delta t\left(2 D / b^{2}\right)$ & Time steps & $K_{\infty}^{\prime} b^{3} / k T$ & Error $( \pm)$ \\
\hline \hline 0.05 & 1000 & $2 \times 10^{-3}$ & 2000000 & 0.0026 & $1.6663 \times 10^{-6}$ \\
0.10 & 1000 & $2 \times 10^{-3}$ & 2000000 & 0.0125 & $3.9464 \times 10^{-6}$ \\
0.15 & 1000 & $2 \times 10^{-3}$ & 2000000 & 0.0357 & $8.0360 \times 10^{-6}$ \\
0.20 & 1000 & $2 \times 10^{-3}$ & 2000000 & 0.0815 & $1.1640 \times 10^{-5}$ \\
0.25 & 1000 & $2 \times 10^{-3}$ & 2000000 & 0.1709 & $2.0572 \times 10^{-5}$ \\
0.30 & 1000 & $2 \times 10^{-3}$ & 2000000 & 0.3390 & $3.1711 \times 10^{-5}$ \\
0.35 & 1000 & $2 \times 10^{-3}$ & 2000000 & 0.6880 & $6.8662 \times 10^{-5}$ \\
0.40 & 1000 & $2 \times 10^{-3}$ & 2000000 & 1.4302 & $1.4235 \times 10^{-4}$ \\
0.45 & 1000 & $2 \times 10^{-3}$ & 2000000 & 3.0960 & $1.9187 \times 10^{-4}$ \\
0.49 & 1000 & $2 \times 10^{-3}$ & 1500000 & 5.5672 & $6.0754 \times 10^{-4}$ \\
0.50 & 1000 & $2 \times 10^{-3}$ & 1500000 & 6.2335 & $8.7901 \times 10^{-4}$ \\
0.52 & 1000 & $2 \times 10^{-3}$ & 1500000 & 9.3763 & $2.5599 \times 10^{-3}$ \\
\hline
\end{tabular}

Table 5.9: High-frequency bulk modulus for hard spheres with hydrodynamic interactions from ASDB-nf simulations. 


\begin{tabular}{|cccccc|}
\hline$\phi$ & $N$ & $\Delta t\left(2 D / b^{2}\right)$ & Time steps & $K_{\infty}^{\prime} b^{3} / k T$ & Error( \pm$)$ \\
\hline \hline 0.05 & 27 & $1 \times 10^{-3}$ & 3000000 & 0.0031 & 0.0001 \\
0.10 & 27 & $1 \times 10^{-3}$ & 3000000 & 0.0147 & 0.0003 \\
0.15 & 27 & $1 \times 10^{-3}$ & 3000000 & 0.0421 & 0.0003 \\
0.20 & 27 & $1 \times 10^{-3}$ & 3000000 & 0.0988 & 0.0006 \\
0.25 & 27 & $1 \times 10^{-3}$ & 3000000 & 0.2086 & 0.0008 \\
0.30 & 27 & $1 \times 10^{-3}$ & 4000000 & 0.4224 & 0.0015 \\
0.35 & 27 & $1 \times 10^{-3}$ & 4000000 & 0.8543 & 0.0028 \\
0.40 & 27 & $1 \times 10^{-3}$ & 4000000 & 1.7259 & 0.0037 \\
0.45 & 27 & $1 \times 10^{-3}$ & 4000000 & 3.5274 & 0.0113 \\
0.47 & 27 & $5 \times 10^{-4}$ & 8000000 & 4.3977 & 0.0100 \\
0.49 & 27 & $5 \times 10^{-4}$ & 8000000 & 5.5707 & 0.0086 \\
0.50 & 27 & $5 \times 10^{-4}$ & 8000000 & 6.5465 & 0.0162 \\
\hline
\end{tabular}

Table 5.10: High-frequency bulk modulus for hard spheres with hydrodynamic interactions from conventional Stokesian Dynamics simulations. 


\begin{tabular}{|ccccc|}
\hline$\phi$ & $N$ & Realizations & $\kappa_{\infty}^{\prime} / \eta$ & Error( \pm ) \\
\hline \hline 0.05 & 1000 & 400 & 0.0061 & 0.0014 \\
0.10 & 1000 & 400 & 0.0296 & 0.0048 \\
0.15 & 1000 & 400 & 0.0814 & 0.0033 \\
0.20 & 1000 & 400 & 0.1808 & 0.0078 \\
0.25 & 1000 & 400 & 0.3561 & 0.0147 \\
0.30 & 1000 & 400 & 0.6551 & 0.0216 \\
0.35 & 1000 & 400 & 1.1466 & 0.0281 \\
0.40 & 1000 & 400 & 1.9690 & 0.0622 \\
0.45 & 1000 & 400 & 3.3316 & 0.1974 \\
0.50 & 1000 & 300 & 5.6014 & 0.1108 \\
0.52 & 1000 & 300 & 6.9303 & 0.2920 \\
0.55 & 1000 & 300 & 9.4979 & 0.2594 \\
0.58 & 1000 & 200 & 14.4265 & 0.2901 \\
0.60 & 1000 & 200 & 21.1343 & 0.4494 \\
\hline
\end{tabular}

Table 5.11: High-frequency bulk viscosity for hard spheres with hydrodynamic interactions from ASDB-nf simulations. 


\begin{tabular}{|cccccc|}
\hline$\phi$ & $N$ & $\Delta t\left(2 D / b^{2}\right)$ & Time steps & $\eta^{B} / \eta$ & Error( \pm$)$ \\
\hline \hline 0.05 & 1000 & $2 \times 10^{-4}$ & 20000000 & 0.0060 & 0.0002 \\
0.10 & 1000 & $2 \times 10^{-4}$ & 20000000 & 0.0283 & 0.0005 \\
0.20 & 1000 & $2 \times 10^{-4}$ & 20000000 & 0.1422 & 0.0012 \\
0.30 & 1000 & $2 \times 10^{-4}$ & 20000000 & 0.4648 & 0.0019 \\
0.40 & 1000 & $2 \times 10^{-4}$ & 20000000 & 1.7235 & 0.0044 \\
0.45 & 1000 & $2 \times 10^{-4}$ & 20000000 & 3.7821 & 0.0071 \\
0.50 & 1000 & $2 \times 10^{-4}$ & 15000000 & 9.4250 & 0.0116 \\
0.52 & 1000 & $2 \times 10^{-4}$ & 15000000 & 18.5061 & 0.0161 \\
0.55 & 1000 & $2 \times 10^{-4}$ & 15000000 & 59.6699 & 0.0288 \\
\hline
\end{tabular}

Table 5.12: Equilibrium (Green-Kubo) shear viscosity for hard spheres from Brownian Dynamics simulations.

\begin{tabular}{|cccccc|}
\hline$\phi$ & $N$ & $\Delta t\left(2 D / b^{2}\right)$ & Time steps & $\eta^{B} / \eta$ & Error $( \pm)$ \\
\hline \hline 0.05 & 1000 & $2 \times 10^{-3}$ & 2000000 & 0.0045 & 0.0001 \\
0.10 & 1000 & $2 \times 10^{-3}$ & 2000000 & 0.0208 & 0.0001 \\
0.15 & 1000 & $2 \times 10^{-3}$ & 2000000 & 0.0586 & 0.0003 \\
0.20 & 1000 & $2 \times 10^{-3}$ & 2000000 & 0.1333 & 0.0004 \\
0.25 & 1000 & $2 \times 10^{-3}$ & 2000000 & 0.3182 & 0.0008 \\
0.30 & 1000 & $2 \times 10^{-3}$ & 2000000 & 0.6355 & 0.0014 \\
0.35 & 1000 & $2 \times 10^{-3}$ & 2000000 & 1.5390 & 0.0039 \\
0.40 & 1000 & $2 \times 10^{-3}$ & 2000000 & 3.6116 & 0.0058 \\
0.45 & 1000 & $2 \times 10^{-3}$ & 2000000 & 10.4687 & 0.0090 \\
0.49 & 1000 & $2 \times 10^{-3}$ & 1500000 & 28.7002 & 0.0488 \\
0.50 & 1000 & $2 \times 10^{-3}$ & 1500000 & 39.7402 & 0.0662 \\
0.52 & 1000 & $2 \times 10^{-3}$ & 1500000 & 79.5143 & 0.1975 \\
\hline
\end{tabular}

Table 5.13: Equilibrium (Green-Kubo) shear viscosity for hard spheres with hydrodynamic interactions from ASDB-nf simulations. 


\section{Chapter 6}

\section{Simulation of Compression Flows in Suspensions}

\subsection{Introduction}

Colloidal suspensions are known to exhibit non-Newtonian behavior, meaning that the rheological properties change with the applied rate of strain. Thinning of the effective suspension shear viscosity has been observed at low shear rates and thickening has been observed at high shear rates [Gadala-Maria and Acrivos 1980; Brady and Morris 1997; Sierou and Brady 2002]. The nondimensional number that defines the regime of flow is the Péclet number $(\mathrm{Pe})$, which gives the ratio of the applied forcing to the strength of Brownian motion. Shear thinning occurs as the contribution to the effective shear viscosity of the suspension due to Brownian motion decreases with increasing $P e$. In the large $\mathrm{Pe}$ limit particles are pushed into the compression axis and depleted in the elongation axis and Brownian motion is not strong enough to dissipate the disturbance. A boundary layer forms at contact because of the rigid particle surfaces, inside which Brownian motion balances convection. Lubrication forces are very strong in this region and cause the shear viscosity to increase, and this is called shear thickening. In compression flow of suspensions we expect the same behavior, except there must be 'compression thinning' at small $P e$ as Brownian motion becomes weaker than convection, and 'compression thickening' at high $\mathrm{Pe}$ as the convective flow pushes the particles together leading to formation of an isotropic boundary layer in which there is accumulation of particles. Dilute theory shows that the boundary layer thickness in compression is of order $1 / P e$, while the accumulation in the boundary layer is order $P e$ in both the limiting cases of no hydrodynamic interactions and full hydrodynamics. The bulk viscosity therefore plateaus to a constant at large Péclet 
numbers. To study the high $P e$ response of concentrated suspensions we must resort to numerical simulation.

Simulating the expansion (or compression) of only the particle phase in a suspension would necessarily lead to spatial variations in the particle volume-fraction, which make it difficult to determine the bulk viscosity for a given volume-fraction. This problem can be circumvented by having the surrounding fluid expand or compress uniformly in space, leading to a homogeneous expansion or compression of the particle phase as well. The imposed flow causes the suspended particles to move apart uniformly in space, but they cannot expand with the fluid and instead create disturbance flows as the fluid has to move around the particles. This disturbance flow causes the stress on the particles to change, also changing the bulk stress in the suspension. The bulk viscosity of the suspension is then determined by computing the deviation in average stress in the material in a way analogous to that for the shear viscosity [Batchelor and Green 1972b; Brady and Bossis 1988], and relating it to the average rate of expansion. We expect the bulk viscosity calculated by this technique to be comparable to that for expansion of the particle phase only in an incompressible fluid, because the contribution to the isotropic stress in both cases is due to incompressible disturbance flows. In this chapter we describe the numerical simulations that were performed to determine the bulk viscosity of a suspension at all rates of compression for a wide range of volume fractions.

\subsection{Simulation procedure}

All the compression simulations were started with a random configuration at a small volume fraction of $\phi=0.01$, generated by simply placing the particles at randomly generated positions inside the simulation cell and then resolving any particle overlaps. At this small volume fraction there are few particle overlaps so that the random nature of the configuration is not affected by moving the particles to resolve overlaps. At each time step compression was achieved by changing the dimensions of the simulation cell in the $x, y$ and $z$ coordinates by $L_{i} P e \Delta t$, where $P e$ is the Péclet number, $\Delta t$ is the nondimensional time step and $L_{i}$ is the size of the simulation cell along the $i^{\text {th }}$ coordinate nondimensionalized with the particle radius $b$. The Péclet number is defined as the bulk rate of 
compression $\langle e\rangle$ nondimensionalized with the diffusive time-scale of the particles $b^{2} / 2 D$ as

$$
P e=\frac{\frac{1}{3}\langle e\rangle b^{2}}{2 D}
$$

where $D=k T / 6 \pi \eta b$ is the Stokes-Einstein-Sutherland diffusivity of the particles. Time was nondimensionalized with the relative diffusive time scale of the particles $b^{2} / 2 D$. For $|P e|>1$ the time was additionally scaled with the Péclet number by replacing $\Delta t$ with $\Delta t /|P e|$ so that changes in the simulation cell size and particle positions are not too large for a single time-step. At the same time an affine displacement of $r_{i} P e \Delta t$ was applied to each particle where $r_{i}$ is the position vector of the particle scaled with $b$, and displacements due to hydrodynamic interactions and Brownian motion were also added to the particle positions. The Péclet number is positive for expansion flow and negative for compression. Periodic boundary conditions were enforced to ensure that all particles remain within the simulation cell and collisions between particles were resolved to remove any particle overlaps. In the case of Brownian Dynamics particle collisions were used to determine the particle-phase stress, but in SD and ASD simulations the collisions are only an artifact of the finite time step used because lubrication forces would prevent particle surfaces from ever touching, and therefore collisions do not contribute to the stress. The cell volume changes by a factor of $(1+P e \Delta t)^{3}$ and therefore the change in volume fraction is $\Delta \phi=\phi /(1+P e \Delta t)^{3}$ at each time step. Since $\Delta \phi$ is proportional to the volume fraction $\phi$ itself, starting with a small $\phi$ causes the volume fraction to change very slowly in the beginning of the simulation, thus allowing sufficient time for diffusive and convective motion of the particles to balance each other to give a steady particle microstructure before it reaches volume fractions of our interest, $\phi \geq 0.1$.

Although the number density of particles changes continuously with time in the simulations, the distribution of particles relative to the average number density reaches a non-equilibrium steady state in compression flow. The imposed flow acts to push the particles closer to each other thereby driving the microstructure out of equilibrium, while Brownian motion drives diffusion against the concentration gradients to restore equilibrium, and the competition between the two establishes the steady microstructure. Starting the simulation with a small volume fraction allows the microstructure to achieve an almost steady state which we want to study before it reaches higher concentrations. The constantly changing number density however, presents the problem of reliably collecting 
statistically good data for a given volume fraction of particles. One way in which we overcome this hurdle is by performing a large number of simulations (200) for each Péclet number with the same simulation parameters but starting with different initial configurations. The data for the desired volume fractions is then extracted from each run and averaged to get an ensemble average. Additionally the data in each simulation run is averaged over several adjacent time-steps before and after the time step where the volume fraction of interest is reached, such that the data between $\phi-0.01$ and $\phi+0.01$ is averaged for each $\phi$. The change in the particle microstructure over short times would be minimal and averaging over the time interval helps to reduce Brownian noise. As long as the averaging is done over an equal number of time steps before and after the time of interest, no additional error is introduced due to the changing concentration. Thus averaging over several time steps in each run serves to reduce fluctuations due to Brownian motion of the particles and averaging over all the runs reduces the error due to variations in the microstructure. For computing the pair-distribution function of particles, averaging was done only across all the simulation runs because changes in the microstructure over short times are negligible.

\subsection{Microstructure in compression}

As the compression flow proceeds, particles are pushed towards each other by the imposed forcing, thus driving the system out of equilibrium with particles being closer to each other on average than they would be at equilibrium. This shows up as an isotropic accumulation of particles at contact $(r=2)$ in the pair-distribution function $g(r)$, which is a function of the scalar particle separation only because of the isotropic forcing. Brownian motion of the particles causes them to diffuse against the concentration gradient that is built up near contact and makes $g(r)$ decay with increasing $r$. For small rates of compression the perturbation to the microstructure decays as $1 / r$. At higher compression rates a boundary layer of $O\left(|P e|^{-1}\right)$ forms near contact in which Brownian diffusion balances the compression flow and the microstructural perturbation decays to zero inside the boundary layer. The magnitude of the microstructural perturbation inside the boundary layer is $O(|\mathrm{Pe}|)$ from dilute theory analysis. Figure 6.1 shows a planar cross-section of the three-dimensional pair distribution function computed from Brownian Dynamics compression simulations with 1000 particles. The figure illustrates the isotropic nature of the forcing in compression and the stronger accumulation 
and faster decay of particle concentration near contact with increasing $P e$. At higher volume fractions the formation of an additional ring of higher concentration can be seen as the caging effect of particles becomes more prominent, but the behavior of $g(r)$ with varying $P e$ is still the same.

In all the simulations we start with an equilibrium particle configuration, so the microstructure must evolve as part of the simulation. The time it takes to reach a steady state would be determined by the diffusive time-scale of the particles and the distance over which they need to diffuse in order to balance the compression flow. The simplest estimate for the diffusive time is $b^{2} / 2 D_{\infty}^{s}(\phi)$, where $D_{\infty}^{s}$ is the long-time self-diffusivity which accounts for the hindrance encountered by a particle as it makes its way through the surrounding particle structure. In regions of accumulation of particles the time scale of diffusion would be even slower than that given by $D_{\infty}^{s}$. The long-time self-diffusivity is a decreasing function of particle concentration so we expect that the approach to steady state would be slower with increasing $\phi$. However it is possible that the gradients in the microstructure reach a steady state before reaching high values of $\phi$ so that only the particle density is increasing while $g(r)$ remains unchanged.

The imposed compression forcing is balanced by Brownian motion only in the boundary layer so particles need to diffuse over a distance of $O\left(|P e|^{-1}\right)$ for the microstructure to reach a steady state. Figure 6.2 and Figure 6.3 show the radial pair-distribution function from BD and ASD simulations respectively at $P e=-1$ and $P e=-10$ for $\phi=0.2$. The data from both simulations is almost identical except for the value at contact, where lubrication forces in ASD simulations reduce the particle mobility significantly, causing a stronger accumulation of particles. Clearly $g(r)$ decays over a much longer distance at $P e=-1$ than at $P e=-10$, because particles need to diffuse over a shorter distance at larger values of $P e$ in order to balance the imposed forcing with Brownian diffusion. At the same time the volume fraction also changes at a faster rate for larger $\mathrm{Pe}$. Specifically the volume fraction as a function of time is given by

$$
\phi(\tau)=\phi(\tau) e^{-3 P e \tau}
$$

The change in volume fraction is exponential so that it changes very slowly at the start of our simulations when $\phi \sim 0.01$ but changes very quickly at higher volume fractions which are in the regime of interest. From the analysis in Appendix A in Chapter 1, the microstructural disturbance 
also approaches its long-time limit exponentially as $e^{-3 P e \tau}$ in the dilute limit for large Pe. Hence even in the dilute limit we would not expect the microstructure to be able to keep up with the change in volume fraction in the high $\mathrm{Pe}$ limit. Essentially the particles need to move a distance of $O(1)$ on average to be pushed into the boundary layer and this would not happen fast enough compared to the rate of change of the volume fraction, so the boundary layer formation remains incomplete. For small $P e$ the microstructure would change even more slowly (algebraically in the linear response limit) as shown in Chapter $3 \S 3.4$, and therefore would not achieve a steady state at any volume fraction.

In the above discussion we assumed that the initial configuration of particles is spatially homogeneous. While this is true in an average sense because we start from equilibrium configurations, any actual realization would have spatial inhomogeneities due to the random placement of particles. Thus there would be some regions where particles are already very close to each other and some other regions where particles are not close to any neighboring particle. At small Pe Brownian motion of the particles would cause these variations to dissipate and allow the particles to sample many random configurations, so that relative to the rate of the imposed compression flow the particle distribution is mostly homogeneous. In this case there is actual competition between the compression flow pushing the particles closer and Brownian motion dissipating the accumulation of particles. Particles would need to move an $O(1)$ distance in order to be pushed into the boundary layer. Therefore at small $P e$ of compression we don't expect the microstructural disturbance to reach a steady state.

At large $P e$ however, Brownian motion is negligible so groups of particle that were already close to each other are pushed even closer without any competition from Brownian diffusion, thereby creating clusters of particles scattered throughout the space where the boundary layer is formed very quickly. This is evident from Figure 6.4 which shows a histogram of the instantaneous hydrodynamic pressure moment of particles at rates of $P e=-1$ and $P e=-1000$ compiled over 200 runs of ASDB-nf compression simulations. At small $\mathrm{Pe}$ the pressure moment values are all very close to the average indicating that the particle distribution has been homogenized by Brownian motion. At high $\mathrm{Pe}$ however the distribution of pressure moments is more spread out and there is a significant number of particles that are quite a bit far from the mean, and these must be the particles that are close to other particles and thereby increase the overall stress due to lubrication forces. A look at 
Figure 6.5, which shows two such examples of particles with pressure moment greater than a cutoff value $\left(\left\langle S^{H}\right\rangle / P e>10\right)$ deduced from the histogram with $P e=-1000$, reveals that this is indeed the case. Clusters and chains of particles distributed throughout space are clearly visible in the figures and these are the regions of high particle pressures. There is a balance between the imposed flow and the boundary condition at contact that is set up in these regions, due to hard sphere collisions in the absence of hydrodynamics and lubrication forces near contact when hydrodynamics are important. The particle phase pressure in these regions would be much higher than in the rest of the suspension, resulting in a total stress that is much closer to the steady state value than at smaller $P e$. Therefore at large $P e$ even though the suspension on an average does not reach steady state there will be particle clusters throughout space depending on the initial configuration where the steady state boundary layer is formed and these clusters would grow in size as the compression of particles proceeds. The total particle-phase stress would be dominated by the stress in these clusters.

This does not undermine the results of the current topic because the main purpose of this study is to determine the bulk viscosity at high $P e$ via simulation. Whether the microstructure reaches the correct steady state or not may also depend on the number of particles used in the periodic simulation cell, which determines the cell size for a given $\phi$. If the size of the boundary layer is comparable to or greater than the cell length, the decay of $g(r)$ may not be captured correctly as the periodic boundary conditions impose an additional constraint on $g(r)$ at the cell boundaries.

\subsection{Brownian Dynamics}

In Brownian Dynamics simulations each hard sphere acts as a colloidal particle undergoing Brownian motion with the time scale specified by its bare diffusivity, but the effect of the disturbance flows due to the finite size of a particle on the other particles is neglected. Particles may only interact with each other via hard-sphere collisions when they come into contact. This approach allows us to isolate the effect of Brownian motion and interparticle forces on the microstructure from the influence of hydrodynamic interactions. The technique developed by Foss and Brady [2000] was employed to perform the Brownian Dynamics simulations. Each particle is given a random Brownian kick and an affine compression displacement at each time step, and particle collisions are resolved by moving the overlapped particles away from each other along the line joining their centers until they 
are touching. The interparticle force due to hard-sphere interactions for each particle is estimated as

$$
\boldsymbol{F}^{P}=6 \pi \eta b \frac{\Delta \boldsymbol{x}^{H S}}{\Delta t}
$$

where $\Delta \boldsymbol{x}^{H S}$ is the particle displacement from resolution of all the overlaps with other particles in time step $\Delta t$. The particle-phase contribution to the stress due to the collisions is simply

$$
\left\langle\Sigma^{P}\right\rangle=-n k T \boldsymbol{I}-n\left\langle\boldsymbol{x} \boldsymbol{F}^{P}\right\rangle
$$

where the angle brackets denote an average over all the particles, $\boldsymbol{x}$ is the particle position and $n$ is the number density of particles. The stress calculated with this technique contains the total stress due to Brownian motion as well as the contribution due to collisions resulting from the imposed forcing — compression flow in this case.

The effective bulk viscosity of the suspension can be written as

$$
\kappa_{e f f}=\left(\kappa+\frac{4}{3} \eta \phi\right) \frac{1}{1-\phi}+\kappa^{P}
$$

where the $\kappa^{P}$ term is the contribution from hard sphere particle collisions obtained directly from the trace of the stress as

$$
\kappa^{P}=\frac{n\left\langle S^{P}\right\rangle-n\left\langle S^{P}\right\rangle^{e q}}{3\langle e\rangle}
$$

Here $S^{P}$ denotes the trace of the corresponding stresslet (symmetric first moment of the force distribution integrated over the particle surface), i.e., $\left\langle S^{P}\right\rangle=\boldsymbol{I}:\left\langle\boldsymbol{x} \boldsymbol{F}^{P}\right\rangle$. The superscript $e q$ denotes the value at equilibrium, which corresponds to the equilibrium osmotic pressure of the particle phase. The first term in (6.5) gives the single particle correction to the bulk viscosity in a uniformly expanding fluid. The collisional contribution to the bulk viscosity nondimensionalized with the fluid shear viscosity can be written in terms of nondimensional quantities as

$$
\overline{\kappa^{P}}=\frac{3}{4} \phi \frac{\left(\bar{\Pi}-\bar{\Pi}_{0}\right)}{P e}
$$

where $\bar{\Pi}=\left\langle S^{P}\right\rangle / 3 k T$ is the nondimensional particle-phase pressure and $\bar{\Pi}_{0}$ is its value at equilib- 
rium. The hard-sphere equilibrium osmotic pressure is

$$
\bar{\Pi}_{0}=1+4 \phi g_{0}(2 ; \phi)
$$

where $g_{0}(2 ; \phi)$ is the equilibrium pair-distribution function at contact. An accurate value for $g_{0}(2 ; \phi)$ can be found from the well-known Carnahan-Starling (CS) equation of state [Carnahan and Starling 1969] for $\phi \leq 0.55$ :

$$
g_{0}(2 ; \phi)=\frac{1-\frac{1}{2} \phi}{(1-\phi)^{3}} .
$$

For higher volume fractions we have used the very precise values for $g_{0}(2 ; \phi)$ determined by Rintoul and Torquato [1996], which also match the CS equation of state at its limiting value of $\phi=0.5$.

Simulations were performed with $N=1000$ particles for several values of $P e$ ranging from 0.1 to 1000 , with 200 distinct runs for each value of $P e$. The simulations were started with a time step of $\Delta t=10^{-3}$ which was reduced to $2 \times 10^{-4}$ after reaching a volume fraction of $\phi=0.09$, thus saving computer time initially when the microstructure is changing very slowly and allowing for greater detail and better statistics in the regime of interest. The rheological data was recorded at each time step and particle positions were saved at volume fractions $\phi=0.1,0.15 \ldots 0.55$ with an interval of 0.05 . Values for the rheological properties were also evaluated for the same volume fractions from the simulation data. Particle configurations recorded for each value of $\phi$ studied were used to determine the pair distribution function averaged over all the runs for studying the microstructure.

\subsubsection{Results and scaling}

The contribution to the particle-phase pressure due to collisions between the particles, given by $\bar{\Pi}-1=\Pi / n k T-1$ is shown in Figure 6.6 as a function of the Péclet number for compression. The pressure asymptotes to its equilibrium value as $P e \rightarrow 0$ and for large values of $P e$ it scales as $|P e|$. In the large $P e$ limit the collisions driven by the imposed compression flow dominate the stress and therefore the pressure increases as the forcing becomes stronger. In the absence of hydrodynamic interactions the stress is completely determined by particle collisions as they come into contact and since the accumulation of particles at contact is $O(|P e|)$ the corresponding stress contribution is also $O(|P e|)$. Only the excess pressure due to the compression flow $\left(\Pi-\Pi_{0}\right)$ scales as 
$P e$, so that at low rates of compression the excess pressure is negligible compared to the equilibrium osmotic pressure. The osmotic pressure due to hard sphere collisions is known to scale as $\phi g_{0}(2 ; \phi)$ at equilibrium, which serves as the $\phi$ scaling for the average number of particle collisions [Brady 1993a]. Here $g_{0}(2 ; \phi)$ is the equilibrium pair distribution function at contact. Therefore we attempt to remove the $\phi$ scaling from the pressure by defining the normalized excess pressure as $\bar{\Pi}_{\text {excess }}=$ $\left(\bar{\Pi}-\bar{\Pi}_{0}\right) /\left(\phi g_{0}(2 ; \phi)\right)$, shown in Figure 6.7. This scaling collapses the data for all volume fractions to a large extent but there is still some $\phi$ dependence in the data.

The dependence of the stress on volume fraction can be seen more clearly in the bulk viscosity $\overline{\kappa^{P}}$ shown in Figure 6.8 and computed using (6.7), as the $P e$ dependence has been scaled out. The bulk viscosity plateaus to a constant limiting value in the limit of large $P e$ for all volume fractions. For smaller values of $P e$ there is a decrease in $\kappa^{P}$ for $\phi<0.2$ as $P e$ increases, indicating compression thinning of the bulk viscosity, but for higher $\phi$ the bulk viscosity is actually smaller at lower values of $P e$. The rate of change of volume fraction is exponentially faster at higher concentrations so there is progressively less time for the boundary layer to form as $\phi$ increases. There would also be some effect due to the finite cell size on which periodic boundary conditions are applied. Even though only the contact value of the pair distribution function $g(2 ; \phi)$ is needed to determine the pressure, the entire boundary layer must be set up correctly in order to get the correct value for $g(2 ; \phi)$. At high values of $P e$ the size of the boundary layer is significantly smaller than the cell length, so the periodic boundary conditions do not interfere with the formation of the boundary layer. The cell length is smaller for higher volume fractions, hence at intermediate $P e$ the boundary layer formation takes place correctly for small $\phi$ but not for larger values of $\phi$. For even smaller $P e$ the size of the boundary layer is too large for the simulation cell and the time required to reach steady state is also very long compared to the rate at which $\phi$ changes. Thus the expected compression thinning of the bulk viscosity at small $P e$ is not seen in our simulation results, except to some extent for $\phi<0.2$ and $1 \leq-P e \leq 10$.

The bulk viscosity normalized with $\phi^{2} g_{0}(2 ; \phi)$ to scale out the $\phi$ dependence is shown in Figure 6.9 along with the analytical curve from dilute theory. The data for $\phi=0.1$ is in excellent agreement with the dilute-theory prediction for $-P e \geq 10$. For smaller values of $P e$ it deviates from the dilute-theory curve as discussed above. We shall consider only the high $\mathrm{Pe}$ limit for scaling the bulk viscosity because it captures the correct physical behavior. For higher $\phi$ there is a mono- 
tonic increase with $\phi$ in the scaled bulk viscosity. The variations are small for $\phi \leq 0.3$ but increase rapidly for higher $\phi$. This behavior is reminiscent of the temporal scaling of the pressure autocorrelation function in equilibrium Brownian Dynamics simulations discussed in Chapter 5 §5.3.3. The increase in bulk viscosity can be attributed the hindering effect of surrounding particles at higher concentrations, which increases the diffusive time scale of the particles and the rate at which fluctuations in the stress are dissipated, thus increasing the bulk viscosity. Since the excess stress comes mostly from the boundary layer at contact, the entropic effect of surrounding particles outside the boundary layer does not decrease the diffusive time scale for small $\phi$ as it did in the equilibrium simulations. Accordingly, we define the diffusive time-scale

$$
\hat{D}_{N H}(\phi)=\left\{\begin{array}{ll}
1 & \text { if } \phi \leq 0.3 \\
D_{\infty}^{s}(\phi) / D_{\infty}^{s}(0.3) & \text { if } \phi>0.3
\end{array},\right.
$$

where we use the approximation $D_{\infty}^{s}(\phi)=[1+2 \phi g(2 ; \phi)]^{-1}$ for the nondimensional long-time selfdiffusivity proposed by Brady [1994] as it produced the best collapse of the simulation data. The scaled bulk viscosity defined as

$$
\hat{\kappa}^{P}=\frac{\kappa^{P}}{\eta} \frac{\hat{D}_{N H}(\phi)}{\phi^{2} g_{0}(2 ; \phi)}
$$

plotted as a function of the scaled Péclet number $\hat{P e}=P e / \hat{D}_{N H}(\phi)$ is shown in Figure 6.10. The data for all volume fractions collapses with this scaling in the high $\mathrm{Pe}$ region. For smaller values of $\mathrm{Pe}$ the scaled bulk viscosity data decreases with increasing $\phi$ because of the decreasing simulation cell size and the longer times needed to reach steady state. The above results are all from simulations with $N=1000$ particles. Simulations were also performed with 100 particles to serve as a check because the cell size would be smaller with fewer particles, and the scaled results are shown in Figure 6.11. Note that the data for $\phi \leq 0.2$ deviates from the dilute-theory curve at larger $P e$ with 100 particles than with 1000 particles, thus confirming that the cell size does influence the microstructure. For an infinite system at steady state we expect $\hat{\kappa}^{P}$ to be closer to the dilute-theory curve for all values of $\phi$ and $P e$. Thus the hard sphere bulk viscosity for concentrated systems scales as the number of collisions taking place and the increase in the diffusive time-scale due to 'caging' effects at high concentrations. 


\subsection{Accelerated Stokesian Dynamics}

The influence of hydrodynamic interactions on the bulk viscosity in compression was studied using ASD simulations with 100 particles. The full far-field computation was performed for determining the particle velocities and stress due to the imposed compression flow, while the ASDB-nf approximation Banchio and Brady [2003] was employed for Brownian motion. The ASDB-nf method of is an $O(N)$ procedure where the Brownian forces arising from far-field interactions are approximated in a mean-field manner and only the near-field lubrication interactions are actually evaluated between particles. The far-field diffusivities used for the mean-field approximation are already corrected for an infinite system, hence the hydrodynamic interactions evaluated using this technique are independent of the number of particles in the simulation cell. Finite-size effects may appear only due to the periodic boundary conditions used for evaluating particle collisions. Conventional Stokesian Dynamics simulations with 27 particles were also performed to serve as a test for the simulation technique and to study the effect of having a smaller system size. The SD simulations explicitly account for all the far-field and near-field interactions.

The particle-phase stress $\left\langle\boldsymbol{\Sigma}_{p}\right\rangle$ can be written as

$$
\left\langle\boldsymbol{\Sigma}_{p}\right\rangle=-n k T \boldsymbol{I}+n\left[\left\langle\boldsymbol{S}^{E}\right\rangle+\left\langle\boldsymbol{S}^{B}\right\rangle+\left\langle\boldsymbol{S}^{P}\right\rangle\right]
$$

where $-n k T I$ is the osmotic pressure due to the thermal kinetic energy of the Brownian particles and $\left\langle\boldsymbol{S}^{E}\right\rangle,\left\langle\boldsymbol{S}^{B}\right\rangle$ and $\left\langle\boldsymbol{S}^{P}\right\rangle$ are the average hydrodynamic, Brownian and interparticle-force particle stresslets (symmetric first moment of the force distribution integrated over the particle surface). The direct hydrodynamic contribution to the stress due to the disturbance flows caused by the finite size of the particles in an imposed flow is given by $n\left\langle\boldsymbol{S}^{E}\right\rangle$, and it is directly proportional to the applied rate of deformation. The hydrodynamic contribution to the bulk viscosity obtained from the hydrodynamic stresslet is given by

$$
\kappa^{H}=\frac{n\left\langle S^{E}\right\rangle}{3\langle e\rangle},
$$

and in nondimensional form

$$
\overline{\kappa^{H}}=\frac{1}{4} \phi \frac{\left\langle\overline{S^{E}}\right\rangle}{P e},
$$


where $\overline{\kappa^{H}}=\kappa^{H} / \eta$ and $\left\langle\overline{S^{E}}\right\rangle=\left\langle S^{E}\right\rangle / 6 \pi \eta a^{3}$. In the presence of hydrodynamic interactions the particle surfaces never touch due to the strong lubrication forces near contact and accordingly $n\left\langle S^{P}\right\rangle=0$ while $n\left\langle S^{B}\right\rangle$ accounts for all of the Brownian stress. The hard sphere nature of the particles is preserved by the no-slip hydrodynamic boundary condition on the particle surface [Brady 1993a]. The Brownian contribution to the stress has a finite average value at equilibrium given by the equilibrium osmotic pressure due to the thermal motion of the particles. Deviations from the equilibrium value occur only when the microstructure is perturbed. The Brownian bulk viscosity contribution is given by

$$
\kappa^{B}=\frac{n\left\langle S^{B}\right\rangle-n\left\langle S^{B}\right\rangle^{e q}}{3\langle e\rangle},
$$

where the superscript $e q$ denotes the equilibrium value. In nondimensional form

$$
\overline{\kappa^{B}}=\frac{3}{4} \phi \frac{\left(\overline{\Pi^{B}}-\bar{\Pi}_{0}\right)}{P e},
$$

where $\overline{\kappa^{B}}=\kappa^{B} / \eta$ and $\overline{\Pi^{B}}=\left\langle S^{B}\right\rangle / 3 k T$ is the nondimensional Brownian contribution to the particlephase pressure.

Simulations were performed with $N=100$ particles for several values of $P e$ ranging from 0.1 to 1000, with 200 distinct runs for each value of $P e$. The simulations were performed with a time step of $\Delta t=10^{-3}$ for $P e \geq 1$ and $\Delta t=2 \times 10^{-3}$ for $P e<1$, starting with a volume fraction of $\phi=0.1$. The lubrication forces between particles near contact normally prevent particle overlaps, but any overlaps encountered due to the finite time-step used in simulation were resolved at each step. In case of a severe overlap the particles were moved back to their previous positions and the same step was attempted again but with a different set of Brownian forces. At high Pe the particles were prone to jamming beyond $\phi>0.45$. The iterative numerical inversion of the resistance matrix failed in such cases and the simulation run was stopped. The far-field contribution to the particle velocities changes negligibly for small changes in the particle positions. Hence the far-field computation was performed only at predetermined intervals, starting with an interval of 50 time steps in the beginning of the simulation and reducing the interval with increasing $\phi$ down to every 5 time steps for $\phi>0.45$. For conventional SD the compression simulations were able to go up to higher volume fractions $(\sim 0.6)$ because the matrix inversion is exact and the number of particles was small which made the 
matrices less stiff. Eventually the SD simulations would stop due to severe particle overlaps.

\subsubsection{Results and scaling}

The Brownian contribution to the particle-phase pressure due to interactions between the particles, given by $\overline{\Pi^{B}}-1$ is shown in Figure 6.12 as a function of the Péclet number for compression. The pressure asymptotes to its equilibrium value as $P e \rightarrow 0$ and in the $P e \rightarrow-\infty$ limit it asymptotes to a higher constant value. The Brownian stress comes from interactions between particles in the boundary layer near contact which is of size $O\left(|P e|^{-1}\right)$ while the accumulation near contact is $O(|P e|)$, hence the $P e$ scaling from the microstructural perturbation cancels out and the Brownian stress is therefore $O(1)$. The Brownian bulk viscosity $\overline{\kappa^{B}}$ (shown in Figure 6.13) is obtained as the excess pressure divided by the Péclet number, hence it decays as $|P e|^{-1}$ as $P e \rightarrow-\infty$. For small volume fractions $(\phi \leq 0.2)$ some compression thinning is observed at intermediate values of $1 \leq P e \leq 10$ as well as $\overline{\kappa^{B}}$ decreases with increasing $|P e|$, but for higher volume fractions the bulk viscosity is constant even at these intermediate values of $P e$. Variations with $\phi$ in the intermediate and small $P e$ regime are due to the smaller cell sizes and slower diffusive times for higher volume fractions as discussed in the previous section.

The bulk viscosity is expected to have primarily the same $\phi^{2} g_{0}(2 ; \phi)$ scaling as in BD simulations, except for an additional scaling with the short-time self-diffusivity $D_{0}^{s}(\phi)$ to account for the increasingly stronger hydrodynamic interactions with increasing $\phi$. Figure 6.14 shows the Brownian contribution to the bulk viscosity scaled with $\phi^{2} g_{0}(2 ; \phi) / D_{0}^{s}$ where $D_{0}^{s}$ is already nondimensionalized with the bare diffusivity $D$. There is excellent collapse of the data for $\phi \leq 0.35$ and for higher $\phi$ the bulk viscosity is also larger. Again, this indicates a slowing down of the diffusive time because of hindrance in the particle motion due to the 'caging' effect of surrounding particles at higher concentrations. Hence we define the scaled Brownian bulk viscosity as

$$
\hat{\kappa^{B}}=\frac{\kappa^{B}}{\eta} \frac{D_{0}^{s}(\phi) \hat{D}_{H}(\phi)}{\phi^{2} g_{0}(2 ; \phi)},
$$

where

$$
\hat{D}_{H}(\phi)= \begin{cases}1 & \text { if } \phi \leq 0.35 \\ D_{\infty}^{s}(\phi) / D_{\infty}^{s}(0.35) & \text { if } \phi>0.35\end{cases}
$$


and the scaled Péclet number is given by $\hat{P} e=P e / \hat{D}_{H}(\phi)$. A plot of $\hat{\kappa}^{B}$ vs $\hat{P} e$ is shown in Figure 6.15 and illustrates the much better collapse of the data with this scaling. The collapsed data is also very close to the analytical prediction from dilute theory and has the same scaling with $P e$.

At small $P e$ the agreement with the dilute-theory curve is worse because of finite cell size effects. For comparison, the scaled results from SD simulations with 27 particles are also shown in Figure 6.16. In SD simulations the full far-field interactions are computed for Brownian motion at each time-step, causing the far-field self-diffusivity to be hindered by the periodic nature of the interactions [Brady 1987; Banchio and Brady 2003]. Thus the diffusivity is artificially smaller in SD resulting in a higher bulk viscosity. The mean-field approximation for the far-field diffusivity in the ASDB-nf method eliminates the effect of periodicity on the self diffusivity.

The direct hydrodynamic contribution to the bulk viscosity is shown in Figure 6.17. Since the hydrodynamic stress is proportional to $P e, \kappa^{H}$ asymptotes to a constant in the high $P e$ limit. The simulation data for the hydrodynamic bulk viscosity can be collapsed for all $\phi$ by scaling the increase in $\kappa^{H}$ due to the compression flow $\left(\kappa^{H}-\kappa_{\infty}^{\prime}\right.$ ) with its value at equilibrium (given by $\kappa_{0}^{H}=\kappa_{\infty}^{\prime}-\frac{4}{3} \eta \phi$ ) times the equilibrium pair distribution function $g_{0}(2 ; \phi)$ as shown in Figure 6.18. The equilibrium value $\kappa_{0}^{H}$ is $O\left(\phi^{2}\right)$ and also accounts for hydrodynamic interactions, so this scaling is actually very similar to the scaling used for $\kappa^{B}$. The collapse for $\phi \leq 0.35$ is very good and in excellent agreement with the dilute theory in the high $P e$ limit, but for larger $\phi$ the scaled data is larger, indicating that the 'caging' effect at high concentrations influences $\kappa^{H}$ as well. Further scaling the data with $\hat{D}_{H}(\phi)$ from (6.17) collapses the data for all $\phi$ onto the dilute theory curve for large $P e$ as shown in Figure 6.19. For comparison, the scaled data from SD simulations (Figure 6.20) also collapses onto a single curve but is lower than the ASD data because of hindered hydrodynamic interactions between particles due to periodicity. As small $\mathrm{Pe}$ there is larger error in the data because of finite size effects and the inability of the microstructure to reach the infinite system steady state.

Finally, the total bulk viscosity from ASD simulations is shown in Figure 6.21. This is to be compared with Figure 6.8 from BD simulations. In both cases compression thinning is not observed at high volume fractions. The compression thinning in the ASD data for small $\phi$ is also very small because the Brownian contribution to the bulk viscosity $\kappa^{B}$ is small compared to the hard sphere bulk viscosity $\kappa^{P}$ to begin with. Hence shear thinning of $\kappa^{B}$ is less obvious in ASD simulations. Finally it is instructive to contrast the bulk viscosity at equilibrium with its value at very high rates 
of compression. Figure 6.22 shows the total bulk viscosity at equilibrium and at $P e=-1000$ as

a function of the volume fraction. Not only does the bulk viscosity have the same scaling with $\phi$ at both extremes of $P e$, the magnitude is also different only by a small factor for the entire range of $\phi$. Thus, although the bulk viscosity changes with the rate of compression, a reasonably good approximation can be had for all $P e$ from the equilibrium bulk viscosity.

\subsection{Effect of shear on the hydrodynamic bulk viscosity}

It has been shown through experiments and simulation that concentrated suspensions exhibit shear thickening at high rates of shearing [Gadala-Maria and Acrivos 1980; Brady and Morris 1997; Sierou and Brady 2002]. This phenomenon is attributed to the accumulation of particles along the compression axis caused by the strong shearing motion and depletion along the expansion axis. At a large Péclet number of shearing (which gives the relative strength of shearing and diffusion, $P e_{s h}=\dot{\gamma} a^{2} / D$ ) the particles are unable to diffuse back to the equilibrium configuration and so the microstructure is far from equilibrium. The strong lubrication forces in regions of high particle concentration prevent the particle clusters from breaking up. The clustering of particles increases the hydrodynamic stress in the system, thereby increasing the macroscopic dynamic viscosity of the suspension. If we consider the sudden expansion of a suspension with such a microstructure, the bulk viscosity would also be higher because the lubrication forces in the particle clusters would also increase the trace of the hydrodynamic stress resulting from the expansion flow. Therefore we expect the effect of strong shearing on the hydrodynamic bulk viscosity to be similar to the effect of shearing on the dynamic viscosity of the suspension.

Stokesian Dynamics simulations were performed with particle configurations at various volumefractions subjected to different rates of shearing. The hydrodynamic bulk viscosity was evaluated and averaged over all the configurations realized during the steady shear flow to obtain the macroscopic bulk viscosity for a strongly sheared suspension. The results for various volume-fractions and Péclet numbers of shearing are shown in Figure 6.23. It is evident that there is an increase in the hydrodynamic bulk viscosity at higher volume fractions and high $P e_{s h}$, as expected. In order to isolate the effect of shearing on the bulk viscosity we subtract the equilibrium hydrodynamic bulk viscosity and also scale it with the same. The effect of shearing on the hydrodynamic suspension 
shear viscosity is isolated in the same way. Thus, the normalized effect of shearing on the bulk viscosity (shown in Figure 6.24) is given by

$$
\overline{\kappa^{H}}=\frac{\kappa^{H}\left(P e_{s h}\right) / \kappa^{H}(0)-1}{\eta^{H}\left(P e_{s h}\right) / \eta^{H}(0)-1} .
$$

The simulation results for all $P e_{s h}$ collapse onto a single curve with this normalization, which goes to show that the effect of shearing on the bulk viscosity is the same as the effect of shearing on the dynamic viscosity. The change in the microstructure is identical for both quantities so the rate at which they increase is also the same. The collapse for $P e_{s h}<10$ is not good because of the smaller increase in $\kappa^{H}$ and therefore higher error in $\overline{\kappa^{H}}$. At smaller concentrations too there is more spread in the data because of smaller variations in $\kappa^{H}$ and $\eta^{H}$. Specifically, the relative increase in the hydrodynamic bulk viscosity is approximately four times the relative increase in the hydrodynamic shear viscosity for all values of $P e_{s h}$ at all concentrations.

Such a correlation can be used to estimate the bulk viscosity of a sheared suspension from the known dynamic viscosity of the suspension. It must be possible to obtain a similar correlation for expansion of only the particle phase as well. In most practical situations a suspension would undergo shearing in addition to expansion, so it is important to consider the effect of shearing on the effective bulk viscosity. At high shear rates the Brownian motion of the particles is overwhelmed by the imposed shear flow, so the Brownian contribution to the bulk viscosity is also very small. Therefore the hydrodynamic contribution to the bulk viscosity is most relevant in this case.

\subsection{Conclusions}

The work presented in this chapter is the first ever application of the Stokesian Dynamics paradigm to compression of a suspension. The simulation results were verified by using two different techniques, namely Stokesian Dynamics and Accelerated Stokesian Dynamics, and the results from both are in good agreement. We were also able to successfully determine the scalings that can be used to predict the bulk viscosity at high concentrations from the dilute analysis with reasonable accuracy. The scaling gives valuable insight into the interplay of physical processes that determine the microstructure and stress in compression. The size of boundary layer, and therefore the rate of 
compression, determines how long the system would take to reach a steady state and thus affects the magnitude of the bulk viscosity. The long-time self-diffusivity sets the time scale of relaxation at high volume fractions and the dissipation of stress outside the boundary layer is not important.

In most practical situations the compression flow may not last long enough to achieve steady state. The results obtained here give the upper bound for the bulk viscosity in that case. The actual bulk viscosity would be somewhere between the equilibrium value determined in the previous chapter and the high $P e$ limit. In most practical applications, the suspension would also undergo shear in addition to expansion. The bulk viscosity for a small rate of expansion in a steadily sheared suspension is relevant in such flow conditions. If the compression flow lasts long enough to distort the steady sheared microstructure there would be an additional Brownian contribution to the bulk viscosity not determined here. At small rates of shearing and compression the two flows can be superimposed but such a superposition would not be correct at high rates of shearing and compression. 


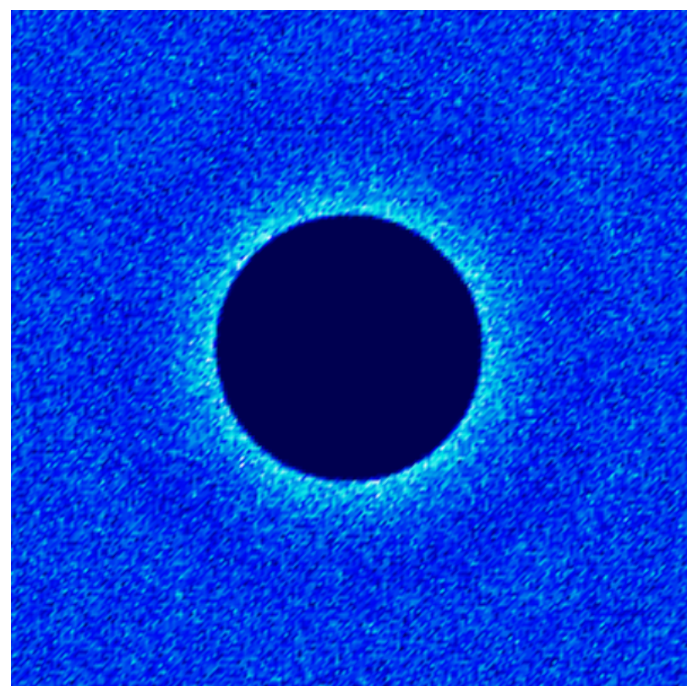

(a) $P e=-1, \phi=0.2$

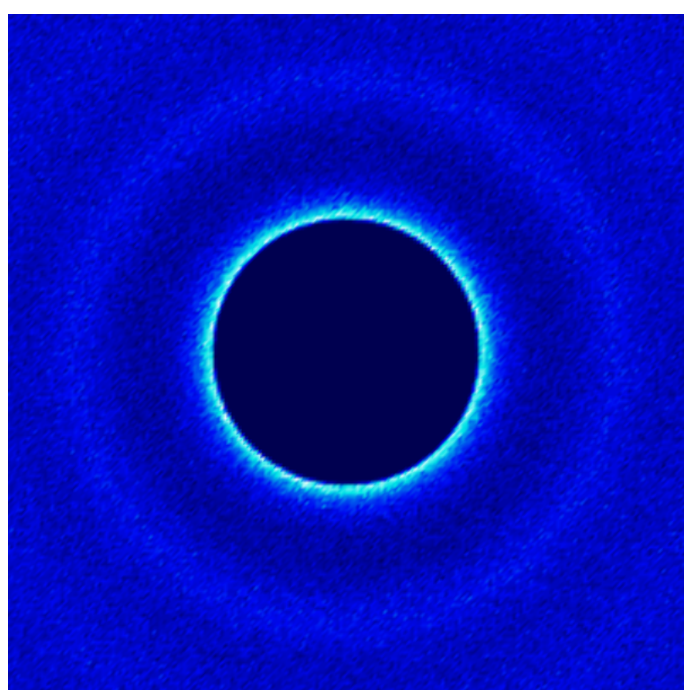

(c) $P e=-1, \phi=0.45$

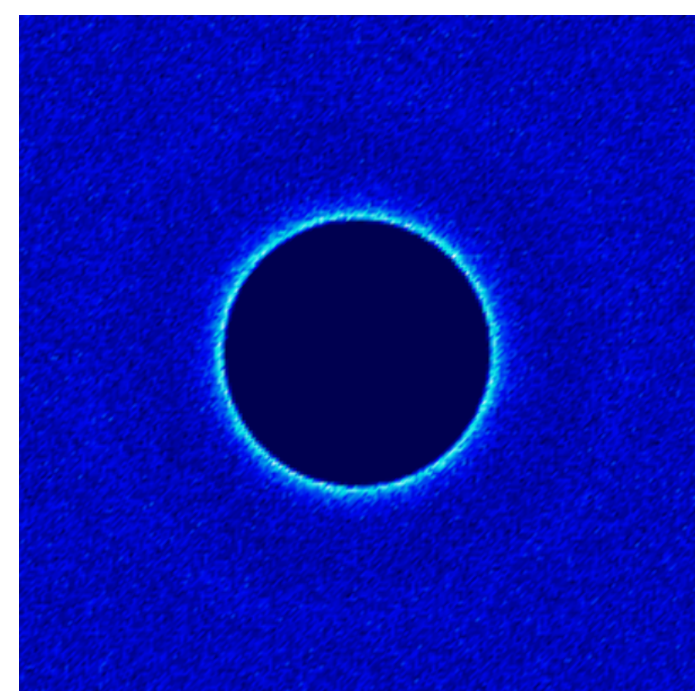

(b) $P e=-10, \phi=0.2$

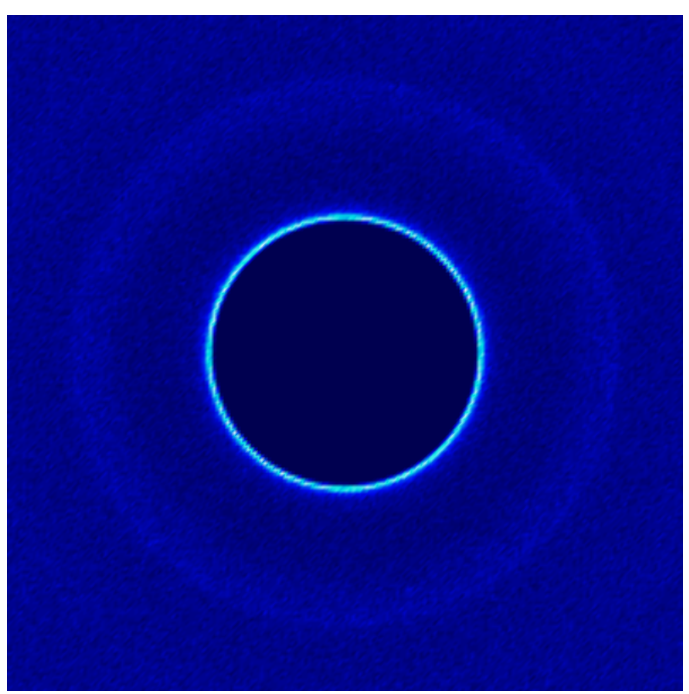

(d) $P e=-10, \phi=0.45$

Figure 6.1: Planar plots of the pair distribution function $g(r)$ obtained from Brownian Dynamics simulations for $\phi=0.2$ and $\phi=0.45$ at compression rates of $P e=-1$ and $P e=-10$. Lighter regions indicate accumulation of particles. 


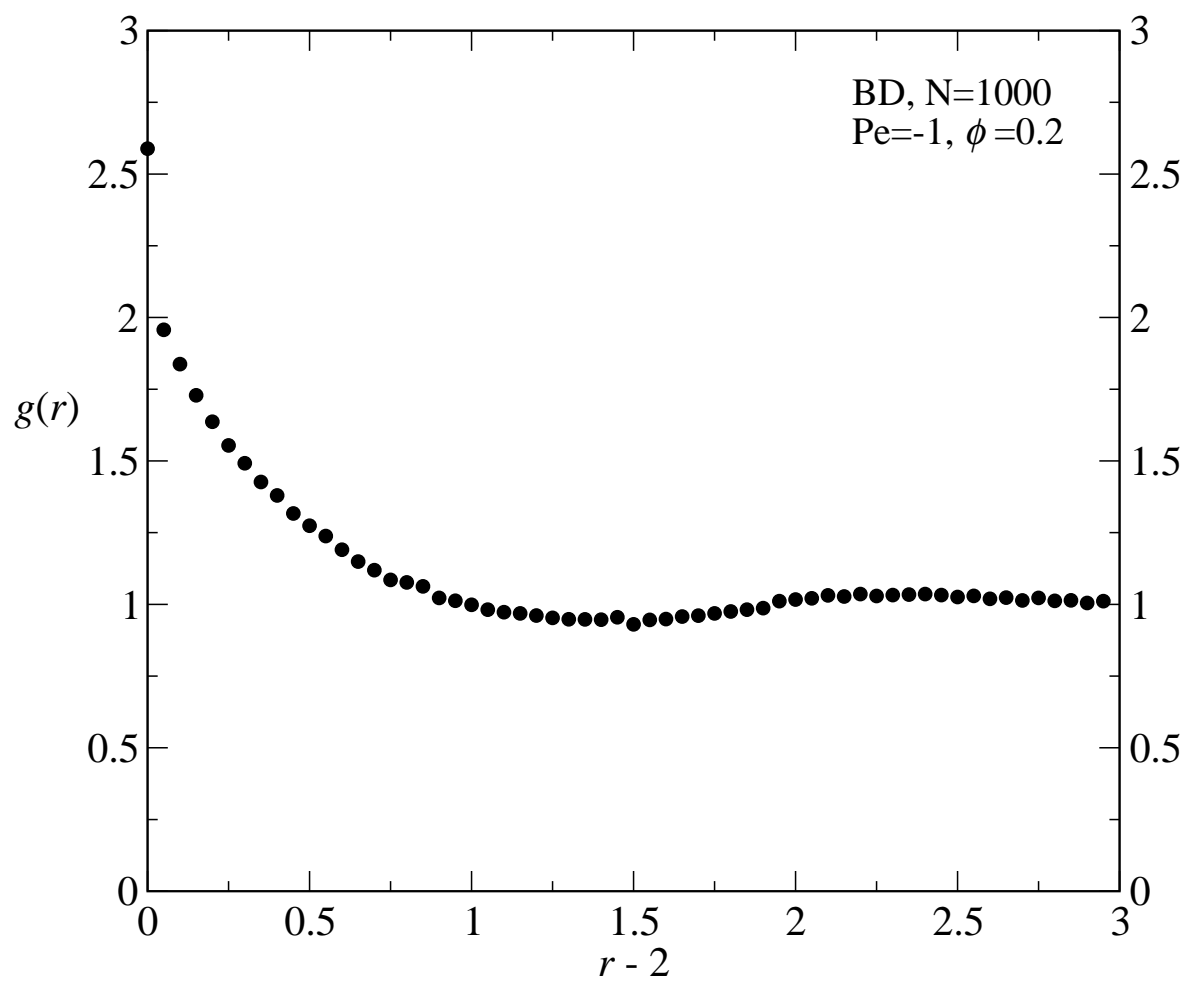

(a) $\mathrm{Pe}=-1$

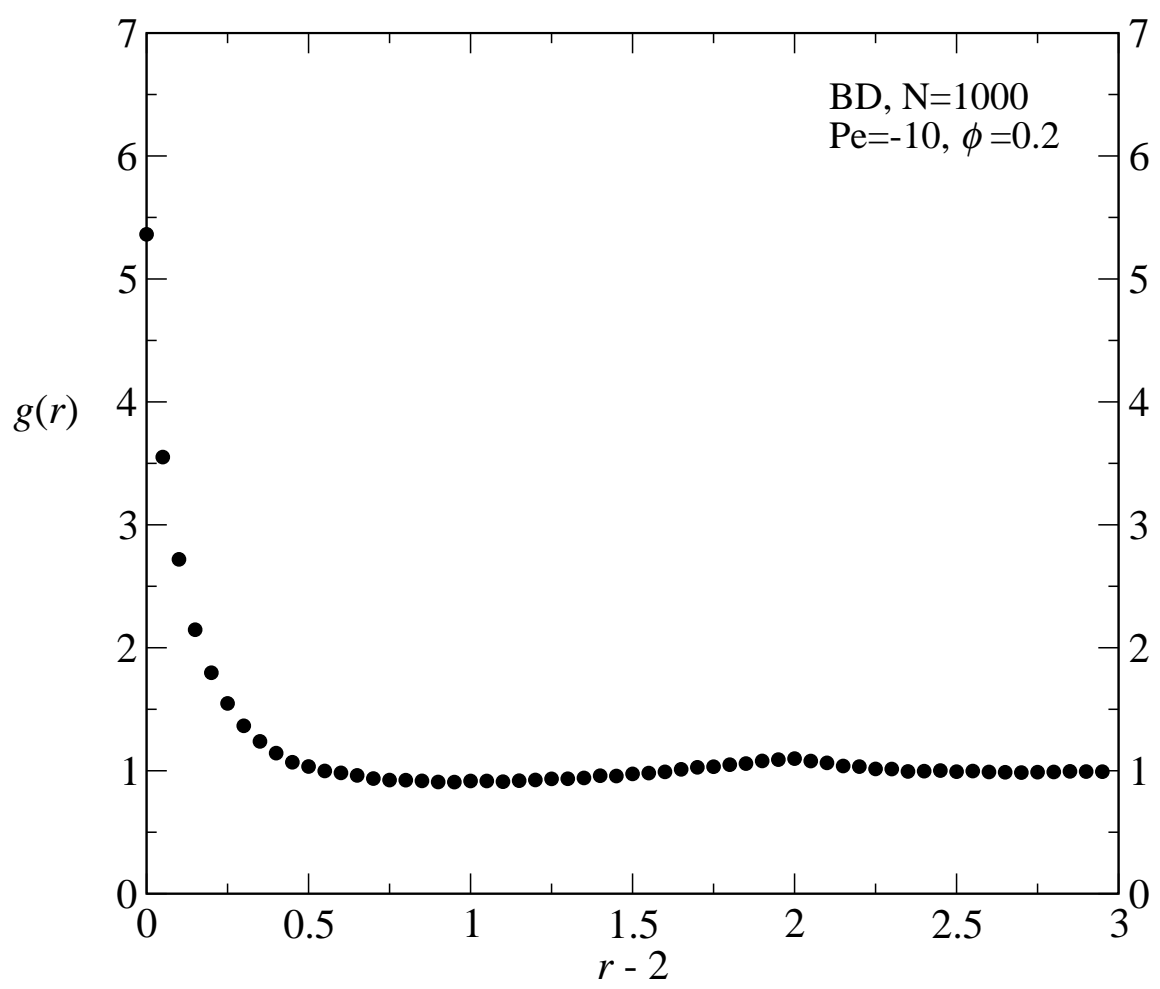

(b) $P e=-10$

Figure 6.2: Radial pair distribution in compression for $\phi=0.2$ obtained from Brownian Dynamics with $N=1000$, for rates $P e=-1$ and -10 . 


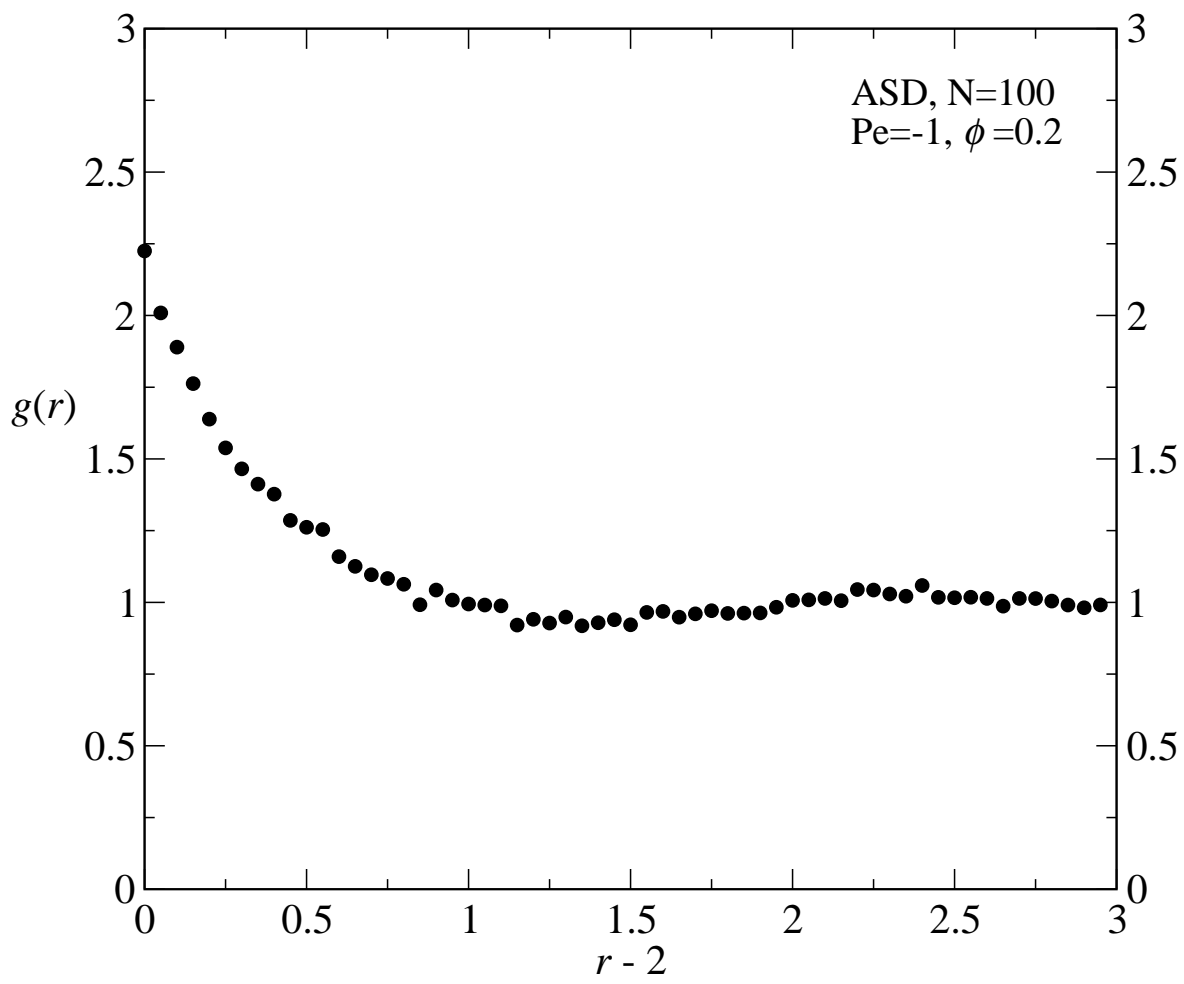

(a) $\mathrm{Pe}=-1$

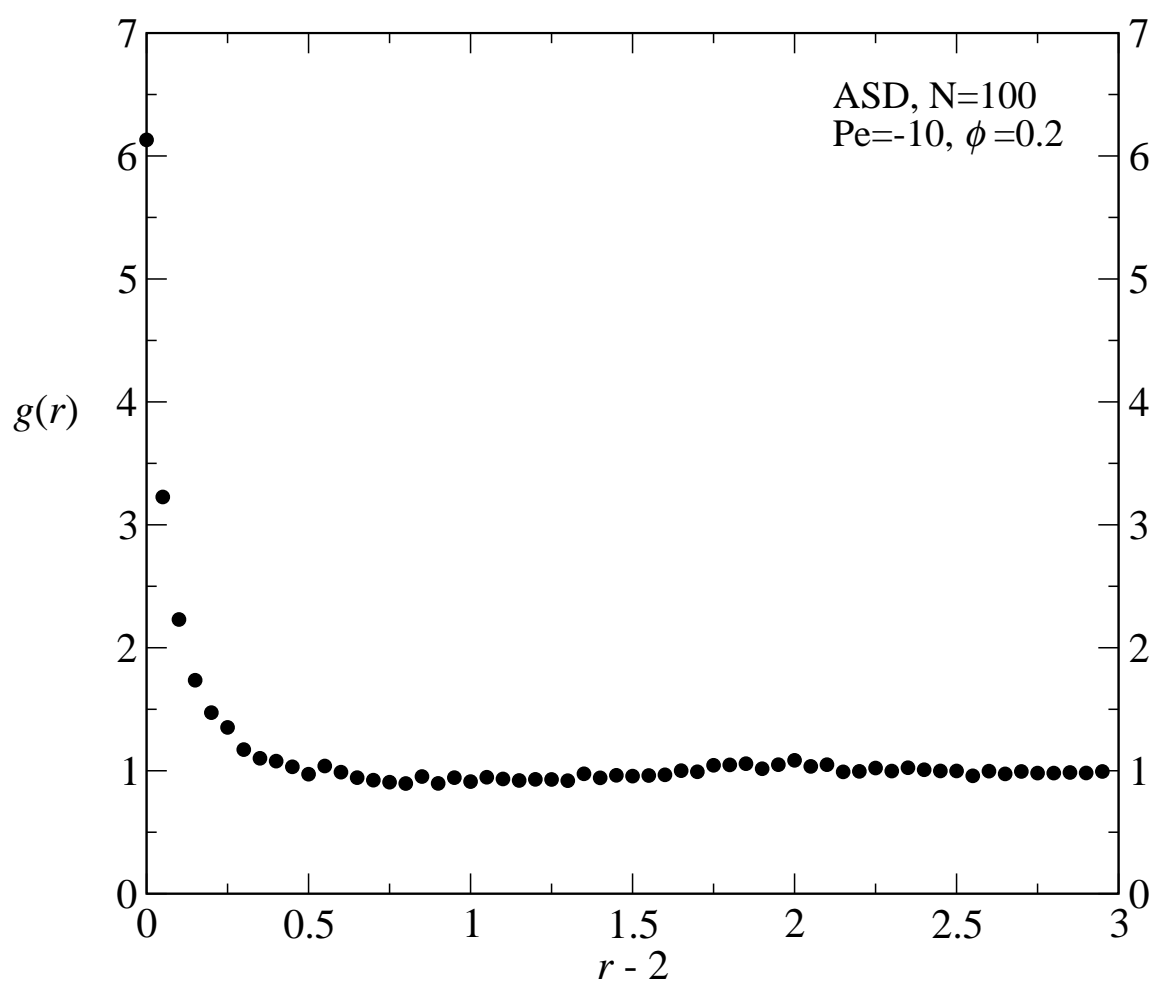

(b) $P e=-10$

Figure 6.3: Radial pair distribution in compression for $\phi=0.2$ obtained from Accelerated Stokesian Dynamics with $N=100$, for rates $P e=-1$ and -10 . 


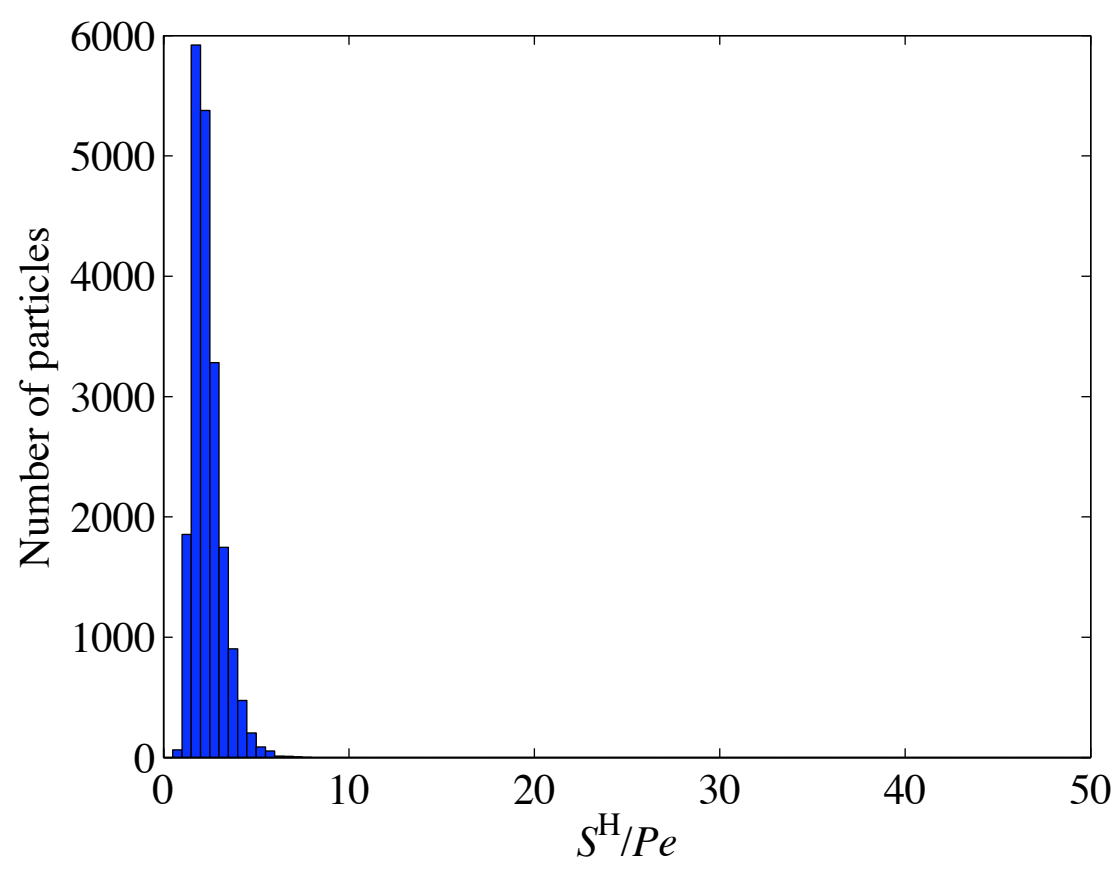

(a) $P e=-1$

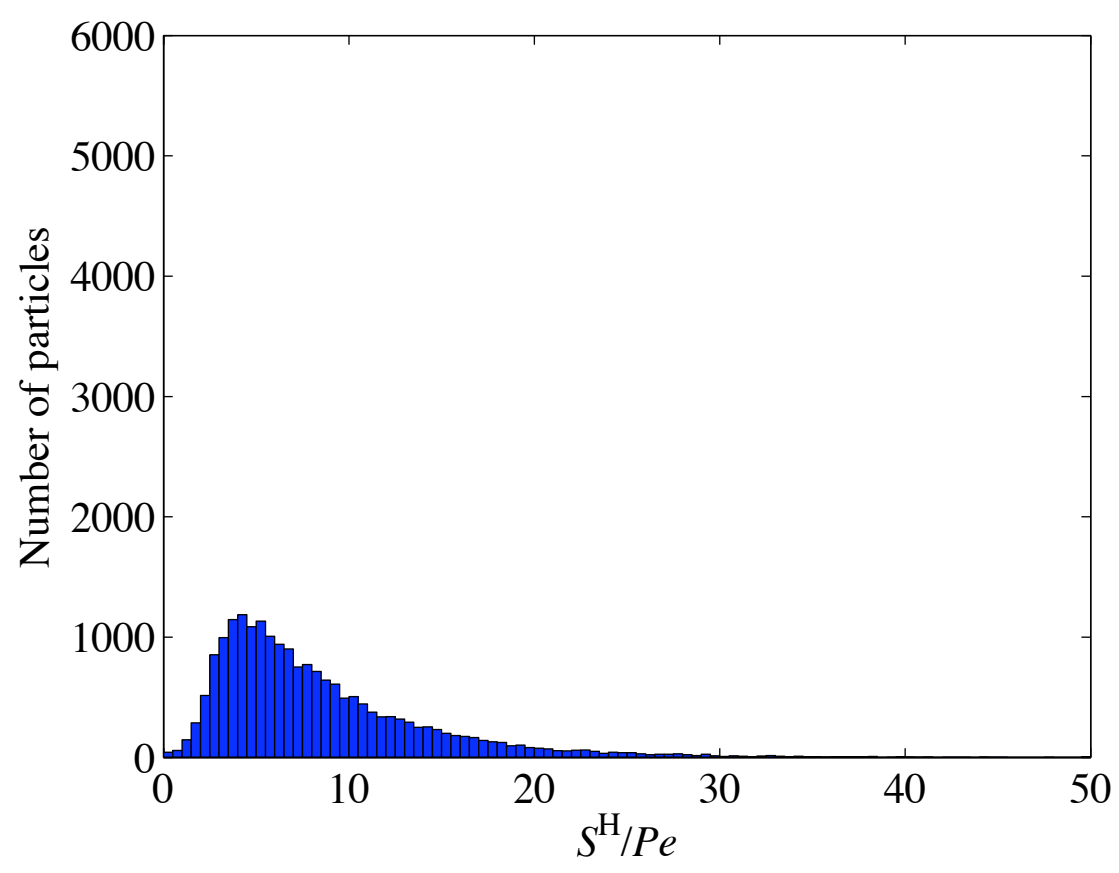

(b) $P e=-1000$

Figure 6.4: Histogram of scaled particle hydrodynamic pressure moments $\left\langle S^{H}\right\rangle / P e$ obtained from Accelerated Stokesian Dynamics with $N=100,200$ distinct runs, for rates $P e=-1$ (a) and -1000 (b). 

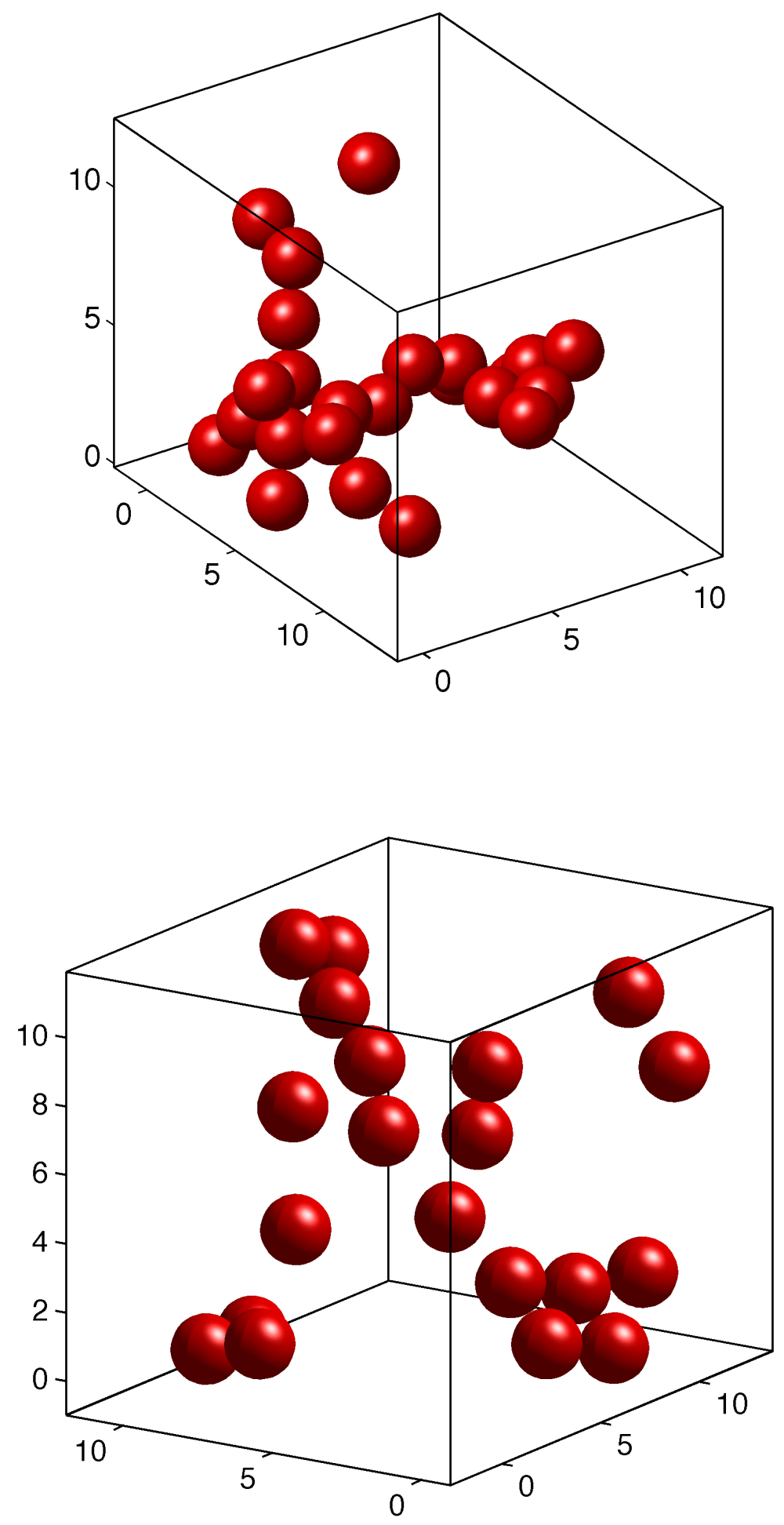

Figure 6.5: Two examples of particles with high hydrodynamic pressure moment $\left(\left\langle S^{H}\right\rangle / P e>10\right)$ forming clusters distributed randomly in space at high rate $(P e=-1000)$, obtained from Accelerated Stokesian Dynamics with $N=100$. 


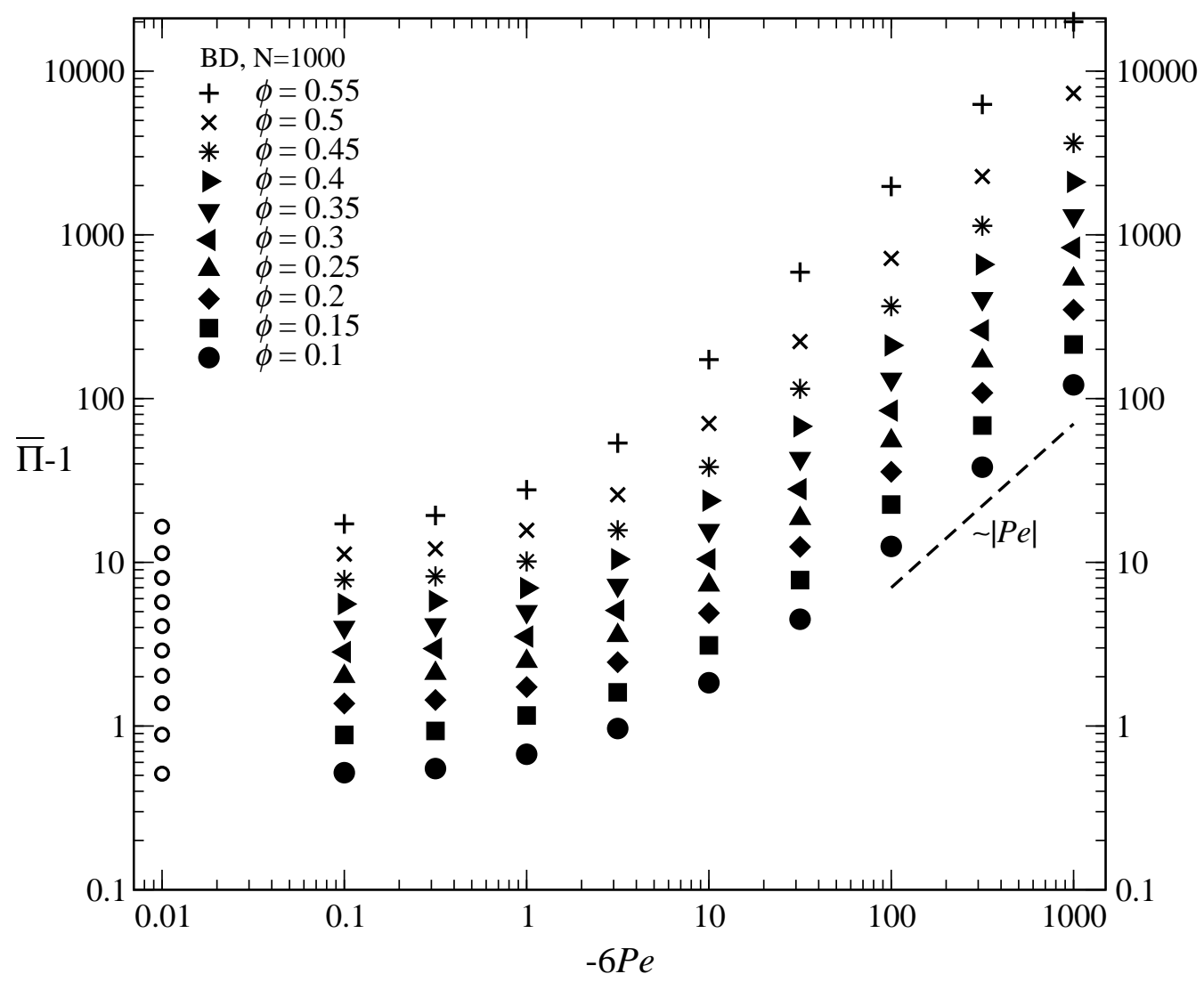

Figure 6.6: Nondimensional interparticle-force contribution to the pressure in compression vs Péclet number, from Brownian Dynamics with $N=1000$ for $\phi=0.1$ to $\phi=0.55$. The open circles denote the equilibrium particle contribution to the osmotic pressure. 


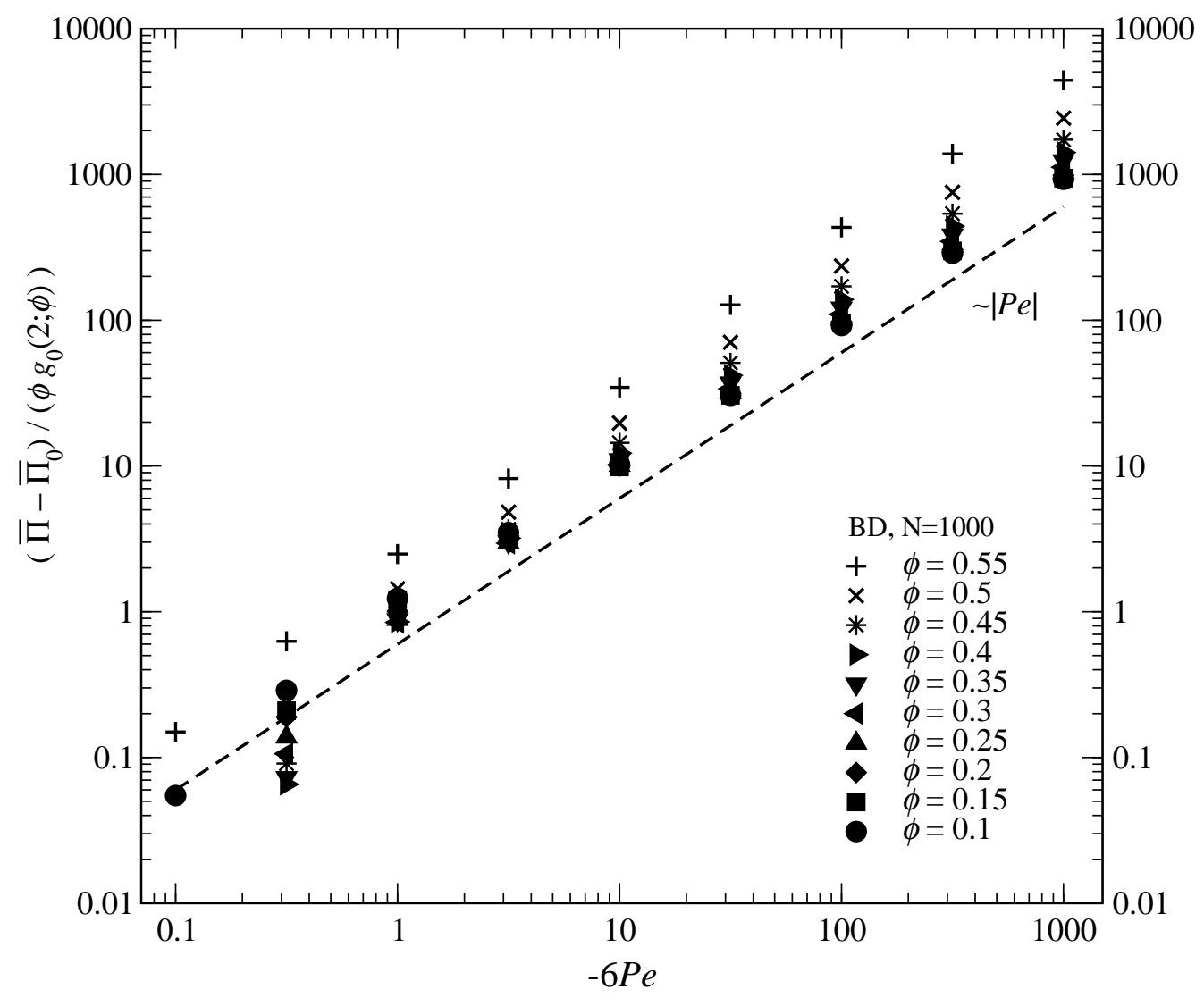

Figure 6.7: The excess interparticle-force contribution to the pressure in compression normalized with $\phi g_{0}(2 ; \phi)$ vs Péclet number, from Brownian Dynamics with $N=1000$ for $\phi=0.1$ to $\phi=0.55$. 


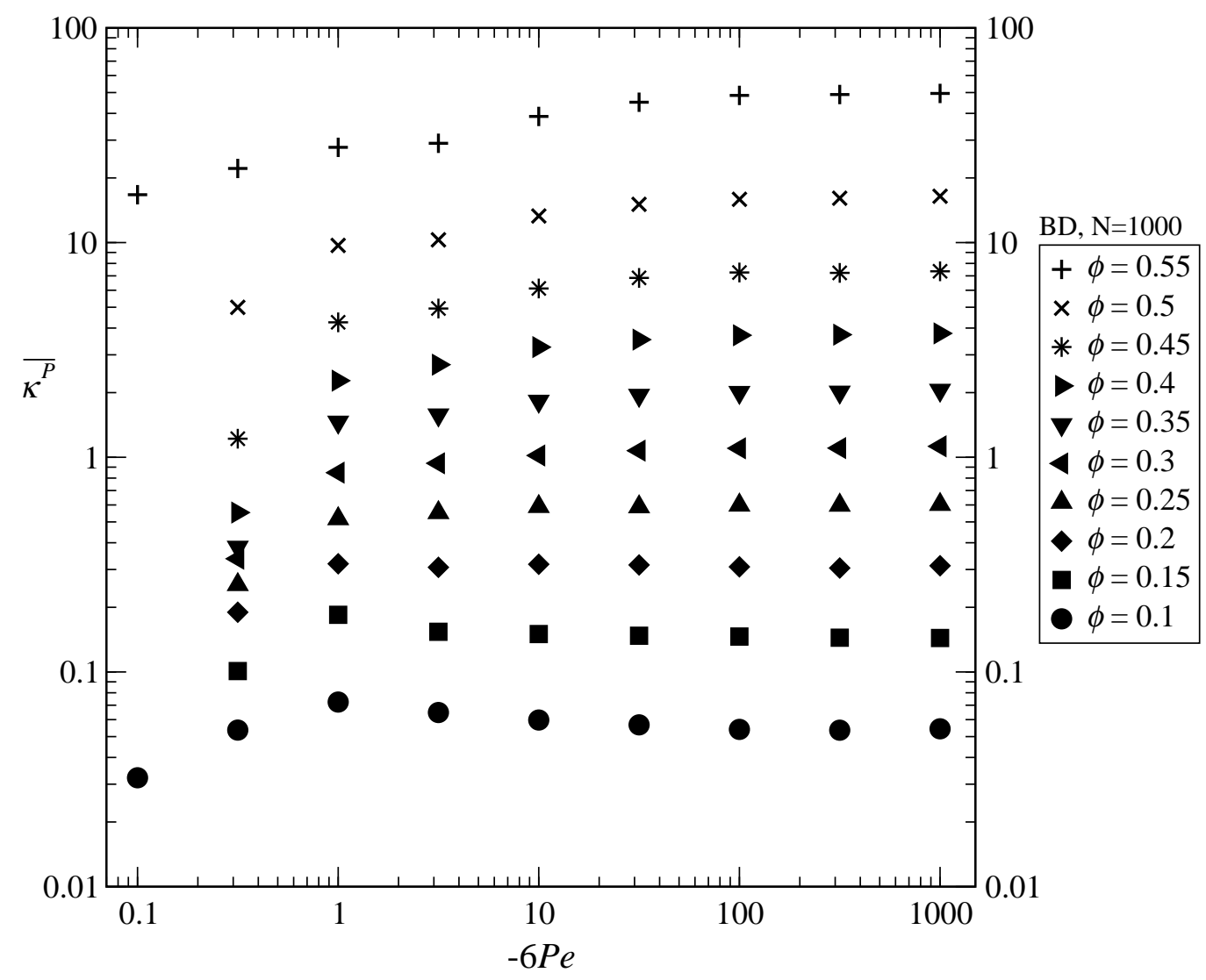

Figure 6.8: The nondimensional hard sphere bulk viscosity $\overline{\kappa^{P}}=\kappa^{P} / \eta$ vs Péclet number, from Brownian Dynamics with $N=1000$ for $\phi=0.1$ to $\phi=0.55$. 


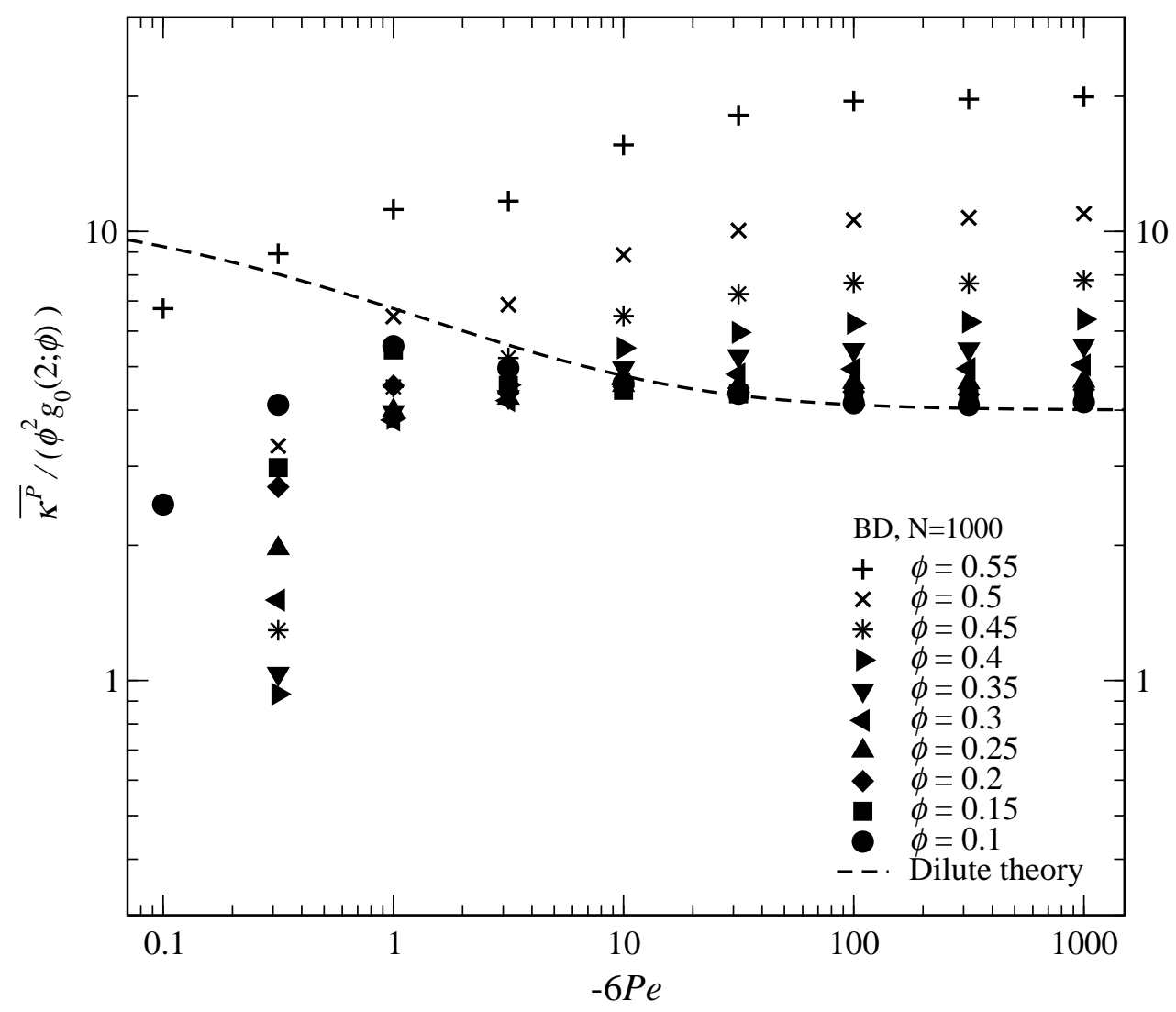

Figure 6.9: The nondimensional hard sphere bulk viscosity $\overline{\kappa^{P}}=\kappa^{P} / \eta$ normalized with $\phi^{2} g_{0}(2 ; \phi)$ vs Péclet number, from Brownian Dynamics with $N=1000$ for $\phi=0.1$ to $\phi=0.55$. 


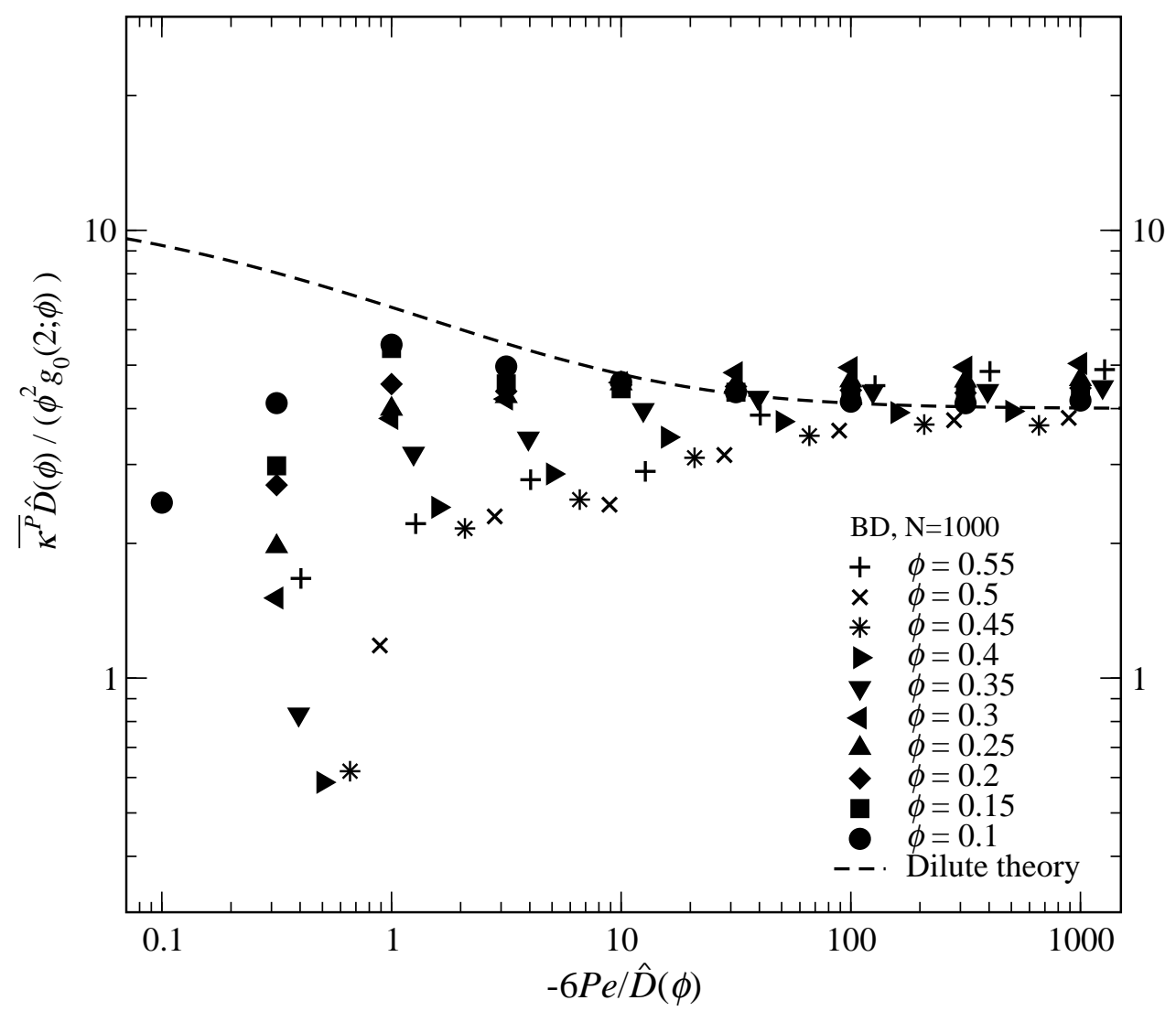

Figure 6.10: The nondimensional hard sphere bulk viscosity $\overline{\kappa^{P}}=\kappa^{P} / \eta$ normalized with $\phi^{2} g_{0}(2 ; \phi)$ and scaled with the time-scale for stress relaxation given by $\hat{D}_{N H}(\phi)$ vs the scaled Péclet number, from Brownian Dynamics with $N=1000$ for $\phi=0.1$ to $\phi=0.55$. 


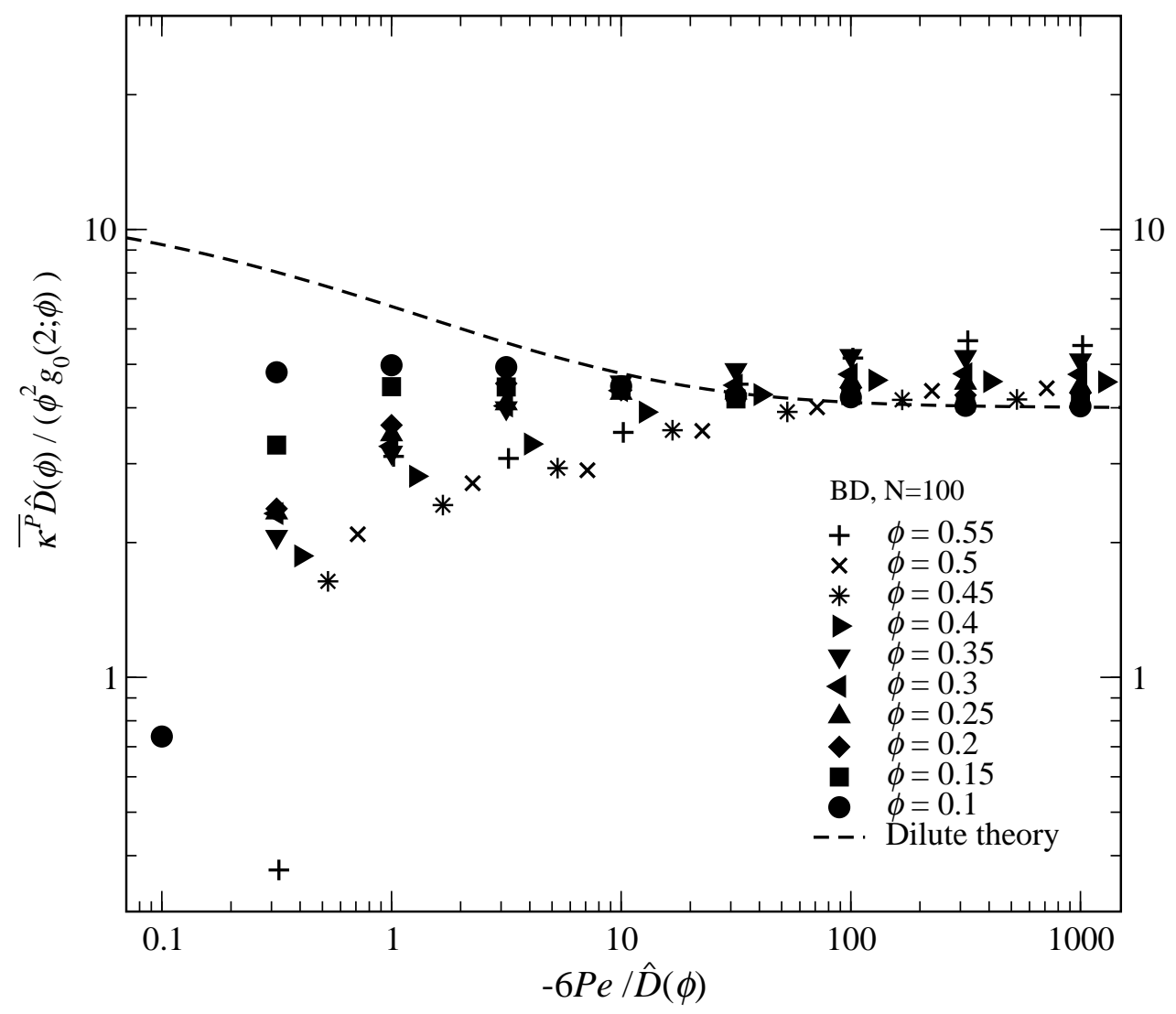

Figure 6.11: The nondimensional hard sphere bulk viscosity $\overline{\kappa^{P}}=\kappa^{P} / \eta$ normalized with $\phi^{2} g_{0}(2 ; \phi)$ and scaled with the time-scale for stress relaxation given by $\hat{D}_{N H}(\phi)$ vs the scaled Péclet number, from Brownian Dynamics with $N=100$ for $\phi=0.1$ to $\phi=0.55$. 


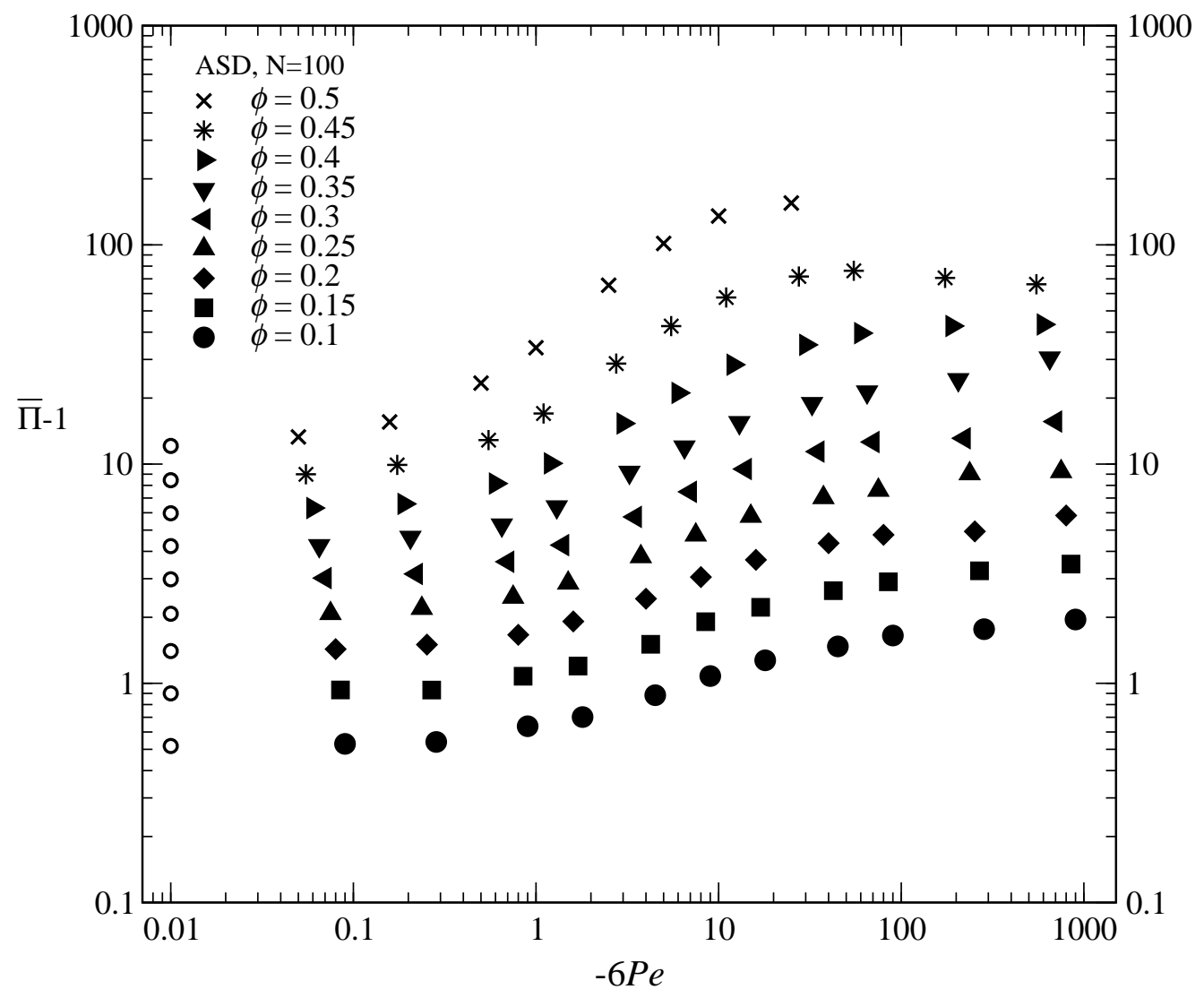

Figure 6.12: Nondimensional Brownian contribution to the pressure in compression vs Péclet number, from Accelerated Stokesian Dynamics - near field with $N=100$ for $\phi=0.1$ to $\phi=0.5$. The open circles denote the equilibrium particle contribution to the osmotic pressure. 


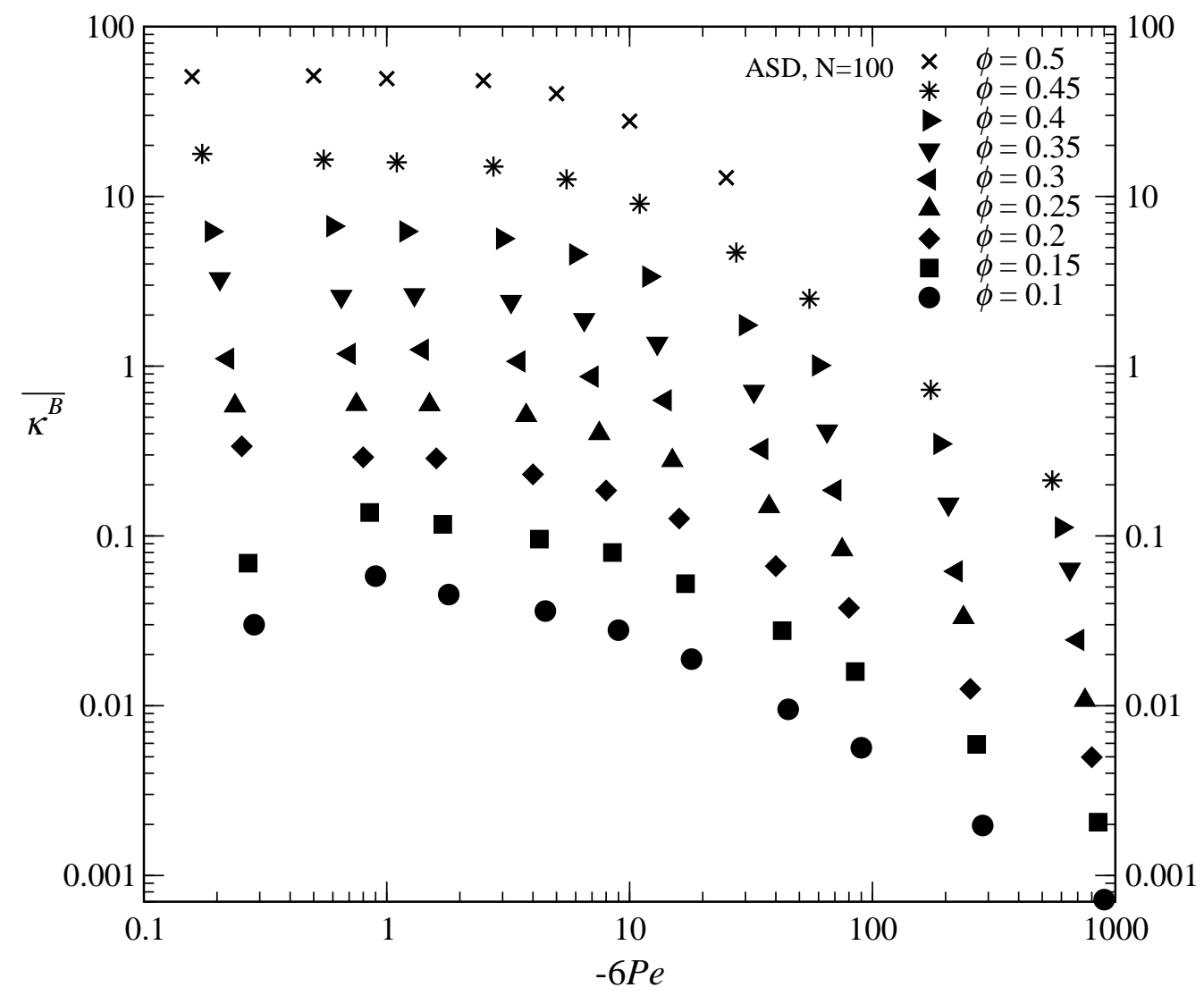

Figure 6.13: The nondimensional Brownian bulk viscosity contribution $\overline{\kappa^{B}}=\kappa^{B} / \eta$ vs Péclet number, from Accelerated Stokesian Dynamics — near field with $N=100$ for $\phi=0.1$ to $\phi=0.5$. 


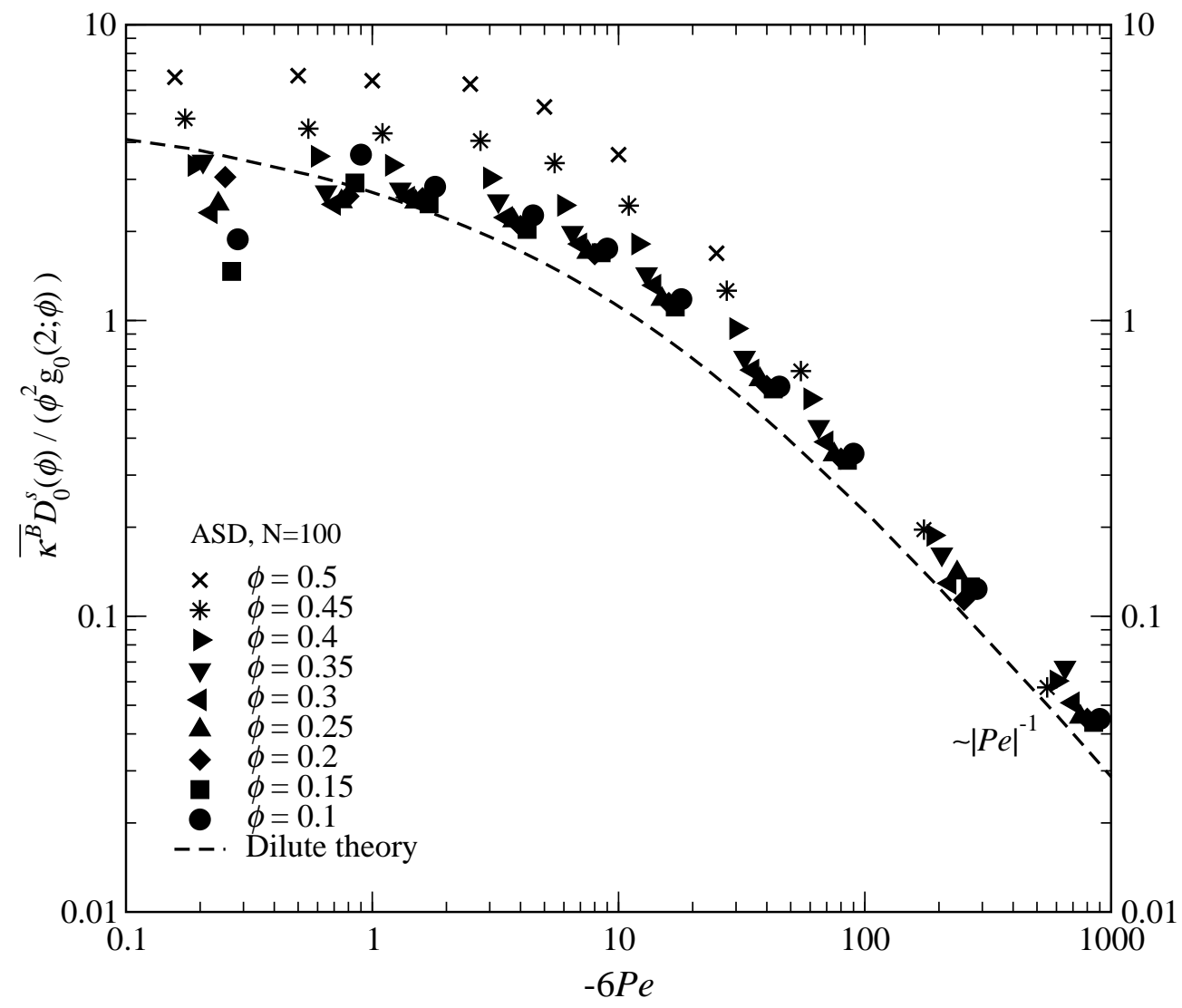

Figure 6.14: The nondimensional Brownian bulk viscosity contribution $\overline{\kappa^{B}}=\kappa^{B} / \eta$ normalized with $\phi^{2} g_{0}(2 ; \phi) / D_{0}^{s}(\phi)$ vs Péclet number, from Accelerated Stokesian Dynamics — near field with $N=$ 100 for $\phi=0.1$ to $\phi=0.5$. 


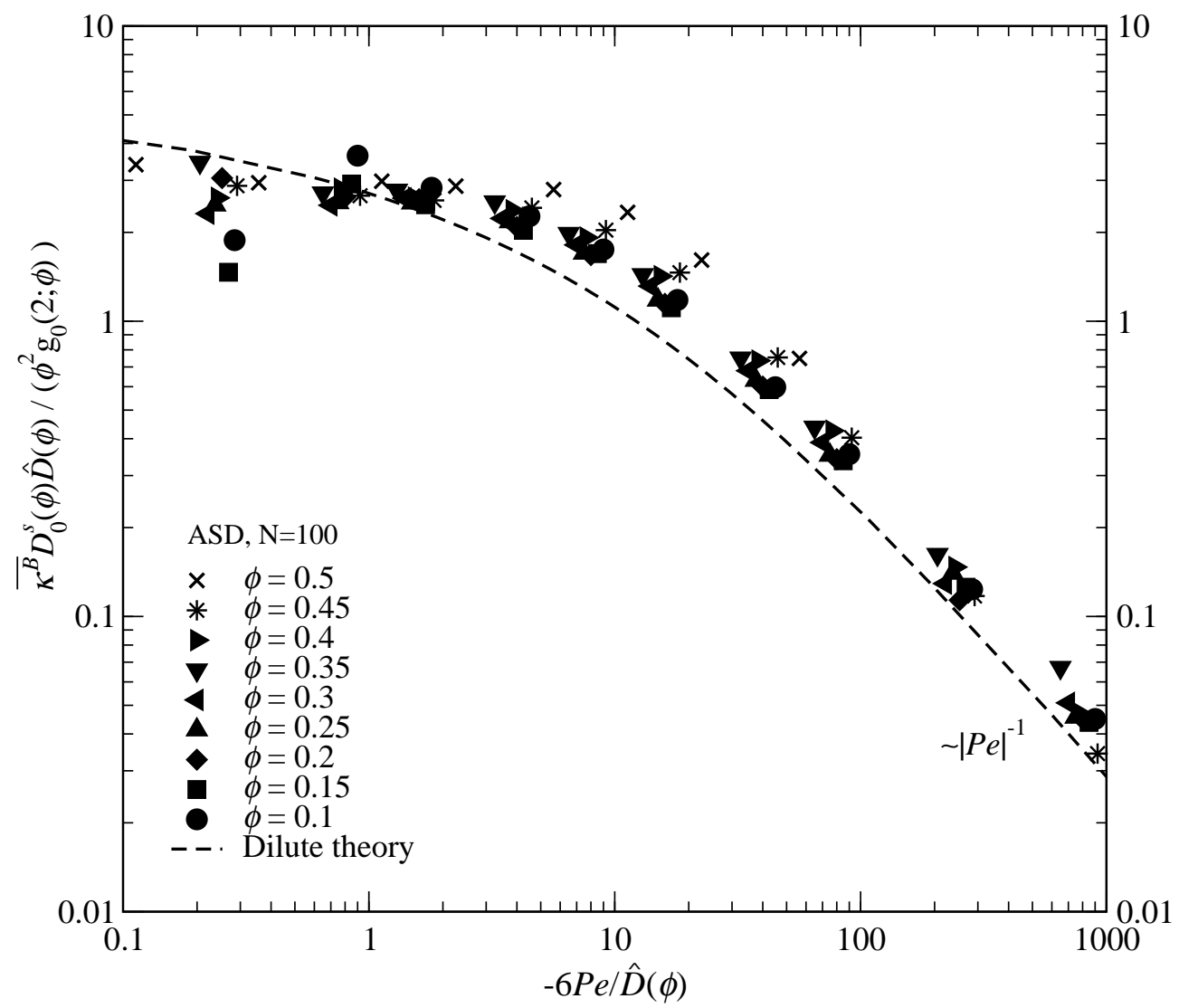

Figure 6.15: The nondimensional Brownian bulk viscosity contribution $\overline{\kappa^{B}}=\kappa^{B} / \eta$ normalized with $\phi^{2} g_{0}(2 ; \phi) / D_{0}^{s}(\phi)$ and scaled with the time-scale for stress relaxation given by $\hat{D}_{H}(\phi)$ vs the scaled Péclet number, from Accelerated Stokesian Dynamics - near field with $N=100$ for $\phi=0.1$ to $\phi=0.5$. 


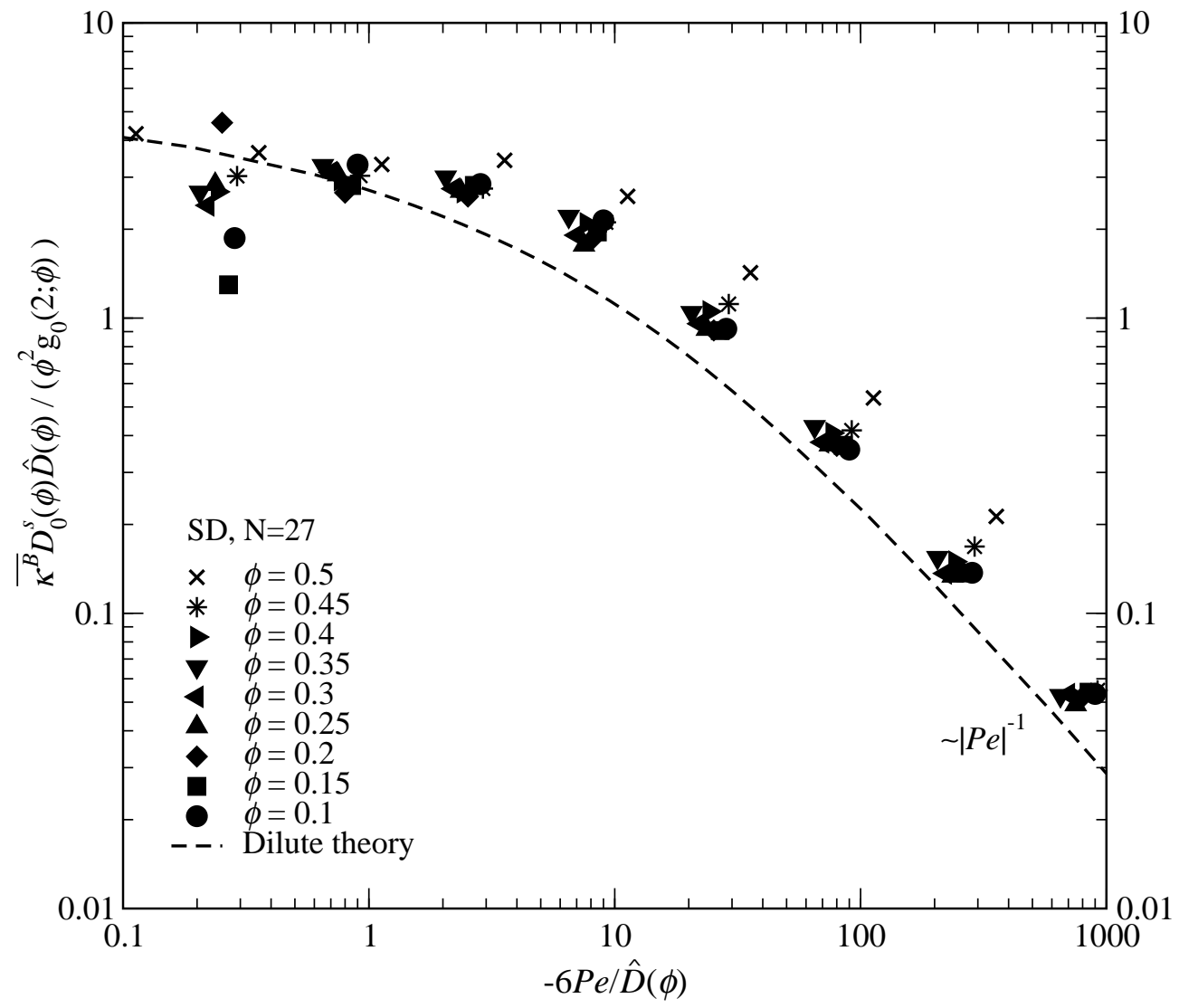

Figure 6.16: The nondimensional Brownian bulk viscosity contribution $\overline{\kappa^{B}}=\kappa^{B} / \eta$ normalized with $\phi^{2} g_{0}(2 ; \phi) / D_{0}^{s}(\phi)$ and scaled with the time-scale for stress relaxation given by $\hat{D}_{H}(\phi)$ vs the scaled Péclet number, from conventional Stokesian Dynamics with $N=27$ for $\phi=0.1$ to $\phi=0.5$. 


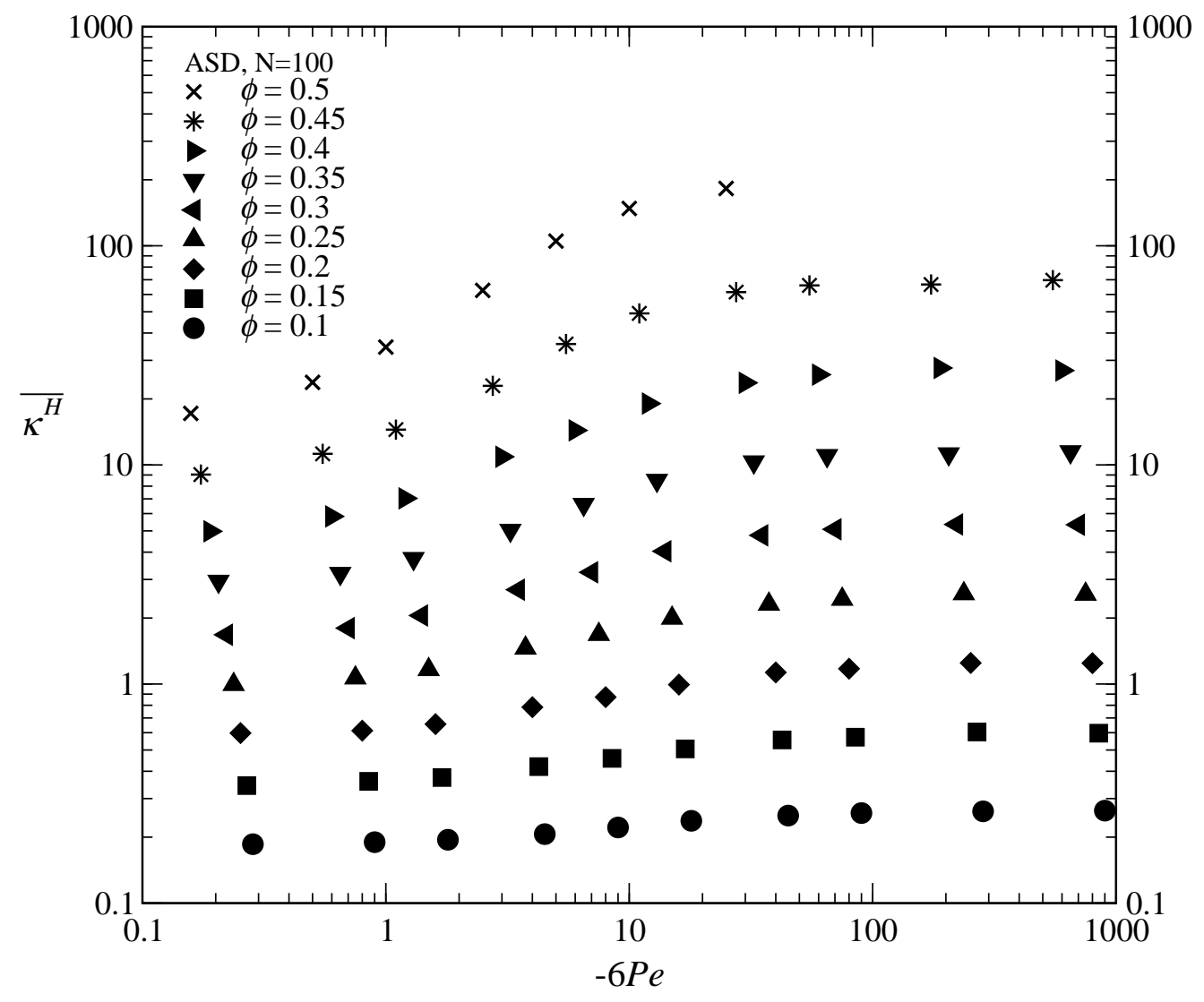

Figure 6.17: The nondimensional hydrodynamic bulk viscosity contribution $\overline{\kappa^{H}}=\kappa^{H} / \eta$ vs Péclet number, from Accelerated Stokesian Dynamics - near field with $N=100$ for $\phi=0.1$ to $\phi=0.5$. 


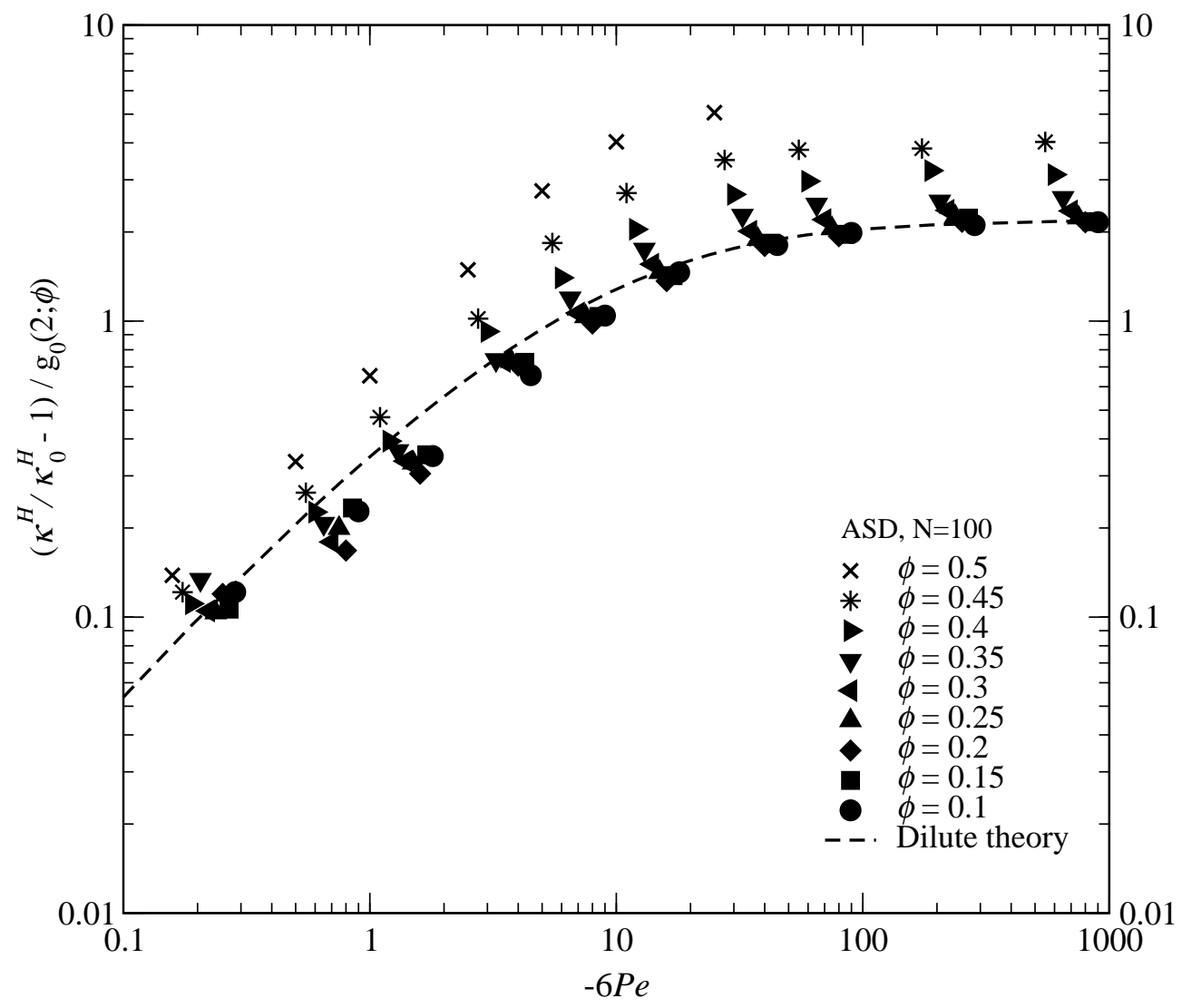

Figure 6.18: The normalized excess hydrodynamic bulk viscosity contribution $\left(\kappa^{H}(P e)-\kappa^{H}(0)\right) / \kappa^{H}(0)$ scaled with $g_{0}(2 ; \phi)$ vs Péclet number, from Accelerated Stokesian Dynamics — near field with $N=100$ for $\phi=0.1$ to $\phi=0.5$. 


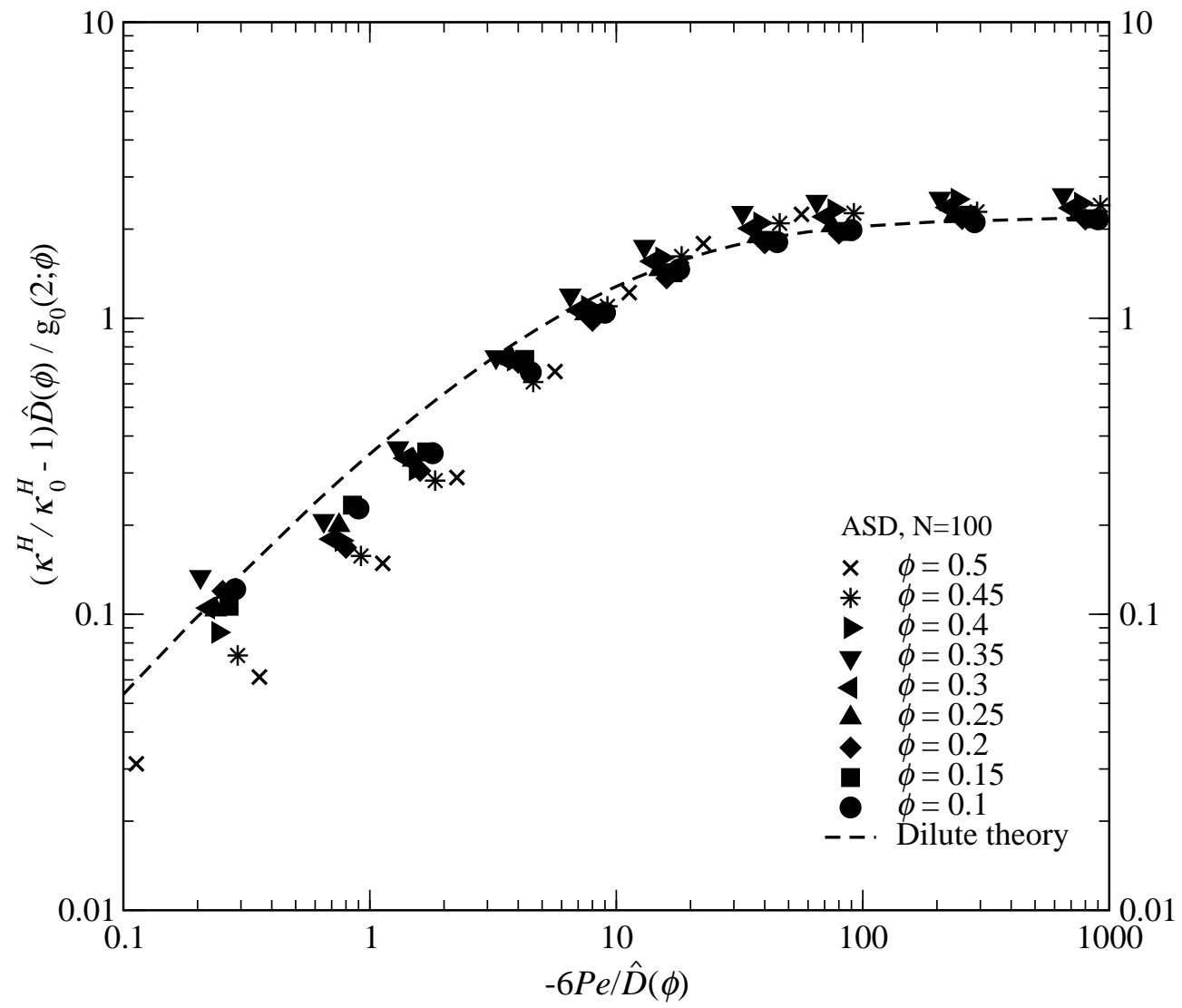

Figure 6.19: The normalized excess hydrodynamic bulk viscosity contribution $\left(\kappa^{H}(P e)-\kappa^{H}(0)\right) / \kappa^{H}(0)$ scaled with $g_{0}(2 ; \phi)$ and the time-scale for stress relaxation given by $\hat{D}_{H}(\phi)$ vs the scaled Péclet number, from Accelerated Stokesian Dynamics - near field with $N=100$ for $\phi=0.1$ to $\phi=0.5$. 


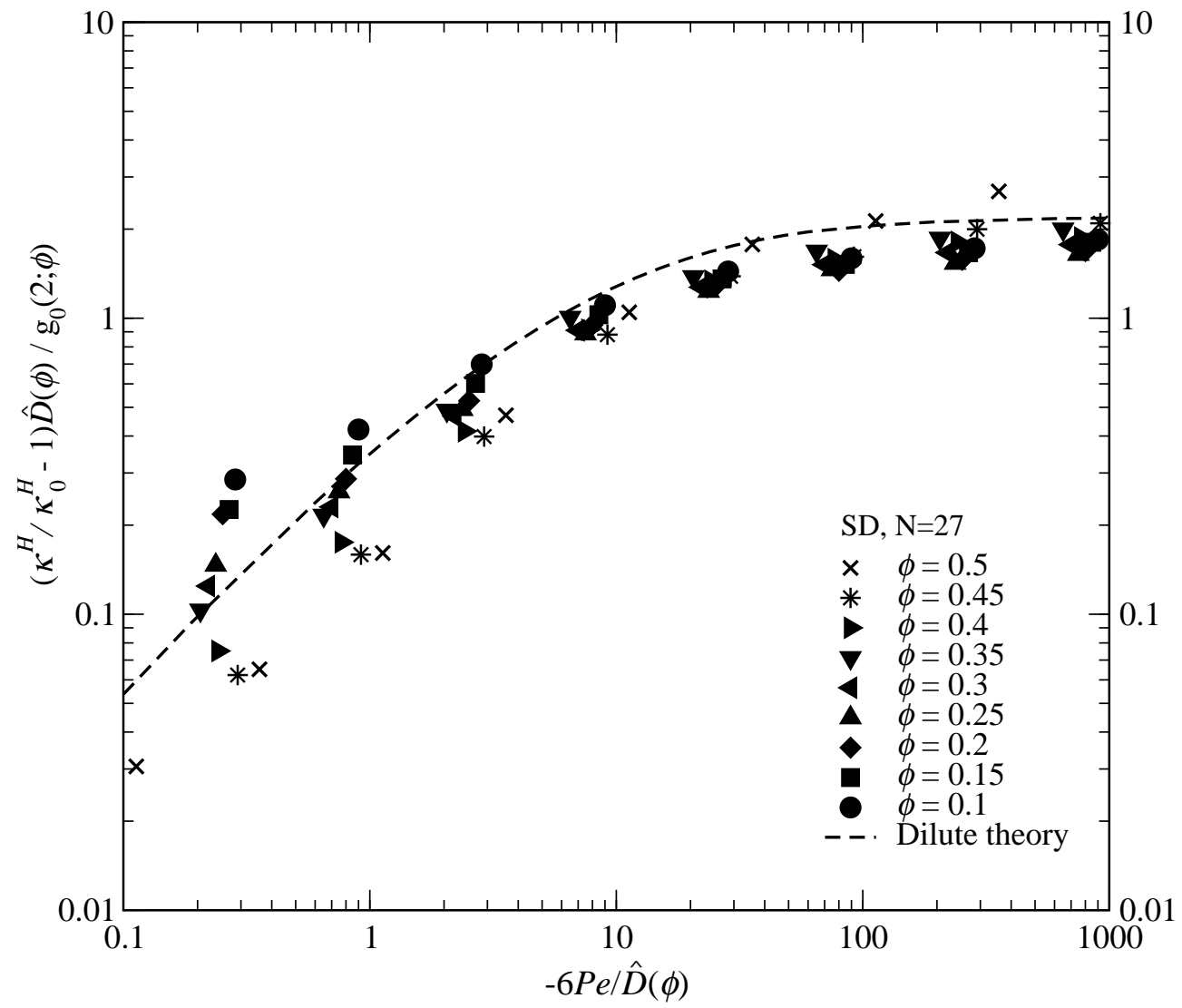

Figure 6.20: The normalized excess hydrodynamic bulk viscosity contribution $\left(\kappa^{H}(P e)-\kappa^{H}(0)\right) / \kappa^{H}(0)$ scaled with $g_{0}(2 ; \phi)$ and the time-scale for stress relaxation given by $\hat{D}_{H}(\phi)$ vs the scaled Péclet number, from conventional Stokesian Dynamics with $N=27$ for $\phi=0.1$ to $\phi=0.5$. 


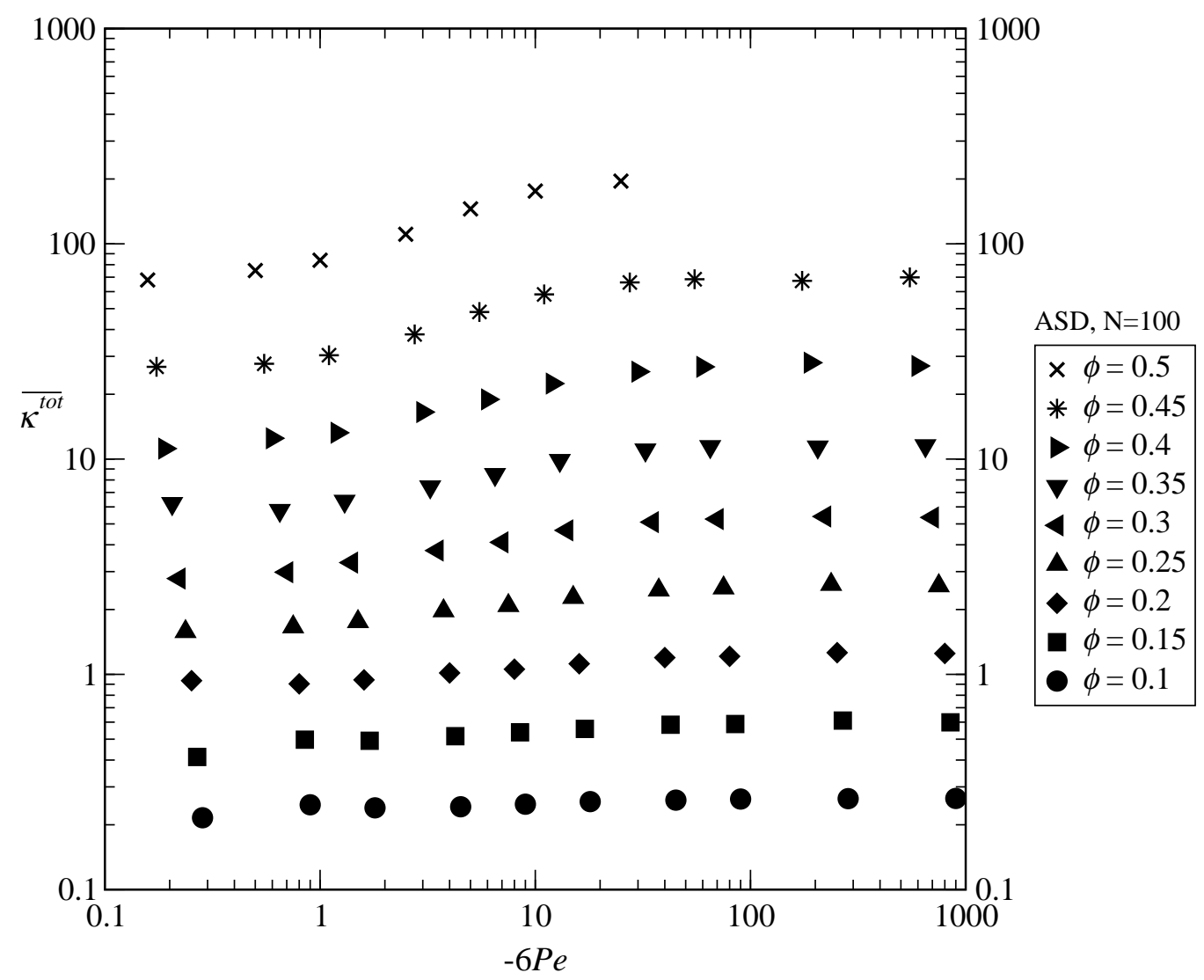

Figure 6.21: The total bulk viscosity contribution due to particle interactions $\overline{\kappa^{t o t}}=\left(\kappa^{H}+\kappa^{B}\right) / \eta$ vs Péclet number, from Accelerated Stokesian Dynamics - near field with $N=100$ for $\phi=0.1$ to $\phi=0.5$. 


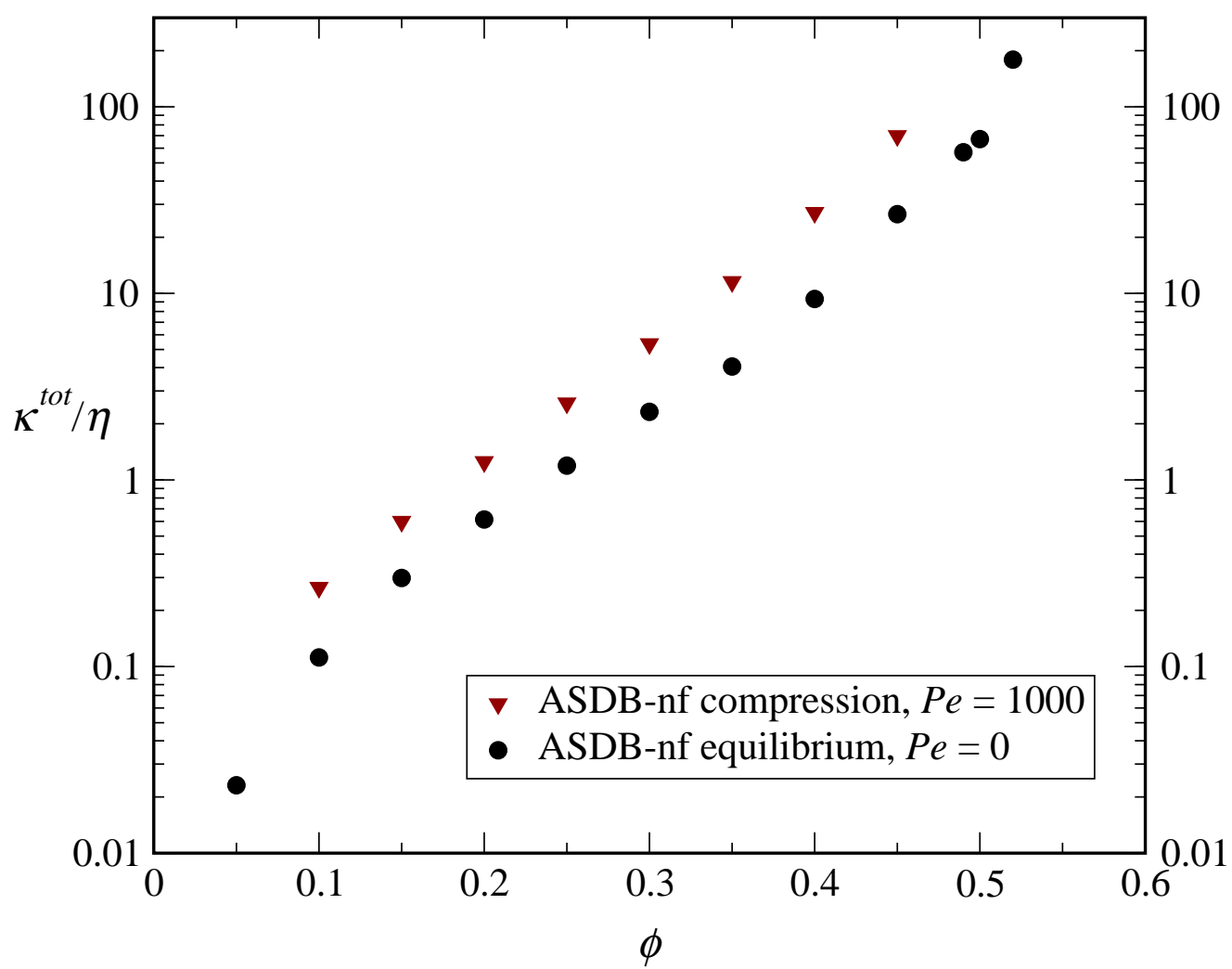

Figure 6.22: The total bulk viscosity at equilibrium and in compression with $P e=-1000$, vs the volume fraction $\phi$. In both cases the scaling with $\phi$ is the same and the difference in magnitude is not very significant. 


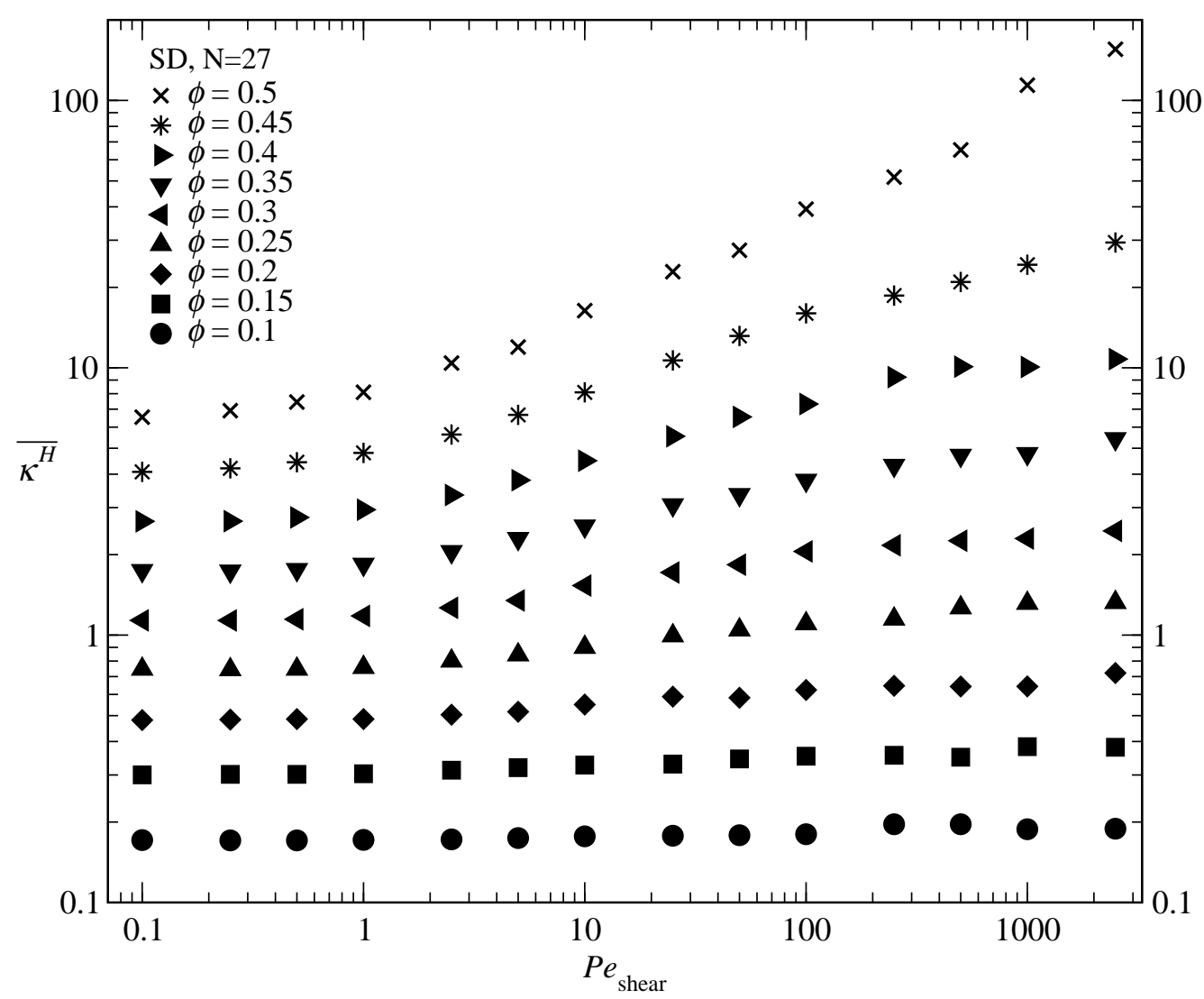

Figure 6.23: The hydrodynamic contribution to the bulk viscosity, also known as the high-frequency bulk viscosity $\left(\kappa_{\infty}^{\prime}\right)$, for a sheared suspension vs the Péclet number of shearing. 


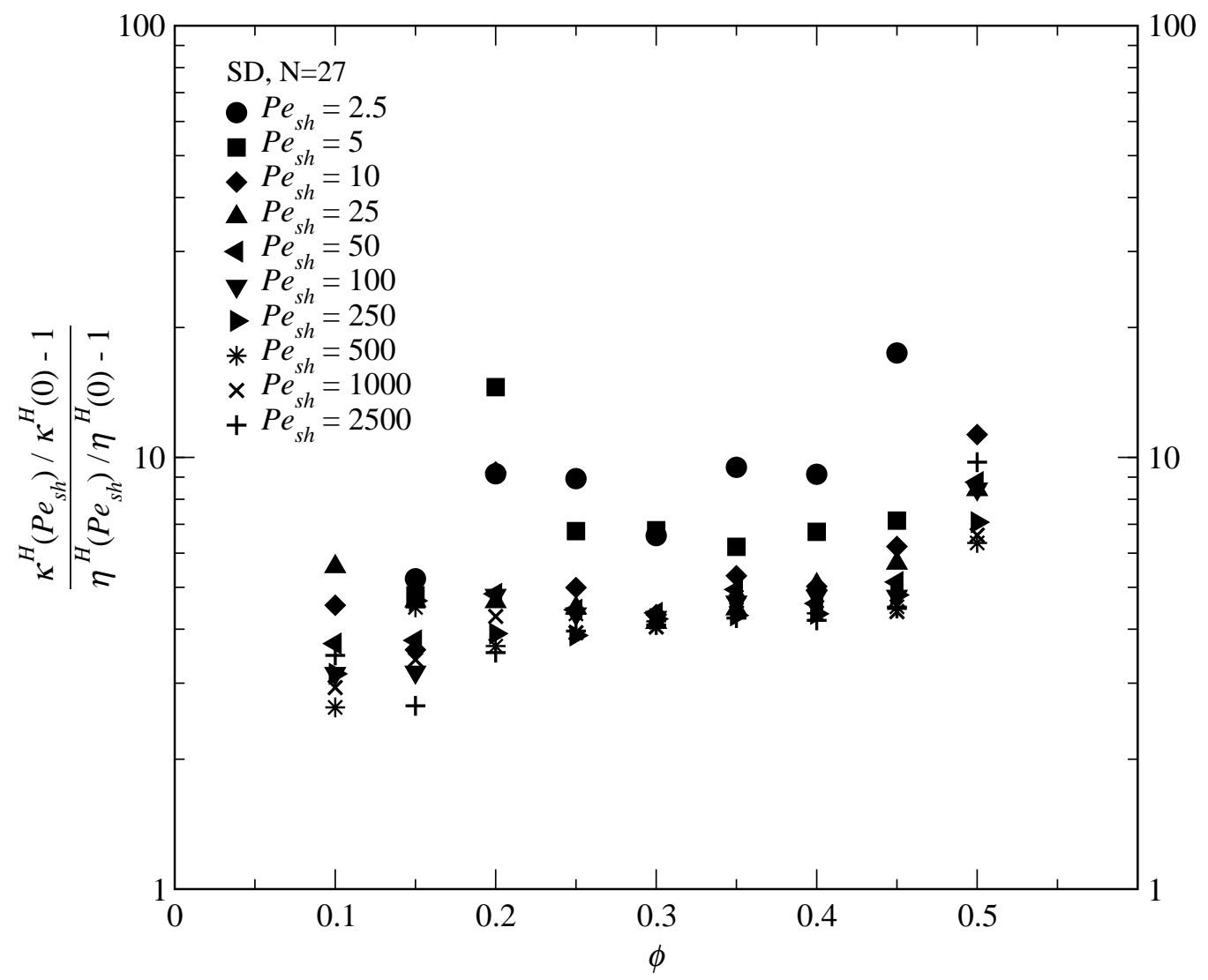

Figure 6.24: The effect of shearing on the high-frequency bulk viscosity $\left(\kappa_{\infty}^{\prime}\right)$ scaled with the effect of shearing on the high-frequency dynamic viscosity $\left(\eta_{\infty}^{\prime}\right)$ vs the volume fraction $\phi$. 


\begin{tabular}{|c|cccccccccc|}
\hline \multicolumn{10}{|c}{$\bar{\Pi}-1$} \\
\hline$-6 P e$ & 0.1 & 0.15 & 0.2 & 0.25 & 0.3 & 0.35 & 0.4 & 0.45 & 0.5 \\
\hline 0.1 & 1.5190 & 1.8825 & 2.3745 & 3.0096 & 3.8349 & 5.0084 & 6.5622 & 8.8082 & 12.229 \\
0.316 & 1.5495 & 1.9336 & 2.4432 & 3.0966 & 3.9718 & 5.1504 & 6.8050 & 9.2112 & 13.077 \\
1 & 1.6727 & 2.1598 & 2.7310 & 3.4846 & 4.5210 & 5.9998 & 7.9708 & 11.121 & 16.686 \\
3.16 & 1.9663 & 2.6066 & 3.4555 & 4.5756 & 6.0863 & 8.2350 & 11.445 & 16.717 & 26.842 \\
10 & 2.8392 & 4.1102 & 5.9026 & 8.2655 & 11.446 & 16.658 & 24.820 & 39.218 & 71.485 \\
31.6 & 5.4934 & 8.7891 & 13.433 & 19.521 & 29.008 & 44.074 & 68.728 & 115.88 & 224.09 \\
100 & 13.510 & 23.546 & 36.698 & 56.221 & 85.491 & 132.39 & 212.22 & 367.24 & 719.25 \\
316 & 39.122 & 69.455 & 109.39 & 170.97 & 262.28 & 408.79 & 660.44 & 1136.7 & 2272.9 \\
1000 & 122.22 & 215.08 & 349.63 & 538.59 & 836.87 & 1308.5 & 2104.6 & 3639.0 & 7317.7 \\
\hline
\end{tabular}

Table 6.1: The nondimensional particle-phase contribution to the osmotic pressure vs Pèclet number, from Brownian Dynamics simulations with $N=1000$, and 200 realizations each. 


\begin{tabular}{|c|c|c|c|c|c|c|c|c|c|}
\hline \multicolumn{10}{|c|}{$\operatorname{Error}( \pm)$ in $\bar{\Pi}-1$} \\
\hline & \multicolumn{9}{|c|}{$\phi$} \\
\hline$-6 P e$ & 0.1 & 0.15 & 0.2 & 0.25 & 0.3 & 0.35 & 0.4 & 0.45 & 0.5 \\
\hline 0.1 & 0.0640 & 0.0734 & 0.0983 & 0.1093 & 0.1291 & 0.1604 & 0.1828 & 0.2331 & 0.2963 \\
\hline 0.316 & 0.0904 & 0.1041 & 0.1225 & 0.1440 & 0.1691 & 0.2419 & 0.2388 & 0.3133 & 0.4207 \\
\hline 1 & 0.1260 & 0.1747 & 0.1980 & 0.2260 & 0.2866 & 0.3030 & 0.3810 & 0.4553 & 0.6292 \\
\hline 3.16 & 0.2245 & 0.2280 & 0.3029 & 0.4136 & 0.4699 & 0.5608 & 0.6969 & 0.9838 & 1.5487 \\
\hline 10 & 0.3833 & 0.5297 & 0.6686 & 0.7328 & 0.9156 & 1.2684 & 1.7294 & 2.6921 & 5.6285 \\
\hline 31.6 & 0.7985 & 1.1225 & 1.5682 & 1.7670 & 2.4100 & 3.1102 & 5.2710 & 8.7292 & 18.493 \\
\hline 100 & 1.8786 & 2.5282 & 3.7654 & 4.6476 & 7.3244 & 10.053 & 17.369 & 29.700 & 59.214 \\
\hline 316 & 4.6035 & 6.3652 & 9.4529 & 13.498 & 18.342 & 32.707 & 53.238 & 95.570 & 195.97 \\
\hline 1000 & 13.295 & 19.279 & 26.396 & 36.337 & 62.729 & 89.027 & 166.01 & 257.55 & 624.07 \\
\hline
\end{tabular}

Table 6.2: Error estimates $( \pm)$ for the nondimensional particle-phase contribution to the osmotic pressure vs Pèclet number, from Brownian Dynamics simulations with $N=1000$, and 200 realizations each. 


\begin{tabular}{|c|cccccccccc|}
\multicolumn{10}{c|}{$\kappa^{P} / \eta$} \\
\hline$-6 P e$ & 0.1 & 0.15 & 0.2 & 0.25 & 0.3 & 0.35 & 0.4 & 0.45 & 0.5 \\
\hline 0.316 & 0.0536 & 0.1010 & 0.1897 & 0.2552 & 0.3368 & 0.3812 & 0.5527 & 1.2203 & 4.9928 \\
1 & 0.0724 & 0.1846 & 0.3190 & 0.5171 & 0.8478 & 1.4583 & 2.2732 & 4.2526 & 9.6979 \\
3.16 & 0.0647 & 0.1539 & 0.3073 & 0.5521 & 0.9370 & 1.5755 & 2.6983 & 4.9318 & 10.300 \\
10 & 0.0597 & 0.1501 & 0.3173 & 0.5896 & 1.0196 & 1.8245 & 3.2601 & 6.1149 & 13.300 \\
31.6 & 0.0567 & 0.1475 & 0.3149 & 0.5873 & 1.0730 & 1.9438 & 3.5328 & 6.8477 & 15.075 \\
100 & 0.0540 & 0.1462 & 0.3089 & 0.5985 & 1.1016 & 2.0052 & 3.6992 & 7.2540 & 15.905 \\
316 & 0.0536 & 0.1443 & 0.3048 & 0.5979 & 1.1039 & 2.0122 & 3.7238 & 7.2263 & 16.096 \\
1000 & 0.0543 & 0.1439 & 0.3125 & 0.6025 & 1.1245 & 2.0528 & 3.7762 & 7.3507 & 16.437 \\
\hline
\end{tabular}

Table 6.3: The nondimensional hard sphere bulk viscosity vs Pèclet number, from Brownian Dynamics simulations with $N=1000$, and 200 realizations each. 


$$
\bar{\Pi}-1
$$

\begin{tabular}{|c|cccccccccc|}
\hline & \multicolumn{7}{|c|}{$\phi$} \\
\hline$\frac{-6 P e}{1-\phi}$ & 0.1 & 0.15 & 0.2 & 0.25 & 0.3 & 0.35 & 0.4 & 0.45 & 0.5 \\
\hline 0.1 & 0.5290 & 0.9329 & 1.4321 & 2.0816 & 3.0190 & 4.2455 & 6.3048 & 8.9834 & 13.298 \\
0.316 & 0.5402 & 0.9312 & 1.5010 & 2.1971 & 3.1552 & 4.6326 & 6.5801 & 9.9124 & 15.563 \\
1 & 0.6371 & 1.0768 & 1.6648 & 2.4705 & 3.5866 & 5.2696 & 8.1500 & 12.859 & 23.409 \\
2 & 0.7018 & 1.1982 & 1.9154 & 2.8652 & 4.2692 & 6.3803 & 10.073 & 17.011 & 33.957 \\
5 & 0.8825 & 1.5068 & 2.4311 & 3.7830 & 5.7415 & 9.1664 & 15.319 & 28.740 & 65.459 \\
10 & 1.0788 & 1.9096 & 3.0523 & 4.7490 & 7.4764 & 11.963 & 21.107 & 42.600 & 101.49 \\
20 & 1.2734 & 2.2221 & 3.6577 & 5.7830 & 9.4987 & 15.450 & 28.390 & 57.515 & 135.37 \\
50 & 1.4735 & 2.6452 & 4.3578 & 7.0357 & 11.407 & 18.875 & 35.000 & 71.747 & 155.18 \\
100 & 1.6512 & 2.9026 & 4.7584 & 7.6023 & 12.615 & 21.327 & 39.577 & 76.160 & - \\
316 & 1.7654 & 3.2593 & 4.9319 & 9.0381 & 13.110 & 24.251 & 42.647 & 70.700 & - \\
1000 & 1.9536 & 3.5005 & 5.8261 & 9.2456 & 15.625 & 30.478 & 43.324 & 66.090 & - \\
\hline
\end{tabular}

Table 6.4: The nondimensional particle-phase contribution to the osmotic pressure vs Pèclet number, from Accelerated Stokesian Dynamics - near field simulations with $N=100$, and 200 realizations each. 
$\operatorname{Error}( \pm)$ in $\bar{\Pi}-1$

\begin{tabular}{|c|c|c|c|c|c|c|c|c|c|}
\hline & \multicolumn{9}{|c|}{$\phi$} \\
\hline$\frac{-6 P e}{1-\phi}$ & 0.1 & 0.15 & 0.2 & 0.25 & 0.3 & 0.35 & 0.4 & 0.45 & 0.5 \\
\hline 0.1 & 0.0726 & 0.1152 & 0.1305 & 0.1863 & 0.2147 & 0.2646 & 0.4797 & 0.5111 & 0.7999 \\
\hline 0.316 & 0.0871 & 0.1309 & 0.1786 & 0.2179 & 0.2710 & 0.3930 & 0.4507 & 0.7665 & 1.1174 \\
\hline 1 & 0.1221 & 0.1966 & 0.1991 & 0.2987 & 0.4063 & 0.5924 & 0.8019 & 1.5166 & 3.6437 \\
\hline 2 & 0.1333 & 0.1998 & 0.2606 & 0.3645 & 0.4900 & 0.7947 & 1.2031 & 2.3982 & 6.0027 \\
\hline 5 & 0.1970 & 0.2293 & 0.4425 & 0.6050 & 0.8006 & 1.4047 & 2.3825 & 4.6437 & 13.445 \\
\hline 10 & 0.2201 & 0.3421 & 0.5330 & 0.6950 & 1.0683 & 2.0306 & 3.7724 & 9.6432 & 25.685 \\
\hline 20 & 0.2652 & 0.3653 & 0.5927 & 0.9430 & 1.5286 & 2.6536 & 5.3461 & 12.514 & 31.319 \\
\hline 50 & 0.3162 & 0.5213 & 0.8386 & 1.0913 & 2.1729 & 4.2318 & 8.6484 & 18.868 & 45.224 \\
\hline 100 & 0.3715 & 0.5175 & 0.8278 & 1.4961 & 3.7415 & 7.9296 & 17.399 & 40.538 & - \\
\hline 316 & 1.1666 & 0.6669 & 2.7372 & 5.9231 & 12.847 & 23.049 & 51.255 & 114.57 & - \\
\hline 1000 & 0.5193 & 0.7424 & 4.6460 & 7.4982 & 40.662 & 64.268 & 142.66 & 224.71 & - \\
\hline
\end{tabular}

Table 6.5: Error estimates $( \pm)$ for the nondimensional particle-phase contribution to the osmotic pressure vs Pèclet number, from Accelerated Stokesian Dynamics - near field simulations with $N=100$, and 200 realizations each. 


\section{$\kappa^{B} / \eta$}

\begin{tabular}{|c|ccccccccc|}
\hline & \multicolumn{1}{|c|}{$\phi$} \\
\hline$\frac{-6 P e}{1-\phi}$ & 0.1 & 0.15 & 0.2 & 0.25 & 0.3 & 0.35 & 0.4 & 0.45 & 0.5 \\
\hline 0.1 & 0.0388 & 0.2318 & 0.2908 & 0.1127 & 0.8715 & 0.9632 & 11.366 & 22.043 & 58.430 \\
0.316 & 0.0300 & 0.0691 & 0.3375 & 0.5839 & 1.1075 & 3.2734 & 6.2109 & 17.800 & 50.740 \\
1 & 0.0579 & 0.1374 & 0.2909 & 0.5946 & 1.1820 & 2.5777 & 6.6723 & 16.474 & 51.342 \\
2 & 0.0451 & 0.1169 & 0.2864 & 0.5933 & 1.2492 & 2.6346 & 6.2201 & 15.881 & 49.403 \\
5 & 0.0361 & 0.0958 & 0.2306 & 0.5127 & 1.0676 & 2.4040 & 5.6358 & 14.989 & 48.113 \\
10 & 0.0279 & 0.0799 & 0.1852 & 0.4012 & 0.8684 & 1.8795 & 4.5544 & 12.598 & 40.270 \\
20 & 0.0188 & 0.0524 & 0.1266 & 0.2782 & 0.6292 & 1.3622 & 3.3696 & 9.0444 & 27.759 \\
50 & 0.0095 & 0.0277 & 0.0664 & 0.1489 & 0.3253 & 0.7109 & 1.7444 & 4.6658 & 12.886 \\
100 & 0.0057 & 0.0159 & 0.0377 & 0.0829 & 0.1859 & 0.4149 & 1.0095 & 2.4954 & - \\
316 & 0.0020 & 0.0059 & 0.0126 & 0.0331 & 0.0619 & 0.1537 & 0.3486 & 0.7261 & - \\
1000 & 0.0007 & 0.0021 & 0.0050 & 0.0108 & 0.0244 & 0.0637 & 0.1122 & 0.2125 & - \\
\hline
\end{tabular}

Table 6.6: The nondimensional Brownian contribution to the bulk viscosity with hydrodynamic interactions vs Pèclet number, from Accelerated Stokesian Dynamics — near field simulations with $N=100$, and 200 realizations each. 
$\overline{k^{H}}$

\begin{tabular}{|c|cccccccccc|}
\hline & \multicolumn{7}{|c|}{$\phi$} \\
\hline$\frac{-6 P e}{1-\phi}$ & 0.1 & 0.15 & 0.2 & 0.25 & 0.3 & 0.35 & 0.4 & 0.45 & 0.5 \\
\hline 0.1 & 0.1663 & 0.2926 & 0.4691 & 0.7318 & 1.1331 & 1.7563 & 2.8806 & 4.5507 & 7.4523 \\
0.316 & 0.1672 & 0.2924 & 0.4780 & 0.7467 & 1.1744 & 1.9128 & 2.9877 & 4.9679 & 8.5945 \\
1 & 0.1717 & 0.3054 & 0.4864 & 0.7998 & 1.2580 & 2.0848 & 3.5189 & 6.1647 & 12.095 \\
2 & 0.1751 & 0.3182 & 0.5251 & 0.8732 & 1.4376 & 2.4287 & 4.2219 & 7.9705 & 17.246 \\
5 & 0.1858 & 0.3570 & 0.6274 & 1.0944 & 1.8855 & 3.2687 & 6.5384 & 12.630 & 31.305 \\
10 & 0.1992 & 0.3893 & 0.6971 & 1.2635 & 2.2655 & 4.2893 & 8.6275 & 19.602 & 52.535 \\
20 & 0.2137 & 0.4297 & 0.7946 & 1.4986 & 2.8244 & 5.5235 & 11.438 & 27.036 & 74.026 \\
50 & 0.2257 & 0.4723 & 0.9037 & 1.7360 & 3.3402 & 6.7014 & 14.226 & 33.811 & 91.306 \\
100 & 0.2319 & 0.4859 & 0.9393 & 1.8275 & 3.5571 & 7.1634 & 15.487 & 36.276 & - \\
316 & 0.2362 & 0.5134 & 0.9983 & 1.9375 & 3.7446 & 7.2962 & 16.609 & 36.611 & - \\
1000 & 0.2379 & 0.5071 & 0.9961 & 1.9268 & 3.7321 & 7.4580 & 16.174 & 38.315 & - \\
\hline
\end{tabular}

Table 6.7: The nondimensional Hydrodynamic bulk viscosity contribution $\overline{\kappa^{H}}=\kappa^{H} / \eta$ vs Pèclet number, from Accelerated Stokesian Dynamics - near field simulations with $N=100$, and 200 realizations each. 
Error $( \pm)$ in $\overline{\kappa^{H}}$

\begin{tabular}{|c|ccccccccc|}
\hline & \multicolumn{10}{|c|}{$\phi$} \\
\hline$\frac{-6 P e}{1-\phi}$ & 0.1 & 0.15 & 0.2 & 0.25 & 0.3 & 0.35 & 0.4 & 0.45 & 0.5 \\
\hline 0.1 & 0.0056 & 0.0160 & 0.0225 & 0.0532 & 0.0715 & 0.0982 & 0.2061 & 0.2347 & 0.4051 \\
0.316 & 0.0107 & 0.0162 & 0.0323 & 0.0522 & 0.0852 & 0.1730 & 0.2084 & 0.3627 & 0.5699 \\
1 & 0.0060 & 0.0215 & 0.0268 & 0.0633 & 0.1039 & 0.1991 & 0.2978 & 0.6584 & 1.7395 \\
2 & 0.0073 & 0.0198 & 0.0338 & 0.0706 & 0.1388 & 0.2487 & 0.4429 & 1.0337 & 2.8281 \\
5 & 0.0088 & 0.0271 & 0.0612 & 0.1244 & 0.2148 & 0.4117 & 1.0312 & 1.8455 & 5.9865 \\
10 & 0.0111 & 0.0308 & 0.0583 & 0.1236 & 0.2538 & 0.6444 & 1.4966 & 4.1887 & 13.903 \\
20 & 0.0137 & 0.0369 & 0.0782 & 0.1779 & 0.3851 & 0.8743 & 2.0010 & 6.1463 & 17.812 \\
50 & 0.0168 & 0.0449 & 0.0998 & 0.2443 & 0.5373 & 1.2559 & 2.7754 & 7.3935 & 26.927 \\
100 & 0.0188 & 0.0494 & 0.1098 & 0.2391 & 0.5117 & 1.2352 & 3.0167 & 7.8312 & - \\
316 & 0.0195 & 0.0533 & 0.1235 & 0.2512 & 0.5573 & 1.1766 & 3.3583 & 7.8972 & - \\
1000 & 0.0181 & 0.0486 & 0.1162 & 0.2414 & 0.5524 & 1.3172 & 2.8793 & 7.8422 & - \\
\hline
\end{tabular}

Table 6.8: Error estimates $( \pm)$ for the nondimensional Hydrodynamic bulk viscosity contribution $\overline{\kappa^{H}}=\kappa^{H} / \eta$ vs Pèclet number, from Accelerated Stokesian Dynamics — near field simulations with $N=100$, and 200 realizations each. 


\section{Chapter 7}

\section{Concluding Remarks}

We have defined the effective bulk viscosity for a suspension of particles in a fluid in a way analogous to the definition of bulk viscosity for a pure fluid. The bulk viscosity relates the deviation of the trace of the macroscopic stress from its equilibrium value to the average rate of expansion of the suspension. Previously the effective dynamic viscosity which gives the rheological response in shear flow, and the microviscosity which gives the resistance experienced by a probe as it travels through a suspension have been studied in detail. The bulk viscosity is the third kind of suspension viscosity and thus completes the set of rheological problems that can be studied. Indeed the monopolar nature of the forcing in the linear response regime sets the bulk viscosity apart from the other two viscosities in that it has the slowest spatial and temporal response.

Expressions were derived for computing the bulk viscosity of a suspension undergoing uniform expansion for all volume fractions of particles and for all expansion rates. The Stokesian Dynamics and Accelerated Stokesian Dynamics techniques for simulation of suspensions in Stokes flow were modified to include expansion and compression of the fluid and the particle phase. The updated simulation techniques can now be used to explore new kinds of flows such as particles that dynamically change size. Our simulation results show that the bulk viscosity diverges at maximum packing just like the shear viscosity and both are comparable in magnitude. At high rates of compression there is enhancement of the bulk viscosity similar to the 'thickening' effect in a sheared suspension but the high $P e$ plateau for the bulk viscosity is smaller than the shear viscosity. Care must be taken in defining the bulk viscosity at very high rates of compression. If the time scale for the evolution of the microstructure is the same as that for the number density, which will be the case at large Péclet number, then a steady bulk viscosity will not exist. In such cases the bulk viscosity can be 
estimated as being higher than its equilibrium value but smaller than its value in the large $P e$ limit. If the suspension is undergoing uniform expansion, initially when the microstructure is undisturbed the bulk viscosity would be given by its equilibrium value but as the particles move away from each other the stress due to particle interactions will decrease rapidly and eventually the bulk viscosity would be negligible.

In this work we have determined the bulk viscosity by applying a uniform rate of expansion to the suspending fluid, which drives the expansion of the particle phase. However all the subsequent interactions between particles are due to incompressible disturbance flows and would be present as long as the particles are moving away from each other, even if the fluid is incompressible. Of course in such a flow there would be an additional contribution due to the drag force of the fluid as it squeezes between the particles, and this can be added separately from previously known expressions. For example, consider a suspension between two filters such that the fluid can pass through the filter material but the particles cannot (see Figure 7.1). If the filters are pressed together the fluid will have to squeeze through the particle phase and come out of the filter. The particle phase on the other hand will undergo a compression on the macroscopic scale as the particles are pushed closer. The contribution to the stress due to hydrodynamic interactions between the particles as they move is the piece given by the effective suspension bulk viscosity.

To appreciate the effects of the bulk viscosity on suspension transport, consider a 1D flow problem like the squeezing flow above. The standard mass and momentum balances for he particle phase take the form

$$
\begin{gathered}
\frac{\partial \phi}{\partial t}+\frac{\partial}{\partial z} \phi u_{z}^{p}=0 \\
0=-\frac{9}{2} \eta a^{-2} \phi R(\phi) u_{z}^{p}+\frac{\partial \Sigma_{z z}^{p}}{\partial z},
\end{gathered}
$$

where $R(\phi)$ is the drag coefficient for the hydrodynamic drag force exerted by the fluid on the particles.

In the absence of any bulk viscosity effect, (7.2) can be solved for the $z$-component of the particle phase velocity and substituted into the mass balance to give a diffusion equation for the particle concentration

$$
\frac{\partial \phi}{\partial t}=\frac{\partial}{\partial z}\left(\frac{2}{9} \frac{a^{2}}{\eta R}\right) \frac{\partial \Pi}{\partial z}
$$


This is the standard form of the shear-induced migration or diffusive flux model that has been used to model suspension flows [Phillips et al. 1992; Leighton and Acrivos 1987b]. (Note that the osmotic pressure in viscous suspensions is proportional to the shear rate.) The above simple derivation [Nott and Brady 1994] shows that one should more properly consider stress-induced diffusion or migration. The bulk viscosity changes the dynamics because (7.2) now becomes a differential equation for $u_{z}^{p}$ and the temporal evolution of the concentration profile will be different. Note that at steady state $u_{z}^{p}=0$, there is a simple mechanical balance of stresses and the effects of the bulk viscosity go away, as they should. Whenever we have unsteady flows, especially when there are rapid spatial variations in concentration (which would give a large $\nabla \cdot \boldsymbol{u}_{p}$ ), bulk viscosity effects will influence the dynamics.

One should also be able to measure the bulk viscosity experimentally. The result obtained here, that the change in bulk viscosity due to the presence of particles is proportional to the shear viscosity of the fluid, may be used to design appropriate experiments. A direct way to measure the bulk viscosity would be to suspend the particles in a gas (say in a microgravity environment to reduce sedimentation) and then expand or compress the gas and measure the pressure required to do so. Apart from this direct approach one can use macroscopic models of suspension behavior to infer the bulk viscosity. The idea here is to add to the particle-phase momentum balance, a bulk viscosity term proportional to the divergence of the particle phase velocity, $\kappa \boldsymbol{\nabla} \cdot \boldsymbol{u}_{p}$. Then when compressing the particle phase, in filtration for example, there will be a stress generated proportional to the rate of compression. Comparison of the experimental compression curve with the model predictions will allow one to back out the bulk viscosity. It should be noted that there are certain compression/expansion flows that cannot be properly modeled without acknowledging bulk viscosity effects. For example, the transient expansion to fluidized beds at low Reynolds number requires a stress contribution proportional to the rate of expansion. 


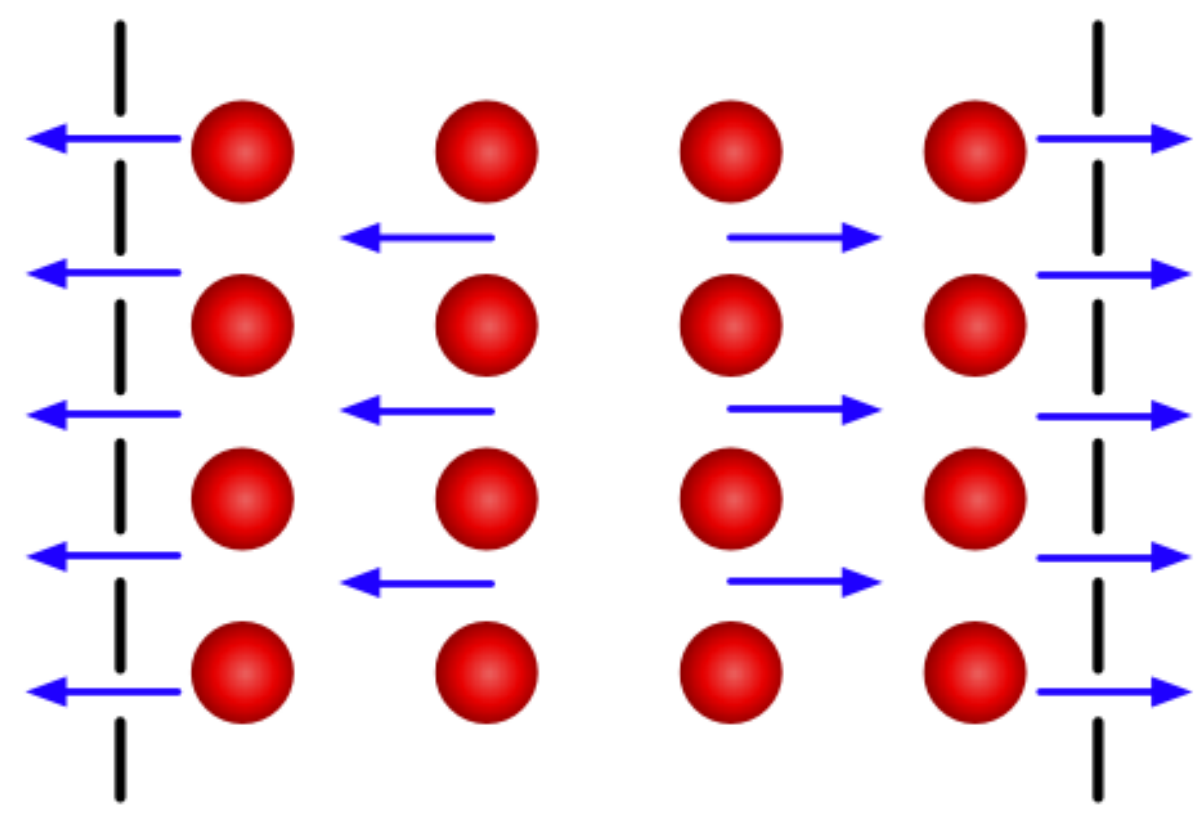

Figure 7.1: The suspending fluid is squeezed out as the filters enclosing a suspension are pushed closer causing compression of the particle phase as they are pushed closer to each other. 


\section{Bibliography}

Abramowitz, M. and I. A. Stegun, Handbook of Mathematical Functions with Formulas, Graphs, and Mathematical Tables (Dover Publications, New York, 1964).

Banchio, A. J. and J. F. Brady, "Accelerated Stokesian Dynamics - Brownian motion,” J. Chem. Phys. 118 (22), 10323-10332 (2003).

Banchio, A. J., G. Nagèle, and J. Bergenholtz, "Viscoelasticity and generalized Stokes - Einstein relations of colloidal dispersions," J. Chem. Phys. 111 (18), 8721-8740 (1999).

Batchelor, G. K., "The stress system in a suspension of force-free particles," J. Fluid Mech. 41, $545-570$ (1970).

Batchelor, G. K., "Transport properties of two-phase materials with random structure," Annu. Rev. Fluid Mech. 6, 227-255 (1974).

Batchelor, G. K., "Brownian diffusion of particles with hydrodynamic interaction," J. Fluid Mech. 74, 1-29 (1976).

Batchelor, G. K., "The effect of Brownian motion on the bulk stress in a suspension of spherical particles,” J. Fluid Mech. 83, 97-117 (1977).

Batchelor, G. K. and J. T. Green, “The hydrodynamic interaction of two small freely-moving spheres in a linear flow field,” J. Fluid Mech. 56, 375-400 (1972a).

Batchelor, G. K. and J. T. Green, "The determination of the bulk stress in a suspension of spherical particles to order $c^{2}$," J. Fluid Mech. 56, 401-427 (1972b).

Beenakker, C. W. J., "Ewald sum of the Rotne-Prager tensor," Journal of Chemical Physics 85 (3), 1581-1582 (1986). 
Bergenholtz, J., J. F. Brady, and M. Vicic, “The non-Newtonian rheology of dilute colloidal suspensions,” J. Fluid Mech. 456, 239-275 (2002).

Brady, J. F., "Self-Diffusion of Brownian particles in concentrated suspensions under shear," J. Chem. Phys. 87 (9), 5437-5448 (1987).

Brady, J. F., "Brownian motion, hydrodynamics, and the osmotic pressure," J. Chem. Phys. 98, 3335-3341 (1993a).

Brady, J. F., "The rheological behavior of concentrated colloidal dispersions," J. Chem. Phys. 99 (1), 567-581 (1993b).

Brady, J. F., "The long-time self-diffusivity in concentrated colloidal dispersions," J. Fluid Mech. 272, $109-133$ (1994).

Brady, J. F. and G. Bossis, "Stokesian dynamics," Annu. Rev. Fluid Mech. 20, 111-157 (1988).

Brady, J. F. and G. Bossis, "The rheology of Brownian suspensions,” J. Chem. Phys. 91 (3), 18661874 (1989).

Brady, J. F. and D. R. Foss, "Structure, diffusion and rheology of Brownian suspensions by Stokesian Dynamics simulation,” J. Fluid Mech. 407, 167-200 (2000).

Brady, J. F., A. S. Khair, and M. Swaroop, "On the bulk viscosity of suspensions," J. Fluid Mech. 554, 109-123 (2006).

Brady, J. F. and J. F. Morris, "Microstructure of strongly sheared suspensions and its impact on rheology and diffusion,” J. Fluid Mech. 348, 103-139 (1997).

Carnahan, N. F. and K. E. Starling, "Equation of State for Nonattracting Rigid Spheres," J. Chem. Phys. 51 (2), 635-636 (1969).

Cheng, Z., J. Zhu, P. M. Chaikin, S.-E. Phan, and W. B. Russel, "Nature of the divergence in low shear viscosity of colloidal hard-sphere dispersions," Physical Review E 65, 041405 (2002).

Cichocki, B. and B. U. Felderhof, "Linear viscoelasticity of semidilute hard-sphere suspensions," Physical Review A 43 (10), 5405 - 5411 (1991). 
Durlofsky, L. J., J. F. Brady, and G. Bossis, "Dynamic simulation of hydrodynamically interacting particles," J. Fluid Mech. 180, 21-49 (1987).

Eu, B. C., Nonequilibrium Statistical Mechanics (Springer, 1998).

Fang, Z., A. A. Mammoli, J. F. Brady, M. S. Ingber, L. A. Mondy, and A. L. Graham, "Flow-aligned tensor models for suspension flows," Int. J. Multiphase Flow 28, 137-166 (2002).

Foss, D. R., Rheological Behavior of Colloidal Suspensions: the Effects of Hydrodynamic Interactions, PhD thesis California Institute of Technology 1999.

Foss, D. R. and J. F. Brady, "Self-diffusion in sheared suspensions by dynamic simulation," J. Fluid Mech. 401, 243-274 (1999).

Foss, D. R. and J. F. Brady, "Brownian Dynamics simulation of hard-sphere colloidal dispersions," Journal of Rheology 44 (3), 629-651 (2000).

Gadala-Maria, F. and A. Acrivos, "Shear-induced structure in a concentrated suspension of solid spheres," J. Rheol. 24 (6), 799 (1980).

Gidaspow, D., Multiphase flow and fluidization (Academic Press, 1994).

Green, H. S., The Molecular Theory of Fluids (North-Holland, 1952).

Hasimoto, H., "On the periodic fundamental solutions of the Stokes equations and their application to viscous flow past a cubic array of apheres," J. Fluid Mech. 5, 317 - 328 (1959).

Hockney, R. W. and J. W. Eastwood, Computer simulation using particles (I. O. P. Publishing, Philadelphia, 1988).

Hollenbeck, K. J., "INVLAP.M: A matlab function for numerical inversion of Laplace transforms by the de Hoog algorithm," 1998, URL http://www . mathworks . com/matlabcentral/linkexchange/links/74-invlapm.

Hoover, W. G., A. J. C. Ladd, and R. B. Hickam, "Bulk viscosity via nonequilibrium and equilibrium molecular dynamics," Physical Review A 21 (5), 1756-1760 (1980). 
Jeffrey, D. J., J. F. Morris, and J. F. Brady, “The pressure moments for two rigid spheres in lowReynolds-number flow," Phys. Fluids A 5, 2317-2325 (1993).

Jeffrey, D. J. and Y. Onishi, "Calculation of the resistance and mobility functions for two unequal rigid spheres in low-Reynolds-number-flow,” J. Fluid Mech. 139, 261-290 (1984).

Keizer, J., Statistical Thermodynamics of Nonequilibrium Processes (Springer, 1987).

Khair, A. S. and J. F. Brady, ““Microviscoelasticity” of colloidal dispersions,” Journal of Rheology 49 (6), 1449-1481 (2005).

Khair, A. S. and J. F. Brady, "Single particle motion in colloidal dispersions: a simple model for active and nonlinear microrheology," J. Fluid Mech. 557, 73-117 (2006).

Khair, A. S., M. Swaroop, and J. F. Brady, "A new resistance function for two rigid spheres in low-Reynolds number flow," Phys. Fluids 18, 043102 (2006).

Kim, S. and S. J. Karrila, Microhydrodynamics: Principles and Selected Applications (ButterworthHeinemann, Boston, 1991).

Kirkwood, J. G., F. P. Buff, and M. S. Green, "The Statistical Mechanical Theory of Transport Processes. III. The Coefficients of Shear and Bulk Viscosity of Liquids," J. Chem. Phys. 17 (10), 988-994 (1949).

Ladd, A. J. C., "Hydrodynamic transport coefficients of random dispersions of hard spheres," J. Chem. Phys. 93, 3484-3494 (1990).

Lebedev, N. N., Special Functions and their Applications (Dover Publications, New York, 1972).

Leighton, D. and A. Acrivos, "The shear-induced migration of particles in concentrated suspensions," J. Fluid Mech. 181, 415-439 (1987b).

Lionberger, R. A. and W. B. Russel, "High frequency modulus of hard sphere colloids," Journal of Rheology 38, 1885-1908 (1994).

Lun, C. K. K., S. B. Savage, D. J. Jeffrey, and N. Chepurniy, "Kinetic theories of granular flow: inelastic particles in Couette flow and slightly inelastic particles in a general flow field,' J. Fluid Mech. 140, 223-256 (1984). 
Malbrunot, P., A. Boyer, and E. Charles, "Experimental bulk viscosities of argon, krypton, and xenon near their triple point," Physical Review A 27 (3), 1523-1534 (1983).

Nagèle, G. and J. Bergenholtz, "Linear viscoelasticity of colloidal mixtures," J. Chem. Phys. 108 (23), 9893-9904 (1998).

Nott, P. R. and J. F. Brady, "Pressure-driven flow of suspensions: simulation and theory," J. Fluid Mech. 275, 157-199 (1994).

Phillips, R. J., R. C. Armstrong, R. A. Brown, A. Graham, and J. R. Abbott, “A constitutive model for concentrated suspensions that accounts for shear-induced particle migration," Phys. Fluids A 4, 30-40 (1992).

Phillips, R. J., J. F. Brady, and G. Bossis, "Hydrodynamic transport properties of hard-sphere dispersions. I. Suspensions of freely mobile particles," Phys. Fluids 31, 3462-3472 (1988).

Phung, T. N., Behavior of Concentrated Colloidal Suspensions by Stokesian Dynamics Simulation, PhD thesis California Institute of Technology 1992.

Rah, K. and B. C. Eu, “Analog of the Stokes-Einstein Relation for Bulk Viscosity,” Physical Review Letters 83 (22), 4566-4569 (1999).

Rintoul, M. D. and S. Torquato, "Metastability and Crystallization in Hard-Sphere Systems," Physical Review Letters 77 (20), 4198 - 4201 (1996).

Russel, W. B., D. A. Saville, and W. R. Schowalter, Colloidal Dispersions (Cambridge University Press, Cambridge, UK, 1989).

Schiesser, W. E., The numerical method of lines: integration of partial differential equations (Academic Press, 1991).

Segrè, P. N., S. P. Meeker, P. N. Pusey, and W. C. K. Poon, "Viscosity and Structural Relaxation in Suspensions of Hard-Sphere Colloids,” Phys. Rev. Lett. 75 (5), 958-961 (1995).

Shampine, L. F. and M. W. Reichelt, “The MATLAB ODE Suite," SIAM Journal on Scientific Computing 18, 1-22 (1997). 
Sierou, A., Accelerated Stokesian Dynamics: Development and application to sheared nonBrownian suspensions, $\mathrm{PhD}$ thesis California Institute of Technology 2002.

Sierou, A. and J. F. Brady, “Accelerated Stokesian Dynamics simulations," J. Fluid Mech. 448, $115-146(2001)$.

Sierou, A. and J. F. Brady, "Rheology and microstructure in concentrated noncolloidal suspensions," J. Rheol. 46, 1031-1056 (2002).

Sigurgeirsson, H. and D. M. Heyes, "Transport coefficients of hard sphere fluids," Mo. Phys. 101, 469-482 (2003).

Skoge, M., A. Donev, F. H. Stillinger, and S. Torquato, "Packing Hyperspheres in High-Dimensional Euclidean Spaces,” Physical Review E 74, 041127 (2006).

Speedy, R. J., “On the reproducibility of glasses,” J. Chem. Phys. 100 (9), 6684-6691 (1994).

Swaroop, M. and J. F. Brady, "The bulk viscosity of suspensions," J. Rheol. 51 (3), 409-428 (2007).

Taylor, G. I., "The two coefficients of viscosity for an incompressible fluid containing air bubbles," Proc. R. Soc. Lond. A 226, 34-37 (1954a).

Taylor, G. I., "Note on the volume viscosity of water containing bubbles," Proc. R. Soc. Lond. A 226, 38-39 (1954b).

Werff, J. C.van der, C. G. de Kruif, C. Blom, and J. Mellema, "Linear viscoelastic behavior of dense hard-sphere dispersions," Physical Review A 39 (2), 795-807 (1989).

Zwanzig, R. and R. D. Mountain, "High-Frequency Elastic Moduli of Simple Fluids,” J. Chem. Phys. 43 (12), 4464-4471 (1965). 